\title{
Rhodium-Catalyzed 1,1-Hydroacylation of Thioacyl Carbenes with Alkynyl Aldehydes and Subsequent Cyclization
}

Bingnan Zhou, Qiuyue Wu, Ziyang Dong, Jiaxi Xu* and Zhanhui Yang*

State Key Laboratory of Chemical Resource Engineering, Department of Organic Chemistry, Faculty of Science, Beijing University of Chemical Technology, Beijing 100029, P. R. China

\section{Corresponding Author}

* zhyang@mail.buct.edu.cn (Z.Y.)

*jxxu@mail.buct.edu.cn (J.X.) 


\section{Contents}

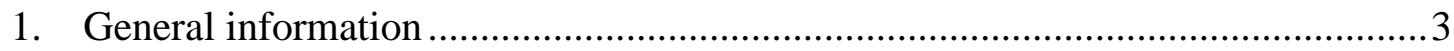

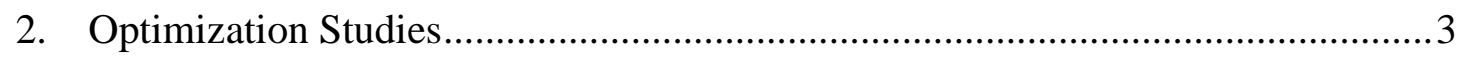

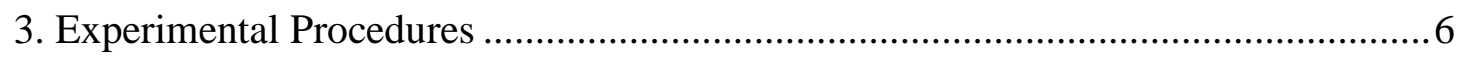

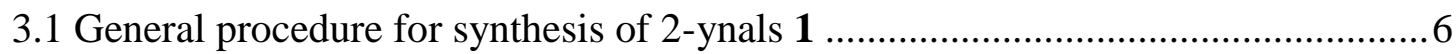

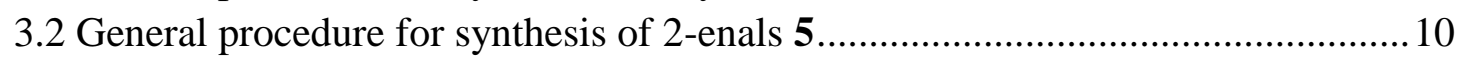

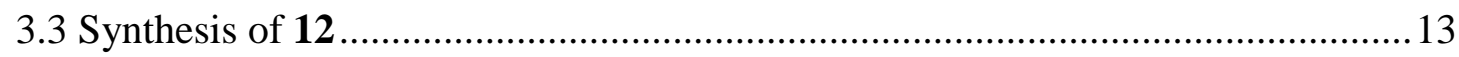

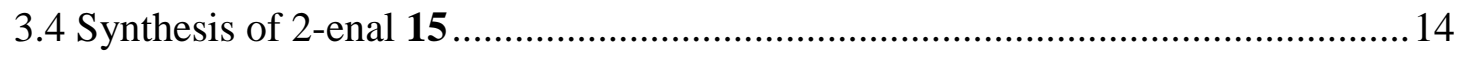

4. Experimental procedure for the reactions of thiodiazoles 2 and ynals 1..............15

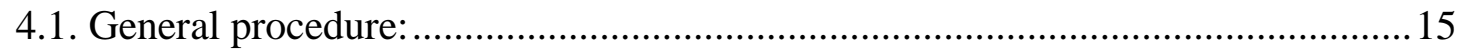

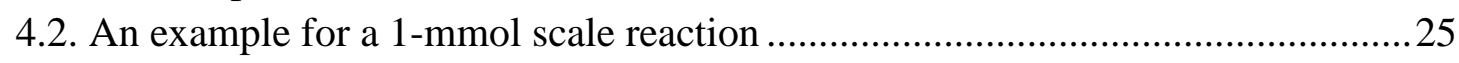

5. Experimental procedure for the reactions of thiodiazoles 2 and enals 4 ............25

6. Oxidation of 4aa and its X-Ray Crystallographic Analysis .............................29

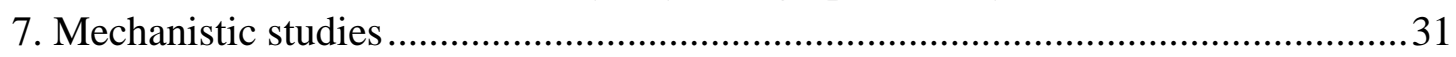

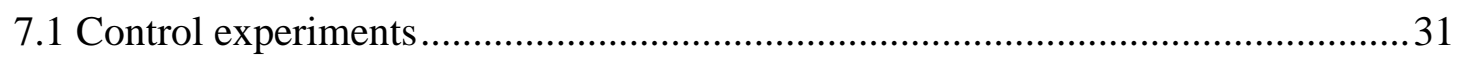

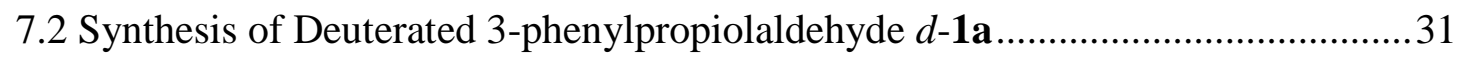

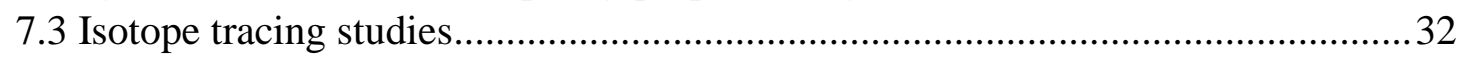

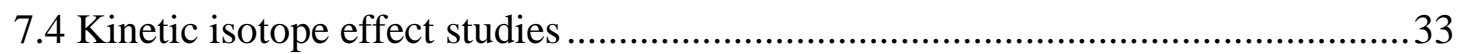

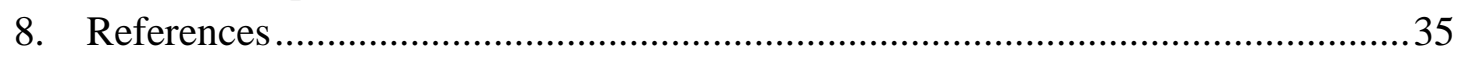

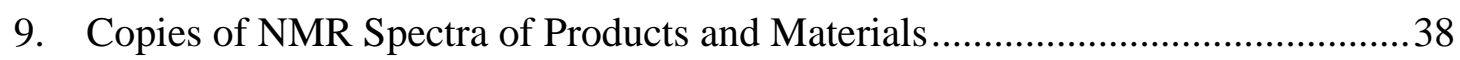




\section{General information}

Unless otherwise noted, all reactions were performed in ordinary reaction tubes. All materials were purchased from commercial suppliers. Dichloromethane (DCM) and chlorobenzene were refluxed over $\mathrm{CaH}_{2}$; tetrahydrofuran (THF) was refluxed over lithium aluminum hydride. The solvents were freshly distilled prior to use. Column chromatography was performed using silica gel (normal phase, 200-300 mesh) from Branch of Qingdao Haiyang Chemical, with petroleum ether (PE, bp. 60-90 ${ }^{\circ} \mathrm{C}$ ) and ethyl acetate (EA) as eluent. Reactions were monitored by thin-layer chromatography on GF254 silica gel plates $(0.2 \mathrm{~mm})$ from Yantai Athy Chemical Technology Industry. The plates were visualized by UV light, and other TLC stains (10\% phosphomolybdic acid in ethanol; $1 \%$ potassium permanganate in water; $10 \mathrm{~g}$ of iodine absorbed on $30 \mathrm{~g}$ of silica gel). ${ }^{1} \mathrm{H}$ and ${ }^{13} \mathrm{C}$ NMR spectra were recorded on a Bruker $400 \mathrm{MHz}$ spectrometer, usually in $\mathrm{CDCl}_{3}$ as an internal standard, and the chemical shifts $(\delta)$ were reported in parts per million ( $\mathrm{ppm})$. Multiplicities are indicated as s (singlet), $\mathrm{d}$ (doublet), $\mathrm{t}$ (triplet), q (quartet), dd (double doublet), m (multiplet), and b (broad). Coupling constants $(J)$ are reported in Hertz $(\mathrm{Hz})$. The IR spectra (film on $\mathrm{KBr}$ pellets, $v\left[\mathrm{~cm}^{-1}\right]$ ) were taken on a Nicolet 5700 FTIR spectrometer. HRMS measurements were carried out on an Agilent LC/MSD TOF mass spectrometer. Melting points were obtained on a Yanaco MP-500 melting point apparatus and are uncorrected.

Aldehydes 1a, $\mathbf{5 a}, \mathbf{5 c}, \mathbf{5 k}$ were purchased from commercial suppliers. Thiadiazole $\mathbf{2}$ were prepared according to known procedures, and their ${ }^{1} \mathrm{H}$ and ${ }^{13} \mathrm{C}$ spectra are identical with those reported. ${ }^{[1]}$

\section{Optimization Studies}

(a)<smiles>O=C(C=[W])c1ccccc1</smiles>

$\mathbf{S 1}, 0.02 \mathrm{mmol}$

(b)<smiles>CCOC(=O)C(=[W])C(=O)c1ccccc1</smiles>

s2, $0.02 \mathrm{mmol}$<smiles>O=CC#Cc1ccccc1</smiles>

1a, $0.04 \mathrm{mmol}$<smiles>O=CC#Cc1ccccc1</smiles>

1a, $0.04 \mathrm{mmol}$ $0 \mathrm{~mol} \%[\mathrm{Rh}(\mathrm{COD}) \mathrm{Cl}]_{2}$
$\underset{\mathrm{PhCl}, 130^{\circ} \mathrm{C}, 24 \mathrm{~h}}{\longrightarrow}$

$$
\text { PhC }
$$

carbene dimerization<smiles>C=C(/C=C/C(=O)c1ccccc1)c1ccccc1</smiles>

S3, $59 \%$ O

$\underset{\mathrm{PhCl}, 130^{\circ} \mathrm{C}, 24 \mathrm{~h}}{\stackrel{10 \mathrm{~mol} \%[\mathrm{Rh}(\mathrm{COD}) \mathrm{Cl}]_{2}}{24 \% \mathrm{DPPF}}}$ Mess

Scheme S1. Reactions of 1a and Diazo compouds S1 and S2

General Procedure for the reactions in Scheme S1. Catalyst $[\mathrm{Rh}(\mathrm{COD}) \mathrm{Cl}]_{2}(10 \mathrm{mg}, 0.020 \mathrm{mmol})$ and ligand DPPF (27 mg, $0.048 \mathrm{mmol}$ ) was added to a flame dried reaction tube. The reaction tube was sealed with a rubber stopper, evacuated and backfilled with nitrogen for three times. A solution of 3-phenylpropiolaldehyde 1a $(52 \mathrm{mg}, 0.400 \mathrm{mmol}, 2 \mathrm{eq})$ in fresh distilled $\mathrm{PhCl}(0.8 \mathrm{~mL}) \mathrm{was}$ injected to the tube. Then 2-diazo-1-phenylethanone (S1, $29.2 \mathrm{mg}, 0.2 \mathrm{mmol}, 1 \mathrm{eq}$ ) or ethyl 2-diazo3-oxo-3-phenylpropanoate (S2, $43.6 \mathrm{mg}, 0.2 \mathrm{mmol}, 1 \mathrm{eq})$ in dry $\mathrm{PhCl}(0.8 \mathrm{~mL})$ was added via a syringe. The mixture was allowed to stir for $24 \mathrm{~h}$. The crude reaction mixture was analyzed by GCMS, LC-MS, and ${ }^{1} \mathrm{H}$ NMR. 
The reaction of $\mathbf{S 1}$ with 1a gave alkene $\mathbf{S 3}$ as major product. The solvent was evaporated under reduced pressure, and the crude mixture was subjected to flash chromatography on silica gel affording the corresponding product $\mathbf{S 3}$ (14 $\mathrm{mg}, 59 \%)$. Known compound, ${ }^{[2]}$ identified by GC-MS (EI): $\mathrm{m} / \mathrm{z}\left(\mathrm{M}^{+}\right.$) caled for $\mathrm{C}_{16} \mathrm{H}_{12} \mathrm{O}_{2}{ }^{+}: 236.08$, found:236.09 (Figure $\mathrm{S1}$ ).

The reaction of $\mathbf{S 2}$ with $\mathbf{1 a}$ only gave a messy mixture.

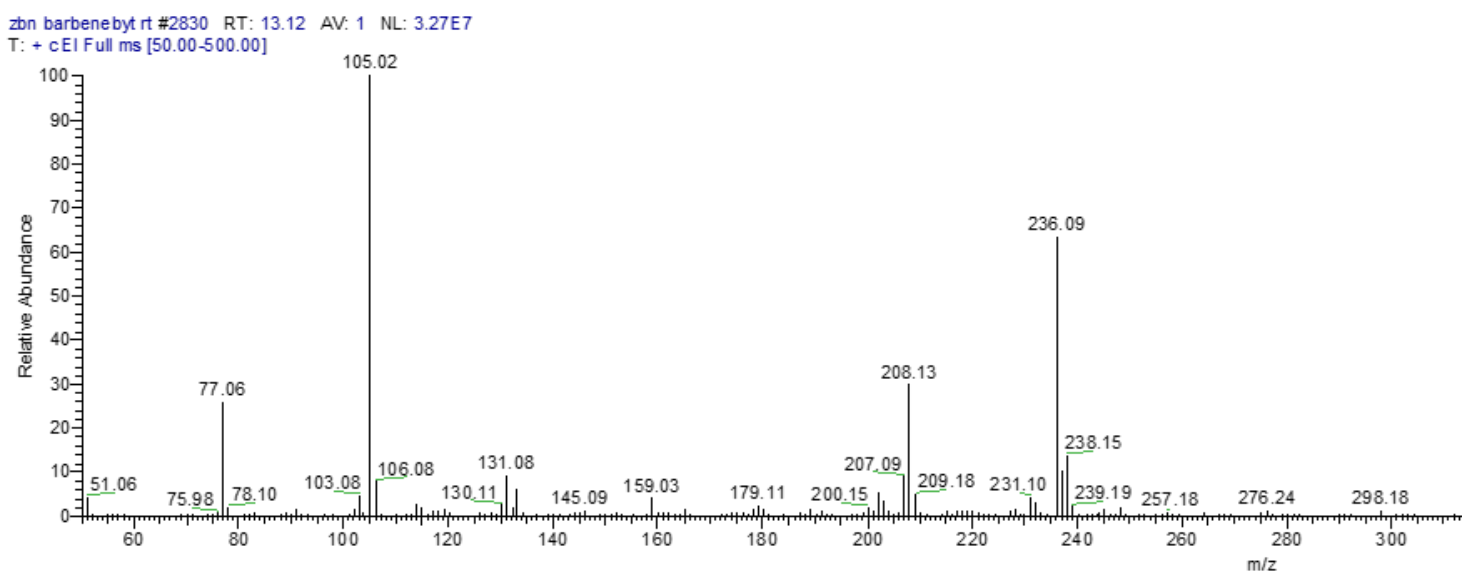

Figure S1. MS spectra of S3.

Table S1. Optimization of the reaction conditions. ${ }^{a}$<smiles></smiles>

DPPM:<smiles>c1ccc(P(CP(c2ccccc2)c2ccccc2)c2ccccc2)cc1</smiles>
DPPE:<smiles>c1ccc(P(CCP(c2ccccc2)c2ccccc2)c2ccccc2)cc1</smiles><smiles>c1ccc(P(c2ccccc2)P(c2ccccc2)c2ccccc2)cc1</smiles>

DPPP:<smiles>c1ccc(P(CCCP(c2ccccc2)c2ccccc2)c2ccccc2)cc1</smiles>
DPPPenta:<smiles>c1ccc(P(CCCCCCP(c2ccccc2)c2ccccc2)c2ccccc2)cc1</smiles><smiles></smiles>

\begin{tabular}{lccllll}
\hline Entry & \multicolumn{1}{c}{ Cat. } & \multicolumn{1}{c}{ Ligand } & Additive & T $\left({ }^{\circ} \mathrm{C}\right)$ & Time (h) & Yield $(\%){ }^{b}$ \\
\hline 1 & {$[\mathrm{Rh}(\mathrm{COD}) \mathrm{Cl}]_{2}(5 \mathrm{~mol} \%)$} & $\mathrm{Ph}_{3} \mathrm{PO}(24 \mathrm{~mol} \%)$ & None & 130 & 6 & 0 \\
2 & {$[\mathrm{Rh}(\mathrm{COD}) \mathrm{Cl}]_{2}(5 \mathrm{~mol} \%)$} & $\mathrm{P}(\mathrm{OEt})_{3}(24 \mathrm{~mol} \%)$ & None & 130 & 6 & 0 \\
3 & {$[\mathrm{Rh}(\mathrm{COD}) \mathrm{Cl}]_{2}(5 \mathrm{~mol} \%)$} & $\mathrm{P}(\mathrm{OPh})_{3}(24 \mathrm{~mol} \%)$ & None & 130 & 6 & 0 \\
4 & {$[\mathrm{Rh}(\mathrm{COD}) \mathrm{Cl}]_{2}(5 \mathrm{~mol} \%)$} & $(\mathrm{PhO})_{3} \mathrm{PO}(24 \mathrm{~mol} \%)$ & None & 130 & 6 & 0 \\
5 & {$[\mathrm{Rh}(\mathrm{COD}) \mathrm{Cl}]_{2}(5 \mathrm{~mol} \%)$} & $(\mathrm{EtO})_{3} \mathrm{PO}(24 \mathrm{~mol} \%)$ & None & 130 & 6 & 0 \\
6 & {$[\mathrm{Rh}(\mathrm{COD}) \mathrm{Cl}]_{2}(5 \mathrm{~mol} \%)$} & $\mathrm{P}\left(\mathrm{C}_{6} \mathrm{~F}_{5}\right)_{3}(24 \mathrm{~mol} \%)$ & None & 130 & 6 & 0 \\
7 & {$[\mathrm{Rh}(\mathrm{COD}) \mathrm{Cl}]_{2}(5 \mathrm{~mol} \%)$} & $\mathrm{PPh}_{3}(24 \mathrm{~mol} \%)$ & None & 130 & 6 & 0 \\
8 & {$[\mathrm{Rh}(\mathrm{COD}) \mathrm{Cl}]_{2}(5 \mathrm{~mol} \%)$} & $\mathrm{DPPM}_{2}(12 \mathrm{~mol} \%)$ & None & 130 & 6 & 0
\end{tabular}




\begin{tabular}{|c|c|c|c|c|c|c|}
\hline 9 & {$[\mathrm{Rh}(\mathrm{COD}) \mathrm{Cl}]_{2}(5 \mathrm{~mol} \%)$} & DPPE (12 mol\%) & None & 130 & 6 & 10 \\
\hline 10 & {$[\mathrm{Rh}(\mathrm{COD}) \mathrm{Cl}]_{2}(5 \mathrm{~mol} \%)$} & DPPP (12 mol\%) & None & 130 & 6 & 36 \\
\hline 11 & {$[\mathrm{Rh}(\mathrm{COD}) \mathrm{Cl}]_{2}(5 \mathrm{~mol} \%)$} & DPPPenta (12 mol\%) & None & 130 & 6 & 30 \\
\hline 12 & {$[\mathrm{Rh}(\mathrm{COD}) \mathrm{Cl}]_{2}(5 \mathrm{~mol} \%)$} & DPPF (12 mol\%) & None & 130 & 6 & 36 \\
\hline 13 & {$[\mathrm{Rh} * \mathrm{Cp}]_{2} \mathrm{Cl}_{2}(5 \mathrm{~mol} \%)$} & DPPF (12 mol\%) & None & 130 & 6 & 40 \\
\hline 14 & {$[\mathrm{Rh}(\mathrm{COD}) \mathrm{Cl}]_{2}(5 \mathrm{~mol} \%)$} & None & None & 130 & 6 & 0 \\
\hline 15 & {$[\mathrm{Rh}(\mathrm{COD}) \mathrm{Cl}]_{2}(5 \mathrm{~mol} \%)$} & DPPF (12 mol\%) & None & 80 & 6 & 23 \\
\hline 16 & {$[\mathrm{Rh}(\mathrm{COD}) \mathrm{Cl}]_{2}(5 \mathrm{~mol} \%)$} & DPPF (12 mol\%) & None & 100 & 6 & 26 \\
\hline 17 & {$[\mathrm{Rh}(\mathrm{COD}) \mathrm{Cl}]_{2}(5 \mathrm{~mol} \%)$} & DPPF (12 mol\%) & None & 150 & 6 & 33 \\
\hline 18 & {$[\mathrm{Rh}(\mathrm{COD}) \mathrm{Cl}]_{2}(5 \mathrm{~mol} \%)$} & DPPF (12 mol\%) & None & 80 & 6 & 10 \\
\hline $19^{c}$ & {$[\mathrm{Rh}(\mathrm{COD}) \mathrm{Cl}]_{2}(5 \mathrm{~mol} \%)$} & DPPF (12 mol\%) & None & 110 & 6 & 36 \\
\hline 20 & {$[\mathrm{Rh}(\mathrm{COD}) \mathrm{Cl}]_{2}(5 \mathrm{~mol} \%)$} & DPPF (12 mol\%) & $\begin{array}{l}\mathrm{AgNO}_{3}(12 \\
\mathrm{mol} \%)\end{array}$ & 130 & 6 & 5 \\
\hline 21 & {$[\mathrm{Rh}(\mathrm{COD}) \mathrm{Cl}]_{2}(5 \mathrm{~mol} \%)$} & DPPF (12 mol\%) & $\begin{array}{l}\text { AgOAc } \quad(12 \\
\text { mol\%) }\end{array}$ & 130 & 6 & 42 \\
\hline 22 & {$[\mathrm{Rh}(\mathrm{COD}) \mathrm{Cl}]_{2}(5 \mathrm{~mol} \%)$} & DPPF (12 mol\%) & $\begin{array}{l}\mathrm{Ag}_{2} \mathrm{O} \\
\mathrm{mol} \%)\end{array}$ & 130 & 6 & 20 \\
\hline 23 & {$[\mathrm{Rh}(\mathrm{COD}) \mathrm{Cl}]_{2}(5 \mathrm{~mol} \%)$} & DPPF (12 mol\%) & $\begin{array}{l}\mathrm{AgSbF}_{6} \quad(12 \\
\mathrm{mol} \%)\end{array}$ & 130 & 6 & 30 \\
\hline 24 & {$[\mathrm{Rh}(\mathrm{COD}) \mathrm{Cl}]_{2}(5 \mathrm{~mol} \%)$} & DPPF (12 mol\%) & $\begin{array}{l}\mathrm{CF}_{3} \mathrm{SO}_{2} \mathrm{Ag} \\
(12 \mathrm{~mol} \%)\end{array}$ & 130 & 6 & 21 \\
\hline 25 & {$[\mathrm{Rh}(\mathrm{COD}) \mathrm{Cl}]_{2}(5 \mathrm{~mol} \%)$} & DPPF (12 mol\%) & $\begin{array}{l}\mathrm{AgBF}_{4} \\
\text { mol\%) }\end{array}$ & 130 & 6 & 0 \\
\hline 26 & {$[\mathrm{Rh}(\mathrm{COD}) \mathrm{Cl}]_{2}(5 \mathrm{~mol} \%)$} & DPPF (12 mol\%) & $\begin{array}{l}\text { NaBARF (12 } \\
\text { mol\%) }\end{array}$ & 130 & 6 & 14 \\
\hline 27 & {$[\mathrm{Rh}(\mathrm{COD}) \mathrm{Cl}]_{2}(5 \mathrm{~mol} \%)$} & DPPF (12 mol\%) & $\begin{array}{l}\mathrm{AgNTf}_{2} \quad(12 \\
\text { mol\%) }\end{array}$ & 130 & 6 & 17 \\
\hline $28^{d}$ & {$[\mathrm{Rh}(\mathrm{COD}) \mathrm{Cl}]_{2}(5 \mathrm{~mol} \%)$} & DPPF (12 mol\%) & $\begin{array}{l}\mathrm{AgSbF}_{6} \quad(12 \\
\mathrm{mol} \%)\end{array}$ & 130 & 12 & 31 \\
\hline 29 & {$[\mathrm{Rh}(\mathrm{COD}) \mathrm{Cl}]_{2}(10 \mathrm{~mol} \%)$} & DPPF (24 mol\%) & $\begin{array}{l}\mathrm{AgSbF}_{6} \quad(24 \\
\mathrm{mol} \%)\end{array}$ & 130 & 6 & 38 \\
\hline 30 & {$[\mathrm{Rh}(\mathrm{COD}) \mathrm{Cl}]_{2}(10 \mathrm{~mol} \%)$} & DPPF (24 mol\%) & $\begin{array}{l}\mathrm{AgSbF}_{6} \quad(24 \\
\mathrm{mol} \%)\end{array}$ & 130 & 12 & 45 \\
\hline 31 & $\begin{array}{l}{[\mathrm{Rh}(\mathrm{COD}) \mathrm{Cl}]_{2}} \\
\mathrm{~mol} \%)\end{array}$ & DPPF (24 mol\%) & $\begin{array}{l}\mathrm{AgSbF}_{6} \\
\text { mol\%) }\end{array}$ & 130 & 24 & $61^{\mathrm{e}}$ \\
\hline 32 & {$[\mathrm{Rh}(\mathrm{COD}) \mathrm{Cl}]_{2}(10 \mathrm{~mol} \%)$} & DPPF (24 mol\%) & $\begin{array}{l}\mathrm{AgSbF}_{6} \quad(24 \\
\left.\mathrm{mol}^{\circ}\right)\end{array}$ & 130 & 48 & $60^{\mathrm{e}}$ \\
\hline
\end{tabular}




\begin{tabular}{|c|c|c|c|c|c|c|c|}
\hline 33 & $\mathrm{Cu}(\mathrm{acac})_{2}(10 \mathrm{~mol} \%)$ & DPPF (24 mol\%) & $\begin{array}{l}\mathrm{AgSbF}_{6} \\
\mathrm{~mol} \%)\end{array}$ & (24 & 130 & 24 & 13 \\
\hline 34 & {$[\operatorname{Ir}(\mathrm{COD}) \mathrm{Cl}]_{2}(10 \mathrm{~mol} \%)$} & DPPF (24 mol\%) & $\begin{array}{l}\mathrm{AgSbF}_{6} \\
\mathrm{~mol} \%)\end{array}$ & & 130 & 24 & 27 \\
\hline
\end{tabular}

[a] The reactions were run on a 0.1-mmol scale in chlorobenzene under $\mathrm{N}_{2}$ atmosphere. The ratio of $\mathbf{2 a}(0.1 \mathrm{mmol})$ and $\mathbf{1 a}(0.2 \mathrm{mmol})$ was $1: 2$. [b] The yields were determined by ${ }^{1} \mathrm{H}$ NMR with 1,3,5-trimethoxybenzene as an internal standard. [c] Toluene was used as a solvent. ${ }^{d}$ Three equivalents of $\mathbf{1 a}$ was used. ${ }^{e}$ Isolated yields after colunmn chromatography.

\section{Experimental Procedures}

\subsection{General procedure for synthesis of 2-ynals 1}

$\operatorname{Method~A} \mathbf{A}^{[3 \mathrm{~b}, \mathrm{c}, \mathrm{d}]}$

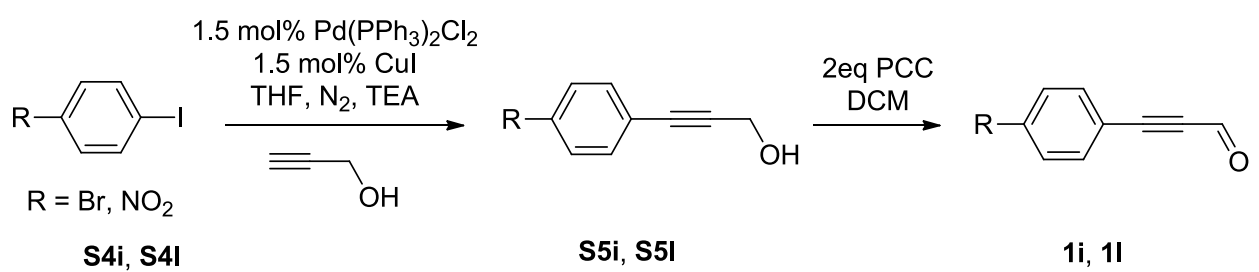

Scheme S2. Synthesis of ynal via Sonogashira coupling and Jones oxidation

a) A mixture of $\mathbf{S 4 i}(1.13 \mathrm{~g}, 4.00 \mathrm{mmol})$ or $\mathbf{S 4 l}(1.00 \mathrm{~g}, 4.00 \mathrm{mmol}), \mathrm{Pd}\left(\mathrm{PPh}_{3}\right)_{2} \mathrm{Cl}_{2}(42 \mathrm{mg}, 0.06$ $\mathrm{mmol})$, $\mathrm{CuI}(11 \mathrm{mg}, 0.06 \mathrm{mmol})$ was dissolved in dry THF $(10 \mathrm{~mL})$ under $\mathrm{N}_{2}$ atmosphere. After the addition of $\mathrm{Et}_{3} \mathrm{~N}$ (1.62 g, $\left.2.3 \mathrm{~mL}, 16 \mathrm{mmol}\right)$ and prop-2-yn-1-ol ( $\left.0.26 \mathrm{~mL}, 4.5 \mathrm{mmol}\right)$ via syringes, the mixture was allowed to be stirred at room temperature overnight. After removal of the solvent in vacuo, the crude mixture was purified by column chromatography on silica gel affording the correspondintg products $\mathbf{S 5 i}$ and $\mathbf{S 5 I}$ as white solid in $74 \%$ and $75 \%$ yields.

b) The product 3-(4-bromophenyl)-prop-2-yn-1-ol S5i (0.63 g, $3.0 \mathrm{mmol}, 1 \mathrm{eq}$ ) or 3-(4-nitrophenyl)prop-2-yn-1-ol S5I (0.53 g, $3.0 \mathrm{mmol}, 1 \mathrm{eq})$ was dissolved in dry DCM (20 mL). Then PCC (1.29 $\mathrm{g}, 6.0 \mathrm{mmol}, 2 \mathrm{eq})$ was added portionally to the solution. And then diatomite $(1.29 \mathrm{~g})$ was added to the solution.The mixture was stirred at room temperature for 2 hours under nitrogen. After filtering through a pad of silica gel with DCM as eluent and removal of the solvent under reduced pressure, 1i and 11 were obtained after purafication by column chromatography as solid in $46 \%$ and $67 \%$ yields, respectively.

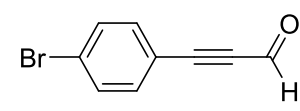

$1 \mathbf{1}$

\section{3-(4-Bromophenyl)propiolaldehyde (1i) $)^{[3 a]}$}

White solid, $\mathrm{R}_{f}=0.20\left(\mathrm{CH}_{2} \mathrm{Cl}_{2} / \mathrm{PE} 1: 1\right)$, M.p. $102-103{ }^{\circ} \mathrm{C}, 284 \mathrm{mg}$, yield $34 \%$ over 2 steps. ${ }^{1} \mathbf{H}$ NMR $\left(400 \mathrm{MHz}, \mathrm{CDCl}_{3}\right) \delta 9.39(\mathrm{~s}, 1 \mathrm{H}), 7.56-7.52$ (m, 2H), $7.46-7.43(\mathrm{~m}, 2 \mathrm{H}) .{ }^{13} \mathbf{C}$ NMR $\left(101 \mathrm{MHz}, \mathrm{CDCl}_{3}\right) \delta 176.5,134.5$, 132.2, 126.2, 118.3, 93.6, 89.0. 


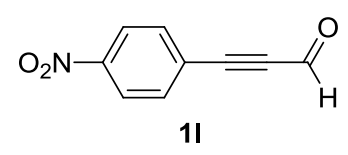

\section{3-(4-Nitrophenyl)propiolaldehyde $(11)^{[3 a]}$}

Yellow solid, $\mathrm{R}_{f}=0.14\left(\mathrm{CH}_{2} \mathrm{Cl}_{2} / \mathrm{PE} \mathrm{1:1)}\right.$, M.p. $120-122{ }^{\circ} \mathrm{C}, 350 \mathrm{mg}$, yield $50 \%$ over 2 steps. ${ }^{1} \mathbf{H}$ NMR $\left(400 \mathrm{MHz}, \mathrm{CDCl}_{3}\right) \delta 9.44(\mathrm{~s}, 1 \mathrm{H}), 8.28-8.24$ (m, 2H), $7.77-7.73(\mathrm{~m}, 2 \mathrm{H}) .{ }^{13} \mathbf{C}$ NMR $\left(101 \mathrm{MHz}, \mathrm{CDCl}_{3}\right) \delta 176.1,148.8$, 133.9, 126.0, 123.9, 90.8, 90.6.

Method B ${ }^{[4 a]}$

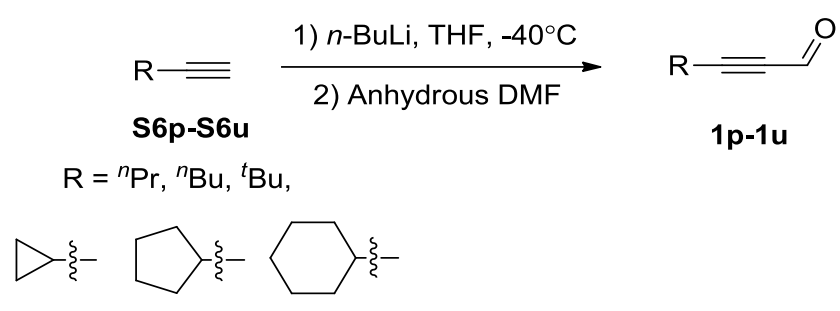

Scheme S3. Synthesis of ynal with terminal alkyne and DMF

A flame dried flask was charged with a solution of terminal alkyne (S6p-S6u, 5 mmol) in dry THF $(10 \mathrm{~mL})$ under nitrogen atomsphere. $n$-Butyllithium (1.6 M in hexane, $6.25 \mathrm{~mL}, 10 \mathrm{mmol}$ ) was added dropwise to the solution at $-40{ }^{\circ} \mathrm{C}$. Then the mixture was stirred for $30 \mathrm{~min}$, after that dry DMF $(1.16 \mathrm{~mL}, 15.0 \mathrm{mmol})$ was dropwise added, and then the mixture was allowed to warm up to room temperature. The solution was stirred for another $20 \mathrm{~min}$. Then the mixture was poured into ice water and acidified with concentrated hydrochloric acid. The mixture was then neutralized with $\mathrm{NaHCO}_{3}$ until $\mathrm{pH}$ of the solution reached about 7. The organic layer was separated and the aqueous layer was extracted with DCM $(3 \times 20 \mathrm{~mL})$. The combined organic layers were dried over $\mathrm{Na}_{2} \mathrm{SO}_{4}$, filtered, and concentrated in vacuo. Pure 1p-1u can be obtained in 50-84\% yields as yellow oil by column chromatography seperation.
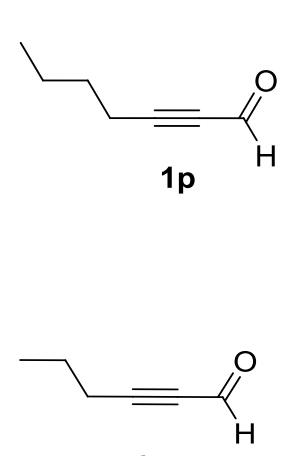

$1 q$

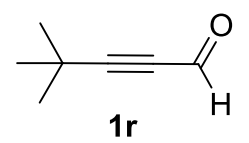

Hept-2-ynal (1p) ${ }^{[4 b]}$

Yellow oil, $\mathrm{R}_{f}=0.40\left(\mathrm{CH}_{2} \mathrm{Cl}_{2} / \mathrm{PE} 1: 1\right), 286 \mathrm{mg}$, yield 52\%. ${ }^{1} \mathbf{H} \mathbf{N M R}(400 \mathrm{MHz}$, $\left.\mathrm{CDCl}_{3}\right) \delta 9.14(\mathrm{~s}, 1 \mathrm{H}), 2.38(\mathrm{td}, J=7.0,1.0 \mathrm{~Hz}, 2 \mathrm{H}), 1.58-1.51(\mathrm{~m}, 2 \mathrm{H}), 1.45$ $-1.37(\mathrm{~m}, 2 \mathrm{H}), 0.89$ (t, $J=7.0 \mathrm{~Hz}, 3 \mathrm{H}) .{ }^{13} \mathbf{C ~ N M R}\left(101 \mathrm{MHz}, \mathrm{CDCl}_{3}\right) \delta 177.2$, 99.3, 81.6, 29.5, 21.9, 18.8, 13.4.

\section{Hex-2-ynal (1q) $)^{[4 c]}$}

Yellow oil, $\mathrm{R}_{f}=0.40\left(\mathrm{CH}_{2} \mathrm{Cl}_{2} / \mathrm{PE} 1: 1\right), 307 \mathrm{mg}$, yield 64\%. ${ }^{1} \mathbf{H} \mathbf{N M R}(400 \mathrm{MHz}$, $\left.\mathrm{CDCl}_{3}\right) \delta 8.68(\mathrm{~s}, 1 \mathrm{H}), 3.06(\mathrm{t}, J=7.2 \mathrm{~Hz}, 2 \mathrm{H}), 1.84-1.74(\mathrm{~m}, 2 \mathrm{H}), 1.01(\mathrm{t}, J$ $=7.2 \mathrm{~Hz}, 3 \mathrm{H}) .{ }^{13} \mathbf{C}$ NMR $\left(101 \mathrm{MHz}, \mathrm{CDCl}_{3}\right) \delta 199.1,102.2,78.3,21.8,17.5$, 13.7 .

4,4-Dimethylpent-2-ynal $(1 \mathrm{r})^{[4 \mathrm{~b}]}$

Yellow oil, $\mathrm{R}_{f}=0.30\left(\mathrm{CH}_{2} \mathrm{Cl}_{2} / \mathrm{PE} 1: 1\right), 275 \mathrm{mg}$, yield 50\%. ${ }^{1} \mathbf{H} \mathbf{~ N M R}(400 \mathrm{MHz}$, $\left.\mathrm{CDCl}_{3}\right) \delta 9.17(\mathrm{~s}, 1 \mathrm{H}), 1.28(\mathrm{~s}, 9 \mathrm{H}) .{ }^{13} \mathrm{C} \mathbf{N M R}\left(101 \mathrm{MHz}, \mathrm{CDCl}_{3}\right) \delta$ 177.4, $106.3,80.2,29.9,27.9$. 


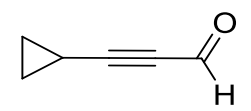

$1 \mathrm{~s}$

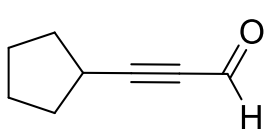

$1 \mathrm{t}$

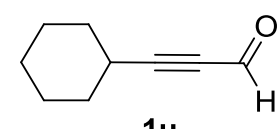

$1 \mathrm{u}$
3-Cyclopropylpropiolaldehyde (1s) $)^{[\mathrm{dd}]}$

Yellow oil, $\mathrm{R}_{f}=0.30\left(\mathrm{CH}_{2} \mathrm{Cl}_{2} / \mathrm{PE} 1: 1\right), 348 \mathrm{mg}$, yield $74 \%$. ${ }^{1} \mathbf{H}$ NMR $(400 \mathrm{MHz}$, $\left.\mathrm{CDCl}_{3}\right) \delta 9.11(\mathrm{~s}, 1 \mathrm{H}), 1.49-1.40(\mathrm{~m}, 1 \mathrm{H}), 1.08-0.92(\mathrm{~m}, 4 \mathrm{H}) .{ }^{13} \mathbf{C} \mathbf{N M R}(101$ $\left.\mathrm{MHz}, \mathrm{CDCl}_{3}\right) \delta$ 176.9, 104.0, 77.9, 10.3, 0.1 .

\section{3-Cyclopentylpropiolaldehyde (1t) ${ }^{[4 \mathrm{e}]}$}

Yellow oil, $\mathrm{R}_{f}=0.30\left(\mathrm{CH}_{2} \mathrm{Cl}_{2} / \mathrm{PE} 1: 1\right), 500 \mathrm{mg}$, yield $82 \% .{ }^{1} \mathbf{H}$ NMR $(400$ $\left.\mathrm{MHz}, \mathrm{CDCl}_{3}\right) \delta 9.13(\mathrm{~s}, 1 \mathrm{H}), 2.80-2.73(\mathrm{~m}, 1 \mathrm{H}), 2.02-1.88(\mathrm{~m}, 2 \mathrm{H}), 1.74-$ $1.62(\mathrm{~m}, 4 \mathrm{H}), 1.61-1.52(\mathrm{~m}, 2 \mathrm{H}) .{ }^{13} \mathbf{C}$ NMR $\left(101 \mathrm{MHz}, \mathrm{CDCl}_{3}\right) \delta$ 177.2, 103.3, 81.1, 33.0, 30.0, 25.1 .

\section{3-Cyclohexylpropiolaldehyde (1u) $)^{[\mathrm{dd}]}$}

Yellow oil, $\mathrm{R}_{f}=0.32\left(\mathrm{CH}_{2} \mathrm{Cl}_{2} / \mathrm{PE} 1: 1\right), 571 \mathrm{mg}$, yield $84 \% .{ }^{1} \mathrm{H}$ NMR $(400 \mathrm{MHz}$, $\left.\mathrm{CDCl}_{3}\right) \delta 9.18(\mathrm{~s}, 1 \mathrm{H}), 2.67-2.50(\mathrm{~m}, 1 \mathrm{H}), 1.93-1.79(\mathrm{~m}, 2 \mathrm{H}), 1.73-1.66$ $(\mathrm{m}, 2 \mathrm{H}), 1.62-1.46(\mathrm{~m}, 3 \mathrm{H}), 1.37-1.30(\mathrm{~m}, 3 \mathrm{H}) .{ }^{13} \mathbf{C} \mathbf{~ N M R}\left(101 \mathrm{MHz}, \mathrm{CDCl}_{3}\right)$ $\delta$ 177.4, 102.8, 81.6, 31.4, 29.3, 25.5, 24.6.
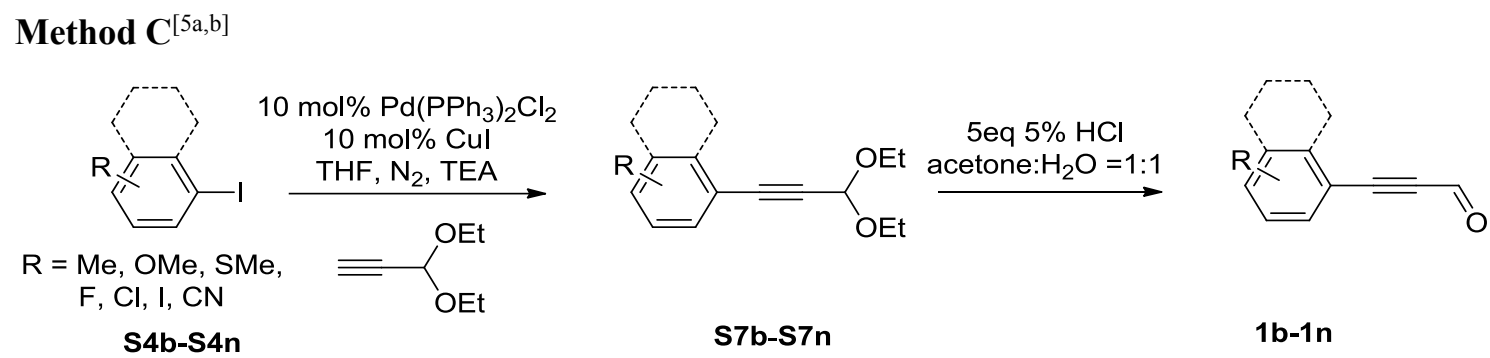

Scheme S4. Synthesis of ynal via Sonogashira coupling and acetal hydrolysis reaction

a) A mixture of S4b-S4n (5.0 mmol), 3,3-diethoxyprop-1-yne (1.0 mL, $7 \mathrm{mmol})$, triethylamine (3.0 $\mathrm{mL}$ ), and $\mathrm{Pd}\left(\mathrm{PPh}_{3}\right)_{2} \mathrm{Cl}_{2}(350 \mathrm{mg}, 0.5 \mathrm{mmol})$, $\mathrm{CuI}(95 \mathrm{mg}, 0.5 \mathrm{mmol})$ in $15 \mathrm{~mL}$ of THF was stirred in open air at $25^{\circ} \mathrm{C}$ for $4 \mathrm{~h}$. The solvent was removed in vacuo, then the residue was diluted with water $(15 \mathrm{~mL})$ and DCM $(15 \mathrm{~mL})$. After extracting with DCM $(3 \times 20 \mathrm{~mL})$, the combined organic phase was dried by $\mathrm{Na}_{2} \mathrm{SO}_{4}$ and filtrated. The filtrate was evaporated. Chromatography of the residue on silica gel provided the corresponding product $\mathbf{S 7 b - S 7 n}$.

b) S7a-S7n was dissolved in mixed solvent $(20 \mathrm{~mL})$ of acetone and $\mathrm{H}_{2} \mathrm{O}(v: v=1: 1)$, then 5 equivalents of $5 \% \mathrm{HCl}(15 \mathrm{~mL})$ was added to the solution, and the mixture was allowed to stand overnight at room temperature. Upon completion of the reaction, acetone was removed in vacuum, and the aqueous layer was extracted with DCM $(3 \times 20 \mathrm{~mL})$. The combined organic layers were dried over anhydrous $\mathrm{Na}_{2} \mathrm{SO}_{4}$, and concentrated in vacuo. Purification of the residue by column chromatography gave $\mathbf{1 b}-\mathbf{1 n}$.

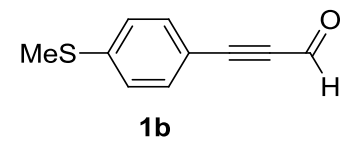

\section{3-(4-(Methylthio)phenyl)propiolaldehyde (1b)}

Yellow oil, $\mathrm{R}_{f}=0.20\left(\mathrm{CH}_{2} \mathrm{Cl}_{2} / \mathrm{PE} 1: 1\right), 457 \mathrm{mg}$, yield $52 \%$ over 2 steps. ${ }^{1} \mathbf{H}$ NMR $\left(400 \mathrm{MHz}, \mathrm{CDCl}_{3}\right) \delta 9.43(\mathrm{~s}, 1 \mathrm{H}), 7.52(\mathrm{~d}, J=8.3 \mathrm{~Hz}, 2 \mathrm{H}), 7.25$ (d, $J=8.3 \mathrm{~Hz}, 2 \mathrm{H}), 2.53(\mathrm{~s}, 3 \mathrm{H}) .{ }^{13} \mathbf{C} \mathbf{N M R}\left(101 \mathrm{MHz}, \mathrm{CDCl}_{3}\right) \delta 176.6,144.3$, 133.6, 125.4, 115.0, 95.6, 89.1, 14.8. HRMS (ESI) $\mathrm{m} / \mathrm{z}[\mathrm{M}+\mathrm{H}]^{+}$calcd for 
$\mathrm{C}_{10} \mathrm{H}_{9} \mathrm{OS}^{+}:$177.0369, found: 177.0371.
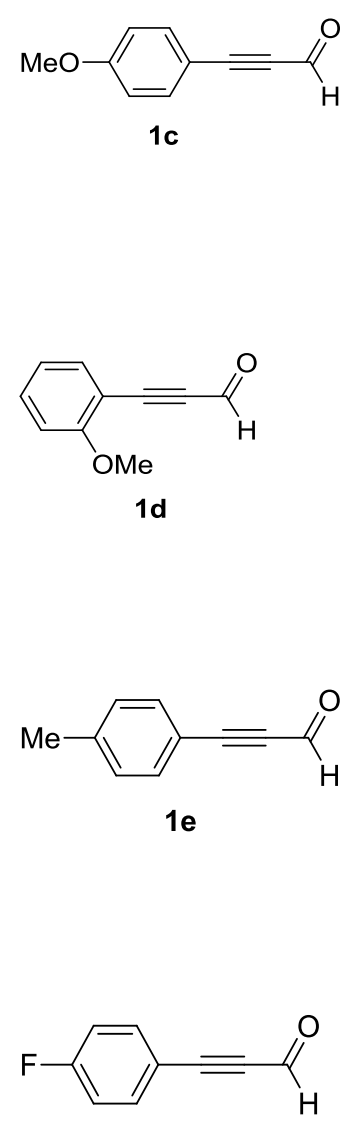

$1 f$

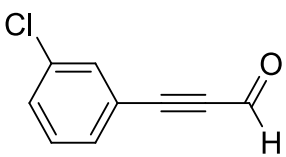

1g

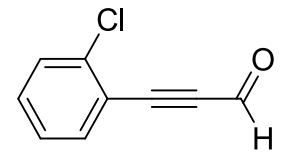

$1 \mathrm{~h}$

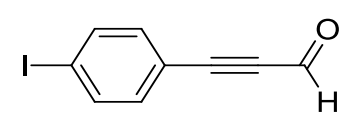

1j

\section{3-(4-Methoxyphenyl)propiolaldehyde $(1 \mathrm{c})^{[5 c]}$}

Yellow oil, $\mathrm{R}_{f}=0.14\left(\mathrm{CH}_{2} \mathrm{Cl}_{2} / \mathrm{PE} 1: 1\right), 488 \mathrm{mg}$, yield $61 \%$ over 2 steps. ${ }^{1} \mathbf{H}$ NMR (400 MHz, $\left.\mathrm{CDCl}_{3}\right) \delta 9.37(\mathrm{~s}, 1 \mathrm{H}), 7.55-7.53(\mathrm{~m}, 2 \mathrm{H}), 6.90-6.88$ (m, 2H), 3.83 (s, 3H). ${ }^{13} \mathbf{C}$ NMR (101 MHz, $\left.\mathrm{CDCl}_{3}\right) \delta 176.7,162.1,135.4$, $114.5,111.1,96.5,88.7,55.4$.

\section{3-(2-Methoxyphenyl)propiolaldehyde $(1 \mathrm{~d})^{[5 \mathrm{~d}]}$}

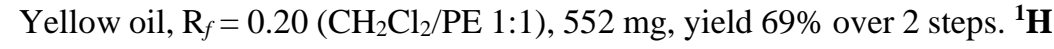
NMR (400 MHz, $\left.\mathrm{CDCl}_{3}\right) \delta 9.46(\mathrm{~s}, 1 \mathrm{H}), 7.54(\mathrm{~d}, J=7.6 \mathrm{~Hz}, 1 \mathrm{H}), 7.49-$ $7.41(\mathrm{~m}, 1 \mathrm{H}), 6.99-6.89(\mathrm{~m}, 2 \mathrm{H}), 3.92(\mathrm{~s}, 3 \mathrm{H}) .{ }^{13} \mathrm{C}$ NMR $(101 \mathrm{MHz}$, $\left.\mathrm{CDCl}_{3}\right) \delta 176.8,161.7,135.3,133.1,120.7,110.9,108.7,92.7,92.4,55.9$.

3-(p-Tolyl)propiolaldehyde (1e) ${ }^{[5 \mathrm{e}]}$

Yellow oil, $\mathrm{R}_{f}=0.25\left(\mathrm{CH}_{2} \mathrm{Cl}_{2} / \mathrm{PE} 1: 1\right), 461 \mathrm{mg}$, yield $64 \%$ over 2 steps. ${ }^{1} \mathbf{H}$ NMR (400 MHz, $\left.\mathrm{CDCl}_{3}\right) \delta 9.41(\mathrm{~s}, 1 \mathrm{H}), 7.49(\mathrm{~d}, J=8.1 \mathrm{~Hz}, 2 \mathrm{H}), 7.20$ (d, $J=8.1 \mathrm{~Hz}, 2 \mathrm{H}), 2.39$ (s, 3H). ${ }^{13} \mathbf{C}$ NMR $\left(101 \mathrm{MHz}, \mathrm{CDCl}_{3}\right) \delta 176.6,142.0$, 133.2, 129.4, 116.1, 95.7, 88.3, 21.6.

\section{3-(4-Fluorophenyl)propiolaldehyde (1f $)^{[5 c]}$}

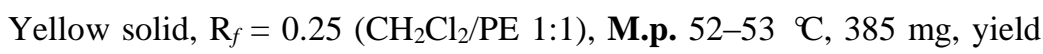
$52 \%$ over 2 steps. 'H NMR $\left(400 \mathrm{MHz}, \mathrm{CDCl}_{3}\right) \delta 9.38(\mathrm{~s}, 1 \mathrm{H}), 7.57$ (dd, $J$ $=9.0,5.0 \mathrm{~Hz}, 2 \mathrm{H}), 7.03(\mathrm{t}, J=9.0 \mathrm{~Hz}, 2 \mathrm{H}) .{ }^{13} \mathbf{C ~ N M R}\left(101 \mathrm{MHz}, \mathrm{CDCl}_{3}\right)$ $\delta 176.6,164.3(\mathrm{~d}, J=250.8 \mathrm{~Hz}), 135.6(\mathrm{~d}, J=9.0 \mathrm{~Hz}), 116.4(\mathrm{~d}, J=22.4$ $\mathrm{Hz}), 115.6(\mathrm{~d}, J=3.5 \mathrm{~Hz}), 94.0,91.3$.

\section{3-(3-Chlorophenyl)propiolaldehyde $(\mathbf{1 g})^{[5 f]}$}

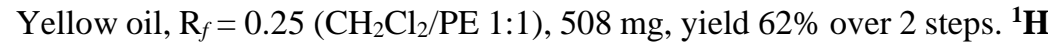
NMR $\left(400 \mathrm{MHz}, \mathrm{CDCl}_{3}\right) \delta 9.48(\mathrm{~s}, 1 \mathrm{H}), 7.65(\mathrm{dd}, J=7.6,1.4 \mathrm{~Hz}, 1 \mathrm{H})$, $7.46(\mathrm{dt}, J=8.0,2.0 \mathrm{~Hz}, 1 \mathrm{H}), 7.42(\mathrm{dt}, J=8.0,2.0 \mathrm{~Hz}, 1 \mathrm{H}), 7.30(\mathrm{dt}, J=$ 8.0, $2.0 \mathrm{~Hz}, 1 \mathrm{H}) .{ }^{13} \mathrm{C}$ NMR $\left(101 \mathrm{MHz}, \mathrm{CDCl}_{3}\right) \delta 176.7,140.4,137.8$, 135.2, 132.4, 129.9, 127.0, 92.3, 91.1.

3-(2-Chlorophenyl)propiolaldehyde $(1 \mathrm{~h})^{[5 \mathrm{~g}]}$

Yellow oil, $\mathrm{R}_{f}=0.25\left(\mathrm{CH}_{2} \mathrm{Cl}_{2} / \mathrm{PE} 1: 1\right), 475 \mathrm{mg}$, yield $58 \%$ over 2 steps. ${ }^{1} \mathbf{H}$ NMR $\left(400 \mathrm{MHz}, \mathrm{CDCl}_{3}\right) \delta 9.48(\mathrm{~s}, 1 \mathrm{H}), 7.62(\mathrm{~d}, J=7.7 \mathrm{~Hz}, 1 \mathrm{H}), 7.51-$ $7.37(\mathrm{~m}, 2 \mathrm{H}), 7.30(\mathrm{t}, J=7.5 \mathrm{~Hz}, 1 \mathrm{H}) .{ }^{13} \mathbf{C} \mathbf{N M R}\left(101 \mathrm{MHz}, \mathrm{CDCl}_{3}\right) \delta$ $176.5,137.7,135.0,132.2,129.8,126.8,119.8,92.1,90.9$.

\section{3-(4-Iodophenyl)propiolaldehyde $(\mathbf{1 j})^{[5 \mathbf{h}]}$}

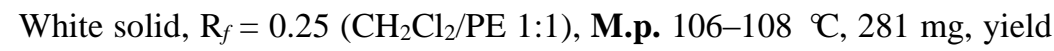
$22 \%$ over 2 steps. ${ }^{1} \mathbf{H}$ NMR $\left(400 \mathrm{MHz}, \mathrm{CDCl}_{3}\right) \delta 9.40(\mathrm{~s}, 1 \mathrm{H}), 7.77$ (d, $J=$ 
$8.2 \mathrm{~Hz}, 2 \mathrm{H}), 7.31(\mathrm{~d}, J=8.2 \mathrm{~Hz}, 2 \mathrm{H}) .{ }^{13} \mathbf{C ~ N M R}\left(101 \mathrm{MHz}, \mathrm{CDCl}_{3}\right) \delta 176.5$, 138.1, 134.3, 118.8, 98.4, 93.7, 89.2.

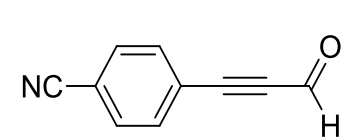

$1 \mathrm{k}$

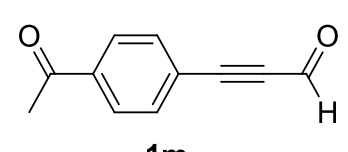

$1 \mathrm{~m}$

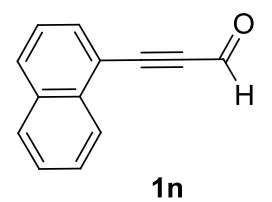

\section{(4-Cyanophenyl)propynal (1k) ${ }^{[5 i]}$}

White solid, $\mathrm{R}_{f}=0.15\left(\mathrm{CH}_{2} \mathrm{Cl}_{2} / \mathrm{PE} 1: 1\right)$, M.p. $100-102{ }^{\circ} \mathrm{C}, 333 \mathrm{mg}$, yield $43 \%$ over 2 steps. ${ }^{1} \mathbf{H}$ NMR (400 $\left.\mathrm{MHz} \mathrm{CDCl}_{3}\right) \delta 9.35$ (s, 1H), $7.72-7.67$ (m, 4H). ${ }^{13} \mathbf{C} \mathbf{N M R}\left(101 \mathrm{MHz}, \mathrm{CDCl}_{3}\right) \delta 176.1,133.4,132.3,124.2,117.7$, 114.6, 91.2, 90.3 .

\section{3-(4-Acetylphenyl)propiolaldehyde $(1 \mathrm{~m})^{[5 \mathrm{~d}]}$}

Yellow solid, $\mathrm{R}_{f}=0.10\left(\mathrm{CH}_{2} \mathrm{Cl}_{2} / \mathrm{PE} \mathrm{1:1)}\right.$, M.p. $80-82{ }^{\circ} \mathrm{C}, 490 \mathrm{mg}$, yield $57 \%$ over 2 steps. ${ }^{1} \mathbf{H}$ NMR $\left(400 \mathrm{MHz}, \mathrm{CDCl}_{3}\right) \delta 9.45(\mathrm{~s}, 1 \mathrm{H}), 7.98(\mathrm{~d}, J=$ $8.2 \mathrm{~Hz}, 2 \mathrm{H}), 7.69(\mathrm{~d}, J=8.2 \mathrm{~Hz}, 2 \mathrm{H}), 2.63$ (s, 3H). ${ }^{13} \mathbf{C}$ NMR $(101 \mathrm{MHz}$, $\left.\mathrm{CDCl}_{3}\right) \delta 197.0,176.4,138.5,133.3,128.4,124.0,92.9,89.8,26.7$.

\section{3-(Naphthalen-1-yl)propiolaldehyde $(1 \mathrm{n})^{[4 \mathrm{~b}]}$}

Orange oil, $\mathrm{R}_{f}=0.25\left(\mathrm{CH}_{2} \mathrm{Cl}_{2} / \mathrm{PE} 1: 1\right), 729 \mathrm{mg}$, yield $81 \%$ over 2 steps. ${ }^{1} \mathbf{H}$ NMR (400 MHz, $\left.\mathrm{CDCl}_{3}\right) 9.56(\mathrm{~s}, 1 \mathrm{H}), 8.32(\mathrm{dd}, J=8.5,0.5 \mathrm{~Hz}, 1 \mathrm{H}), 7.98$ $(\mathrm{d}, J=8.0 \mathrm{~Hz}, 1 \mathrm{H}), 7.90-7.86(\mathrm{~m}, 2 \mathrm{H}), 7.65-7.61(\mathrm{~m}, 1 \mathrm{H}), 7.59-7.54$ $(\mathrm{m}, 1 \mathrm{H}), 7.50-7.46(\mathrm{~m}, 1 \mathrm{H}) .{ }^{13} \mathbf{C} \mathbf{N M R}\left(101 \mathrm{MHz}, \mathrm{CDCl}_{3}\right) \delta$ 176.6, 133.7, 133.6, 133.0, 132.1, 128.6, 127.9, 127.1, 125.6, 125.2, 116.9, 93.4, 93.1.

S7o was synthesized from 5-bromobenzo[b]thiophene instead of 5-iodobenzo[b]thiophene. $\mathrm{Pd}\left(\mathrm{PPh}_{3}\right)_{2} \mathrm{Cl}_{2} \quad(350 \mathrm{mg}, 0.5 \mathrm{mmol}, 0.1 \mathrm{eq}), \mathrm{CuI}(95 \mathrm{mg}, 0.5 \mathrm{mmol}, 0.1 \mathrm{eq})$ and $5-$ bromobenzo[b]thiophene $(1.07 \mathrm{~g}, 5 \mathrm{mmol}, 1 \mathrm{eq})$ was loaded to a dry flask, then the flask was flushed with nitrogen, after that 3,3-diethoxyprop-1-yne $(1.00 \mathrm{~mL}, 7 \mathrm{mmol})$, triethylamine $(3.0 \mathrm{~mL})$ and 15 $\mathrm{mL}$ dry THF were added to the mixture. The reaction mixture was stirred at $70{ }^{\circ} \mathrm{C}$ for $4 \mathrm{~h}$. After cooling to room temperature, the solvent was removed in vacuo, then the residue was diluted with water $(15 \mathrm{~mL})$ and DCM $(15 \mathrm{~mL})$, and then extracted with DCM $(3 \times 20 \mathrm{~mL})$, the combined organic layers were dried by $\mathrm{Na}_{2} \mathrm{SO}_{4}$, after filtration the filtrate was evaporated. Chromatography of the residue on silica gel provided the corresponding product $\mathbf{S 7 0}$ in $73 \%$ yield.<smiles>O=CC#Cc1ccc2sccc2c1</smiles>

10

\section{3-(Benzo $[b]$ thiophen-5-yl)propiolaldehyde (10)}

White solid, $\mathrm{R}_{f}=0.35\left(\mathrm{CH}_{2} \mathrm{Cl}_{2} / \mathrm{PE} \mathrm{1:1)}\right.$, M.p. $68-69{ }^{\circ} \mathrm{C}, 455 \mathrm{mg}$, yield $67 \%,{ }^{1} \mathbf{H}$ NMR $\left(400 \mathrm{MHz}, \mathrm{CDCl}_{3}\right) \delta 9.45(\mathrm{~s}, 1 \mathrm{H}), 8.10(\mathrm{~s}, 1 \mathrm{H}), 7.90(\mathrm{~d}, J$ $=8.4 \mathrm{~Hz}, 1 \mathrm{H}), 7.57-7.49(\mathrm{~m}, 2 \mathrm{H}), 7.36(\mathrm{~d}, J=5.4 \mathrm{~Hz}, 1 \mathrm{H}) .{ }^{13} \mathbf{C}$ NMR $\left(101 \mathrm{MHz}, \mathrm{CDCl}_{3}\right) \delta 176.7,142.6,139.5,129.2,128.3,127.9,123.7$, 123.0, 115.1, 96.0, 88.4. HRMS (ESI) m/z $[\mathrm{M}+\mathrm{H}]^{+}$calcd for $\mathrm{C}_{11} \mathrm{H}_{7} \mathrm{OS}^{+}$: 187.0212, found: 187.0210 . 


\section{Supporting Information}

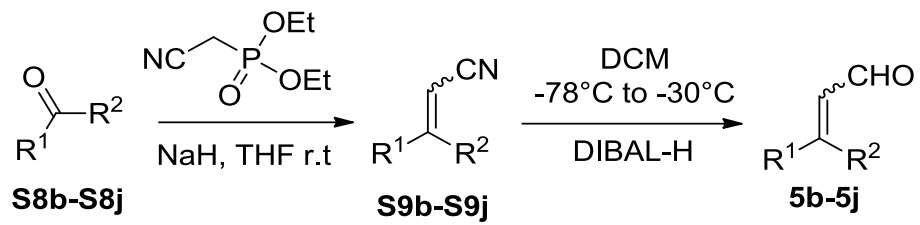

Scheme S5. Synthesis of enal via HWE reaction and reduction reaction

a) To a solution of diethyl cyanomethylphosphonate ( $1 \mathrm{~mL}, 6 \mathrm{mmol}, 1.2$ equiv) in dry THF $(10 \mathrm{~mL})$ was added $\mathrm{NaH}$ ( $50 \%$ in mineral oil, $0.36 \mathrm{~g}, 7.5 \mathrm{mmol}, 1.5$ equiv) in four portions in $2 \mathrm{~min}$ at $0{ }^{\circ} \mathrm{C}$. When there is no bubbles realesed, ketone or aldehyde substrate $\mathbf{S 8 b - S 8 j}$ (5 mmol, 1.0 equiv) was added to the reaction system slowly. The reaction mixture was allowed to react under vigorous stirring at room temperature for $3 \mathrm{~h}$. Water was added and the resulting solution was extracted with $\operatorname{DCM}(3 \times 10 \mathrm{~mL})$. The combined organic layers were dried over $\mathrm{Na}_{2} \mathrm{SO}_{4}$ and concentrated in vacuo. The crude residue was purified by flash chromatography affording the corresponding nitriles $\mathbf{S 9 b}$ S9j.

b) A dry flask was charged with compound $\mathbf{S 9 b - S 9 j ~ ( 4 ~ m m o l , ~} 1.0$ equiv) under $\mathrm{N}_{2}$ atmosphere, then dry DCM $(5 \mathrm{~mL})$ was injected to the system. The mixture was cooled to $-78{ }^{\circ} \mathrm{C}$ and DIBAL-H $(6$ $\mathrm{mL}, 1.0 \mathrm{M}$ in hexane, $6 \mathrm{mmol}, 1.5$ equiv) was added dropwise. The reaction temperature was allowed to warm up to $-30{ }^{\circ} \mathrm{C}$ within a period of $2 \mathrm{~h}$, and consumption of the starting material was monitored by TLC. When the reaction was complete, EA $(10 \mathrm{~mL})$ was added to the flask to consume excessive DIBAL-H. The solution was warmed to room temperature and further diluted with EA, followed by the addition of a saturated solution of Rochelle's salt. The suspension was stirred vigorously for $4 \mathrm{~h}$ to give a biphasic mixture. The aqueous layer was extracted with EA $(3 \times 10 \mathrm{~mL})$; the combined organic layers were dried over $\mathrm{Na}_{2} \mathrm{SO}_{4}$, filtered and concentrated in vacuo. The crude residue was purified by flash chromatography to give the corresponding aldehydes $\mathbf{5 b}-\mathbf{5 j}$.

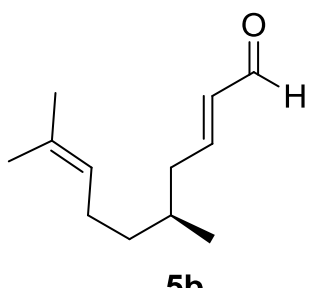

$5 b$<smiles>O=CC=C1CCC1</smiles>

5d
$(S, E)-5,9-D i m e t h y l d e c a-2,8-d i e n a l ~(5 b)^{[6 b, g]}$

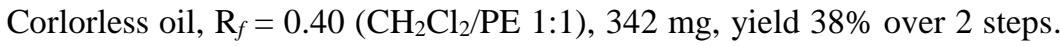
${ }^{1}$ H NMR $\left(400 \mathrm{MHz}, \mathrm{CDCl}_{3}\right) \delta 9.51(\mathrm{~d}, J=7.9 \mathrm{~Hz}, 1 \mathrm{H}), 6.82(\mathrm{dt}, J=15.1$, $7.4 \mathrm{~Hz}, 1 \mathrm{H}), 6.11(\mathrm{dd}, J=15.5,7.9 \mathrm{~Hz}, 1 \mathrm{H}), 5.07(\mathrm{dd}, J=10.0,4.1 \mathrm{~Hz}, 1 \mathrm{H})$, $2.46-2.28(\mathrm{~m}, 1 \mathrm{H}), 2.22(\mathrm{~d}, J=1.0 \mathrm{~Hz}, 1 \mathrm{H}), 2.10-1.92(\mathrm{~m}, 2 \mathrm{H}), 1.80-$ $1.64(\mathrm{~m}, 4 \mathrm{H}), 1.60(\mathrm{~s}, 3 \mathrm{H}), 1.46-1.30(\mathrm{~m}, 1 \mathrm{H}), 1.29-1.18(\mathrm{~m}, 1 \mathrm{H}), 0.93$ $(\mathrm{d}, J=6.7 \mathrm{~Hz}, 3 \mathrm{H}) .{ }^{13} \mathrm{C}$ NMR $\left(101 \mathrm{MHz}, \mathrm{CDCl}_{3}\right) \delta 194.0,157.7,134.2$, $131.7,124.2,40.1,36.7,32.1,25.7,25.4,19.5,17.6$.

\section{2-Cyclobutylideneacetaldehyde $(5 \mathrm{~d})^{[6 \mathrm{c}]}$}

Brown oil, $\mathrm{R}_{f}=0.40\left(\mathrm{CH}_{2} \mathrm{Cl}_{2} / \mathrm{PE} \mathrm{1:1)}, 154 \mathrm{mg}\right.$, yield $32 \%$ over 2 steps. ${ }^{1} \mathbf{H}$ NMR (400 MHz, $\mathrm{CDCl}_{3}$,) $\delta 9.61(\mathrm{~d}, J=8.2 \mathrm{~Hz}, 1 \mathrm{H}), 5.80$ (d, with small additional coupling, $J=8.2 \mathrm{~Hz}, 1 \mathrm{H}), 3.15$ (br t, $J=7.9 \mathrm{~Hz}, 2 \mathrm{H}$ ), 2.94 (br t, $J$ $=7.9 \mathrm{~Hz}, 2 \mathrm{H}), 2.12$ (q, $J=7.9 \mathrm{~Hz}, 2 \mathrm{H}) .{ }^{13} \mathbf{C} \mathbf{N M R}\left(101 \mathrm{MHz}, \mathrm{CDCl}_{3}\right)$ 190.1, $173.4,123.3,33.4,31.1,17.5$. 
<smiles>O=CC=C1CCCC1</smiles>

$5 e$<smiles>O=CC=C1CCCCC1</smiles>

$5 f$<smiles>O=CC=C1CCN(Cc2ccccc2)CC1</smiles><smiles>O=C/C=C1\CCC[Sn]CC1</smiles><smiles>O=CC=C1CCCCCCCCCC1</smiles>

$5 \mathbf{i}$
2-Cyclopentylideneacetaldehyde $(5 e)^{[6 d]}$

Corlorless oil, $\mathrm{R}_{f}=0.40\left(\mathrm{CH}_{2} \mathrm{Cl}_{2} / \mathrm{PE} \mathrm{1:1),} 220 \mathrm{mg}\right.$, yield $40 \%$ over 2 steps. ${ }^{1} \mathbf{H}$ NMR $\left(400 \mathrm{MHz}, \mathrm{CDCl}_{3}\right) \delta 9.85(\mathrm{~d}, J=8.0 \mathrm{~Hz}, 1 \mathrm{H}), 5.97(\mathrm{~d}, J=8.0 \mathrm{~Hz}$, $1 \mathrm{H}), 2.80(\mathrm{t}, J=7.2 \mathrm{~Hz}, 2 \mathrm{H}), 2.54(\mathrm{t}, J=7.2 \mathrm{~Hz}, 2 \mathrm{H}), 1.83(\mathrm{~m}, 2 \mathrm{H}), 1.72(\mathrm{~m}$, 2H). ${ }^{13} \mathbf{C}$ NMR $\left(101 \mathrm{MHz}, \mathrm{CDCl}_{3}\right) \delta 191.7,173.8,123.2,35.9,30.3,26.4$, 25.0 .

2-Cyclohexylideneacetaldehyde $(5 f)^{[6 d]}$

Corlorless oil, $\mathrm{R}_{f}=0.45\left(\mathrm{CH}_{2} \mathrm{Cl}_{2} / \mathrm{PE} \mathrm{1:1),} 217 \mathrm{mg}\right.$, yield $35 \%$ over 2 steps. ${ }^{1} \mathbf{H}$ NMR $\left(400 \mathrm{MHz}, \mathrm{CDCl}_{3}\right) \delta 10.01(\mathrm{~d}, J=8.2 \mathrm{~Hz}, 1 \mathrm{H}), 5.82(\mathrm{~d}, J=8.2 \mathrm{~Hz}$, $1 \mathrm{H}), 2.71(\mathrm{t}, J=6.4 \mathrm{~Hz}, 2 \mathrm{H}), 2.29(\mathrm{t}, J=6.4 \mathrm{~Hz}, 2 \mathrm{H}), 1.69(\mathrm{~m}, 6 \mathrm{H}) .{ }^{13} \mathrm{C}$ NMR $\left(101 \mathrm{MHz}, \mathrm{CDCl}_{3}\right) \delta 190.6,168.1,125.4,38.1,29.7,28.5,28.2,26.3$.

\section{2-(1-Benzylpiperidin-4-ylidene)acetaldehyde (5g)}

Yellow oil, $\mathrm{R}_{f}=0.20\left(\mathrm{CH}_{2} \mathrm{Cl}_{2} / \mathrm{PE} 1: 1\right), 312 \mathrm{mg}$, yield $29 \%$ over 2 steps. ${ }^{\mathbf{1}} \mathbf{H}$ NMR (400 MHz, $\left.\mathrm{CDCl}_{3}\right) \delta 9.99(\mathrm{~d}, J=8.1 \mathrm{~Hz}, 1 \mathrm{H}), 7.37-7.25(\mathrm{~m}, 5 \mathrm{H})$, $5.85(\mathrm{~d}, J=8.1 \mathrm{~Hz}, 1 \mathrm{H}), 3.55(\mathrm{~s}, 2 \mathrm{H}), 2.83(\mathrm{t}, J=5.6 \mathrm{~Hz}, 2 \mathrm{H}), 2.59(\mathrm{dd}, J=$ 7.8, $3.3 \mathrm{~Hz}, 4 \mathrm{H}), 2.42(\mathrm{t}, J=5.6 \mathrm{~Hz}, 2 \mathrm{H}) .{ }^{13} \mathbf{C ~ N M R}\left(101 \mathrm{MHz}, \mathrm{CDCl}_{3}\right) \delta$ 190.2, 164.0, 138.0, 128.9, 128.3, 127.2, 125.8, 62.3, 54.2, 53.9, 36.9, 29.1. HRMS (ESI) m/z [M+H] ${ }^{+}$calcd for $\mathrm{C}_{14} \mathrm{H}_{18} \mathrm{NO}^{+}: 216.1383$, found: 216.1388.

\section{2-Cycloheptylideneacetaldehyde $(5 \mathrm{~h})^{[6 \mathrm{e}]}$}

Yellow oil, $\mathrm{R}_{f}=0.45\left(\mathrm{CH}_{2} \mathrm{Cl}_{2} / \mathrm{PE} \mathrm{1:1),} 297 \mathrm{mg}\right.$, yield $43 \%$ over 2 steps. ${ }^{1} \mathbf{H}$ NMR $\left(400 \mathrm{MHz}, \mathrm{CDCl}_{3}\right) \delta 9.92(\mathrm{~d}, J=8.1 \mathrm{~Hz}, 1 \mathrm{H}), 5.78(\mathrm{~d}, J=8.1 \mathrm{~Hz}$, $1 \mathrm{H}), 2.84(\mathrm{t}, J=6.0 \mathrm{~Hz}, 2 \mathrm{H}), 2.45(\mathrm{t}, J=6.0 \mathrm{~Hz}, 2 \mathrm{H}), 1.77-1.63(\mathrm{~m}, 4 \mathrm{H})$, 1.55 - $1.46(\mathrm{~m}, 4 \mathrm{H}) .{ }^{13} \mathrm{C}$ NMR $\left(101 \mathrm{MHz}, \mathrm{CDCl}_{3}\right) \delta 191.1,170.2,127.6$, $38.9,30.5,29.3,28.6,27.4,27.3$.

\section{2-Cyclododecylideneacetaldehyde $(5 \mathbf{i})^{[6 \mathbf{6}]}$}

White solid, $\mathrm{R}_{f}=0.45\left(\mathrm{CH}_{2} \mathrm{Cl}_{2} / \mathrm{PE} \mathrm{1:1)}\right.$, M.p. $74-75{ }^{\circ} \mathrm{C}, 572 \mathrm{mg}$, yield $55 \%$ over 2 steps. ${ }^{1} \mathbf{H}$ NMR $\left(400 \mathrm{MHz}, \mathrm{CDCl}_{3}\right) \delta 10.01(\mathrm{~d}, J=8.2 \mathrm{~Hz}, 1 \mathrm{H}), 6.02$ $(\mathrm{d}, J=8.2 \mathrm{~Hz}, 1 \mathrm{H}), 2.63(\mathrm{t}, J=6.6 \mathrm{~Hz}, 2 \mathrm{H}), 2.31(\mathrm{t}, J=6.6 \mathrm{~Hz}, 2 \mathrm{H}), 1.40-$ $1.29(\mathrm{~m}, 18 \mathrm{H}) .{ }^{13} \mathrm{C}$ NMR $\left(101 \mathrm{MHz}, \mathrm{CDCl}_{3}\right) \delta 191.2,167.3,128.6,32.6$, $30.2,25.34,25.26,23.94,23.90,23.6,22.9,22.7,22.0$.

\section{2-(3-Methylcyclopentadecylidene)acetaldehyde (5j) $(E: Z=53: 47)$}

Colorless oil, $\mathrm{R}_{f}=0.50$ (PE/EA 20:1), $671 \mathrm{mg}$, yield $51 \%$ over 2 steps. ${ }^{1} \mathbf{H}$ NMR $\left(400 \mathrm{MHz}, \mathrm{CDCl}_{3}\right) \delta 9.96(\mathrm{dd}, J=8.1,5.8 \mathrm{~Hz}, 2 \mathrm{H}$, isomer a+b), 5.97 $(\mathrm{d}, J=8.2 \mathrm{~Hz}, 1 \mathrm{H}$, isomer a), $5.83(\mathrm{~d}, J=8.2 \mathrm{~Hz}, 1 \mathrm{H}$, isomer b), 2.68 (ddd, $J=13.3,9.7,6.2 \mathrm{~Hz}, 1 \mathrm{H}), 2.55-2.47(\mathrm{~m}, 1 \mathrm{H}), 2.50-2.31(\mathrm{~m}, 3 \mathrm{H}), 2.30-$ $2.21(\mathrm{~m}, 1 \mathrm{H}), 2.17-2.09(\mathrm{~m}, 1 \mathrm{H}), 1.88-1.66(\mathrm{~m}, 3 \mathrm{H}), 1.64-1.51(\mathrm{~m}, 2 \mathrm{H})$, 


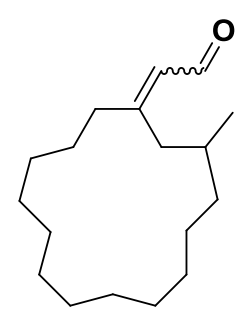

5j

$1.49-1.15(\mathrm{~m}, 42 \mathrm{H}$, isomer $\mathrm{a}+\mathrm{b}), 0.91(\mathrm{~d}, J=6.6 \mathrm{~Hz}, 3 \mathrm{H}$, isomer a), 0.84 $\left(\mathrm{d}, J=6.4 \mathrm{~Hz}, 3 \mathrm{H}\right.$, isomer b). ${ }^{13} \mathbf{C} \mathbf{N M R}\left(101 \mathrm{MHz}, \mathrm{CDCl}_{3}\right) \delta 191.2$ (isomer a), 191.0 (isomer b), 168.1 (isomer a), 167.9 (isomer b), 129.0 (isomer a), 128.85 (isomer b), 46.0 (isomer $\mathrm{a}+\mathrm{b}$ ), 38.3 (isomer a), 37.2 (isomer b), 36.0 (isomer a), 35.4 (isomer b), 30.7, 30.1, 29.8, 28.5, 27.7, 27.5, 26.9, 26.8 (isomer $\mathrm{a}+\mathrm{b}$ ), 26.7, 26.64, 26.57, 26.4, 26.34 (isomer $\mathrm{a}+\mathrm{b}$ ), 26.31, 26.3, 26.25, 26.2, 26.1, 25.1 (isomer a+b), 20.2 (isomer a), 20.1 (isomer b). HRMS (ESI) $\mathrm{m} / \mathrm{z}[\mathrm{M}+\mathrm{H}]^{+}$calcd for $\mathrm{C}_{18} \mathrm{H}_{33} \mathrm{O}^{+}:$265.2526, found: 265.2533.

\subsection{Synthesis of $12^{[7]}$}

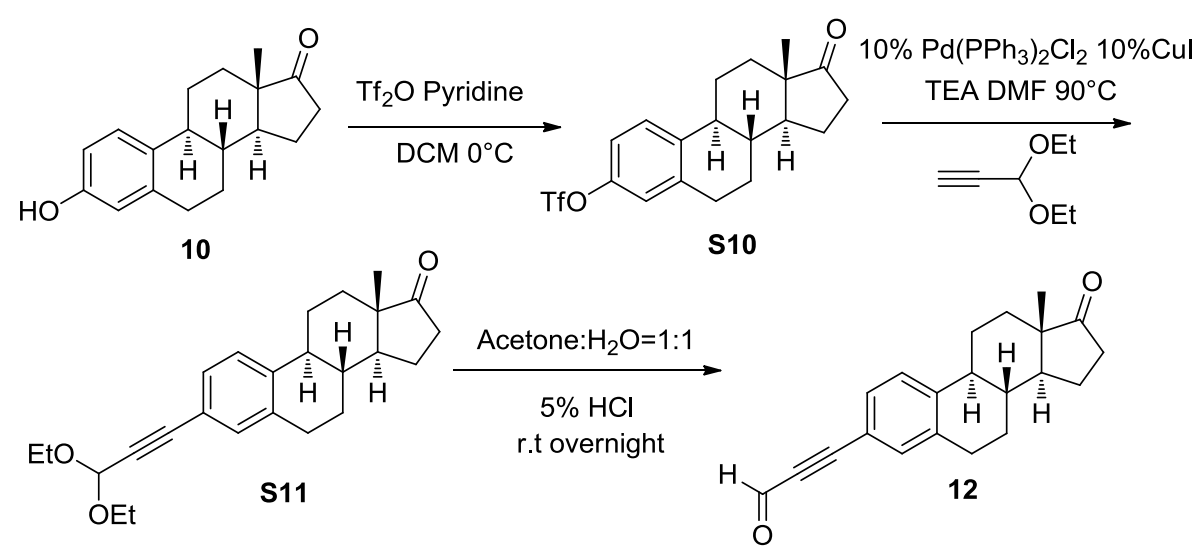

Scheme S6. Synthesis of 12 via Sonogashira coupling and acetal hydrolysis reaction

Estrone 10 (1.35 g, $5 \mathrm{mmol})$ and pyridine $(0.81 \mathrm{~mL}, 10.0 \mathrm{mmol})$ was sequentially dissolved in 20 $\mathrm{mL}$ of dry DCM, following by dropwise addition of $\mathrm{Tf}_{2} \mathrm{O}(1.0 \mathrm{~mL}, 6.0 \mathrm{mmol})$ in $10 \mathrm{~mL}$ of dry DCM at $0{ }^{\circ} \mathrm{C}$ via an addition funnel. After that, the mixture was warmed to rt, and stirred overnight. When the reaction completed, $10 \% \mathrm{HCl}$ was added to the solution to quench the reaction, and then the mixture was extracted with DCM. The organic layer was washed with saturated $\mathrm{NaHCO}_{3}$ and saturated brine, dried over anhydrous $\mathrm{Na}_{2} \mathrm{SO}_{4}$, filtered and concentrated in vacuo. Purification of the residue by column chromatography on silica gel gave the corresponding product $\mathbf{S 1 0}$ as a white solid in $91 \%$ yield $(1.83 \mathrm{~g})$.

\section{Synthesis of S11}

A mixture of $\mathbf{S 1 0}$ (1.81 g, $4.5 \mathrm{mmol})$, 3,3-diethoxyprop-1-yne (0.90 mL, $5.6 \mathrm{mmol})$, triethylamine (3.0 mL), and $\mathrm{Pd}\left(\mathrm{PPh}_{3}\right)_{2} \mathrm{Cl}_{2}(315 \mathrm{mg}, 0.45 \mathrm{mmol}), \mathrm{CuI}(86 \mathrm{mg}, 0.45 \mathrm{mmol})$ was dissolved in $15 \mathrm{~mL}$ of DMF and stirred at $90{ }^{\circ} \mathrm{C}$ for $4 \mathrm{~h}$ under nitrogen. The reaction mixture was diluted with water $(150 \mathrm{~mL})$ and extracted with EA $(3 \times 50 \mathrm{~mL})$, the combined organic layers were washed with brine for three times $(3 \times 30 \mathrm{~mL})$, dried by $\mathrm{Na}_{2} \mathrm{SO}_{4}$, and filtrated. The filtrate was evaporated. Chromatography of the residue on silica gel provided the corresponding product $\mathbf{S 1 1}$ with $90 \%$ yield $(1.56 \mathrm{~g})$.

\section{Synthesis of 12}

S11 (1.52 g, $4.01 \mathrm{mmol})$ was dissolved in mixed solvents $(20 \mathrm{~mL})$ of acetone and $\mathrm{H}_{2} \mathrm{O}(v: v=1: 1)$, 
then 5 equivalents of $5 \% \mathrm{HCl}(12 \mathrm{~mL}, 20 \mathrm{mmol})$ was added to the solution, and the mixture was allowed to react overnight at room temperature. Upon completion of the reaction, acetone was removed under reduced pressure, after that the aqueous layer was extracted with DCM $(3 \times 20 \mathrm{~mL})$, the combined organic layers were dried over anhydrous $\mathrm{Na}_{2} \mathrm{SO}_{4}$, and concentrated in vacuo. Purification of the residue by column chromatography gave 12 in $67 \%$ yield.

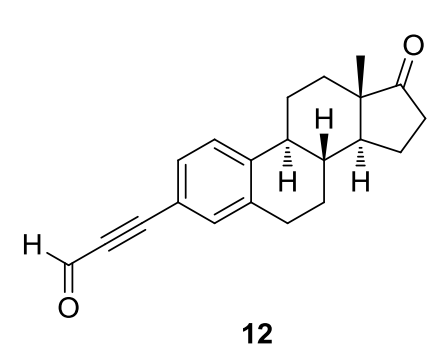

12

3-((8R,9S,13S,14S)-13-methyl-17-oxo-7,8,9,11,12,13,14,15,16,17decahydro- $6 H$-cyclopenta $[a]$ phenanthren-3-yl)propiolaldehyde (12)

White solid, $\mathrm{R}_{f}=0.30$ (PE/EA 20:1), M.p. $190-192{ }^{\circ} \mathrm{C}, 826 \mathrm{mg}$, overall $54 \%$ yield from 10. ${ }^{1} \mathbf{H}$ NMR $\left(400 \mathrm{MHz}, \mathrm{CDCl}_{3}\right) \delta 9.41(\mathrm{~s}$, $1 \mathrm{H}), 7.45-7.32(\mathrm{~m}, 3 \mathrm{H}), 3.12-2.80(\mathrm{~m}, 2 \mathrm{H}), 2.53(\mathrm{dd}, J=19,8 \mathrm{~Hz}$, 1H), $2.44-2.38(\mathrm{~m}, 1 \mathrm{H}), 2.31$ (td, $J=11,4 \mathrm{~Hz}, 1 \mathrm{H}), 2.17$ (dt, $J=$ $19,9 \mathrm{~Hz}, 1 \mathrm{H}), 2.11-2.03(\mathrm{~m}, 2 \mathrm{H}), 2.02-1.96(\mathrm{~m}, 1 \mathrm{H}), 1.73-1.39$ (m, 6H), 0.92 (s, 3H). ${ }^{13} \mathbf{C}$ NMR $\left(101 \mathrm{MHz}, \mathrm{CDCl}_{3}\right) \delta 220.4,176.8$, $143.9,137.3,133.8,130.6,125.8,116.6,95.9,88.4,50.5,47.8,44.6$, $37.7,35.8,31.5,29.0,26.1,25.4,21.5,13.8$. HRMS (ESI) $\mathrm{m} / \mathrm{z}$ $[\mathrm{M}+\mathrm{H}]^{+}$calcd for $\mathrm{C}_{21} \mathrm{H}_{23} \mathrm{O}_{2}{ }^{+}: 307.1693$, found: 307.1697 .

\subsection{Synthesis of 2-enal 15}

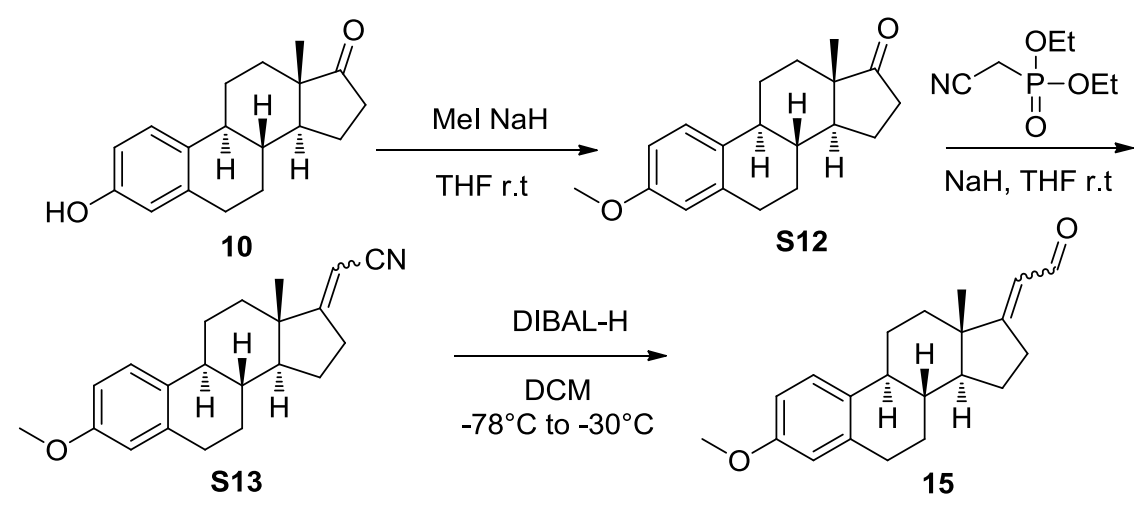

Scheme S7. Synthesis of $\mathbf{1 5}$ via Sonogashira coupling and reduction reaction

\section{Synthesis of $\mathrm{S12}{ }^{[8]}$}

A dry flask was charged with a solution of $\mathbf{1 0}(675 \mathrm{mg}, 2.5 \mathrm{mmol})$ and $\mathrm{NaH}(50 \%$ in mineral oil, $180 \mathrm{mg}, 3.75 \mathrm{mmol}, 1.5$ equiv) in anhydrous THF $(25 \mathrm{~mL})$ under nitrogen atmosphere. The mixture was stirred at $0{ }^{\circ} \mathrm{C}$ for $10 \mathrm{~min}$ and methyl iodide $(0.67 \mathrm{~mL}, 10 \mathrm{mmol})$ was added via a syringe. The resulting system was subsequently stirred for 12 hours at room temperature. After that another potion of methyl iodide $(0.67 \mathrm{~mL}, 10 \mathrm{mmol})$ was added to the mixture to react for another $12 \mathrm{~h}$. The resulting solution was then diluted with $\mathrm{EA}(50 \mathrm{~mL})$ and washed with a saturated aqueous solution of $\mathrm{NH}_{4} \mathrm{Cl}$. The organic layer was washed with water and brine, dried over $\mathrm{Na}_{2} \mathrm{SO}_{4}$, filtered and 


\section{Supporting Information}

evaporated under reduced pressure. The crude compound $\mathbf{S 1 2}$ was purified by flash chromatography in $89 \%$ yield $(632 \mathrm{mg})$ as a white solid.

S13 and $\mathbf{1 5}$ were synthesized according to general procedure for synthesis of en-2-als $\mathbf{5}$.

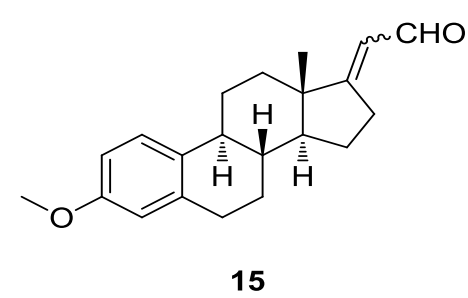

15

\section{2-((8S,9S,13S,14S)-3-Methoxy-13-methyl-}

7,8,9,11,12,13,15,16-octahydro-6H-

cyclopenta[a]phenanthren-17(14H)-ylidene)acetaldehyde (15)

$(E: Z=50: 50)$

White solid, $\mathrm{R}_{f}=0.40$ (PE/EA 10:1), M.p. $128-130{ }^{\circ} \mathrm{C}, 349 \mathrm{mg}$, overall $45 \%$ yield from 10. ${ }^{1} \mathbf{H}$ NMR $\left(400 \mathrm{MHz}, \mathrm{CDCl}_{3}\right) \delta 10.19$ (d, $J=8.7 \mathrm{~Hz}, 1 \mathrm{H}$, isomer a), $9.90(\mathrm{~d}, J=7.9 \mathrm{~Hz}, 1 \mathrm{H}$, isomer b), $7.21(\mathrm{dd}, J=8.5,3.6 \mathrm{~Hz}, 2 \mathrm{H}), 6.73(\mathrm{~d}, J=8.4 \mathrm{~Hz}, 2 \mathrm{H}$, isomer a), $6.65(\mathrm{~d}, J=2.6 \mathrm{~Hz}, 2 \mathrm{H}$, isomer b), $5.86(\mathrm{~d}, J=8.7 \mathrm{~Hz}, 1 \mathrm{H}$, isomer a), $5.81(\mathrm{~d}, J=7.9 \mathrm{~Hz}, 1 \mathrm{H}$, isomer b), $3.78(\mathrm{~s}, 6 \mathrm{H}), 3.13-2.81(\mathrm{~m}$, $6 \mathrm{H}), 2.71(\mathrm{~d}, J=8.7 \mathrm{~Hz}, 1 \mathrm{H}), 2.57-2.32(\mathrm{~m}, 4 \mathrm{H}), 2.32-2.14(\mathrm{~m}$, $2 \mathrm{H}), 2.13-1.84(\mathrm{~m}, 5 \mathrm{H}), 1.73-1.28(\mathrm{~m}, 12 \mathrm{H}), 1.11(\mathrm{~s}, 3 \mathrm{H}$, isomer a), 0.91 (s, 3H, isomer b). ${ }^{13} \mathbf{C}$ NMR (101 MHz, $\left.\mathrm{CDCl}_{3}\right) \delta$ 192.3 (isomer a), 190.7 (isomer b), 178.0 (isomer a), 178.8 (isomer b), 157.59 (isomer a), 157.55 (isomer b), 137.8 (isomer a), 137.7 (isomer b), 132.1 (isomer a), 131.9 (isomer b), 126.3 (isomer $\mathrm{a}+\mathrm{b}$ ), 124.1 (isomer a), 119.4 (isomer b), 113.8 (isomer a+b), 111.6 (isomer a), 111.5 (isomer b), 55.2 (isomer a+b), 54.3 (isomer a), 52.2 (isomer b), 47.5 (isomer a), 46.8 (isomer b), 43.9 (isomer a), 43.2 (isomer b), 38.9, 38.5, 38.4, 34.9, 33.4, 29.72 (isomer a), 29.70 (isomer b), 27.7, 27.6, 27.4, 27.0, 26.4, 24.0 (isomer a), 23.7 (isomer b), 19.1 (isomer a), 18.2 (isomer b). HRMS (ESI) $\mathrm{m} / \mathrm{z}[\mathrm{M}+\mathrm{H}]^{+}$calcd for $\mathrm{C}_{21} \mathrm{H}_{27} \mathrm{O}_{2}{ }^{+}: 311.2006$, found: 311.2014 .

\section{Experimental procedure for the reactions of thiodiazoles 2 and ynals 1.}

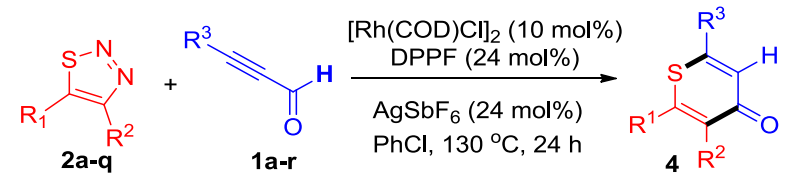

Scheme S8. Reactions of thiodiazoles 2 and ynals $\mathbf{1}$

\subsection{General procedure:}

A dry reaction tube equipped with a magnetic stirring bar was charged with thiadiazole (2a-q, 0.200 mmol), $[\mathrm{Rh}(\mathrm{COD}) \mathrm{Cl}]_{2}(10 \mathrm{mg}, 0.020 \mathrm{mmol})$, DPPF $(27 \mathrm{mg}, 0.048 \mathrm{mmol})$ and $\mathrm{AgSbF}_{6}(16.5 \mathrm{mg}$, 
$0.048 \mathrm{mmol})$. The tube was sealed immediately and protected with nitrogen by evacuation-backfill operations for three times, followed by slow addition of a solution of ynals substrate (1a-r, 0.400 mmol) in fresh distilled $\mathrm{PhCl}(1.5 \mathrm{~mL})$ via a syringe. The mixtrure was heated at $130{ }^{\circ} \mathrm{C}$ for 24 hours. When the reaction was finished, the solvent was evaporated under reduced pressure, and the crude mixture was subjected to flash chromatography on silica gel affording the corresponding product 4.<smiles>CCOC(=O)c1c(-c2ccccc2)sc(-c2ccccc2)cc1=O</smiles>

Ethyl 4-oxo-2,6-diphenyl-4H-thiopyran-3-carboxylate (4aa) Brown oil, $\mathbf{R}_{f}=0.40$ (PE/EA 3:1), $41 \mathrm{mg}$, yield 61\%. ${ }^{1} \mathbf{H}$ NMR (400 $\left.\mathrm{MHz}, \mathrm{CDCl}_{3}\right) \delta 7.63-7.60(\mathrm{~m}, 2 \mathrm{H}), 7.55-7.44(\mathrm{~m}, 8 \mathrm{H}), 7.31(\mathrm{~s}, 1 \mathrm{H}), 4.15$ (q, $J=7.1 \mathrm{~Hz}, 2 \mathrm{H}), 1.03(\mathrm{t}, J=7.1 \mathrm{~Hz}, 3 \mathrm{H}) .{ }^{13} \mathbf{C} \mathbf{~ N M R}\left(101 \mathrm{MHz}, \mathrm{CDCl}_{3}\right)$ $\delta$ 178.6, 165.4, 153.0, 152.1, 135.5, 134.6, 134.4, 131.0, 130.6, 129.5, 128.9, 128.2, 127.4, 126.8, 61.7, 13.8. HRMS (ESI) $\mathrm{m} / \mathrm{z}[\mathrm{M}+\mathrm{H}]^{+}$calcd for $\mathrm{C}_{20} \mathrm{H}_{17} \mathrm{O}_{3} \mathrm{~S}^{+}: 337.0893$, found: 337.0892 .<smiles>CCOC(=O)c1c(-c2ccccc2)sc(-c2ccc(C)cc2)cc1=O</smiles>

$4 a b$<smiles>CCOC(=O)c1c(-c2ccccc2)sc(-c2ccc(OC)cc2)cc1=O</smiles>

4 ac
Ethyl 6-(4-(methylthio)phenyl)-4-oxo-2-phenyl-4H-thiopyran-3carboxylate (4ab)

Brown oil, $\mathrm{R}_{f}=0.39$ (PE/EA 3:1), $48 \mathrm{mg}$, yield 63\%. ${ }^{1} \mathbf{H}$ NMR $(400 \mathrm{MHz}$, $\left.\mathrm{CDCl}_{3}\right) \delta 7.56-7.50(\mathrm{~m}, 4 \mathrm{H}), 7.50-7.42(\mathrm{~m}, 3 \mathrm{H}), 7.31(\mathrm{~d}, J=8.5 \mathrm{~Hz}$, 2H), 7.29 (s, 1H), $4.14(\mathrm{q}, J=7.1 \mathrm{~Hz}, 2 \mathrm{H}), 2.52(\mathrm{~s}, 3 \mathrm{H}), 1.01(\mathrm{t}, J=7.1 \mathrm{~Hz}$, 3H). ${ }^{13} \mathrm{C}$ NMR $\left(101 \mathrm{MHz}, \mathrm{CDCl}_{3}\right) \delta 178.6,165.3,152.4,151.8,143.3$, 134.5, 134.2, 131.5, 130.5, 128.9, 128.1, 126.9, 126.4, 126.3, 61.7, 15.0, 13.7. HRMS (ESI) $\mathrm{m} / \mathrm{z}[\mathrm{M}+\mathrm{H}]^{+}$calcd for $\mathrm{C}_{21} \mathrm{H}_{19} \mathrm{O}_{3} \mathrm{~S}_{2}{ }^{+}: 383.0770$, found: 383.0778.

Ethyl 6-(4-methoxyphenyl)-4-oxo-2-phenyl-4H-thiopyran-3carboxylate (4ac)

Brown oil, $\mathrm{R}_{f}=0.39$ (PE/EA 3:1), $42 \mathrm{mg}$, yield 58\%. ${ }^{\mathbf{1}} \mathbf{H}$ NMR (400 MHz, $\left.\mathrm{CDCl}_{3}\right) \delta 7.61-7.40(\mathrm{~m}, 7 \mathrm{H}), 7.26(\mathrm{~s}, 1 \mathrm{H}), 7.00(\mathrm{~d}, J=8.8 \mathrm{~Hz}, 2 \mathrm{H}), 4.14$ (q, $J=7.1 \mathrm{~Hz}, 2 \mathrm{H}), 3.87(\mathrm{~s}, 3 \mathrm{H}), 1.02(\mathrm{t}, J=7.1 \mathrm{~Hz}, 3 \mathrm{H}) .{ }^{13} \mathbf{C}$ NMR $(101$ $\left.\mathrm{MHz}, \mathrm{CDCl}_{3}\right) \delta 178.7,165.5,162.0,152.7,151.8,134.6,134.1,130.5$, $128.9,128.2,127.7,126.0,114.8,61.7,55.5,13.7$. HRMS (ESI) $\mathrm{m} / \mathrm{z}$ $[\mathrm{M}+\mathrm{Na}]^{+}$calcd for $\mathrm{C}_{21} \mathrm{H}_{18} \mathrm{NaO}_{4} \mathrm{~S}^{+}: 389.0818$, found: 389.0812 . 
<smiles>CCOC(=O)c1c(-c2ccccc2)sc(-c2ccccc2OC)cc1=O</smiles>

4ad

Ethyl 6-(2-methoxyphenyl)-4-oxo-2-phenyl-4H-thiopyran-3carboxylate (4ad)

Brown oil, $\mathrm{R}_{f}=0.21(\mathrm{PE} / \mathrm{EA} 3: 1), 44 \mathrm{mg}$, yield 61\%. ${ }^{1} \mathbf{H}$ NMR $(400 \mathrm{MHz}$, $\left.\mathrm{CDCl}_{3}\right) \delta 7.54(\mathrm{dd}, J=7.7,1.6 \mathrm{~Hz}, 2 \mathrm{H}), 7.47(\mathrm{qd}, J=6.8,3.1 \mathrm{~Hz}, 4 \mathrm{H}), 7.39$ $(\mathrm{dd}, J=7.6,1.5 \mathrm{~Hz}, 1 \mathrm{H}), 7.26(\mathrm{~s}, 1 \mathrm{H}), 7.05(\mathrm{ddd}, J=13.7,10.3,4.5 \mathrm{~Hz}$, $2 \mathrm{H}), 4.16(\mathrm{q}, J=7.1 \mathrm{~Hz}, 2 \mathrm{H}), 3.87(\mathrm{~s}, 3 \mathrm{H}), 1.04(\mathrm{t}, J=7.1 \mathrm{~Hz}, 3 \mathrm{H}) .{ }^{13} \mathrm{C}$ NMR $\left(101 \mathrm{MHz}, \mathrm{CDCl}_{3}\right) \delta 178.5,165.6,156.4,153.1,150.3,134.7,133.9$, 131.9, 130.6, 130.3, 130.1, 128.8, 128.2, 124.2, 121.0, 111.6, 61.6, 55.7, 13.7. HRMS (ESI) $\mathrm{m} / \mathrm{z}[\mathrm{M}+\mathrm{H}]^{+}$calcd for $\mathrm{C}_{21} \mathrm{H}_{19} \mathrm{O}_{4} \mathrm{~S}^{+}: 367.0999$, found: 367.1007.<smiles>CCOC(=O)c1c(-c2ccccc2)sc(-c2ccc(C)cc2)cc1=O</smiles>

4ae<smiles>CCOC(=O)c1c(-c2ccccc2)sc(-c2ccc(F)cc2)cc1=O</smiles>

4af<smiles>CCOC(=O)c1c(-c2ccccc2)sc(-c2cccc(Cl)c2)cc1=O</smiles>

4ag
Ethyl 6-(4-fluorophenyl)-4-oxo-2-phenyl-4H-thiopyran-3-carboxylate (4af)

Brown oil, $\mathrm{R}_{f}=0.47$ (PE/EA 3:1), $46 \mathrm{mg}$, yield 65\%. ${ }^{\mathbf{1}} \mathbf{H}$ NMR (400 MHz, $\left.\mathrm{CDCl}_{3}\right) \delta 7.57-7.50(\mathrm{~m}, 2 \mathrm{H}), 7.48-7.36(\mathrm{~m}, 5 \mathrm{H}), 7.19(\mathrm{~s}, 1 \mathrm{H}), 7.12(\mathrm{t}, J$ $=8.5 \mathrm{~Hz}, 2 \mathrm{H}), 4.07(\mathrm{q}, J=7.1 \mathrm{~Hz}, 2 \mathrm{H}), 0.95(\mathrm{t}, J=7.1 \mathrm{~Hz}, 3 \mathrm{H}) .{ }^{13} \mathbf{C} \mathbf{N M R}$ $\left(101 \mathrm{MHz}, \mathrm{CDCl}_{3}\right) \delta 178.5,165.2,164.4(\mathrm{~d}, J=253.5 \mathrm{~Hz}), 151.9,151.8$, 134.4, 134.3, $131.6(\mathrm{~d}, J=3.4 \mathrm{~Hz}), 130.6,128.9,128.8(\mathrm{~d}, J=8.7 \mathrm{~Hz})$, $128.1,127.3,116.7(\mathrm{~d}, J=22.1 \mathrm{~Hz}), 61.8,13.7$. HRMS (ESI) $\mathrm{m} / \mathrm{z}[\mathrm{M}+\mathrm{H}]^{+}$ calcd for $\mathrm{C}_{20} \mathrm{H}_{16} \mathrm{FO}_{3} \mathrm{~S}^{+}: 355.0799$, found: 355.0806 .

Ethyl 6-(3-chlorophenyl)-4-oxo-2-phenyl-4H-thiopyran-3-carboxylate (4ag)

Brown oil, $\mathrm{R}_{f}=0.39\left(\mathrm{PE} / \mathrm{EA} \mathrm{3:1)}, 38 \mathrm{mg}\right.$, yield 51\%. ${ }^{1} \mathbf{H}$ NMR $(400 \mathrm{MHz}$, $\left.\mathrm{CDCl}_{3}\right) \delta 7.60(\mathrm{t}, J=1.7 \mathrm{~Hz}, 1 \mathrm{H}), 7.56-7.38(\mathrm{~m}, 8 \mathrm{H}), 7.29(\mathrm{~s}, 1 \mathrm{H}), 4.15$ $(\mathrm{q}, J=7.1 \mathrm{~Hz}, 2 \mathrm{H}), 1.03(\mathrm{t}, J=7.1 \mathrm{~Hz}, 3 \mathrm{H}) .{ }^{13} \mathbf{C ~ N M R}\left(101 \mathrm{MHz}, \mathrm{CDCl}_{3}\right)$ $\delta 178.4,165.1,151.9,151.3,137.1,135.6,134.5,134.3,131.0,130.72$, 130.68, 129.0, 128.2, 127.9, 126.9, 125.0, 61.8, 13.7. HRMS (ESI) m/z $[\mathrm{M}+\mathrm{H}]^{+}$calcd for $\mathrm{C}_{20} \mathrm{H}_{16} \mathrm{ClO}_{3} \mathrm{~S}^{+}: 371.0503$, found: 371.0510 . 
<smiles>CCC(C)(C)c1c(-c2ccccc2)sc(-c2ccccc2Cl)cc1=O</smiles><smiles>CCOC(=O)c1c(-c2ccccc2)sc(-c2ccc(Br)cc2)cc1=O</smiles>

4ai<smiles>CCOC(=O)c1c(-c2ccccc2)sc(-c2ccc(I)cc2)cc1=O</smiles>

4aj

Ethyl 6-(2-chlorophenyl)-4-oxo-2-phenyl-4H-thiopyran-3-carboxylate (4ah)

Brown oil, $\mathrm{R}_{f}=0.39$ (PE/EA 3:1), $52 \mathrm{mg}$, yield 70\%. ${ }^{1} \mathbf{H}$ NMR $(400 \mathrm{MHz}$, $\left.\mathrm{CDCl}_{3}\right) \delta 7.56-7.36(\mathrm{~m}, 9 \mathrm{H}), 7.12(\mathrm{~s}, 1 \mathrm{H}), 4.16(\mathrm{q}, J=7.1 \mathrm{~Hz}, 2 \mathrm{H}), 1.04$ $(\mathrm{t}, J=7.1 \mathrm{~Hz}, 3 \mathrm{H}) .{ }^{13} \mathrm{C}$ NMR $\left(101 \mathrm{MHz}, \mathrm{CDCl}_{3}\right) \delta 178.1,165.3,152.8$, $150.5,134.33,134.28,134.0,132.6,131.4,131.3,130.7,130.6,130.57$, 128.9, 128.1, 127.3, 61.8, 13.8. HRMS (ESI) $\mathrm{m} / \mathrm{z}[\mathrm{M}+\mathrm{H}]^{+}$calcd for $\mathrm{C}_{20} \mathrm{H}_{16} \mathrm{ClO}_{3} \mathrm{~S}^{+}$: 371.0503 , found: 371.0510 .

Ethyl 6-(4-bromophenyl)-4-oxo-2-phenyl-4H-thiopyran-3-carboxylate (4ai)

Brown oil, $\mathrm{R}_{f}=0.50$ (PE/EA 3:1), $50 \mathrm{mg}$, yield 60\%. ${ }^{1} \mathbf{H}$ NMR $(400 \mathrm{MHz}$, $\left.\mathrm{CDCl}_{3}\right) \delta 7.63(\mathrm{~d}, J=8.5 \mathrm{~Hz}, 2 \mathrm{H}), 7.53-7.46(\mathrm{~m}, 7 \mathrm{H}), 7.27(\mathrm{~s}, 1 \mathrm{H}), 4.14$ $(\mathrm{q}, J=7.1 \mathrm{~Hz}, 2 \mathrm{H}), 1.02(\mathrm{t}, J=7.1 \mathrm{~Hz}, 3 \mathrm{H}) .{ }^{13} \mathbf{C ~ N M R}\left(101 \mathrm{MHz}, \mathrm{CDCl}_{3}\right)$ $\delta$ 178.4, 165.2, 151.9, 151.7, 134.4, 134.30, 134.27, 132.7, 130.6, 128.9, 128.2, 128.1, 127.4, 125.7, 61.8, 13.7. HRMS (ESI) m/z $[\mathrm{M}+\mathrm{Na}]^{+}$calcd for $\mathrm{C}_{20} \mathrm{H}_{15} \mathrm{BrNaO}_{3} \mathrm{~S}^{+}$: 436.9817 , found: 436.9816 .

Ethyl 6-(4-iodophenyl)-4-oxo-2-phenyl-4H-thiopyran-3-carboxylate (4aj)

Brown oil, $\mathrm{R}_{f}=0.27$ (PE/EA 3:1), $16 \mathrm{mg}$, yield 17\%. ${ }^{1} \mathbf{H}$ NMR $(400 \mathrm{MHz}$, $\left.\mathrm{CDCl}_{3}\right) \delta 7.84(\mathrm{~d}, J=8.5 \mathrm{~Hz}, 2 \mathrm{H}), 7.56-7.41(\mathrm{~m}, 5 \mathrm{H}), 7.34(\mathrm{~d}, J=8.4 \mathrm{~Hz}$, $2 \mathrm{H}), 7.28(\mathrm{~s}, 1 \mathrm{H}), 4.14(\mathrm{q}, J=7.1 \mathrm{~Hz}, 2 \mathrm{H}), 1.02(\mathrm{t}, J=7.1 \mathrm{~Hz}, 3 \mathrm{H}) .{ }^{13} \mathrm{C}$ NMR $\left(101 \mathrm{MHz}, \mathrm{CDCl}_{3}\right) \delta 178.4,165.2,151.8,138.7,134.9,134.4$, 134.37, 130.7, 128.9, 128.26, 128.17, 127.4, 97.6, 61.8, 13.7. HRMS (ESI) $\mathrm{m} / \mathrm{z}[\mathrm{M}+\mathrm{H}]^{+}$calcd for $\mathrm{C}_{20} \mathrm{H}_{16} \mathrm{IO}_{3} \mathrm{~S}^{+}: 462.9859$, found: 462.9864 .<smiles>CCOC(=O)c1c(-c2ccccc2)sc(-c2ccc(C#N)cc2)cc1=O</smiles>

Ethyl 6-(4-cyanophenyl)-4-oxo-2-phenyl-4H-thiopyran-3-carboxylate (4ak)

Brown oil, $\mathrm{R}_{f}=0.25$ (PE/EA 3:1), $48 \mathrm{mg}$, yield 66\%. ${ }^{1} \mathbf{H}$ NMR $(400 \mathrm{MHz}$, $\left.\mathrm{CDCl}_{3}\right) \delta 7.80(\mathrm{~d}, J=8.5 \mathrm{~Hz}, 2 \mathrm{H}), 7.73(\mathrm{~d}, J=8.5 \mathrm{~Hz}, 2 \mathrm{H}), 7.55-7.41(\mathrm{~m}$, $5 \mathrm{H}), 7.32(\mathrm{~s}, 1 \mathrm{H}), 4.15(\mathrm{q}, J=7.1 \mathrm{~Hz}, 2 \mathrm{H}), 1.03(\mathrm{t}, J=7.1 \mathrm{~Hz}, 3 \mathrm{H}) .{ }^{13} \mathrm{C}$ NMR (101 MHz, $\left.\mathrm{CDCl}_{3}\right) \delta 178.2,164.9,150.6,139.5,135.0,134.6,134.1$, 133.2, 130.8, 129.0, 128.7, 128.1, 127.5, 117.7, 114.7, 61.9, 13.7. HRMS (ESI) $\mathrm{m} / \mathrm{z}[\mathrm{M}+\mathrm{H}]^{+}$calcd for $\mathrm{C}_{21} \mathrm{H}_{16} \mathrm{NO}_{3} \mathrm{~S}^{+}: 362.0845$, found: 362.0854 . 
<smiles>CCOC(=O)c1c(-c2ccccc2)sc(-c2ccc([N+](=O)[O-])cc2)cc1=O</smiles>

4al<smiles>CCOC(=O)c1c(-c2ccccc2)sc(-c2ccc(C(C)=O)cc2)cc1=O</smiles>

4am

Ethyl 6-(4-nitrophenyl)-4-oxo-2-phenyl-4H-thiopyran-3-carboxylate (4al)

Brown oil, $\mathrm{R}_{f}=0.31(\mathrm{PE} / \mathrm{EA} 3: 1), 38 \mathrm{mg}$, yield 50\%. ${ }^{1} \mathbf{H}$ NMR $(400 \mathrm{MHz}$, $\left.\mathrm{CDCl}_{3}\right) \delta 8.37(\mathrm{~d}, J=8.8 \mathrm{~Hz}, 2 \mathrm{H}), 7.80(\mathrm{~d}, J=8.8 \mathrm{~Hz}, 2 \mathrm{H}), 7.55-7.46(\mathrm{~m}$, 5H), $7.35(\mathrm{~s}, 1 \mathrm{H}), 4.15(\mathrm{~d}, J=7.1 \mathrm{~Hz}, 2 \mathrm{H}), 1.03(\mathrm{t}, J=7.1 \mathrm{~Hz}, 3 \mathrm{H}) .{ }^{13} \mathbf{C}$ NMR (101 MHz, $\left.\mathrm{CDCl}_{3}\right) \delta 178.2,164.9,150.2,149.2,141.3,140.5,134.7$, 134.1, 130.9, 129.1, 129.0, 128.2, 128.0, 124.7, 62.0, 13.8. HRMS (ESI) $\mathrm{m} / \mathrm{z}[\mathrm{M}+\mathrm{H}]^{+}$calcd for $\mathrm{C}_{20} \mathrm{H}_{16} \mathrm{NO}_{5} \mathrm{~S}^{+}: 382.0744$, found: 382.0744 .

Ethyl 6-(4-acetylphenyl)-4-oxo-2-phenyl-4H-thiopyran-3-carboxylate (4am)

Brown oil, $\mathrm{R}_{f}=0.16$ (PE/EA 3:1), $34 \mathrm{mg}$, yield 45\%. ${ }^{1} \mathbf{H}$ NMR $(400 \mathrm{MHz}$, $\left.\mathrm{CDCl}_{3}\right) \delta 8.10(\mathrm{~d}, J=8.3 \mathrm{~Hz}, 2 \mathrm{H}), 7.74(\mathrm{~d}, J=8.3 \mathrm{~Hz}, 2 \mathrm{H}), 7.62-7.44(\mathrm{~m}$, $5 \mathrm{H}), 7.37$ (s, 1H), 4.17 (q, J=7.1 Hz, 2H), $2.68(\mathrm{~s}, 3 \mathrm{H}), 1.05(\mathrm{t}, J=7.1 \mathrm{~Hz}$, 3H). ${ }^{13} \mathbf{C}$ NMR $\left(101 \mathrm{MHz}, \mathrm{CDCl}_{3}\right) \delta 197.0,178.3,165.1,152.0,151.5$, $139.5,138.7,134.5,134.3,130.7,129.3,129.0,128.2,128.1,127.1,61.8$, 26.7, 13.7. HRMS (ESI) $\mathrm{m} / \mathrm{z}[\mathrm{M}+\mathrm{H}]^{+}$calcd for $\mathrm{C}_{22} \mathrm{H}_{19} \mathrm{O}_{4} \mathrm{~S}^{+}: 379.0999$, found: 379.0996 .<smiles>CCOC(=O)c1c(-c2ccccc2)sc(-c2cccc3ccccc23)cc1=O</smiles>

4an<smiles>CCOC(=O)c1c(-c2ccccc2)sc(-c2ccc3sccc3c2)cc1=O</smiles>

Ethyl 6-(naphthalen-1-yl)-4-oxo-2-phenyl-4H-thiopyran-3carboxylate (4an)

Brown oil, $\mathrm{R}_{f}=0.50$ (PE/EA 3:1), $54 \mathrm{mg}$, yield 70\%. ${ }^{1} \mathbf{H}$ NMR (400 MHz, $\left.\mathrm{CDCl}_{3}\right) \delta 8.05(\mathrm{dd}, J=6.2,3.5 \mathrm{~Hz}, 1 \mathrm{H}), 7.98(\mathrm{dd}, J=6.0,3.4 \mathrm{~Hz}, 1 \mathrm{H}), 7.93$ $(\mathrm{dd}, J=6.1,3.4 \mathrm{~Hz}, 1 \mathrm{H}), 7.65-7.51(\mathrm{~m}, 6 \mathrm{H}), 7.53-7.37(\mathrm{~m}, 3 \mathrm{H}), 7.25(\mathrm{~s}$, 1H), 4.19 (q, $J=7.1 \mathrm{~Hz}, 2 \mathrm{H}), 1.07$ (t, $J=7.1 \mathrm{~Hz}, 3 \mathrm{H}) .{ }^{13} \mathbf{C}$ NMR $(101 \mathrm{MHz}$, $\left.\mathrm{CDCl}_{3}\right) \delta 178.1,165.5,153.1,152.2,134.5,134.4,133.7,132.8,131.3$, 130.8, 130.6, 130.4, 128.9, 128.6, 128.1, 127.5, 127.3, 126.8, 125.0, 124.6, 61.8, 13.8. HRMS (ESI) $\mathrm{m} / \mathrm{z}[\mathrm{M}+\mathrm{H}]^{+}$calcd for $\mathrm{C}_{24} \mathrm{H}_{19} \mathrm{O}_{3} \mathrm{~S}^{+}: 387.1049$, found: 387.1056 .

Ethyl 6-(benzo[b]thiophen-5-yl)-4-oxo-2-phenyl-4H-thiopyran-3carboxylate (4ao)

Brown oil, $\mathrm{R}_{f}=0.29$ (PE/EA 3:1), $37 \mathrm{mg}$, yield 48\%. ${ }^{1} \mathbf{H}$ NMR $(400 \mathrm{MHz}$, $\left.\mathrm{CDCl}_{3}\right) \delta 8.09(\mathrm{~s}, 1 \mathrm{H}), 7.99(\mathrm{~d}, J=8.5 \mathrm{~Hz}, 1 \mathrm{H}), 7.59-7.56(\mathrm{~m}, 3 \mathrm{H}), 7.52$ $-7.44(\mathrm{~m}, 3 \mathrm{H}), 7.42(\mathrm{~d}, J=5.4 \mathrm{~Hz}, 1 \mathrm{H}), 7.38(\mathrm{~s}, 1 \mathrm{H}), 7.26(\mathrm{~s}, 1 \mathrm{H}), 4.16(\mathrm{q}$, $J=7.1 \mathrm{~Hz}, 2 \mathrm{H}), 1.04(\mathrm{t}, J=7.1 \mathrm{~Hz}, 3 \mathrm{H}) .{ }^{13} \mathbf{C ~ N M R}\left(101 \mathrm{MHz}, \mathrm{CDCl}_{3}\right) \delta$ 178.6, 165.4, 153.3, 152.1, 142.2, 140.1, 134.6, 134.3, 131.8, 130.6, 128.9, 128.6, 128.2, 127.4, 124.1, 123.6, 122.4, 122.1, 61.8, 13.8. HRMS (ESI) 
$\mathrm{m} / \mathrm{z}[\mathrm{M}+\mathrm{H}]^{+}$calcd for $\mathrm{C}_{22} \mathrm{H}_{17} \mathrm{O}_{3} \mathrm{~S}_{2}^{+}$: 393.0614, found: 393.0621 .<smiles>CCOC(=O)c1c(-c2ccccc2)sc(Br)cc1=O</smiles>

4ap<smiles>CCCc1cc(=O)c(C(=O)OCC)c(-c2ccccc2)s1</smiles>

$4 a q$<smiles>CCOC(=O)c1c(-c2ccccc2)sc(C(C)(C)C)cc1=O</smiles>

4ar<smiles>CCOC(=O)c1c(-c2ccccc2)sc(C2CC2)cc1=O</smiles><smiles>CCOC(=O)c1c(-c2ccccc2)sc(C2CCCC2)cc1=O</smiles>

Ethyl 6-butyl-4-oxo-2-phenyl-4H-thiopyran-3-carboxylate (4ap)

Brown oil, $\mathrm{R}_{f}=0.30$ (PE/EA 3:1), $44 \mathrm{mg}$, yield 70\%. ${ }^{1} \mathbf{H}$ NMR $(400 \mathrm{MHz}$, $\left.\mathrm{CDCl}_{3}\right) \delta 7.52-7.36(\mathrm{~m}, 5 \mathrm{H}), 6.93(\mathrm{~s}, 1 \mathrm{H}), 4.11(\mathrm{q}, J=7.1 \mathrm{~Hz}, 2 \mathrm{H}), 2.64$ (t, $J=7.6 \mathrm{~Hz}, 2 \mathrm{H}), 1.63$ (quint, $J=7.1 \mathrm{~Hz}, 2 \mathrm{H}), 1.40$ (sext, $J=7.1 \mathrm{~Hz}, 2 \mathrm{H}$ ), 0.99 (t, $J=7.1 \mathrm{~Hz}, 3 \mathrm{H}), 0.94(\mathrm{t}, J=7.3 \mathrm{~Hz}, 3 \mathrm{H}) .{ }^{13} \mathbf{C}$ NMR $(101 \mathrm{MHz}$, $\left.\mathrm{CDCl}_{3}\right) \delta 178.4,165.4,156.0,151.9,134.6,134.2,130.4,128.8,128.04$, 128.00, 61.6, 36.3, 31.7, 21.8, 13.7, 13.6. HRMS (ESI) m/z $[\mathrm{M}+\mathrm{H}]^{+}$calcd for $\mathrm{C}_{18} \mathrm{H}_{21} \mathrm{O}_{3} \mathrm{~S}^{+}: 317.1206$, found: 317.1212 .

Ethyl 4-oxo-2-phenyl-6-propyl-4H-thiopyran-3-carboxylate (4aq) Brown oil, $\mathrm{R}_{f}=0.29$ (PE/EA 3:1), $40 \mathrm{mg}$, yield 68\%. ${ }^{1} \mathbf{H}$ NMR $(400 \mathrm{MHz}$, $\left.\mathrm{CDCl}_{3}\right) \delta 7.52-7.36(\mathrm{~m}, 5 \mathrm{H}), 6.94(\mathrm{~s}, 1 \mathrm{H}), 4.12(\mathrm{q}, J=7.1 \mathrm{~Hz}, 2 \mathrm{H}), 2.62$ (t, $J=7.5 \mathrm{~Hz}, 2 \mathrm{H}), 1.40$ (sext, $J=7.1 \mathrm{~Hz}, 2 \mathrm{H}), 1.01$ (dt, $J=7.3,2.0 \mathrm{~Hz}$, 6H). ${ }^{13} \mathrm{C}$ NMR $\left(101 \mathrm{MHz}, \mathrm{CDCl}_{3}\right) \delta 178.4,165.5,155.7,151.9,134.6$, 134.2, 130.4, 128.8, 128.2, 128.1, 61.6, 38.5, 23.0, 13.7, 13.3. HRMS (ESI) $\mathrm{m} / \mathrm{z}[\mathrm{M}+\mathrm{H}]^{+}$calcd for $\mathrm{C}_{17} \mathrm{H}_{19} \mathrm{O}_{3} \mathrm{~S}^{+}: 303.1049$, found: 303.1052 .

Ethyl 6-(tert-butyl)-4-oxo-2-phenyl-4H-thiopyran-3-carboxylate (4ar) Brown oil, $\mathrm{R}_{f}=0.33$ (PE/EA 3:1), $24 \mathrm{mg}$, yield 39\%. ${ }^{1} \mathbf{H}$ NMR $(400 \mathrm{MHz}$, $\left.\mathrm{CDCl}_{3}\right) \delta 7.59-7.37(\mathrm{~m}, 5 \mathrm{H}), 7.08(\mathrm{~s}, 1 \mathrm{H}), 4.12(\mathrm{q}, J=7.1 \mathrm{~Hz}, 2 \mathrm{H}), 1.39$ $(\mathrm{s}, 9 \mathrm{H}), 1.00(\mathrm{t}, J=7.1 \mathrm{~Hz}, 3 \mathrm{H}) .{ }^{13} \mathbf{C}$ NMR $\left(101 \mathrm{MHz}, \mathrm{CDCl}_{3}\right) \delta 178.9$, 165.7, 165.5, 151.9, 134.9, 133.6, 130.4, 128.8, 128.2, 125.7, 61.6, 38.5, 30.4, 13.8. HRMS (ESI) $\mathrm{m} / \mathrm{z}[\mathrm{M}+\mathrm{H}]^{+}$calcd for $\mathrm{C}_{18} \mathrm{H}_{21} \mathrm{O}_{3} \mathrm{~S}^{+}: 317.1206$ found: 317.1215 .

Ethyl 6-cyclopropyl-4-oxo-2-phenyl-4H-thiopyran-3-carboxylate (4as)

Brown oil, $\mathrm{R}_{f}=0.23$ (PE/EA 3:1), $24 \mathrm{mg}$, yield 40\%. ${ }^{1} \mathbf{H}$ NMR (400 MHz, $\left.\mathrm{CDCl}_{3}\right) \delta 7.53-7.35(\mathrm{~m}, 5 \mathrm{H}), 6.82(\mathrm{~s}, 1 \mathrm{H}), 4.10(\mathrm{q}, J=7.1 \mathrm{~Hz}, 2 \mathrm{H}), 1.96$ $-1.87(\mathrm{~m}, 1 \mathrm{H}), 1.14(\mathrm{dt}, J=6.9,4.9 \mathrm{~Hz}, 2 \mathrm{H}), 0.99(\mathrm{t}, J=7.1 \mathrm{~Hz}, 3 \mathrm{H}), 0.92$ -0.79 (m, 2H). ${ }^{13} \mathbf{C}$ NMR $\left(101 \mathrm{MHz}, \mathrm{CDCl}_{3}\right) \delta 178.2,165.4,158.4,151.3$, 134.6, 134.3, 130.4 128.8, 128.0, 125.0, 61.6, 16.9, 13.7, 9.9. HRMS (ESI) $\mathrm{m} / \mathrm{z}[\mathrm{M}+\mathrm{H}]^{+}$calcd for $\mathrm{C}_{17} \mathrm{H}_{17} \mathrm{O}_{3} \mathrm{~S}^{+}: 301.0893$, found: 301.0894 .

Ethyl 6-cyclopentyl-4-oxo-2-phenyl-4H-thiopyran-3-carboxylate (4at) Brown oil, $\mathrm{R}_{f}=0.25$ (PE/EA 3:1), $52 \mathrm{mg}$, yield 80\%. ${ }^{1} \mathbf{H}$ NMR $(400 \mathrm{MHz}$, $\left.\mathrm{CDCl}_{3}\right) \delta 7.58-7.36(\mathrm{~m}, 5 \mathrm{H}), 6.97(\mathrm{~s}, 1 \mathrm{H}), 4.10(\mathrm{q}, J=7.1 \mathrm{~Hz}, 2 \mathrm{H}), 3.00$ $(\mathrm{p}, J=8.2 \mathrm{~Hz}, 1 \mathrm{H}), 2.14-1.78(\mathrm{~m}, 2 \mathrm{H}), 1.86-1.78(\mathrm{~m}, 2 \mathrm{H}), 1.75-1.67$ $(\mathrm{m}, 4 \mathrm{H}), 0.99(\mathrm{t}, J=7.1 \mathrm{~Hz}, 3 \mathrm{H}) .{ }^{13} \mathbf{C}$ NMR $\left(101 \mathrm{MHz}, \mathrm{CDCl}_{3}\right) \delta 178.5$, 165.4, 160.5, 151.8, 134.7, 134.2, 130.3, 128.7, 128.0, 126.4, 61.5, 46.7, 33.4, 25.3, 13.7. HRMS (ESI) $\mathrm{m} / \mathrm{z}[\mathrm{M}+\mathrm{H}]^{+}$calcd for $\mathrm{C}_{19} \mathrm{H}_{21} \mathrm{O}_{3} \mathrm{~S}^{+}$: 329.1206, found: 329.1209 . 
<smiles>CCOC(=O)c1c(-c2ccccc2)sc(C2CCCCC2)cc1=O</smiles><smiles>CCOC(=O)c1c(-c2ccc3c(c2)OCO3)sc(-c2ccccc2)cc1=O</smiles><smiles>CCOC(=O)c1c(-c2ccc(OC)cc2)sc(-c2ccccc2)cc1=O</smiles><smiles>CCOC(=O)c1c(-c2ccc(C)cc2)sc(-c2ccccc2)cc1=O</smiles>

Ethyl 6-cyclohexyl-4-oxo-2-phenyl-4H-thiopyran-3-carboxylate (4au) Brown oil, $\mathrm{R}_{f}=0.25$ (PE/EA 3:1), $56 \mathrm{mg}$, yield 82\%. ${ }^{1} \mathbf{H}$ NMR $(400 \mathrm{MHz}$, $\left.\mathrm{CDCl}_{3}\right) \delta 7.58-7.36(\mathrm{~m}, 5 \mathrm{H}), 6.93(\mathrm{~s}, 1 \mathrm{H}), 4.09(\mathrm{q}, J=7.1 \mathrm{~Hz}, 2 \mathrm{H}), 2.49$ (tt, $J=11.7,2.8 \mathrm{~Hz}, 1 \mathrm{H}), 1.96(\mathrm{~d}, J=11.8 \mathrm{~Hz}, 2 \mathrm{H}), 1.85(\mathrm{~d}, J=12.8 \mathrm{~Hz}$, $2 \mathrm{H}), 1.73(\mathrm{~d}, J=12.7 \mathrm{~Hz}, 1 \mathrm{H}), 1.53-1.40(\mathrm{~m}, 2 \mathrm{H}), 1.40-1.27(\mathrm{~m}, 2 \mathrm{H})$, $1.27-1.16(\mathrm{~m}, 1 \mathrm{H}), 0.98(\mathrm{t}, J=7.1 \mathrm{~Hz}, 3 \mathrm{H}) .{ }^{13} \mathbf{C ~ N M R}\left(101 \mathrm{MHz}, \mathrm{CDCl}_{3}\right)$ $\delta 178.5,165.4,161.4,151.7,134.7,134.2,130.3,128.7,128.0,126.3,61.5$, 45.7, 33.2, 26.1, 25.4, 13.6. HRMS (ESI) $\mathrm{m} / \mathrm{z}[\mathrm{M}+\mathrm{H}]^{+}$calcd for $\mathrm{C}_{20} \mathrm{H}_{23} \mathrm{O}_{3} \mathrm{~S}^{+}$: 343.1362, found: 343.1370 .

Ethyl 2-(benzo[d][1,3]dioxol-5-yl)-4-oxo-6-phenyl-4H-thiopyran-3carboxylate (4ba) Brown oil, $\mathrm{R}_{f}=0.29$ (PE/EA 3:1), $43 \mathrm{mg}$, yield 57\%. ${ }^{1} \mathbf{H}$ NMR $(400 \mathrm{MHz}$, $\left.\mathrm{CDCl}_{3}\right) \delta 7.60(\mathrm{dd}, J=7.6,1.8 \mathrm{~Hz}, 2 \mathrm{H}), 7.52-7.47(\mathrm{~m}, 3 \mathrm{H}), 7.28(\mathrm{~s}, 1 \mathrm{H})$, $7.05(\mathrm{dd}, J=8.0,1.8 \mathrm{~Hz}, 1 \mathrm{H}), 7.02(\mathrm{~d}, J=1.7 \mathrm{~Hz}, 1 \mathrm{H}), 6.87(\mathrm{~d}, J=8.0 \mathrm{~Hz}$, 1H), 6.04 (s, 2H), 4.23 (q, $J=7.1 \mathrm{~Hz}, 2 \mathrm{H}), 1.15(\mathrm{t}, J=7.1 \mathrm{~Hz}, 3 \mathrm{H}) .{ }^{13} \mathrm{C}$ NMR $\left(101 \mathrm{MHz}, \mathrm{CDCl}_{3}\right) \delta 178.7,165.6,152.9,151.6,149.6,148.1,135.4$, 134.1, 131.0, 129.4, 128.1, 127.2, 126.8, 122.7, 108.7, 108.5, 101.2, 61.8, 13.9. HRMS (ESI) $\mathrm{m} / \mathrm{z}[\mathrm{M}+\mathrm{H}]^{+}$calcd for $\mathrm{C}_{21} \mathrm{H}_{17} \mathrm{O}_{5} \mathrm{~S}^{+}: 381.0791$, found: 381.0798 .

Ethyl 2-(4-methoxyphenyl)-4-oxo-6-phenyl-4H-thiopyran-3carboxylate (4ca)

Brown oil, $\mathrm{R}_{f}=0.29$ (PE/EA 3:1), $37 \mathrm{mg}$, yield 50\%. ${ }^{1} \mathbf{H}$ NMR $(400 \mathrm{MHz}$, $\left.\mathrm{CDCl}_{3}\right) \delta 7.61(\mathrm{dd}, J=7.5,1.9 \mathrm{~Hz}, 2 \mathrm{H}), 7.54-7.42(\mathrm{~m}, 5 \mathrm{H}), 7.29(\mathrm{~s}, 1 \mathrm{H})$, $6.96(\mathrm{~d}, J=8.8 \mathrm{~Hz}, 2 \mathrm{H}), 4.19(\mathrm{q}, J=7.1 \mathrm{~Hz}, 2 \mathrm{H}), 3.86(\mathrm{~s}, 3 \mathrm{H}), 1.10(\mathrm{t}, J=$ $7.1 \mathrm{~Hz}, 3 \mathrm{H}) .{ }^{13} \mathrm{C}$ NMR $\left(101 \mathrm{MHz}, \mathrm{CDCl}_{3}\right) \delta 178.8,165.7,161.4,153.0$, 152.0, 136.6, 135.5, 134.0, 130.9, 129.7, 129.4, 127.2, 126.8, 114.3, 61.7, 55.4, 13.8. HRMS (ESI) $\mathrm{m} / \mathrm{z}[\mathrm{M}+\mathrm{H}]^{+}$calcd for $\mathrm{C}_{21} \mathrm{H}_{19} \mathrm{O}_{4} \mathrm{~S}^{+}: 367.0999$, found: 367.1006 .

Ethyl 4-oxo-6-phenyl-2-( $p$-tolyl)-4H-thiopyran-3-carboxylate (4da) Brown oil, $\mathrm{R}_{f}=0.43$ (PE/EA 3:1), $40 \mathrm{mg}$, yield 58\%. ${ }^{1} \mathbf{H}$ NMR $(400 \mathrm{MHz}$, $\left.\mathrm{CDCl}_{3}\right) \delta 7.61(\mathrm{dd}, J=7.6 \mathrm{~Hz}, 1.9 \mathrm{~Hz}, 2 \mathrm{H}), 7.53-7.45(\mathrm{~m}, 3 \mathrm{H}), 7.43(\mathrm{~d}, J$ $=8.2 \mathrm{~Hz}, 2 \mathrm{H}), 7.29(\mathrm{~s}, 1 \mathrm{H}), 7.26(\mathrm{~d}, J=7.6 \mathrm{~Hz}, 2 \mathrm{H}), 4.17(\mathrm{q}, J=7.1 \mathrm{~Hz}$, $2 \mathrm{H}), 2.41(\mathrm{~s}, 3 \mathrm{H}), 1.08(\mathrm{t}, J=7.1 \mathrm{~Hz}, 3 \mathrm{H}) .{ }^{13} \mathbf{C} \mathbf{N M R}\left(101 \mathrm{MHz}, \mathrm{CDCl}_{3}\right) \delta$ 178.7, 165.6, 153.0, 152.2, 141.0, 135.5, 134.1, 131.7, 130.9, 129.6, 129.4, 128.0, 127.3, 126.8, 61.7, 21.4, 13.9. HRMS (ESI) $\mathrm{m} / \mathrm{z}[\mathrm{M}+\mathrm{H}]^{+}$calcd for $\mathrm{C}_{21} \mathrm{H}_{19} \mathrm{O}_{3} \mathrm{~S}^{+}:$351.1049, found: 351.1057 . 


\section{Supporting Information}<smiles>CCOCc1c(-c2ccc(F)cc2)sc(-c2ccccc2)cc1=O</smiles><smiles>CCOCc1c(-c2ccc(Cl)cc2)sc(-c2ccccc2)cc1=O</smiles><smiles>CCOCc1c(-c2ccc(Br)cc2)sc(-c2ccccc2)cc1=O</smiles><smiles>CCOC(=O)c1c(-c2ccc(I)cc2)sc(-c2ccccc2)cc1=O</smiles>

Ethyl 2-(4-fluorophenyl)-4-oxo-6-phenyl-4H-thiopyran-3-carboxylate (4ea)

Brown oil, $\mathrm{R}_{f}=0.40(\mathrm{PE} / \mathrm{EA} 3: 1), 48 \mathrm{mg}$, yield 68\%. ${ }^{1} \mathbf{H}$ NMR $(400 \mathrm{MHz}$, $\left.\mathrm{CDCl}_{3}\right) \delta 7.74(\mathrm{~d}, J=8.3 \mathrm{~Hz}, 2 \mathrm{H}), 7.67(\mathrm{~d}, J=8.2 \mathrm{~Hz}, 2 \mathrm{H}), 7.61(\mathrm{dd}, J=$ 7.8, $1.7 \mathrm{~Hz}, 2 \mathrm{H}), 7.52-7.48(\mathrm{~m}, 3 \mathrm{H}), 7.33(\mathrm{~s}, 1 \mathrm{H}), 4.16(\mathrm{q}, J=7.1 \mathrm{~Hz}, 2 \mathrm{H})$, $1.05(\mathrm{t}, J=7.1 \mathrm{~Hz}, 3 \mathrm{H}) .{ }^{13} \mathbf{C}$ NMR $\left(101 \mathrm{MHz}, \mathrm{CDCl}_{3}\right) \delta 178.4,165.2,163.9$ $(\mathrm{d}, J=252.0 \mathrm{~Hz}), 152.9,150.7,135.2,134.6,131.0,130.5$ (d, $J=3.5 \mathrm{~Hz})$, $130.3(\mathrm{~d}, J=8.7 \mathrm{~Hz}), 129.4,127.3,126.7,116.1$ (d, $J=22.0 \mathrm{~Hz}), 61.8$, 13.8. HRMS (ESI) $\mathrm{m} / \mathrm{z}[\mathrm{M}+\mathrm{H}]^{+}$calcd for $\mathrm{C}_{20} \mathrm{H}_{16} \mathrm{FO}_{3} \mathrm{~S}^{+}: 355.0799$, found: 355.0800 .

Ethyl 2-(4-chlorophenyl)-4-oxo-6-phenyl-4H-thiopyran-3-carboxylate (4fa)

Brown oil, $\mathrm{R}_{f}=0.43(\mathrm{PE} / \mathrm{EA} 3: 1), 47 \mathrm{mg}$, yield 64\%. ${ }^{1} \mathbf{H}$ NMR $(400 \mathrm{MHz}$, $\left.\mathrm{CDCl}_{3}\right) \delta 7.62-7.57(\mathrm{~m}, 2 \mathrm{H}), 7.54-7.40(\mathrm{~m}, 7 \mathrm{H}), 7.30(\mathrm{~s}, 1 \mathrm{H}), 4.17$ (q, $J$ $=7.1 \mathrm{~Hz}, 2 \mathrm{H}), 1.09(\mathrm{t}, J=7.1 \mathrm{~Hz}, 3 \mathrm{H}) \cdot{ }^{13} \mathbf{C ~ N M R}\left(101 \mathrm{MHz}, \mathrm{CDCl}_{3}\right) \delta$ $178.4,165.2,152.9,150.5,137.0,135.2,134.5,132.8,131.1,129.5,129.4$, 129.2, 127.4, 126.7, 61.9, 13.8. HRMS (ESI) $\mathrm{m} / \mathrm{z}[\mathrm{M}+\mathrm{H}]^{+}$calcd for $\mathrm{C}_{20} \mathrm{H}_{16} \mathrm{ClO}_{3} \mathrm{~S}^{+}:$371.0503, found: 371.0511 .

Ethyl 2-(4-bromophenyl)-4-oxo-6-phenyl-4H-thiopyran-3-carboxylate (4ga)

Brown oil, $\mathrm{R}_{f}=0.43$ (PE/EA 3:1), $58 \mathrm{mg}$, yield 70\%. ${ }^{1} \mathbf{H}$ NMR $(400 \mathrm{MHz}$, $\left.\mathrm{CDCl}_{3}\right) \delta 7.62-7.59(\mathrm{~m}, 4 \mathrm{H}), 7.52-7.50(\mathrm{~m}, 3 \mathrm{H}), 7.41(\mathrm{~d}, J=8.5 \mathrm{~Hz}$, $2 \mathrm{H}), 7.31(\mathrm{~s}, 1 \mathrm{H}), 4.18(\mathrm{q}, J=7.1 \mathrm{~Hz}, 2 \mathrm{H}), 1.10(\mathrm{t}, J=7.1 \mathrm{~Hz}, 3 \mathrm{H}) .{ }^{13} \mathrm{C}$ NMR $\left(101 \mathrm{MHz}, \mathrm{CDCl}_{3}\right) \delta 178.4,165.2,152.9,150.5,135.3,134.5,133.3$, 132.2, 131.1, 129.8, 129.5, 127.4, 126.8, 125.2, 61.9, 13.8. HRMS (ESI) $\mathrm{m} / \mathrm{z}[\mathrm{M}+\mathrm{Na}]^{+}$calcd for $\mathrm{C}_{20} \mathrm{H}_{15} \mathrm{BrNaO}_{3} \mathrm{~S}^{+}:$436.9817, found: 436.9808 .

Ethyl 2-(4-iodophenyl)-4-oxo-6-phenyl-4H-thiopyran-3-carboxylate (4ha)

Brown oil, $\mathrm{R}_{f}=0.43\left(\mathrm{PE} / \mathrm{EA} \mathrm{3:1),} 66 \mathrm{mg}\right.$, yield 72\%. ${ }^{1} \mathbf{H}$ NMR $(400 \mathrm{MHz}$, $\left.\mathrm{CDCl}_{3}\right) \delta 7.81(\mathrm{~d}, J=8.3 \mathrm{~Hz}, 2 \mathrm{H}), 7.70-7.55(\mathrm{~m}, 2 \mathrm{H}), 7.57-7.45(\mathrm{~m}$, $3 \mathrm{H}), 7.30(\mathrm{~s}, 1 \mathrm{H}), 7.27(\mathrm{~d}, J=8.4 \mathrm{~Hz}, 2 \mathrm{H}), 4.18(\mathrm{q}, J=7.1 \mathrm{~Hz}, 2 \mathrm{H}), 1.10$ $(\mathrm{t}, J=7.1 \mathrm{~Hz}, 3 \mathrm{H}) .{ }^{13} \mathrm{C}$ NMR $\left(101 \mathrm{MHz}, \mathrm{CDCl}_{3}\right) \delta 178.4,161.1,152.9$, 150.7, 138.2, 135.3, 135.2, 133.9, 131.1, 129.8, 129.5, 127.4, 126.8, 97.2, 61.9, 13.8. HRMS (ESI) $\mathrm{m} / \mathrm{z}[\mathrm{M}+\mathrm{H}]^{+}$calcd for $\mathrm{C}_{20} \mathrm{H}_{16} \mathrm{IO}_{3} \mathrm{~S}^{+}: 462.9859$, found: 462.9858 . 
<smiles>CCOc1c(-c2ccc(Cl)c(Cl)c2)sc(-c2ccccc2)cc1=O</smiles><smiles>CCOCc1c(-c2ccc(C(F)(F)F)cc2)sc(-c2ccccc2)cc1=O</smiles><smiles>CCOCc1c(-c2ccc(C#N)cc2)sc(-c2ccccc2)cc1=O</smiles><smiles>CCOc1c(-c2ccc3ccccc3c2)sc(-c2ccccc2)cc1=O</smiles>

Ethyl 2-(3,4-dichlorophenyl)-4-oxo-6-phenyl-4H-thiopyran-3carboxylate (4ia)

Brown oil, $\mathrm{R}_{f}=0.43$ (PE/EA 3:1), $46 \mathrm{mg}$, yield 57\%. ${ }^{1} \mathbf{H}$ NMR (400 MHz, $\left.\mathrm{CDCl}_{3}\right) \delta 7.66(\mathrm{~d}, J=2.0 \mathrm{~Hz}, 1 \mathrm{H}), 7.63-7.58(\mathrm{~m}, 2 \mathrm{H}), 7.53(\mathrm{dd}, J=15.8$, $8.0 \mathrm{~Hz}, 4 \mathrm{H}), 7.39(\mathrm{dd}, J=8.3,2.0 \mathrm{~Hz}, 1 \mathrm{H}), 7.30(\mathrm{~s}, 1 \mathrm{H}), 4.22$ (q, $J=7.1$ $\mathrm{Hz}, 2 \mathrm{H}), 1.15(\mathrm{t}, J=7.1 \mathrm{~Hz}, 3 \mathrm{H}) .{ }^{13} \mathbf{C} \mathbf{~ N M R}\left(101 \mathrm{MHz}, \mathrm{CDCl}_{3}\right) \delta 178.3$, 164.9, 152.8, 148.8, 135.3, 135.1, 134.8, 134.1, 133.5, 131.2, 131.0, 130.1, 129.5, 127.5, 127.4 126.8, 62.1, 13.9. HRMS (ESI) $\mathrm{m} / \mathrm{z}[\mathrm{M}+\mathrm{H}]^{+}$calcd for $\mathrm{C}_{20} \mathrm{H}_{15} \mathrm{Cl}_{2} \mathrm{O}_{3} \mathrm{~S}^{+}:$405.0113, found: 405.0121 .

Ethyl 4-oxo-6-phenyl-2-(4-(trifluoromethyl)phenyl)-4H-thiopyran-3carboxylate (4ja)

Brown oil, $\mathrm{R}_{f}=0.43$ (PE/EA 3:1), $42 \mathrm{mg}$, yield 52\%. ${ }^{1} \mathbf{H}$ NMR (400 MHz, $\left.\mathrm{CDCl}_{3}\right) \delta 7.61(\mathrm{dd}, J=7.7,1.7 \mathrm{~Hz}, 2 \mathrm{H}), 7.56-7.46(\mathrm{~m}, 5 \mathrm{H}), 7.31(\mathrm{~s}, 1 \mathrm{H})$, $7.16(\mathrm{t}, J=8.6 \mathrm{~Hz}, 2 \mathrm{H}), 4.17(\mathrm{~d}, J=7.1 \mathrm{~Hz}, 2 \mathrm{H}), 1.08(\mathrm{t}, J=7.1 \mathrm{~Hz}, 3 \mathrm{H})$. ${ }^{13} \mathbf{C}$ NMR $\left(101 \mathrm{MHz}, \mathrm{CDCl}_{3}\right) \delta 178.2,164.9,152.8,150.0,137.9,135.2$, $134.9,134.8,131.2,129.5,128.8,127.5,126.8,126.5,125.9$ (q, $J=3.7$ $\mathrm{Hz}$ ), 62.0, 13.7. HRMS (ESI) $\mathrm{m} / \mathrm{z}[\mathrm{M}+\mathrm{H}]^{+}$calcd for $\mathrm{C}_{21} \mathrm{H}_{16} \mathrm{~F}_{3} \mathrm{O}_{3} \mathrm{~S}^{+}$: 405.0767, found: 405.0771 .

Ethyl 2-(4-cyanophenyl)-4-oxo-6-phenyl-4H-thiopyran-3-carboxylate (4ka)

Brown oil, $\mathrm{R}_{f}=0.26$ (PE/EA 3:1), $56 \mathrm{mg}$, yield 78\%. ${ }^{1} \mathbf{H}$ NMR (400 MHz, $\left.\mathrm{CDCl}_{3}\right) \delta 7.77(\mathrm{~d}, J=8.5 \mathrm{~Hz}, 2 \mathrm{H}), 7.66(\mathrm{~d}, J=8.5 \mathrm{~Hz}, 2 \mathrm{H}), 7.62-7.57(\mathrm{~m}$, 2H), $7.55-7.46(\mathrm{~m}, 3 \mathrm{H}), 7.31$ (s, 1H), 4.15 (q, $J=7.1 \mathrm{~Hz}, 2 \mathrm{H}), 1.07$ (t, $J$ $=7.1 \mathrm{~Hz}, 3 \mathrm{H}) .{ }^{13} \mathbf{C} \mathbf{N M R}\left(101 \mathrm{MHz}, \mathrm{CDCl}_{3}\right) \delta 178.0,164.7,152.7,149.3$, 138.7, 135.0, 134.9, 132.6, 131.3, 129.5, 129.1, 127.5, 126.7, 117.7, 114.5, 62.0, 13.8. HRMS (ESI) $\mathrm{m} / \mathrm{z}[\mathrm{M}+\mathrm{H}]^{+}$calcd for $\mathrm{C}_{21} \mathrm{H}_{16} \mathrm{NO}_{3} \mathrm{~S}^{+}: 362.0845$, found: 362.0839 .

Ethyl 2-(naphthalen-2-yl)-4-oxo-6-phenyl-4H-thiopyran-3carboxylate (4la)

Brown oil, $\mathrm{R}_{f}=0.40$ (PE/EA 3:1), $34 \mathrm{mg}$, yield 43\%. ${ }^{1} \mathbf{H}$ NMR (400 MHz, $\left.\mathrm{CDCl}_{3}\right) \delta 8.06(\mathrm{~s}, 1 \mathrm{H}), 7.96-7.86(\mathrm{~m}, 3 \mathrm{H}), 7.68-7.54(\mathrm{~m}, 5 \mathrm{H}), 7.52(\mathrm{t}, J$ $=5.8 \mathrm{~Hz}, 3 \mathrm{H}), 7.34(\mathrm{~s}, 1 \mathrm{H}), 4.13(\mathrm{q}, J=7.1 \mathrm{~Hz}, 2 \mathrm{H}), 0.98(\mathrm{t}, J=7.1 \mathrm{~Hz}$, 3H). ${ }^{13} \mathrm{C}$ NMR $\left(101 \mathrm{MHz}, \mathrm{CDCl}_{3}\right) \delta 178.6,165.5,153.1,152.1,135.4$, 134.5, 133.8, 132.7, 131.8, 131.0, 129.4, 128.9, 128.5, 128.3, 127.8, 127.7, 127.4, 127.2, 126.8, 124.9, 61.8, 13.8. HRMS (ESI) m/z [M+Na $]^{+}$calcd for $\mathrm{C}_{24} \mathrm{H}_{18} \mathrm{NaO}_{3} \mathrm{~S}^{+}: 409.0869$, found: 409.0876 . 


\section{Supporting Information}<smiles>CCOc1c(-c2ccco2)sc(-c2ccccc2)cc1=O</smiles><smiles>CCOc1c(-c2cccs2)sc(-c2ccccc2)cc1=O</smiles><smiles>COC(=O)c1c(C)sc(-c2ccccc2)cc1=O</smiles><smiles>O=c1cc(-c2ccccc2)sc(-c2ccccc2)c1-c1ccccc1</smiles>

4pa<smiles>CCOC(=O)c1c(C2CCC2)sc(C2CCCCC2)cc1=O</smiles>

Ethyl 2-(furan-2-yl)-4-oxo-6-phenyl-4H-thiopyran-3-carboxylate (4ma)

Brown oil, $\mathrm{R}_{f}=0.26$ (PE/EA 3:1), $44 \mathrm{mg}$, yield 68\%. ${ }^{1} \mathbf{H}$ NMR $(400 \mathrm{MHz}$, $\left.\mathrm{CDCl}_{3}\right) \delta 7.64-7.58(\mathrm{~m}, 3 \mathrm{H}), 7.57-7.44(\mathrm{~m}, 3 \mathrm{H}), 7.20(\mathrm{~s}, 1 \mathrm{H}), 6.94(\mathrm{~d}, J$ $=3.6 \mathrm{~Hz}, 1 \mathrm{H}), 6.57(\mathrm{dd}, J=3.6,1.8 \mathrm{~Hz}, 1 \mathrm{H}), 4.44(\mathrm{q}, J=7.1 \mathrm{~Hz}, 2 \mathrm{H}), 1.36$ (t, $J=7.1 \mathrm{~Hz}, 3 \mathrm{H}) .{ }^{13} \mathbf{C}$ NMR $\left(101 \mathrm{MHz}, \mathrm{CDCl}_{3}\right) \delta 178.9,168.7,166.1$, 151.8, 145.6, 138.6, 135.5, 131.1, 129.4, 126.84, 126.76, 120.3, 113.6, 112.8, 62.2, 14.1. HRMS (ESI) $\mathrm{m} / \mathrm{z}[\mathrm{M}+\mathrm{H}]^{+}$calcd for $\mathrm{C}_{18} \mathrm{H}_{15} \mathrm{O}_{4} \mathrm{~S}^{+}$: 327.0686, found: 327.0693 .

Ethyl 4-oxo-6-phenyl-2-(thiophen-2-yl)-4H-thiopyran-3-carboxylate (4na)

Brown oil, $\mathrm{R}_{f}=0.34(\mathrm{PE} / \mathrm{EA} 3: 1), 30 \mathrm{mg}$, yield $45 \% .{ }^{1} \mathbf{H}$ NMR $(400 \mathrm{MHz}$, $\left.\mathrm{CDCl}_{3}\right) \delta 7.64-7.58(\mathrm{~m}, 2 \mathrm{H}), 7.56(\mathrm{~d}, J=5.1 \mathrm{~Hz}, 1 \mathrm{H}), 7.53-7.48(\mathrm{~m}$, $3 \mathrm{H}), 7.45(\mathrm{~d}, J=3.3 \mathrm{~Hz}, 1 \mathrm{H}), 7.26(\mathrm{~s}, 1 \mathrm{H}), 7.14(\mathrm{dd}, J=4.8,4.0 \mathrm{~Hz}, 1 \mathrm{H})$, $4.31(\mathrm{q}, J=7.1 \mathrm{~Hz}, 2 \mathrm{H}), 1.23(\mathrm{t}, J=7.1 \mathrm{~Hz}, 3 \mathrm{H}) .{ }^{13} \mathbf{C}$ NMR $(101 \mathrm{MHz}$, $\left.\mathrm{CDCl}_{3}\right) \delta 178.8,171.7,165.7,152.9,144.1,135.3,135.0,131.1,130.1$, $129.7,129.5,128.2,127.1,126.8,62.1,13.9$. HRMS (ESI) $\mathrm{m} / \mathrm{z}[\mathrm{M}+\mathrm{H}]^{+}$ calcd for $\mathrm{C}_{18} \mathrm{H}_{15} \mathrm{O}_{3} \mathrm{~S}_{2}^{+}: 343.0457$, found: 343.0461 .

Methyl 2-methyl-4-oxo-6-phenyl-4H-thiopyran-3-carboxylate (4oa) Brown oil, $\mathrm{R}_{f}=0.31$ (PE/EA 3:1), $30 \mathrm{mg}$, yield 58\%. ${ }^{1} \mathbf{H}$ NMR $(400 \mathrm{MHz}$, $\left.\mathrm{CDCl}_{3}\right) \delta 7.50(\mathrm{dd}, J=7.7,1.8 \mathrm{~Hz}, 2 \mathrm{H}), 7.47-7.41(\mathrm{~m}, 3 \mathrm{H}), 7.13(\mathrm{~s}, 1 \mathrm{H})$, 3.88 (s, 3H), 2.38 (s, 3H). ${ }^{13} \mathbf{C}$ NMR $\left(101 \mathrm{MHz}, \mathrm{CDCl}_{3}\right) \delta 178.6,166.3$, 152.3, 149.8, 135.3, 131.0, 129.4, 127.1, 126.9, 126.7, 52.8, 20.8. HRMS (ESI) $\mathrm{m} / \mathrm{z}[\mathrm{M}+\mathrm{Na}]^{+}$calcd for $\mathrm{C}_{14} \mathrm{H}_{12} \mathrm{NaO}_{3} \mathrm{~S}^{+}$: 283.0399, found: 283.0406 .

\section{2,3,6-Triphenyl-4H-thiopyran-4-one (4pa)}

Brown oil, $\mathrm{R}_{f}=0.5$ (PE/EA 3:1), $36 \mathrm{mg}$, yield 53\%. ${ }^{1} \mathbf{H}$ NMR $(400 \mathrm{MHz}$, $\left.\mathrm{CDCl}_{3}\right) \delta 7.68(\mathrm{dd}, J=6.5,2.9 \mathrm{~Hz}, 2 \mathrm{H}), 7.57-7.48(\mathrm{~m}, 3 \mathrm{H}), 7.41(\mathrm{~s}, 1 \mathrm{H})$, $7.25-7.16$ (m, 8H), $7.14-7.07$ (m, 2H). ${ }^{13} \mathbf{C} \mathbf{~ N M R}\left(101 \mathrm{MHz}, \mathrm{CDCl}_{3}\right) \delta$ 180.8, 168.1, 151.8, 139.7, 136.4, 135.9, 134.6, 130.8, 130.7, 129.4, 129.3, 129.1, 128.4, 127.8, 127.6, 127.4, 126.7. HRMS (ESI) m/z [M+H] ${ }^{+}$calcd for $\mathrm{C}_{23} \mathrm{H}_{17} \mathrm{OS}^{+}: 341.0995$, found: 341.1000 .

Ethyl 2-cyclobutyl-4-oxo-6-phenyl-4H-thiopyran-3-carboxylate (4qu) Brown oil, $\mathrm{R}_{f}=0.3$ (PE/EA 3:1), $32 \mathrm{mg}$, yield 50\%. ${ }^{1} \mathbf{H}$ NMR $(400 \mathrm{MHz}$, $\left.\mathrm{CDCl}_{3}\right) \delta 6.82(\mathrm{~s}, 1 \mathrm{H}), 4.35(\mathrm{q}, J=7.1 \mathrm{~Hz}, 2 \mathrm{H}), 3.66(\mathrm{p}, J=9.0 \mathrm{~Hz}, 1 \mathrm{H})$, $2.54-2.38(\mathrm{~m}, 1 \mathrm{H}), 2.32-2.23(\mathrm{~m}, 4 \mathrm{H}), 2.06-1.80(\mathrm{~m}, 6 \mathrm{H}), 1.73-1.70$ (m, 1H), $1.57-1.33(\mathrm{~m}, 7 \mathrm{H}), 1.32-1.12(\mathrm{~m}, 1 \mathrm{H}) .{ }^{13} \mathbf{C}$ NMR $(101 \mathrm{MHz}$, $\left.\mathrm{CDCl}_{3}\right) \delta 178.7,166.2,160.3,157.3,132.5,126.1,61.7,46.0,39.2,33.3$, 28.9, 26.2, 25.5, 18.2, 14.1. HRMS (ESI) $\mathrm{m} / \mathrm{z}[\mathrm{M}+\mathrm{H}]^{+}$calcd for $\mathrm{C}_{18} \mathrm{H}_{25} \mathrm{O}_{3} \mathrm{~S}^{+}: 321.1519$, found: 321.1525 . 
<smiles>CCOC(=O)c1c(C2CC2)sc(C2CCCCC2)cc1=O</smiles>

Ethyl 6-cyclohexyl-2-cyclopropyl-4-oxo-4H-thiopyran-3-carboxylate (4ru)

Brown oil, $\mathrm{R}_{f}=0.23\left(\right.$ PE/EA 3:1), $27 \mathrm{mg}$, yield 44\%. ${ }^{1} \mathbf{H}$ NMR $(400 \mathrm{MHz}$, $\left.\mathrm{CDCl}_{3}\right) \delta 6.80(\mathrm{~s}, 1 \mathrm{H}), 4.40(\mathrm{q}, J=7.1 \mathrm{~Hz}, 2 \mathrm{H}), 2.40(\mathrm{tt}, J=11.7,3.1 \mathrm{~Hz}$, $1 \mathrm{H}), 2.09-1.97(\mathrm{~m}, 1 \mathrm{H}), 1.93-1.80(\mathrm{~m}, 4 \mathrm{H}), 1.76-1.70(\mathrm{~m}, 1 \mathrm{H}), 1.51-$ $1.28(\mathrm{~m}, 6 \mathrm{H}), 1.31-1.28(\mathrm{~m}, 2 \mathrm{H}), 1.09-0.99(\mathrm{~m}, 2 \mathrm{H}), 1.00-0.84(\mathrm{~m}$, 2H). ${ }^{13} \mathbf{C}$ NMR $\left(101 \mathrm{MHz}, \mathrm{CDCl}_{3}\right) \delta 178.4,166.3,159.8,155.3,135.2$, $126.1,61.7,45.9,33.2,26.2,25.5,15.5,14.2,8.8$. HRMS (ESI) m/z $[\mathrm{M}+\mathrm{H}]^{+}$calcd for $\mathrm{C}_{17} \mathrm{H}_{23} \mathrm{O}_{3} \mathrm{~S}^{+}: 307.1362$, found: 307.1368 .<smiles>CCOC(=O)c1c(-c2ccccc2)sc(-c2ccc3c(c2)CC[C@@H]2[C@@H]3CC[C@]3(C)C(=O)CC[C@H]23)cc1=O</smiles>

Ethyl 6-((8R,9S,13S,14S)-13-methyl-17-oxo-7,8,9,11,12,13,14,15,16,17-decahydro-6Hcyclopenta[ $[a]$ phenanthren-3-yl)-4-oxo-2-phenyl-4H-thiopyran-3-carboxylate (13) Yellow oil, $\mathrm{R}_{f}=0.29(\mathrm{PE} / \mathrm{EA} 3: 1), 64 \mathrm{mg}$, yield 62\%. ${ }^{1} \mathbf{H}$ NMR $\left(400 \mathrm{MHz}, \mathrm{CDCl}_{3}\right) \delta 7.53(\mathrm{~d}, J=7.3$ $\mathrm{Hz}, 2 \mathrm{H}), 7.47$ (t, J = 7.3 Hz, 3H), 7.40 (s, 2H), 7.35 (s, 1H), 7.29 (s, 1H), 4.14 (q, J = 7.1 Hz, 2H), $3.03-2.89(\mathrm{~m}, 2 \mathrm{H}), 2.53(\mathrm{dd}, J=19,8 \mathrm{~Hz}, 1 \mathrm{H}), 2.44-2.38(\mathrm{~m}, 1 \mathrm{H}), 2.31(\mathrm{td}, J=11,4 \mathrm{~Hz}, 1 \mathrm{H}), 2.17$ $(\mathrm{dt}, J=19,9 \mathrm{~Hz}, 1 \mathrm{H}), 2.11-2.03(\mathrm{~m}, 2 \mathrm{H}), 2.02-1.96(\mathrm{~m}, 1 \mathrm{H}), 1.68-1.48(\mathrm{~m}, 6 \mathrm{H}), 1.02(\mathrm{t}, J=7.1$ $\mathrm{Hz}, 3 \mathrm{H}), 0.92$ (s, 3H). ${ }^{13} \mathbf{C}$ NMR (101 MHz, $\left.\mathrm{CDCl}_{3}\right) \delta 220.4,178.6,165.4,152.9,152.0,143.3,137.9$, 134.6, 134.2, 132.9, 130.5, 128.9, 128.1, 127.2, 126.7, 126.5, 124.0, 61.7, 50.4, 47.9, 44.4, 37.8, 35.8, 31.5, 29.3, 26.2, 25.6, 21.5, 13.8, 13.7. HRMS (ESI) m/z $[\mathrm{M}+\mathrm{H}]^{+}$calcd for $\mathrm{C}_{32} \mathrm{H}_{33} \mathrm{O}_{4} \mathrm{~S}^{+}: 513.2094$, found: 513.2099 .

\subsection{An example for a 1-mmol scale reaction}

A mixture of thiadiazole (2a, $234 \mathrm{mg}, 1.0 \mathrm{mmol}),[\mathrm{Rh}(\mathrm{COD}) \mathrm{Cl}]_{2}(50 \mathrm{mg}, 0.10 \mathrm{mmol}), \mathrm{DPPF}(140 \mathrm{mg}$, $0.24 \mathrm{mmol}$ ), and $\mathrm{AgSbF}_{6}(90 \mathrm{mg}, 0.24 \mathrm{mmol}$ ) was added to a flame-dried 10-mL Schlenk tube charged with a magnetic stirring bar. The Schlenk tube was sealed with rubber stopper immediately and protected with nitrogen by evacuation-backfill operations for three times. Then $3 \mathrm{~mL}$ of fresh distilled $\mathrm{PhCl}$ was injected to the system via a syringe. After that, ynal $1 \mathbf{a}(260 \mathrm{mg}, 2.0 \mathrm{mmol})$ was dissolved in fresh distilled $\mathrm{PhCl}(3 \mathrm{~mL})$ and the solutions were added to the tube via a syringe. The mixture was stirred at $130{ }^{\circ} \mathrm{C}$ for $24 \mathrm{~h}$. The solvent was evaporated under reduced pressure, and the crude mixture was subjected to flash chromatography on silica gel, affording the corresponding product $\mathbf{4 a a}(131 \mathrm{mg}, 40 \%)$ as brown oil.

\section{Experimental procedure for the reactions of thiodiazoles 2 and enals 4}

\section{General procedure:}




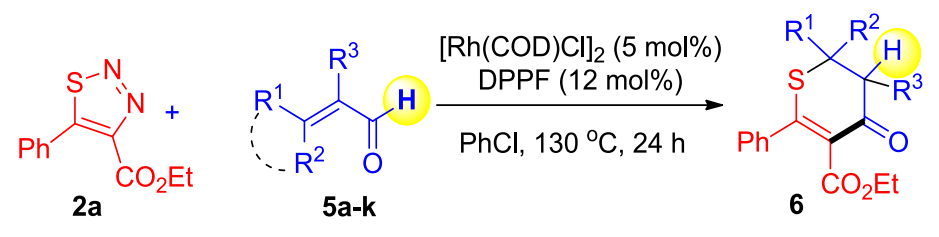

Scheme S9. Reactions of thiodiazoles $\mathbf{2 a}$ and ynals $\mathbf{5}$

Procedure of synthesis of $\mathbf{6}$ is similar with that of $\mathbf{4}$ with variation of amount of $[\mathrm{Rh}(\mathrm{COD}) \mathrm{Cl}]_{2}$ and DPPF.

To a dry reaction tube charged with a magnetic stirring bar, thiadiazole (2a, $46 \mathrm{mg}, 0.200 \mathrm{mmol})$, $[\mathrm{Rh}(\mathrm{COD}) \mathrm{Cl}]_{2}(5 \mathrm{mg}, 0.010 \mathrm{mmol})$ and DPPF (14 mg, $\left.0.024 \mathrm{mmo}\right)$ under nitrogen atmosphere was slowly added a solution of enal substrate 5a-k $(0.400 \mathrm{mmol}, 2.0$ equiv $)$ in freshly distilled $\mathrm{PhCl}(1.5$ $\mathrm{mL}$ ) via a syringe. The mixtrure was heated at $130{ }^{\circ} \mathrm{C}$ for 24 hours. Upon completion of the reaction, the solvent was evaporated under reduced pressure, and the crude mixture was subjected to flash chromatography on silica gel affording the corresponding product 6.<smiles>CCOC(=O)C1=C(c2ccccc2)SC(c2ccccc2)CC1=O</smiles>

$6 a a$<smiles>CCOC(=O)C1=C(c2ccccc2)SC(C[C@H](C)CCC=C(C)C)CC1=O</smiles><smiles>CCOC(=O)C1=C(c2ccccc2)SC(C)(C)CC1=O</smiles>

Ethyl 4-oxo-2,6-diphenyl-3,4-dihydro-2H-thiopyran-5-carboxylate (6aa) Brown oil, $\mathrm{R}_{f}=0.40$ (PE/EA 5:1), $41 \mathrm{mg}$, yield 61\%. ${ }^{1} \mathbf{H}$ NMR (400 MHz, $\left.\mathrm{CDCl}_{3}\right) \delta 7.65-7.34(\mathrm{~m}, 10 \mathrm{H}), 4.81(\mathrm{dd}, J=14.4,2.4 \mathrm{~Hz}, 1 \mathrm{H}), 4.02(\mathrm{qd}, J=7.1$, $3.1 \mathrm{~Hz}, 2 \mathrm{H}), 3.25(\mathrm{~d}, J=15.3 \mathrm{~Hz}, 1 \mathrm{H}), 3.04(\mathrm{dd}, J=15.9,2.3 \mathrm{~Hz}, 1 \mathrm{H}), 0.94(\mathrm{t}, J$ $=7.1 \mathrm{~Hz}, 3 \mathrm{H}) .{ }^{13} \mathbf{C}$ NMR $\left(101 \mathrm{MHz}, \mathrm{CDCl}_{3}\right) \delta 191.0,165.8,162.0,136.9,136.1$, 130.7, 129.1, 128.8, 128.5, 127.9, 127.4, 61.2, 46.2, 43.6, 13.6. HRMS (ESI) m/z $[\mathrm{M}+\mathrm{H}]^{+}$calcd for $\mathrm{C}_{20} \mathrm{H}_{19} \mathrm{O}_{3} \mathrm{~S}^{+}: 339.1049$, found: 339.1052 .

\section{Ethyl 2-((S)-2,6-dimethylhept-5-en-1-yl)-4-oxo-6-phenyl-3,4-dihydro-2H-} thiopyran-5-carboxylate (6ab)

Brown oil, $\mathrm{R}_{f}=0.20$ (PE/EA 10:1), $48 \mathrm{mg}$, yield 62\%. ${ }^{1} \mathbf{H}$ NMR (400 MHz, $\left.\mathrm{CDCl}_{3}\right) \delta 7.54-7.32(\mathrm{~m}, 5 \mathrm{H}), 5.12-5.04(\mathrm{~m}, 1 \mathrm{H}), 3.98(\mathrm{qd}, J=7.1,2.8 \mathrm{~Hz}, 2 \mathrm{H})$, $3.78-3.57(\mathrm{~m}, 1 \mathrm{H}), 2.86(\mathrm{ddd}, J=15.8,9.0,3.1 \mathrm{~Hz}, 1 \mathrm{H}), 2.75-2.55(\mathrm{~m}, 1 \mathrm{H})$, $2.13-1.90(\mathrm{~m}, 2 \mathrm{H}), 1.88-1.77(\mathrm{~m}, 5 \mathrm{H}), 1.60(\mathrm{~d}, J=5.4 \mathrm{~Hz}, 3 \mathrm{H}), 1.52-1.12$ $(\mathrm{m}, 3 \mathrm{H}), 0.92(\mathrm{dt}, J=14.3,6.8 \mathrm{~Hz}, 6 \mathrm{H}) .{ }^{13} \mathbf{C} \mathbf{N M R}\left(101 \mathrm{MHz}, \mathrm{CDCl}_{3}\right) \delta$ 191.3, 165.9, 161.9 (isomer a), 161.6 (isomer b), 136.61, 136.55, 131.7, 130.6, 128.5, 127.9, 124.1, 61.1, 43.9 (isomer a), 43.2 (isomer b), 40.9, 40.3 (isomer a), 40.0 (isomer b), 37.1 (isomer a), 36.3 (isomer b), 29.4 (isomer a), 29.3 (isomer b), 25.7, 25.2 (isomer a), 25.1 (isomer b), 19.5 (isomer a), 19.1 (isomer b), 17.7, 13.6. HRMS (ESI) m/z [M+H] $]^{+}$calcd for $\mathrm{C}_{23} \mathrm{H}_{31} \mathrm{O}_{3} \mathrm{~S}^{+}: 387.1988$, found: 387.1988.

Ethyl 2,2-dimethyl-4-oxo-6-phenyl-3,4-dihydro-2H-thiopyran-5carboxylate (6ac)

Brown solid, $\mathrm{R}_{f}=0.29$ (PE/EA 5:1), M.p. 95-96 ${ }^{\circ} \mathrm{C}, 40 \mathrm{mg}$, yield 70\%. ${ }^{1} \mathbf{H}$ NMR $\left(400 \mathrm{MHz}, \mathrm{CDCl}_{3}\right) \delta 7.57-7.32(\mathrm{~m}, 5 \mathrm{H}), 3.98(\mathrm{q}, J=7.1 \mathrm{~Hz}, 2 \mathrm{H}), 2.76(\mathrm{~s}, 2 \mathrm{H})$, $1.53(\mathrm{~s}, 6 \mathrm{H}), 0.90(\mathrm{t}, J=7.1 \mathrm{~Hz}, 3 \mathrm{H}) .{ }^{13} \mathbf{C} \mathbf{N M R}\left(101 \mathrm{MHz}, \mathrm{CDCl}_{3}\right) \delta 191.5$, 165.9, 160.8, 136.5, 130.5, 128.5, 127.9, 126.9, 61.1, 51.3, 45.6, 27.8, 13.6. HRMS (ESI) m/z [M+H] $]^{+}$calcd for $\mathrm{C}_{16} \mathrm{H}_{19} \mathrm{O}_{3} \mathrm{~S}^{+}: 291.1049$, found: 291.1045. 
<smiles>CCOC(=O)C1=C(c2ccccc2)SC2(CCC2)CC1=O</smiles><smiles>CCOC(=O)C1=C(c2ccccc2)SC2(C=C1C(=O)OC)CCCC2</smiles><smiles>CCOC(=O)C1=C(c2ccccc2)SC2(C=C1)CCCCC2</smiles>

6af<smiles>CCOC(=O)C1=C(c2ccccc2)SC2(CCN(Cc3ccccc3)CC2)CC1=O</smiles><smiles>CCOC(=O)C1=C(c2ccccc2)SC2(CCCCCC2)CC1=O</smiles>
6ah
Ethyl 8-oxo-6-phenyl-5-thiaspiro[3.5]non-6-ene-7-carboxylate (6ad)

Yellow oil, $\mathrm{R}_{f}=0.29(\mathrm{PE} / \mathrm{EA} 5: 1), 45 \mathrm{mg}$, yield 74\%. ${ }^{\mathbf{1}} \mathbf{H} \mathbf{N M R}(400 \mathrm{MHz}$, $\left.\mathrm{CDCl}_{3}\right) \delta 7.68-7.31(\mathrm{~m}, 5 \mathrm{H}), 3.96(\mathrm{q}, J=7.1 \mathrm{~Hz}, 2 \mathrm{H}), 2.98(\mathrm{~s}, 2 \mathrm{H}), 2.49-2.21$ $(\mathrm{m}, 4 \mathrm{H}), 2.20-1.97(\mathrm{~m}, 2 \mathrm{H}), 0.87(\mathrm{t}, J=7.1 \mathrm{~Hz}, 3 \mathrm{H}) .{ }^{13} \mathbf{C}$ NMR $(101 \mathrm{MHz}$, $\left.\mathrm{CDCl}_{3}\right) \delta 191.4,165.8,160.8,136.3,130.4,128.4,127.8,127.5,61.0,48.7,48.0$, 34.3, 15.9, 13.5. HRMS (ESI) $\mathrm{m} / \mathrm{z}[\mathrm{M}+\mathrm{H}]^{+}$calcd for $\mathrm{C}_{17} \mathrm{H}_{19} \mathrm{O}_{3} \mathrm{~S}^{+}:$303.1049, found: 303.1049 .

\section{Ethyl 9-oxo-7-phenyl-6-thiaspiro[4.5]dec-7-ene-8-carboxylate (6ae)}

Yellow oil, $\mathrm{R}_{f}=0.29$ (PE/EA 5:1), $52 \mathrm{mg}$, yield 82\%. ${ }^{1} \mathbf{H}$ NMR (400 MHz, $\left.\mathrm{CDCl}_{3}\right) \delta 7.54-7.33(\mathrm{~m}, 5 \mathrm{H}), 3.98(\mathrm{q}, J=7.1 \mathrm{~Hz}, 2 \mathrm{H}), 2.87$ (s, 2H), $2.14-2.04$ $(\mathrm{m}, 2 \mathrm{H}), 1.97-1.73(\mathrm{~m}, 6 \mathrm{H}), 0.90(\mathrm{t}, J=7.1 \mathrm{~Hz}, 3 \mathrm{H}) .{ }^{13} \mathbf{C}$ NMR $(101 \mathrm{MHz}$, $\left.\mathrm{CDCl}_{3}\right) \delta 191.7,166.0,161.9,136.6,130.5,128.5,128.0,127.4,61.1,55.4,49.5$, 38.8, 24.0, 13.6. HRMS (ESI) $\mathrm{m} / \mathrm{z}[\mathrm{M}+\mathrm{H}]^{+}$calcd for $\mathrm{C}_{18} \mathrm{H}_{21} \mathrm{O}_{3} \mathrm{~S}^{+}$: 317.1206, found: 317.1210 .

Ethyl 4-oxo-2-phenyl-1-thiaspiro[5.5] undec-2-ene-3-carboxylate (6af) Yellow oil, $\mathrm{R}_{f}=0.30\left(\mathrm{PE} / \mathrm{EA} \mathrm{5:1),} 43 \mathrm{mg}\right.$, yield 65\%. ${ }^{1} \mathbf{H}$ NMR (400 MHz, $\left.\mathrm{CDCl}_{3}\right) \delta 7.53-7.30(\mathrm{~m}, 5 \mathrm{H}), 3.96(\mathrm{q}, J=7.1 \mathrm{~Hz}, 2 \mathrm{H}), 2.80(\mathrm{~s}, 2 \mathrm{H}), 2.06(\mathrm{~d}, J=$ $13.2 \mathrm{~Hz}, 2 \mathrm{H}), 1.76-1.45(\mathrm{~m}, 8 \mathrm{H}), 0.88(\mathrm{t}, J=7.1 \mathrm{~Hz}, 3 \mathrm{H}) .{ }^{13} \mathbf{C}$ NMR (101 MHz, $\left.\mathrm{CDCl}_{3}\right) \delta 191.6,165.9,160.4,136.8,130.4,128.4,127.9,127.0,61.0,50.5,50.4$, 35.8, 25.4, 21.9, 13.6. HRMS (ESI) $\mathrm{m} / \mathrm{z}[\mathrm{M}+\mathrm{H}]^{+}$calcd for $\mathrm{C}_{19} \mathrm{H}_{23} \mathrm{O}_{3} \mathrm{~S}^{+}$: 331.1362 , found: 331.1370 .

Ethyl 9-benzyl-4-oxo-2-phenyl-1-thia-9-azaspiro[5.5] undec-2-ene-3carboxylate (6ag)

Brown oil, $\mathrm{R}_{f}=0.30(\mathrm{PE} / \mathrm{EA} 5: 1), 61 \mathrm{mg}$, yield 73\%. ${ }^{\mathbf{1}} \mathbf{H} \mathbf{N M R}\left(400 \mathrm{MHz}, \mathrm{CDCl}_{3}\right)$ $\delta 7.51-7.24(\mathrm{~m}, 10 \mathrm{H}), 3.98(\mathrm{q}, J=7.1 \mathrm{~Hz}, 2 \mathrm{H}), 3.56(\mathrm{~s}, 2 \mathrm{H}), 2.83(\mathrm{~s}, 2 \mathrm{H}), 2.73$ $(\mathrm{d}, J=12.1 \mathrm{~Hz}, 2 \mathrm{H}), 2.48(\mathrm{t}, J=10.6 \mathrm{~Hz}, 2 \mathrm{H}), 2.10(\mathrm{~d}, J=13.8 \mathrm{~Hz}, 2 \mathrm{H}), 1.93-$ $1.81(\mathrm{~m}, 2 \mathrm{H}), 0.89(\mathrm{t}, J=7.1 \mathrm{~Hz}, 3 \mathrm{H}) .{ }^{13} \mathbf{C} \mathbf{N M R}\left(101 \mathrm{MHz}, \mathrm{CDCl}_{3}\right) \delta$ 191.3, 165.8, 159.6, 138.0, 136.6, 130.6, 129.0, 128.5, 128.3, 128.0, 127.4, 127.2, 63.0, 61.1, 49.1, 48.5, 35.4, 29.7, 13.6. HRMS (ESI) $\mathrm{m} / \mathrm{z}[\mathrm{M}+\mathrm{H}]^{+}$calcd for $\mathrm{C}_{25} \mathrm{H}_{28} \mathrm{NO}_{3} \mathrm{~S}^{+}:$422.1784, found: 422.1794 .

Ethyl 4-oxo-2-phenyl-1-thiaspiro[5.6]dodec-2-ene-3-carboxylate (6ah) Brown oil, $\mathrm{R}_{f}=0.49$ (PE/EA 5:1), $29 \mathrm{mg}$, yield 42\%. ${ }^{1} \mathbf{H}$ NMR (400 MHz, $\left.\mathrm{CDCl}_{3}\right) \delta 7.52-7.15(\mathrm{~m}, 5 \mathrm{H}), 3.98(\mathrm{q}, J=7.1 \mathrm{~Hz}, 2 \mathrm{H}), 2.81(\mathrm{~s}, 2 \mathrm{H}), 2.10$ (dd, $J$ $=14.7,7.8 \mathrm{~Hz}, 2 \mathrm{H}), 1.81(\mathrm{dd}, J=14.7,8.2 \mathrm{~Hz}, 2 \mathrm{H}), 1.73-1.55(\mathrm{~m}, 8 \mathrm{H}), 0.89(\mathrm{t}$, $J=7.1 \mathrm{~Hz}, 3 \mathrm{H}) .{ }^{13} \mathbf{C} \mathbf{N M R}\left(101 \mathrm{MHz}, \mathrm{CDCl}_{3}\right) \delta 191.7,166.0,160.7,136.6,130.4$, 128.4, 127.9, 127.1, 61.1, 53.0, 51.3, 38.7, 29.5, 22.8, 13.6. HRMS (ESI) m/z $[\mathrm{M}+\mathrm{H}]^{+}$calcd for $\mathrm{C}_{20} \mathrm{H}_{25} \mathrm{O}_{3} \mathrm{~S}^{+}: 345.1519$, found: 345.1514 . 
<smiles>CCCCCCCCCCCCC1(CCCCCC)CC(=O)C(C(=O)OCC)=C(c2ccccc2)S1</smiles>

6ai

Ethyl 4-oxo-2-phenyl-1-thiaspiro[5.11]heptadec-2-ene-3-carboxylate (6ai) Brown oil, $\mathrm{R}_{f}=0.50$ (PE/EA 5:1), $36 \mathrm{mg}$, yield 44\%. ${ }^{1} \mathbf{H}$ NMR (400 MHz, $\left.\mathrm{CDCl}_{3}\right) \delta 7.56-7.30(\mathrm{~m}, 5 \mathrm{H}), 3.98(\mathrm{q}, J=7.1 \mathrm{~Hz}, 2 \mathrm{H}), 2.80(\mathrm{~s}, 2 \mathrm{H}), 1.84(\mathrm{~d}, J=$ $8.9 \mathrm{~Hz}, 4 \mathrm{H}), 1.52-1.18(\mathrm{~m}, 18 \mathrm{H}), 0.89(\mathrm{t}, J=7.1 \mathrm{~Hz}, 3 \mathrm{H}) .{ }^{13} \mathbf{C}$ NMR $(101 \mathrm{MHz}$, $\left.\mathrm{CDCl}_{3}\right) \delta 191.6,166.0,160.6,136.8,130.4,128.4,127.9,127.1,61.0,52.7,48.4$, 32.0, 26.0, 25.9, 22.4, 21.8, 18.9, 13.6. HRMS (ESI) $\mathrm{m} / \mathrm{z}[\mathrm{M}+\mathrm{H}]^{+}$calcd for $\mathrm{C}_{25} \mathrm{H}_{35} \mathrm{O}_{3} \mathrm{~S}^{+}:$415.2301, found: 415.2303 .

Ethyl 8-methyl-4-oxo-2-phenyl-1-thiaspiro[5.14]icos-2-ene-3-carboxylate (6aj)<smiles>CCOC(=O)C1=C(c2ccccc2)S[C@@]2(CCCCCCCCCCCCC(C)C2)CC1=O</smiles>

6aj<smiles>CCOC(=O)C1=C(c2ccccc2)SC2CCCC2C1=O</smiles>

6ak

Colorless oil, $\mathrm{R}_{f}=0.55$ (PE/EA 5:1), $45 \mathrm{mg}$, yield 48\%. ${ }^{\mathbf{1}} \mathbf{H}$ NMR $(400 \mathrm{MHz}$, $\left.\mathrm{CDCl}_{3}\right) \delta 7.53-7.31(\mathrm{~m}, 5 \mathrm{H}), 3.98(\mathrm{qd}, J=7.1,4.6 \mathrm{~Hz}, 2 \mathrm{H}), 2.96-2.65(\mathrm{~m}, 2 \mathrm{H})$, $1.96-1.75(\mathrm{~m}, 3 \mathrm{H}), 1.68(\mathrm{~s}, 2 \mathrm{H}), 1.48-1.17(\mathrm{~m}, 21 \mathrm{H}), 1.06(\mathrm{~d}, J=6.1 \mathrm{~Hz}, 2 \mathrm{H})$, $1.00-0.81(\mathrm{~m}, 5 \mathrm{H}) .{ }^{13} \mathbf{C}$ NMR (101 MHz, $\left.\mathrm{CDCl}_{3}\right) \delta 191.8$ (isomer a), 191.7 (isomer b), 165.93 (isomer a), 165.89 (isomer b), 160.82 (isomer a), 160.76 (isomer b), 136.82 (isomer b), 136.76 (isomer a), 130.4 (isomer a+b), 128.5 (isomer a+b), 127.93 (isomer a), 127.88 (isomer b), 127.0 (isomer b), 126.8 (isomer a), 61.1 (isomer a+b), 53.6 (isomer a), 53.1 (isomer b), 49.1 (isomer b), 48.8 (isomer a), 44.1 (isomer a), 43.7 (isomer b), 37.2 (isomer b), 37.0 (isomer a), 36.3 (isomer a), 36.0 (isomer b), 34,8 (isomer a), 34.4 (isomer b), 28.5 (isomer b), 28.2 (isomer a), 27.2, 27.0, 26.85, 26.8, 26.62, 26.58, 26.1, 26.0 (isomer a), 25.9 (isomer b), 25.8, 25.7, 25.6, 25.41 (isomer b), 25.37 (isomer a), 24.9 (isomer b), 24.7 (isomer a), 22.5 (isomer a), 22.2 (isomer b), 22.0 (isomer b), 21.9 (isomer a), 13.6 (isomer a+b). HRMS (ESI) m/z $[\mathrm{M}+\mathrm{H}]^{+}$calcd for $\mathrm{C}_{29} \mathrm{H}_{43} \mathrm{O}_{3} \mathrm{~S}^{+}$: 471.2927, found: 471.2937 .

Ethyl 4-oxo-2-phenyl-4,4a,5,6,7,7a-hexahydrocyclopenta[b]thiopyran-3carboxylate (6ak)

Brown oil, $\mathrm{R}_{f}=0.40(\mathrm{PE} / \mathrm{EA} 5: 1), 23 \mathrm{mg}$, yield 38\%. ${ }^{1} \mathbf{H} \mathbf{N M R}\left(400 \mathrm{MHz}, \mathrm{CDCl}_{3}\right)$ $\delta 7.74-7.32(\mathrm{~m}, 5 \mathrm{H}), 4.23-3.80(\mathrm{~m}, 3 \mathrm{H}), 2.86(\mathrm{dt}, J=14.2,8.2 \mathrm{~Hz}, 1 \mathrm{H}), 2.41-$ $2.31(\mathrm{~m}, 1 \mathrm{H}), 2.22-2.05(\mathrm{~m}, 2 \mathrm{H}), 2.02-1.90(\mathrm{~m}, 2 \mathrm{H}), 2.00-1.73(\mathrm{~m}, 1 \mathrm{H}), 0.91$ $(\mathrm{t}, J=7.1 \mathrm{~Hz}, 3 \mathrm{H}) .{ }^{13} \mathrm{C} \mathbf{N M R}\left(101 \mathrm{MHz}, \mathrm{CDCl}_{3}\right) \delta 193.1,166.1,159.5,136.6$, $130.4,128.5,127.7,126.0,61.2,49.0,46.1,31.8,27.1,21.9,13.6$. HRMS (ESI) $\mathrm{m} / \mathrm{z}[\mathrm{M}+\mathrm{H}]^{+}$calcd for $\mathrm{C}_{17} \mathrm{H}_{19} \mathrm{O}_{3} \mathrm{~S}^{+}:$303.1049, found: 303.1052 .

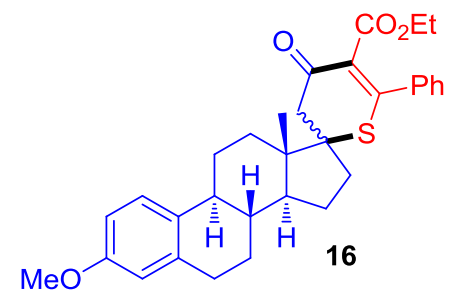

$(8 R, 9 S, 13 S, 14 S)$-Ethyl 3-methoxy-13-methyl-4'-oxo-6'-phenyl-3',4',6,7,8,9,11,12,13,14,15,16dodecahydrospiro[cyclopenta[ $a]$ phenanthrene-17,2'-thiopyran]-5'-carboxylate (16) 
White solid, $\mathrm{R}_{f}=0.50$ (PE/EA 5:1), M.p. 83-85 ${ }^{\circ} \mathrm{C}, 77 \mathrm{mg}$, yield 75\%. ${ }^{1} \mathbf{H}$ NMR (400 MHz, $\left.\mathrm{CDCl}_{3}\right)$ $\delta 7.58-7.38(\mathrm{~m}, 5 \mathrm{H}), 7.19(\mathrm{~d}, J=8.6 \mathrm{~Hz}, 1 \mathrm{H}), 6.72(\mathrm{dd}, J=8.5,2.2 \mathrm{~Hz}, 1 \mathrm{H}), 6.64(\mathrm{~s}, 1 \mathrm{H}), 4.00(\mathrm{q}, J$ $=6.6 \mathrm{~Hz}, 2 \mathrm{H}), 3.78(\mathrm{~s}, 3 \mathrm{H}), 3.15-2.67(\mathrm{~m}, 4 \mathrm{H}), 2.40-2.20(\mathrm{~m}, 3 \mathrm{H}), 2.16-1.87(\mathrm{~m}, 6 \mathrm{H}), 1.60-1.35$ (m, 4H), $0.95-0.87(\mathrm{~m}, 6 \mathrm{H}) .{ }^{13} \mathbf{C}$ NMR $\left(101 \mathrm{MHz}, \mathrm{CDCl}_{3}\right) \delta 192.1,165.9,161.6,157.6,137.8,136.7$, 132.0, 130.6, 128.6, 127.9, 126.4, 113.9, 111.6, 63.3, 61.1, 55.2, 50.1, 48.2, 45.1, 43.5, 39.3, 36.5, 33.9, 29.8, 27.8, 26.5, 23.6, 14.6, 13.7. HRMS (ESI) m/z $[\mathrm{M}+\mathrm{H}]^{+}$calcd for $\mathrm{C}_{32} \mathrm{H}_{37} \mathrm{O}_{4} \mathrm{~S}^{+}$: 517.2407, found: 517.2401 .

\section{Oxidation of 4aa and its X-Ray Crystallographic Analysis}

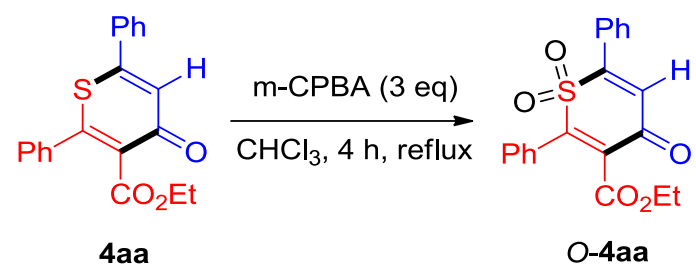

Scheme S10. Oxidation of 4aa with $m$-CPBA

\section{Synthesis of $O-4$ aa}

Ethyl 6-phenyl-2-phenyl-4-oxo-4H-thiopyran-3-carboxylate (4aa, $166 \mathrm{mg}, 0.5 \mathrm{mmol}$ ) was dissolved in $15 \mathrm{~mL}$ of $\mathrm{CHCl}_{3}$ in a round bottom flask, then $m$-CPBA $(85 \%, 305 \mathrm{mg}, 1.5 \mathrm{mmol})$ was added to the solution in four potions in $2 \mathrm{~min}$ at $0{ }^{\circ} \mathrm{C}$. Then the resulting mixture was allowed to reflux for 4 hours. After removal of the solvent, the crude product was purified by flash column chromatography on silica gel $\left(\mathrm{PE} / \mathrm{EA}=6: 1 ; \mathrm{R}_{f}=0.50\right)$ to give $O-4 \mathrm{aa}$ in $50 \%$ yield.

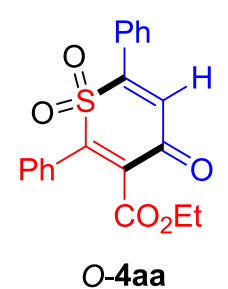

Ehyl 4-oxo-2,6-diphenyl-4H-thiopyran-3-carboxylate 1,1-dioxide ( $O$-4aa)

Yellow solid, $\mathrm{R}_{f}=0.70$ (PE/EA 3:1), M.p. $112-114{ }^{\circ} \mathrm{C}, 90 \mathrm{mg}$, yield 50\%. ${ }^{1} \mathbf{H}$ NMR $\left(400 \mathrm{MHz}, \mathrm{CDCl}_{3}\right) \delta 7.82(\mathrm{~d}, J=7.4 \mathrm{~Hz}, 2 \mathrm{H}), 7.67-7.61(\mathrm{~m}, 2 \mathrm{H}), 7.60-7.38(\mathrm{~m}$, $6 \mathrm{H}), 6.74(\mathrm{~s}, 1 \mathrm{H}), 4.11(\mathrm{q}, J=7.1 \mathrm{~Hz}, 2 \mathrm{H}), 1.00(\mathrm{t}, J=7.1 \mathrm{~Hz}, 3 \mathrm{H}) .{ }^{13} \mathbf{C}$ NMR $(101$ $\left.\mathrm{MHz}, \mathrm{CDCl}_{3}\right) \delta 176.5,161.5,153.4,150.9,133.5,132.1,131.5,129.9,129.3,128.8$, 128.4, 126.7, 125.7, 62.4, 13.6. HRMS (ESI) $\mathrm{m} / \mathrm{z}[\mathrm{M}+\mathrm{H}]^{+}$calcd for $\mathrm{C}_{20} \mathrm{H}_{17} \mathrm{O}_{5} \mathrm{~S}^{+}$: 369.0791, found: 369.0795 .

Yellow crystals of $O-\mathbf{4 a a}$ were grown by slow evaporation of a EA/ PE solution at ambient temperature.

Crystal Data: $\mathrm{C}_{20} \mathrm{H}_{16} \mathrm{O}_{5} \mathrm{~S}, M=368.39$, monoclinic, $a=18.302(2) \AA, b=13.5296(6) \AA, c=$ 15.7947(12) $\AA, \beta=114.012(12)^{\circ}, U=3572.7(5) \AA^{3}, T=108.80$ (10), space group P2/c (no. 13), $Z=$ $8, \mu(\mathrm{Mo} \mathrm{K} \alpha)=0.209,20102$ reflections measured, 7014 unique $\left(R_{\text {int }}=0.0377\right)$ which were used in all calculations. The final $w R\left(F_{2}\right)$ was 0.1001 (all data). 


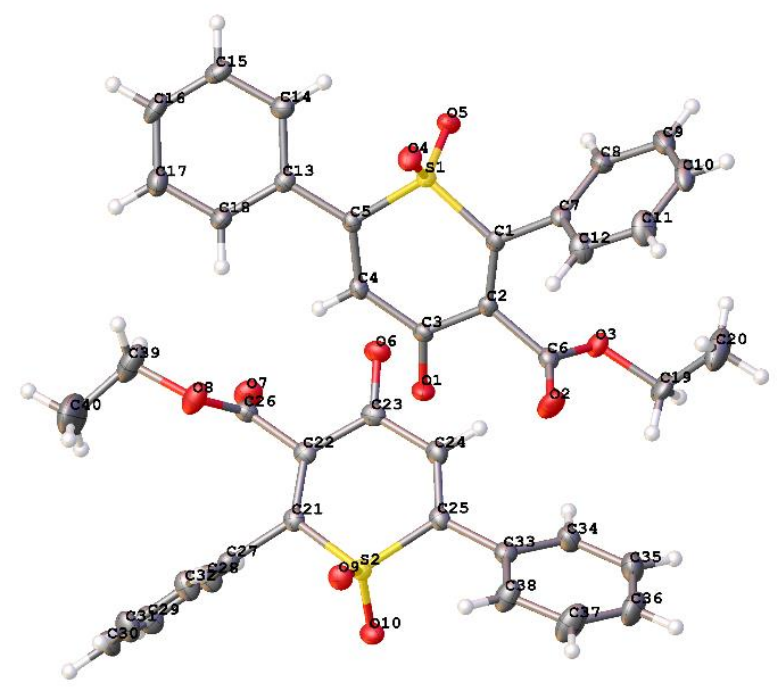

Figure S2. Picture above represents the crystal structure of compound $O-4 a a$. CCDC 1895642 contains the supplementary crystallographic data for this compound. These data can be obtained free of charge from The Cambridge Crystallographic Data Centre via www.ccdc.cam.ac.uk/data_request/cif

Table S2: Crystal data and structure refinement for CCDC 1895642

Identification CODE

Empirical formula

Temperature / $\mathrm{K}$

Crystal system

Space group

$\mathrm{a} / \AA, \mathrm{b} / \AA, \mathrm{c} / \AA$

$\alpha /^{\circ}, \beta /{ }^{\circ}, \gamma /^{\circ}$

Volume / $\AA^{3}$

$\mathrm{Z}$

$\rho_{\text {calc }} / \mathrm{mg} \mathrm{mm}^{-3}$

$\mu / \mathrm{mm}^{-1}$

$\mathrm{F}(000)$

Crystal size $/ \mathrm{mm}^{3}$

$2 \Theta$ range for data collection

Index ranges

Reflections collected

Independent reflections

Data/restraints/parameters

Goodness-of-fit on $\mathrm{F}^{2}$

Final $R$ indexes $\left[I>2 \sigma(I)\right.$ i.e. $\left.F_{o}>4 \sigma\left(F_{o}\right)\right]$

Final $\mathrm{R}$ indexes [all data]

Largest diff. peak/hole / e $\AA^{-3}$

Flack Parameters
CCDC 1895642

$\mathrm{C}_{20} \mathrm{H}_{16} \mathrm{O}_{5} \mathrm{~S}$

108.80(10)

monoclinic

$\mathrm{P} 2 / \mathrm{c}$

18.302(2), 13.5296(6), 15.7947(12)

90.00, 114.012(12), 90.00

$3572.7(5)$

8

1.370

0.209

1536

$0.50 \times 0.40 \times 0.39$

6.5 to $52^{\circ}$

$-16 \leq \mathrm{h} \leq 22,-10 \leq \mathrm{k} \leq 16,-19 \leq 1 \leq 18$

20102

$7014[\mathrm{R}(\mathrm{int})=0.0377($ inf- $0.9 \AA)$ ]

$7014 / 0 / 471$

1.028

$\mathrm{R}_{1}=0.0413, \mathrm{wR}_{2}=0.0927$

$\mathrm{R}_{1}=0.0549, \mathrm{wR}_{2}=0.1001$

$0.295 /-0.519$

$\mathrm{N}$ 


\section{Mechanistic studies}

\subsection{Control experiments}

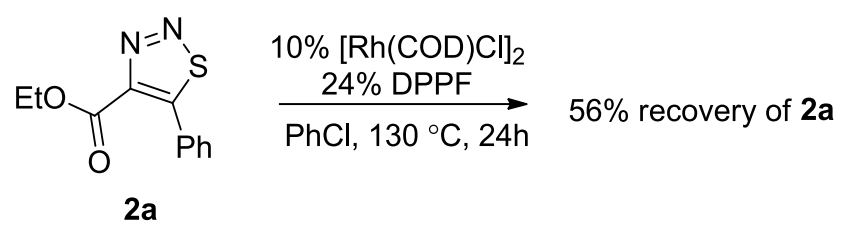

Scheme S11. Reaction of $\mathbf{2 a}$ under catalytic conditoins

A dry reaction tube equipped with a magnetic stirring bar was charged with thiadiazole (2a, 0.200 mmol), $[\mathrm{Rh}(\mathrm{COD}) \mathrm{Cl}]_{2}(10 \mathrm{mg}, 0.020 \mathrm{mmol})$, and DPPF $(27 \mathrm{mg}, 0.048 \mathrm{mmol})$. The tube was sealed immediately and protected with nitrogen by evacuation-backfill operations for three times, then the reaction mixture was allowed to stir for 24 hours. Thiodiazole $2 \mathrm{a}$ was recovered in $56 \%$ yield. The reaction mixture was analyzed by GC-MS, LC-MS, and ${ }^{1} \mathrm{H}$ NMR, but no no identified structures were detected.

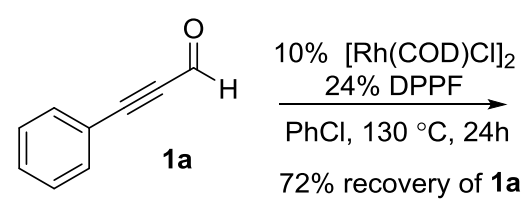

Scheme S12. Reaction of 1a under catalytic conditoins

A dry reaction tube equipped with a magnetic stirring bar was charged with $[\mathrm{Rh}(\mathrm{COD}) \mathrm{Cl}]_{2}(10 \mathrm{mg}$, $0.020 \mathrm{mmol}$ ), and DPPF (27 mg, $0.048 \mathrm{mmol}$ ). The tube was sealed immediately and protected with nitrogen by evacuation-backfill operations for three times, followed by the addition of a solution of ynals 1a $(26 \mathrm{mg}, 0.400 \mathrm{mmol})$ in fresh distilled $\mathrm{PhCl}(1.5 \mathrm{~mL})$ via a syringe. The mixtrure was heated at $130{ }^{\circ} \mathrm{C}$ for 24 hours, then the reaction mixture was allowed to stir for 24 hours. The solvent was evaporated under reduced pressure, and the crude mixture was subjected to flash chromatography on silica gel to recover $\mathbf{1 a}$ in $72 \%$ yield and to afford a trace amount of brownish oil containing S14, S15, and S16, as identified by GC-MS. These byproducts were proposed to be derived from phenylacetylene, the decarbonylation product of 1a. Thus, acyl rhodium hydride intermediate was probably involved.

When 3-( $p$-tolyl)propiolaldehyde (1e) was used as the starting material, similar products were obtained.

7.2 Synthesis of Deuterated 3-phenylpropiolaldehyde $d-1 a^{[10 a]}$ 

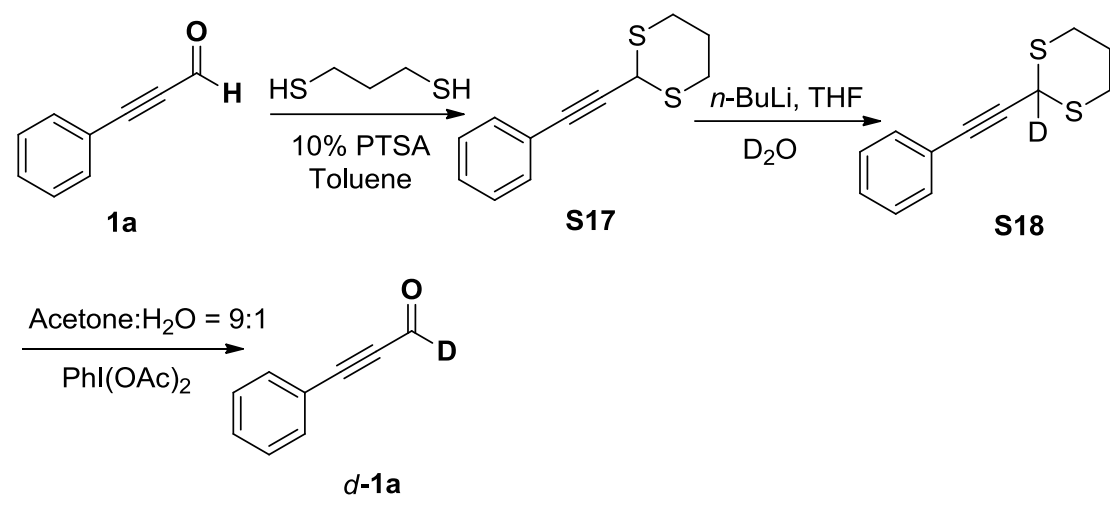

Scheme S13. Synthesis of Deuterated 3-phenylpropiolaldehyde

a) A mixture of 1a (1.30 g, $10 \mathrm{mmol})$, propane-1,3-dithiol (1.30 g, $12 \mathrm{mmol}, 1.2 \mathrm{~mL})$ and PTSA (1 mmol, $190 \mathrm{mg}$ ) was dissolved in toluene $(15 \mathrm{~mL})$, then the mixture was stirred at $60{ }^{\circ} \mathrm{C}$ until the starting material 1a was completely consumed (monitored by TLC). Then the mixture was cooled to room temperature. Finally, the organic solvent was removed in vacuo, and the residue was purified by column chromatography to give the product $\mathbf{S 1 7}$ as yellow oil (1.89 $\mathrm{g}, 86 \%)$.

b) To a stirred solution of $\mathbf{S 1 7}(1.89 \mathrm{~g}, 8.6 \mathrm{mmol}, 1$ equiv) in dry THF $(15 \mathrm{~mL})$ was added $n$-BuLi (1.6 M in hexane solution, $17.2 \mathrm{mmol}, 11 \mathrm{~mL}, 2$ equiv) at $-35^{\circ} \mathrm{C}$ under $\mathrm{N}_{2}$ atmosphere. After stirring for $3 \mathrm{~h}$, excess $\mathrm{D}_{2} \mathrm{O}(0.6 \mathrm{~mL}, 34.4 \mathrm{mmol}, 4$ equiv) was injected into the reaction system. The solution was allowed to stir at $-30^{\circ} \mathrm{C}$ for $8 \mathrm{~h}$. Upon completion of the reaction, the mixture was diluted with water and then extracted with DCM. The combined extracts were concentreated in vacno. The residue was chromatographed on silica gel to give product $\mathbf{S 1 8}$ as yellow oil (190 mg, 10\%).

c) Product $\mathrm{S18}(0.86 \mathrm{mmol}, 190 \mathrm{mg})$ was dissolved in mixed solvent $(20 \mathrm{~mL})$ of acetone and $\mathrm{H}_{2} \mathrm{O}$ $(v: v=9: 1)$, then $\mathrm{PhI}(\mathrm{OAc})_{2}(692 \mathrm{mg}, 2.15 \mathrm{mmol})$ was added to the solution. The mixture was allowed to react for $30 \mathrm{~min}$ at room temperature. When the reaction completed (monitored by TLC), acetone was removed under reduced pressure, and the residue was extracted with DCM $(3 \times 10 \mathrm{~mL})$. The organic layers were concentreated in vacuo, and $d \mathbf{- 1 a}$ was obtained as yellow oil by column chromatography in $70 \%$ (78 $\mathrm{mg}$ ) yield.

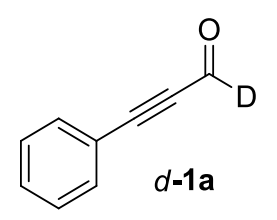

Deuterated 3-phenylpropiolaldehyde $(d-1 a)^{[10 b]}$

Yellow oil, $\mathrm{R}_{f}=0.21\left(\mathrm{CH}_{2} \mathrm{Cl}_{2} / \mathrm{PE} 1: 1\right), 78 \mathrm{mg}$, yield $6 \%$ over 3 steps. ${ }^{1} \mathbf{H}$ NMR $\left(400 \mathrm{MHz}, \mathrm{CDCl}_{3}\right) \delta 7.62-7.60(\mathrm{~m}, 2 \mathrm{H}), 7.52-7.48(\mathrm{~m}, 1 \mathrm{H}), 7.43-7.39(\mathrm{~m}$, $2 \mathrm{H}) .{ }^{13} \mathbf{C ~ N M R}\left(101 \mathrm{MHz}, \mathrm{CDCl}_{3}\right) \delta 133.3,131.3,128.7,119.4,95.1,88.4$ (the carbon signal of carbonyl did not appear). GC-MS (EI) $\mathrm{m} / \mathrm{z}\left(\mathrm{M}^{+}\right)$caled for $\mathrm{C}_{9} \mathrm{H}_{5} \mathrm{DO}: 131.05$, found:131.04.

\subsection{Isotope tracing studies}




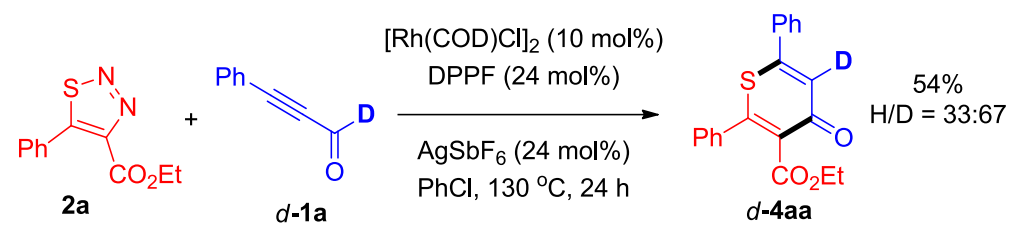

Scheme S14. Reaction of $2 \mathbf{a}$ and $d-1$ a

A flame dried reaction tube equipped with magnetic stirring bars was charged with thiadiazole (2a, $24 \mathrm{mg}, 0.100 \mathrm{mmol}$ ), [Rh(COD)Cl $]_{2}(5 \mathrm{mg}, 0.010 \mathrm{mmol})$, DPPF (14 mg, $\left.0.024 \mathrm{mmol}\right)$, and AgSbF 6 ( $9 \mathrm{mg}, 0.024 \mathrm{mmol})$. Then the reaction tube was sealed immediately and protected with nitrogen by evacuation-backfill operations for three times. Ynal $d-1 \mathbf{a}(26 \mathrm{mg}, 0.200 \mathrm{mmol})$ was dissolved in fresh distilled $\mathrm{PhCl}(1.5 \mathrm{~mL})$ and the solutions were added to the above reaction tube. The mixture were allowed to stir for $24 \mathrm{~h}$. The solvent was evaporated under reduced pressure, and the crude mixture was subjected to flash chromatography on silica gel affording the corresponding product $d$ 4aa as a brown oil.<smiles>CCO[13C](=O)c1c(-c2ccccc2)sc(-c2ccccc2)c([O-])c1=O</smiles>

Ethyl 4-oxo-2,6-diphenyl-4D-thiopyran-3-carboxylate ( $d$-4aa)

Brown oil, $\mathrm{R}_{f}=0.40(\mathrm{PE} / \mathrm{EA} 3: 1), 18 \mathrm{mg}$, yield 54\%. ${ }^{1} \mathbf{H}$ NMR $(400 \mathrm{MHz}$, $\left.\mathrm{CDCl}_{3}\right) \delta 7.61(\mathrm{~d}, J=7.9 \mathrm{~Hz}, 2 \mathrm{H}), 7.59-7.43(\mathrm{~m}, 8 \mathrm{H}), 7.31(\mathrm{~s}, 0.33 \mathrm{H}), 4.14(\mathrm{q}$, $J=7.1 \mathrm{~Hz}, 2 \mathrm{H}), 1.02(\mathrm{t}, J=7.1 \mathrm{~Hz}, 3 \mathrm{H}) .{ }^{13} \mathbf{C} \mathbf{N M R}\left(101 \mathrm{MHz}, \mathrm{CDCl}_{3}\right) \delta 178.6$, $165.3,152.9,152.0,135.4,134.5,134.3$ (very low), 131.0, 130.5, 129.4, 128.9,

$(\mathrm{H} / \mathrm{D}=33: 67) \quad 128.2,127.3,126.8,61.7,13.7$. HRMS (ESI) $\mathrm{m} / \mathrm{z}[\mathrm{M}+\mathrm{H}]^{+}$calcd for $\mathrm{C}_{20} \mathrm{H}_{16} \mathrm{DO}_{3} \mathrm{~S}^{+}: 338.0956$, found: 339.0960 .

\subsection{Kinetic isotope effect studies}

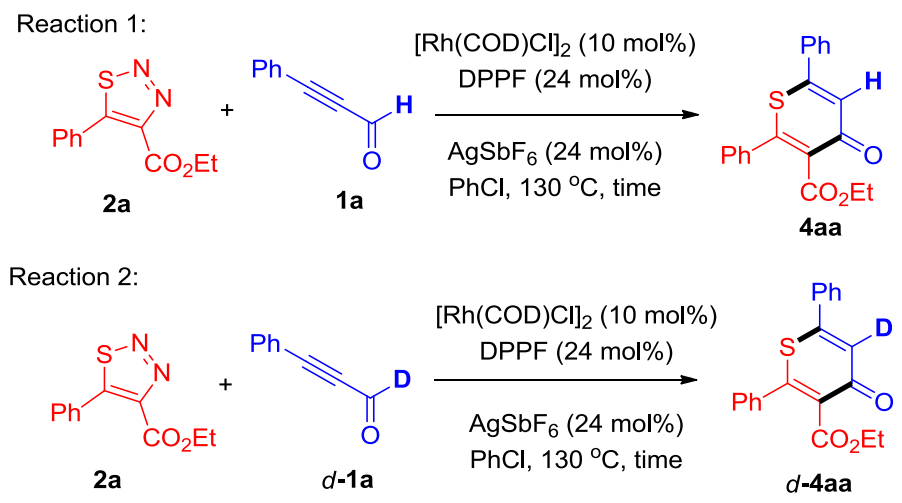

Scheme S15. Kinetic isotope effect studies

\section{Procedure for Kinetic isotope effect studies}

Two flame dried reaction tubes equipped with magnetic stirring bars were each charged with thiadiazole (2a, $24 \mathrm{mg}, 0.100 \mathrm{mmol}),\left[\mathrm{Rh}(\mathrm{COD}) \mathrm{Cl}_{2}\right.$ ( $\left.5 \mathrm{mg}, 0.010 \mathrm{mmol}\right)$, DPPF (14 mg, 0.024 mmol), $\mathrm{AgSbF}_{6}(9 \mathrm{mg}, 0.024 \mathrm{mmol})$, and internal standard 1,3,5- Trimethoxybenzene (17 mg, 0.100 $\mathrm{mmol})$, respectively. Then the reaction tubes were flushed with nitrogen. Ynal 1a (26 mg, 0.200 mmol) and $d$-1a (26 mg, $0.200 \mathrm{mmol})$ were dissolved in fresh distilled $\mathrm{PhCl}(1.5 \mathrm{~mL})$ and the solutions were added to the above two reaction tubes, respectively. The mixture were allowed to stir for $40 \mathrm{~min}$, then a small portion of the solution of the reaction 1 and reaction 2 were taken out via 
syringe for ${ }^{1} \mathrm{H}$ NMR analysis. Samples of $50 \mathrm{~min}, 60 \mathrm{~min}, 120 \mathrm{~min}, 180 \mathrm{~min}$ were also made by the same procedure.
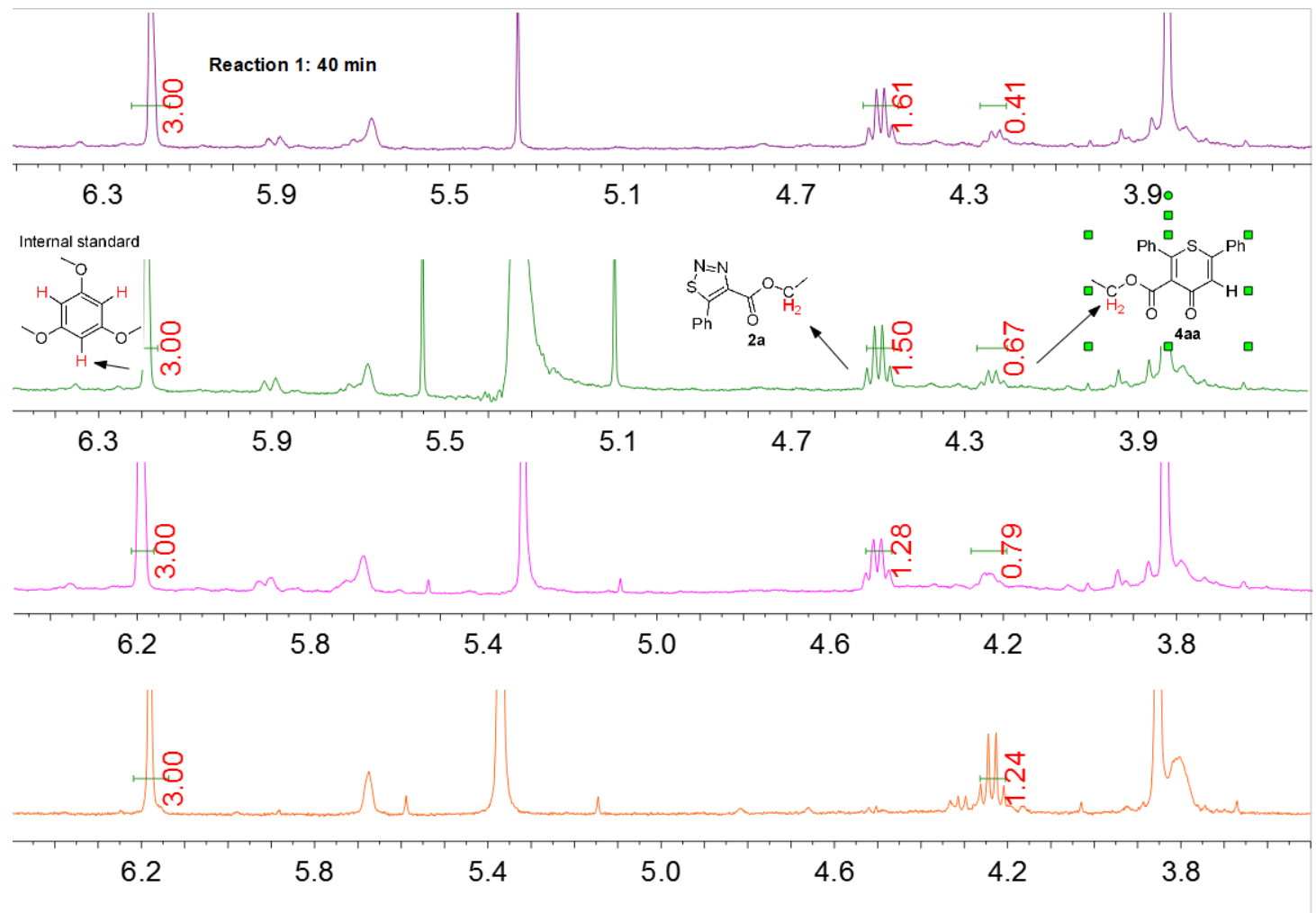

Figue S3. System ${ }^{1} \mathrm{H}$ NMR for reaction 1 


\section{Supporting Information}

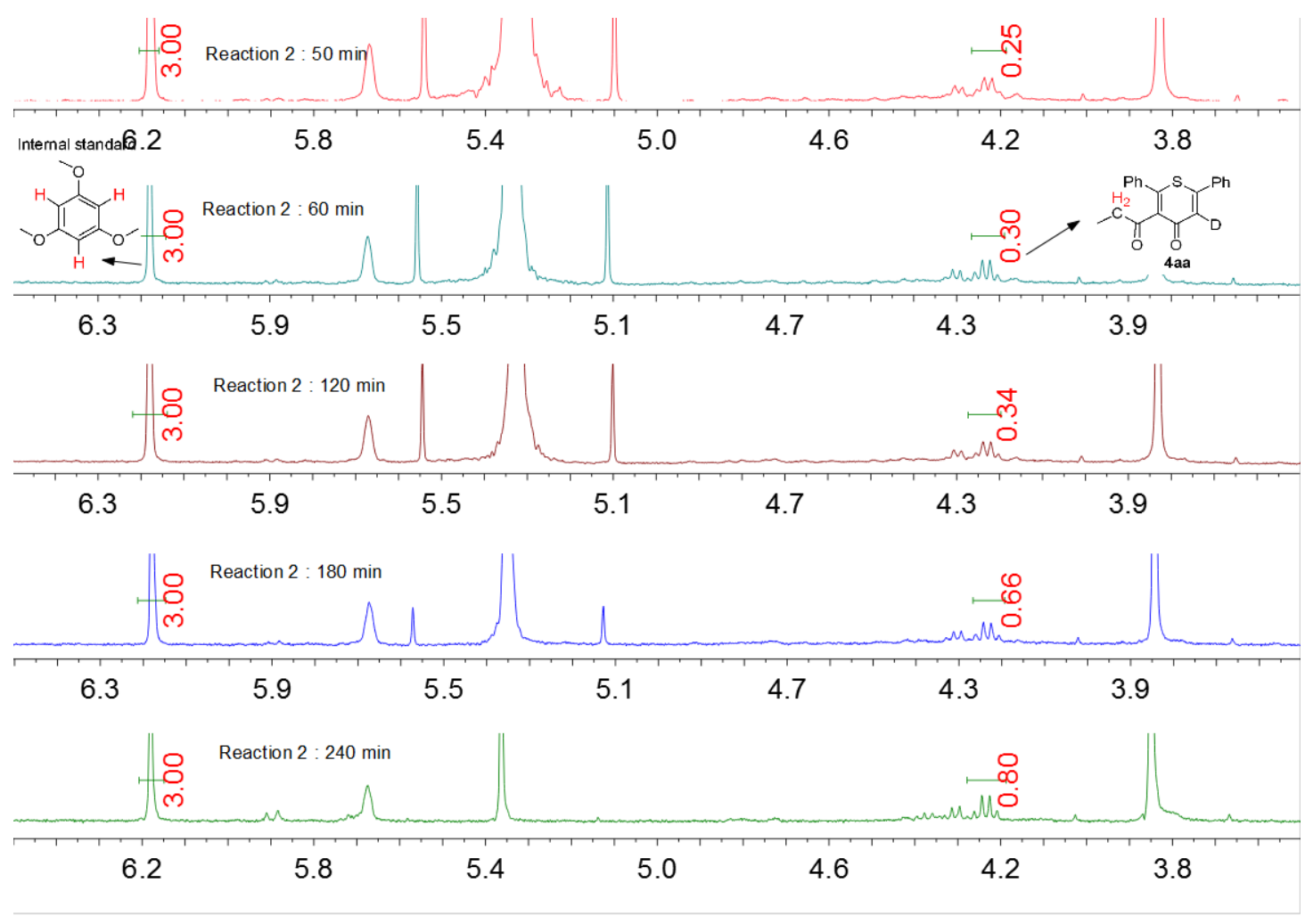

Figue S4. System ${ }^{1} \mathrm{H}$ NMR for reaction 2

Table S2. Yields of 4aa and $d$-4aa in the isotope effect study

\begin{tabular}{cccc}
\hline Entry & Time $(\mathrm{min})$ & Yield of $\mathbf{4 a a}(\%)$ & Yield of $d-\mathbf{4 a a}(\%)$ \\
\hline 1 & 20 & trace & trace \\
2 & 40 & 20.5 & 12.5 \\
3 & 50 & 33.5 & 15 \\
4 & 60 & 39.5 & 17 \\
5 & 120 & 62 & 33 \\
6 & 180 & - & 40 \\
\hline
\end{tabular}

\section{References}

[1] (a) Kurandina, D.; Gevorgyan, V. Rhodium Thiavinyl Carbenes from 1,2,3-Thiadiazoles Enable Modular Synthesis of Multisubstituted Thiophenes. Org. Lett. 2016, 18, 1804-1807. (b) Seo, B.; Kim, Y. G.; Lee, P. H. Synthesis of Isothiazole via the Rhodium-Catalyzed Transannulation of 1,2,3-Thiadiazoles with Nitriles. Org. Lett. 2016, 18, 5050-5053. (c) Son, J. -Y.; Kim, J.; Han, S. H.; Kim, S. H.; Lee, P. H. Regioselective Synthesis of Dihydrothiophenes and Thiophenes via the Rhodium-Catalyzed Transannulation of 1,2,3Thiadiazoles with Alkenes. Org. Lett. 2016, 18, 5408-5411.

[2] Zhou, Y.; Trewyn, B. G.; Angelici, R. J.; Woo, L. K. Catalytic Reactions of Carbene Precursors on Bulk Gold Metal. J. Am. Chem. Soc. 2009, 131, 11734-11743.

[3] (a) Tretyakov, E. V.; Tkachev, A. V.; Rybalova, T. V.; Gatilov, Y. V.; Knight, Vasilevsky, S. F. Reaction of Arylpropargyl Aldehydes with 2,3-Bis-hydroxylamino-2,3-dimethylbutane: 


\section{Supporting Information}

Synthesis of 2-(1-Hydroxy-4,4,5,5-tetramethylimidazolidin-2-ylidene)-1-arylethanones. Tetrahedron 2000, 56, 10075-10080. (b) Quesada, E.; Taylor, R. J. K. One-pot Conversion of Activated Alcohols into Terminal Alkynes Using Manganese Dioxide in Combination with the Bestmann-Ohira Reagent. Tetrahedron Lett. 2005, 46, 6473-6476. (c) Mahatthananchai, J.; Kaeobamrung, J.; Bode, J. W. Chiral $N$-Heterocyclic Carbene-Catalyzed Annulations of Enals and Ynals with Stable Enols: A Highly Enantioselective Coates-Claisen Rearrangement. ACS Catal. 2012, 2, 494-503. (d) Paioti, P. H. S.; Abboud, K. A.; Aponick, A. Catalytic Enantioselective Synthesis of Amino Skipped Diynes. J. Am. Chem. Soc. 2016, 138, 2150 2153.

[4] (a) Journet, M.; Cai, D.; DiMichele, L. M.; Larsen, R. D. Highly Efficient Synthesis of $\alpha, \beta-$ Acetylenic Aldehydes from Terminal Alkynes using DMF as the Formylating Reagent. Tetrahedron Lett. 1998, 39, 6427-6428. (b) Guan, Y.; López-Alberca, M. P.; Lu, Z.; Zhang, Y.; Desai, A. A.; Patwardhan, A. P.; Dai, Y.; Vetticatt, M. J.; Wulff, W. D. Catalytic Asymmetric Synthesis of Alkynyl Aziridines: Both Enantiomers of cis-Aziridines from One Enantiomer of the Catalyst. Chem. Eur. J. 2014, 20, 13894-13900. (c) Kanakam, C. C.; Mani, N. S.; Ramanathan, H.; Rao, G. S. R. S. Syntheses Based on Cyclohexadienes. Part 2. Convenient Synthesis of 6-Alkylsalicylates, 6-Alkyl-2,4-dihydroxybenzoate, and 2,5Dialkylresorcinols. J. Chem. Soc. Perkin Trans. I 1989, 1907-1913. (d) Calderone J. A. and Santos, W. L. Copper(II)-Catalyzed Silylation of Activated Alkynes in Water: Diastereodivergent Access to $E$ - or Z- $\beta$-Silyl- $\alpha, \beta-$ Unsaturated Carbonyl and Carboxyl Compounds. Angew. Chem., Int. Ed. 2014, 53, 4154-4158. (e) Xia, Y.; Ge, R.; Chen, L.; Liu, Z.; Xiao, Q.; Zhang, Y.; Wang, J. Palladium-Catalyzed Oxidative Cross-Coupling of Conjugated Enynones with Organoboronic Acids. J. Org. Chem. 2015, 80, 7856-7864.

[5] (a) Golovanov, A. A.; Latypova, D. R.; Bekin, V. V.; Pisareava, V. S.; Vologzhanina, A. V.; Dokichev, V. A. Synthesis of 1,5-Disubstituted (E)-pent-2-en-4-yn-1-ones. Russian. J. Org. Chem. 2013, 49, 1264-1269. (b) Zheng, J.; Li, P.; Gu, M.; Lin, A.; Yao, H. Synthesis of Spiropentadiene Pyrazolones by $\mathrm{Rh}(\mathrm{III})$-Catalyzed Formal $\mathrm{sp}^{3} \mathrm{C}-\mathrm{H}$ Activation/Annulation. Org. Lett. 2017, 19, 2829-2832. (c) Wadsworth, D. H.; Geer, S. M.; Detty, M. R. Preparation of Arylpropiolate Esters from Trichlorocyclopropenium Cation and Elaboration of the Esters to Unsymmetrical 1,4-Pentadiyn-3-ones and Unsymmetrical Tellurapyranones. J. Org. Chem. 1987, 52, 3662-3668. (d) Song, B.; Li, L. -H.; Song, X. -R.; Qiu, Y. -F.; Zhong, M. -J.; Zhou, P. -X.; Liang, Y. -M. Zinc-Catalyzed [4+3] Cycloaddition with Concomitant Furan Annulation: Formation of Cyclohepta[b]Furans. Chem. Eur. J. 2014, 20, 5910-5913. (e) Wei, W.; Hamamoto, Y.; Ukaji, Y.; Inomata, K. Asymmetric Addition of Phenylzinc Reagents to $C$ Alkynyl Nitrones. Enantiomeric Enhancement by a Product-like Additive. Tetrahedron: Asymmetry. 2008, 19, 476-481. (f) Solorio-Alvarado, C. R.; Echavarren, A. M. GoldCatalyzed Annulation/Fragmentation: Formation of Free Gold Carbenes by RetroCyclopropanation. J. Am. Chem. Soc. 2010, 132, 11881-11883. (g) Kaya, U.; Chauhan, P.; Hack, D.; Deckers, K.; Puttreddy, R.; Rissanenb, K.; Enders, D. Enantioselective Synthesis of 4H-Pyranonaphthoquinones via Sequential Squaramide and Silver Catalysis. Chem. Commun. 2016, 52, 1669-1672. (h) Fang, J. -K.; An, D. -L.; Wakamatsu, K.; Ishikawa, T.; Iwanaga, T.; Toyota, S.; Akita, S. -I.; Matsuo, D.; Orita, A.; Otera, J. Synthesis and Spectroscopic Study of Phenylenee(poly)ethynylenes Substituted by Amino or Amino/Cyano Groups at Terminal(s): Electronic Effect of Cyano Group on Charge-transfer Excitation of Acetylenic $\pi$-Systems. 


\section{Supporting Information}

Tetrahedron 2010, 66, 5479-5485. (i) Nowak-Król, A.; Koszarna, B.; Yoo, S. Y.; Chromiński, J.; Węcławski, M. K., Lee, C. -H.; Gryko, D. T. Synthesis of trans- $\mathrm{A}_{2} \mathrm{~B}_{2}$-Porphyrins Bearing Phenylethynyl Substituents. J. Org. Chem. 2011, 76, 2627-2634.

[6] (a) Brindani, N.; Rassu, G.; Dell'Amico, L.; Zambrano, V.; Pinna, L.; Curti, C.; Sartori, A.; Battistini, L.; Casiraghi, G.; Pelosi, G.; Greco, D.; Zanardi, F. Organocatalytic, Asymmetric Eliminative [4+2] Cycloaddition of Allylidene Malononitriles with Enals: Rapid Entry to Cyclohexadiene-Embedding Linear and Angular Polycycles. Angew. Chem., Int. Ed. 2015, 54, 7386-7390. (b) Landa, A.; Puente, Á.; Santos, J. I.; Vera, S.; Oiarbide, M.; Palomo, C. Catalytic Conjugate Additions of Geminal Bis(sulfone)s: Expanding the Chemistry of Sulfones as Simple Alkyl Anion Equivalents. Chem. Eur. J. 2009, 15, 11954-11962. (c) Wickham, G.; Wells, G. J.; Waykole, L.; Paquette, L. A. Strain-Activated 1,3-Butadienes. Synthesis and Dienic Reactivity of Dicyclobutylideneethane. J. Org. Chem. 1985, 50, 3485-3489. (d) Pettigrew, J. D.; Cadieux, J. A.; So, S. S. S.; Wilson, P. D. Phenylboronic Acid Mediated Triple Condensation Reactions of Phloroglucinol and Unsaturated Carbonyl Compounds. Org. Lett. 2005, 7, 467-470. (e) Petroski, R. J.; Vermillion, K;. Cossé, A. A. Two-Carbon Homologation of Aldehydes and Ketones to $\alpha, \beta$-Unsaturated Aldehydes. Molecules 2011, 16, 5062-5078. (f) Gupta, S.; Xie, P.; Xia, Y.; Lee, D. Reactivity of Arynes toward Functionalized Alkenes: Intermolecular Alder-ene vs. Addition Reactions. Org. Chem. Front. 2018, 5, 2208-2213. (g) Raya, B.; Jing, S.; Balasanthiran, V.; RajanBabu, T. V. Control of Selectivity through Synergy between Catalysts, Silanes, and Reaction Conditions in Cobalt-Catalyzed Hydrosilylation of Dienes and Terminal Alkenes. ACS Catal, 2017, 7, 2275-2283.

[7] (a) Zhu, S.; Wang, C.; Chen, L.; Liang, R.; Yu, Y.; Jiang, H. Modular Approach for Synthesis of Vicinal Diamines Containing Axial Chiral 1,1'-Binaphthyl from 1,2-Diaminoethane by PdCatalyzed N-Arylation Reactions. Org. Lett. 2011, 13, 1146-1149. (b) Chen, Q. -Y.; Yang, Z. Y. Palladium-Catalyzed Reaction of Phenyl Fluoroalkanesulfonates with Alkynes and Alkenes. Tetrahedron Lett. 1986, 27, 1171-1174. (c) Xu, Z. -F.; Dai, H.; Shan, L.; Li, C. -Y. Metal-Free Synthesis of $(E)$-Monofluoroenamine from 1-Sulfonyl-1,2,3-Triazole and $\mathrm{Et}_{2} \mathrm{O} \cdot \mathrm{BF}_{3}$ via Stereospecific Fluorination of $\alpha$-Diazoimine. Org. Lett. 2018, 20, 1054-1057.

[8] (a) Chen, F.; Lai, S. -Q.; Zhu, F. -F.; Meng, Q.; Jiang, Y.; Yu, W.; Han, B. Cu-Catalyzed Radical Cascade Annulations of Alkyne-Tethered $N$-Alkoxyamides with Air: Facile Access to Isoxazolidine/1,2-Oxazinane-Fused Isoquinolin-1(2H)-ones. ACS Catal. 2018, 8, 8925-8931. (b) Ayan, D.; Maltais, R.; Hospital, A.; Poirier, D. Chemical Synthesis, Cytotoxicity, Selectivity and Bioavailability of $5 \alpha$-Androstane-3 $\alpha, 17 \beta$-Diol Derivatives. Bioorg. Med. Chem. 2014, 22, $5847-5859$.

[9] Zhao, H. -Y.; Wu, F. -S.; Yang, L.; Liang, Y.; Cao, X. -L.; Wang, H. -S.; Pan, Y. -M. Catalystand Solvent-Free Approach to 2-Arylated Quinolines via [5+1] Annulation of 2Methylquinolines with Diynones. RSC Adv. 2018, 8, 4584-4587.

[10] (a) Vallet, A.; Janin, A.; Romanet, R. Préparation de composés acétyléniques deutériés IV Obtention d'aldéhydes $\alpha$-acétyléniques deutériés sur la fonction aldéhyd. J. Labelled Compd. Rad. 1970, 7, 80-83. (b) Verlee, A.; Heugebaert, T.; Meer, T. V. D.; Kerchev, P.; Breusegem, F. V.; Stevens, C. V. Domino Reaction of a Gold Catalyzed 5-endo-dig Cyclization and a [3,3]Sigmatropic Rearrangement towards Polysubstituted Pyrazoles. Org. Biomol. Chem. 2018, 16, 9359-9363. 
9. Copies of NMR Spectra of Products and Materials

${ }^{1} \mathrm{H}$ and ${ }^{13} \mathrm{C}$ of compound $4 \mathrm{aa}$ in $\mathrm{CDCl}_{3}$

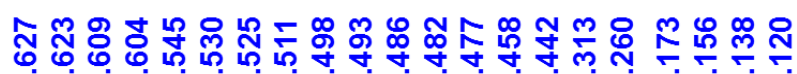

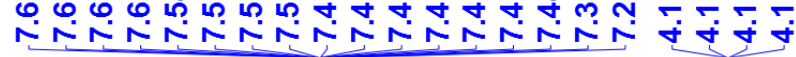

苦产家

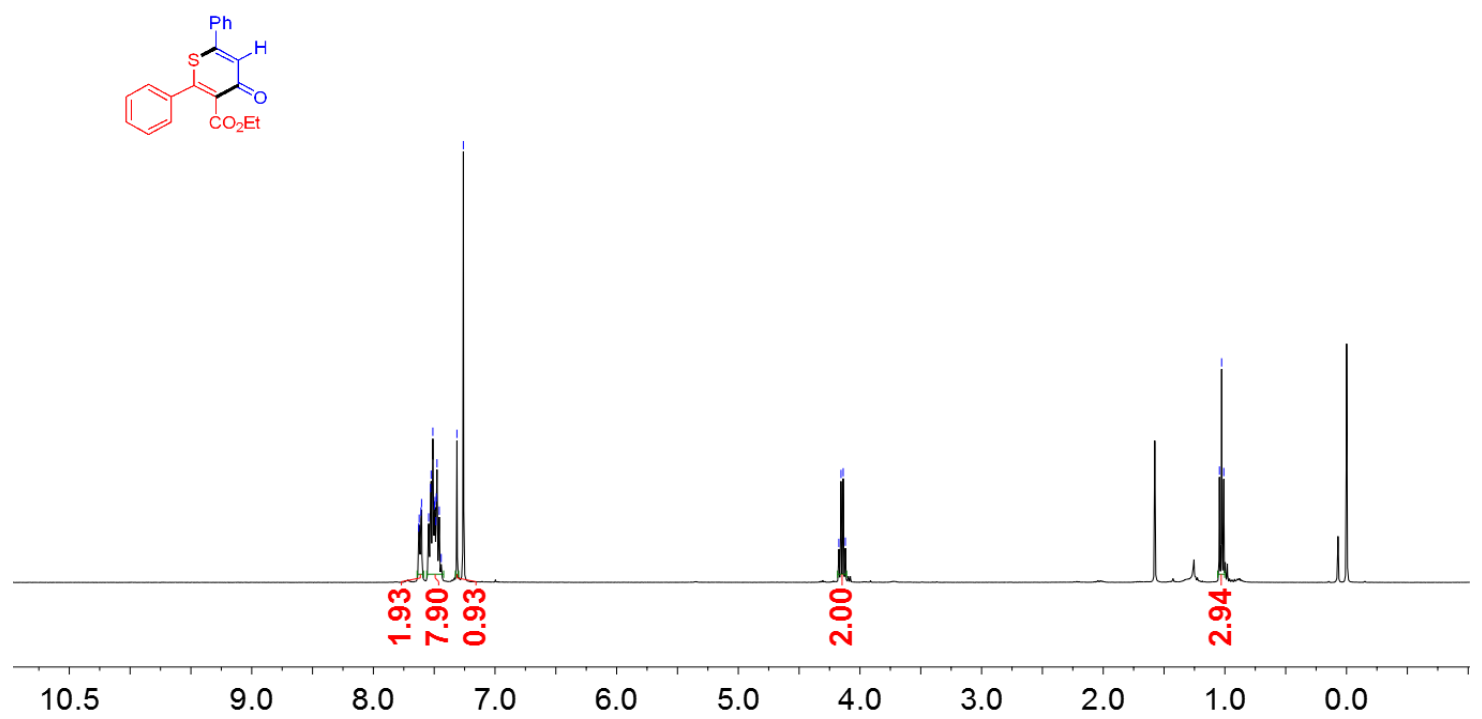

帝

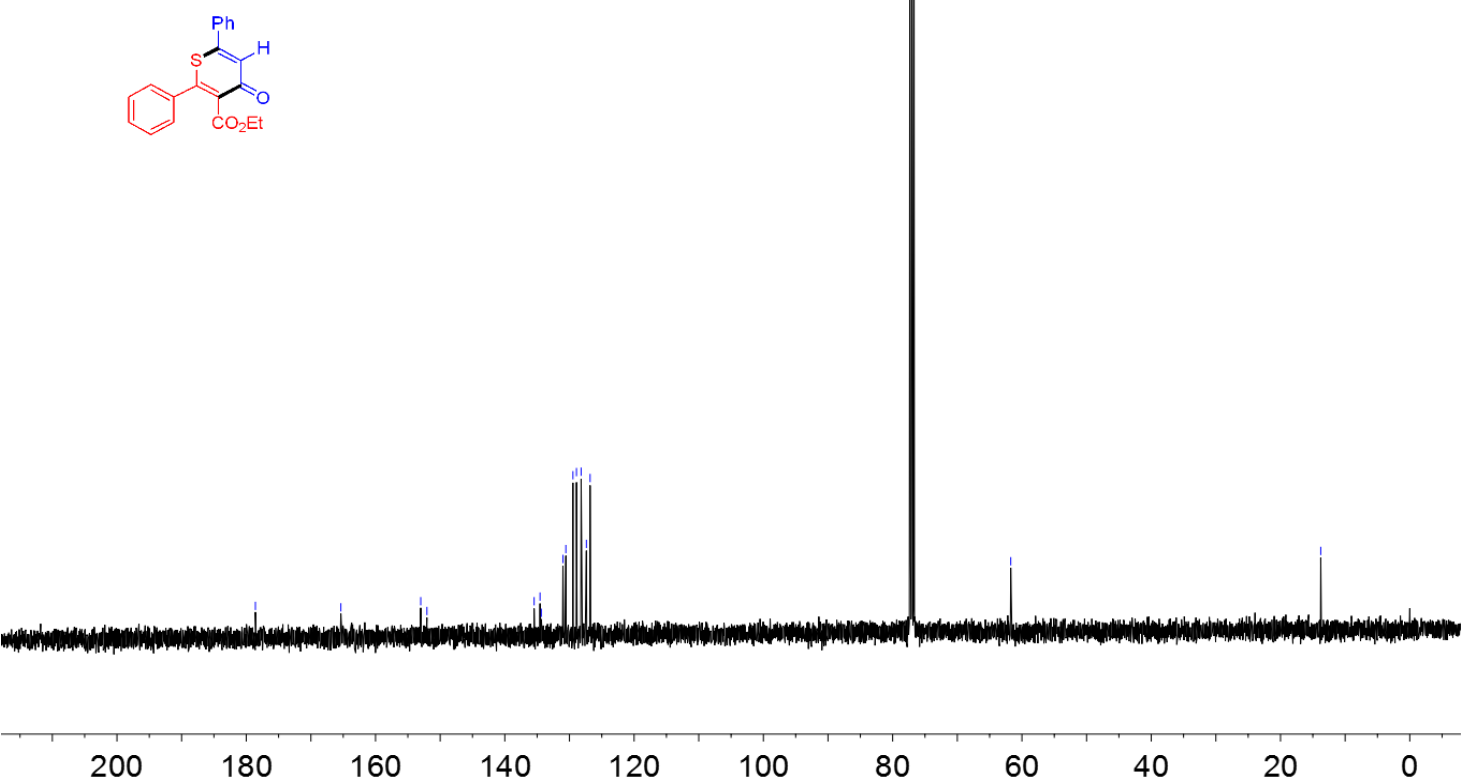


${ }^{1} \mathrm{H}$ and ${ }^{13} \mathrm{C}$ of compound $4 \mathrm{ab}$ in $\mathrm{CDCl}_{3}$

กิ స్లి

m

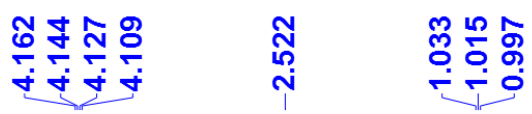
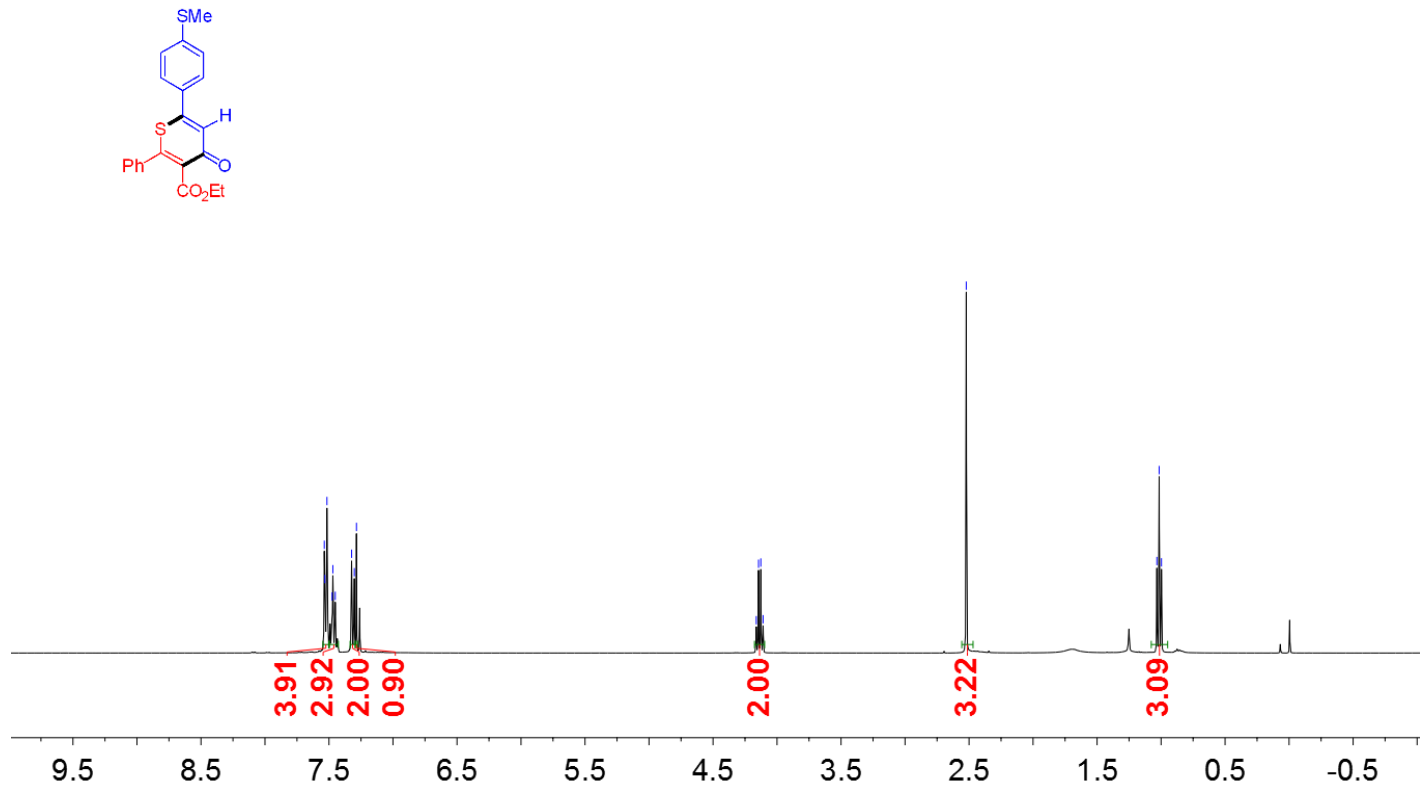

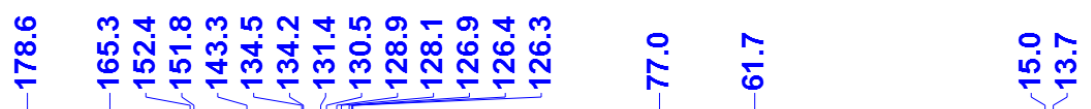
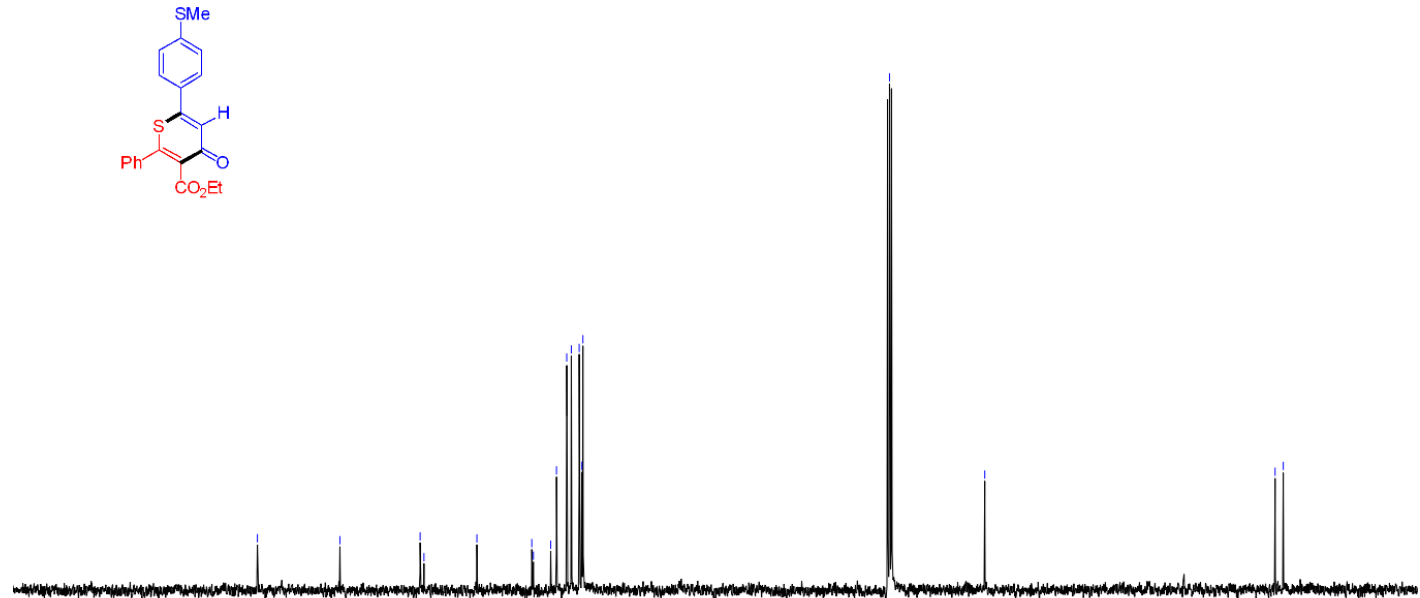
${ }^{1} \mathrm{H}$ and ${ }^{13} \mathrm{C}$ of compound $4 \mathrm{ac}$ in $\mathrm{CDCl}_{3}$

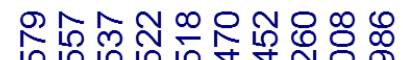

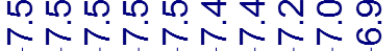

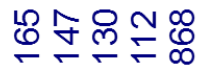

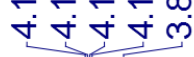

लำ

-
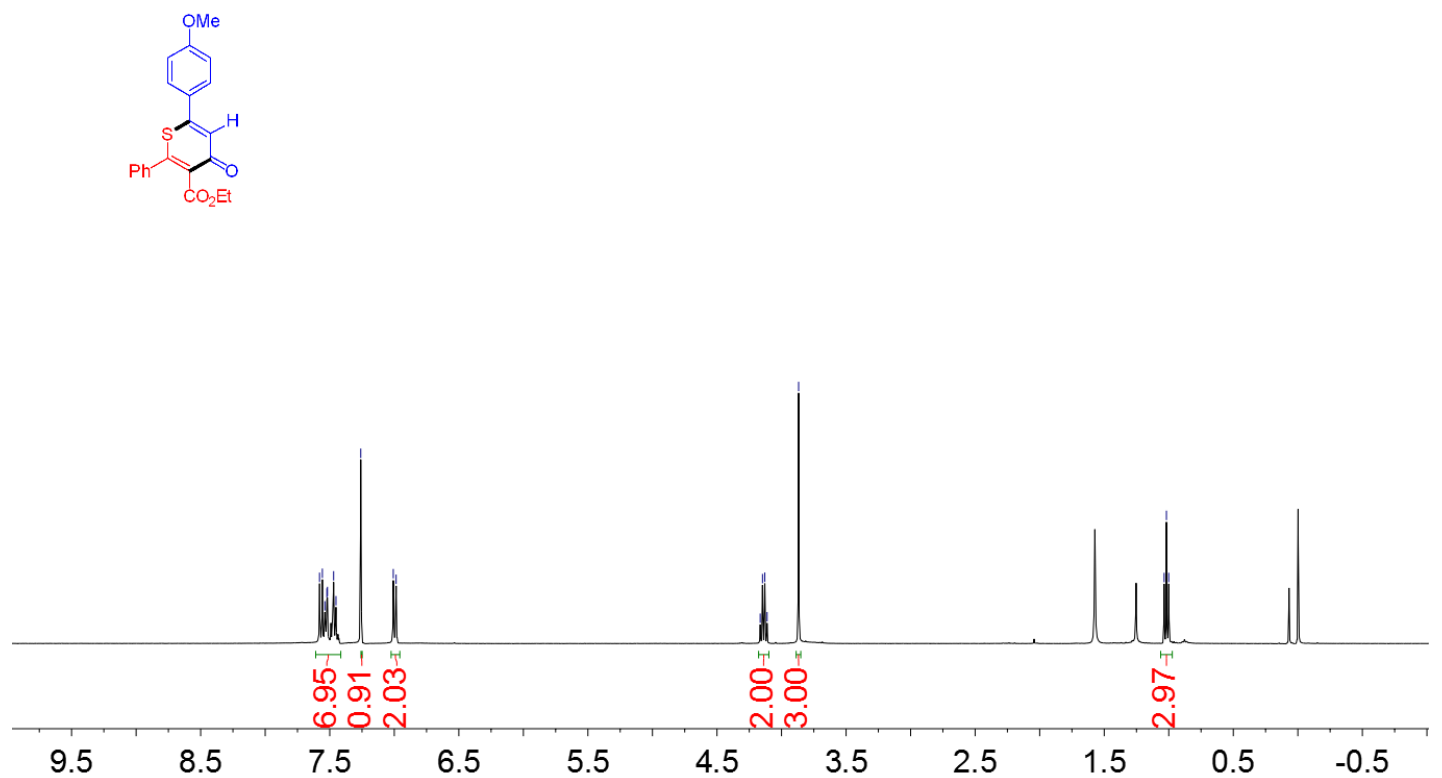

ก

ఎ

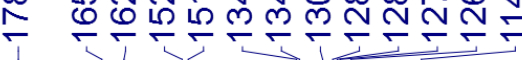

슨

$\overline{6} \stackrel{\circ}{\circ}$

$\stackrel{\sim}{\tilde{m}}$
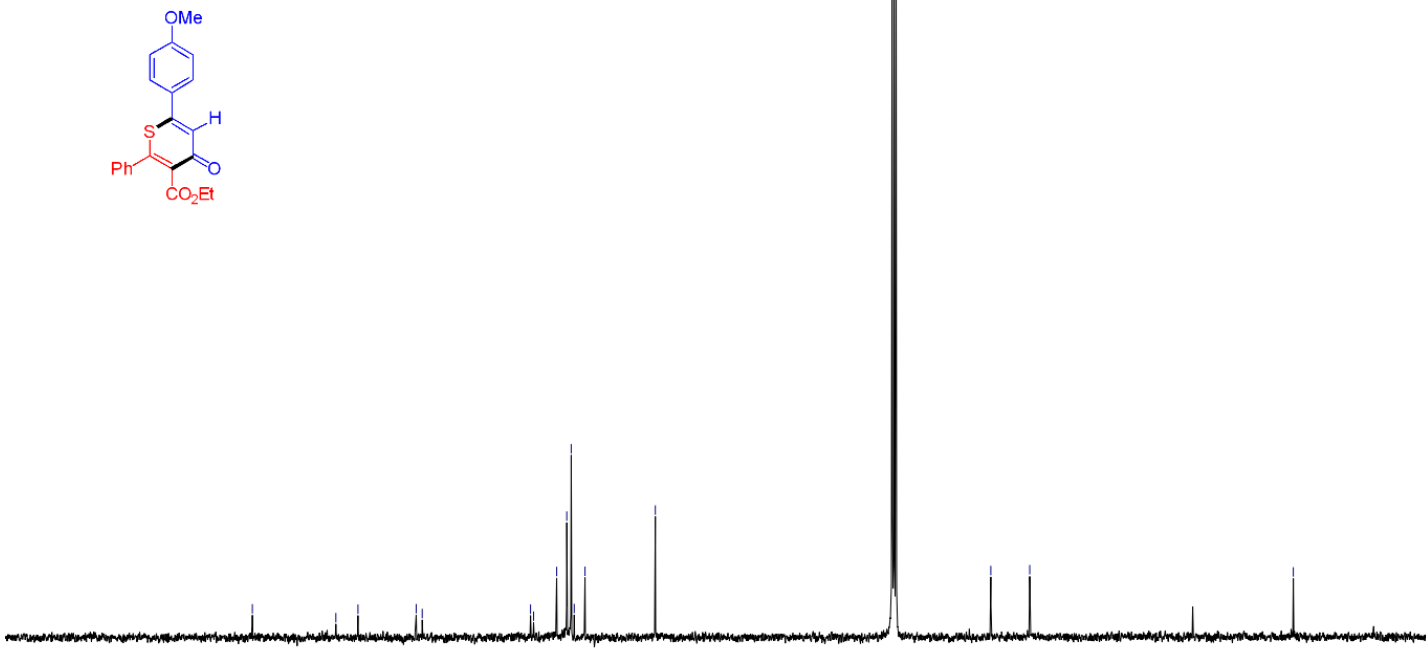

200

180

160

140

120

100

80

60

40 
${ }^{1} \mathrm{H}$ and ${ }^{13} \mathrm{C}$ of compound $4 \mathrm{ad}$ in $\mathrm{CDCl}_{3}$

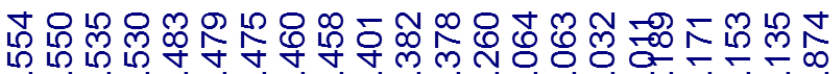

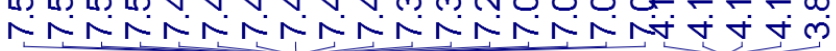

ฺֻู

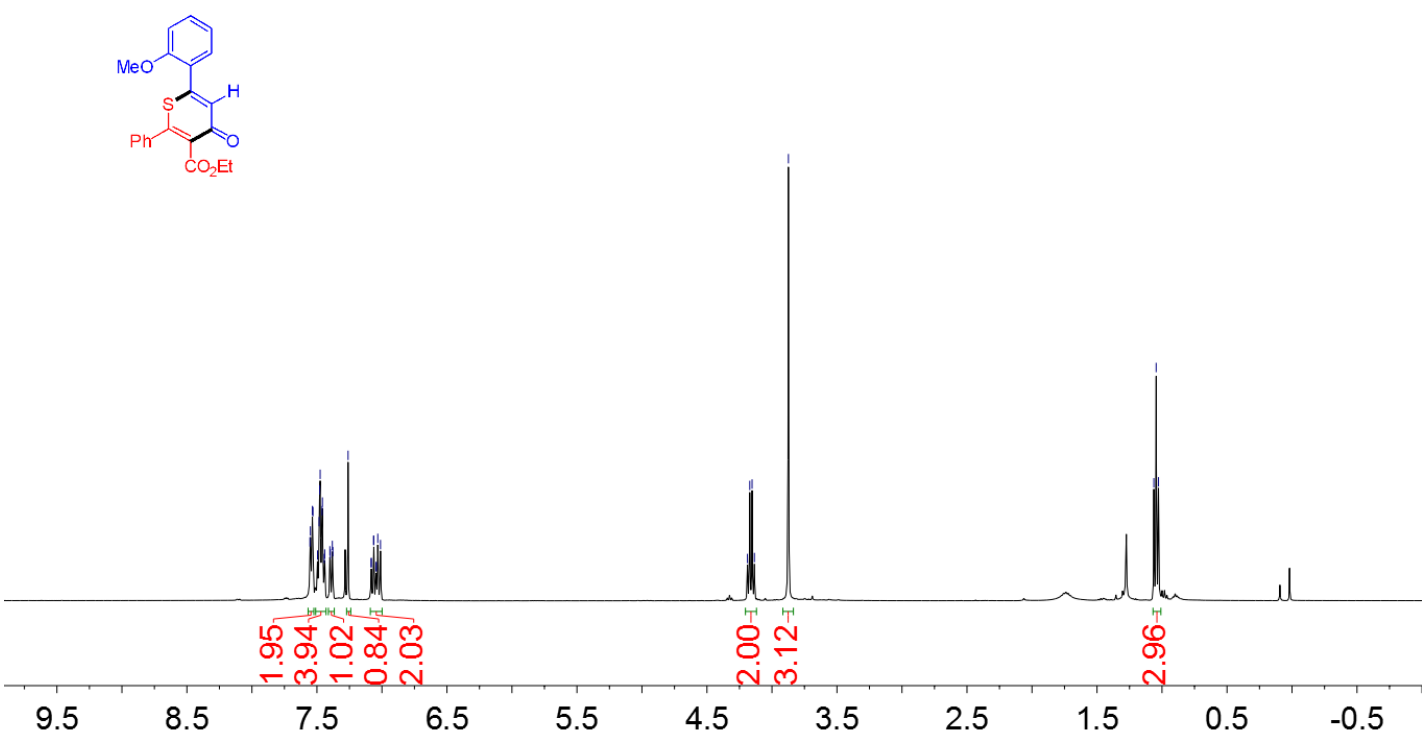

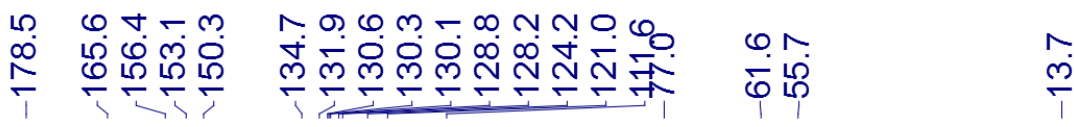
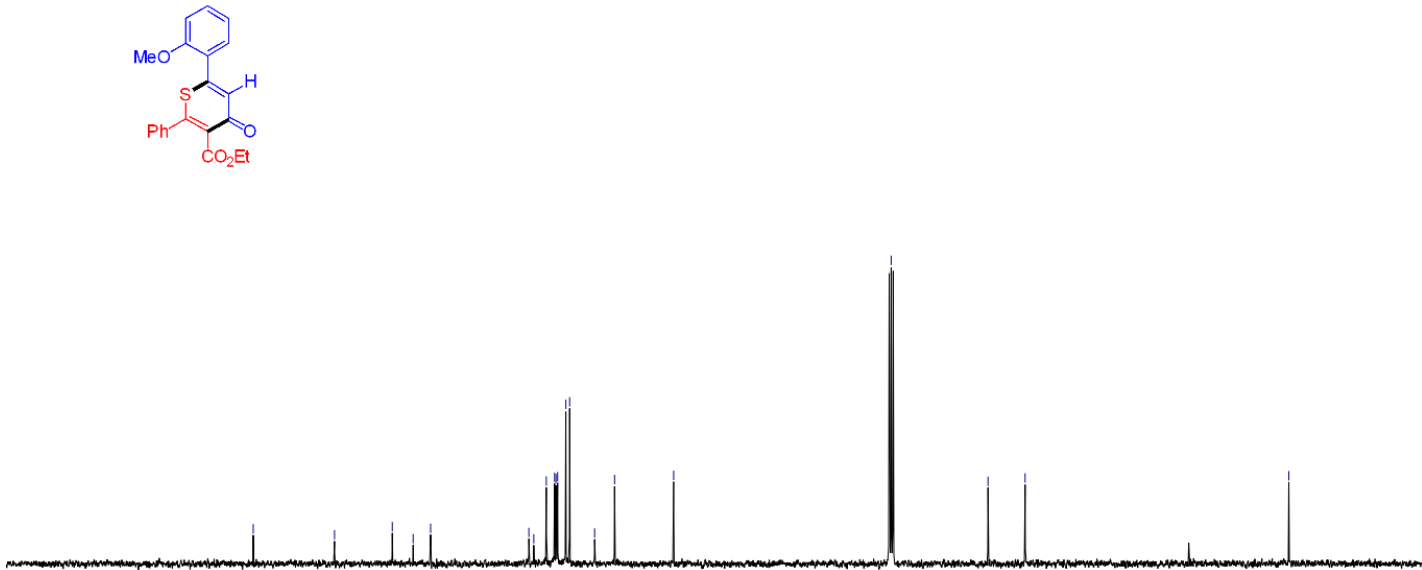
${ }^{1} \mathrm{H}$ and ${ }^{13} \mathrm{C}$ of compound $4 \mathrm{ae}$ in $\mathrm{CDCl}_{3}$

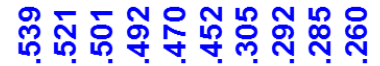

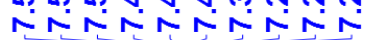

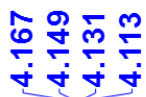

糵

홍

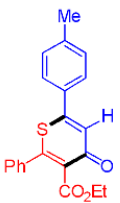

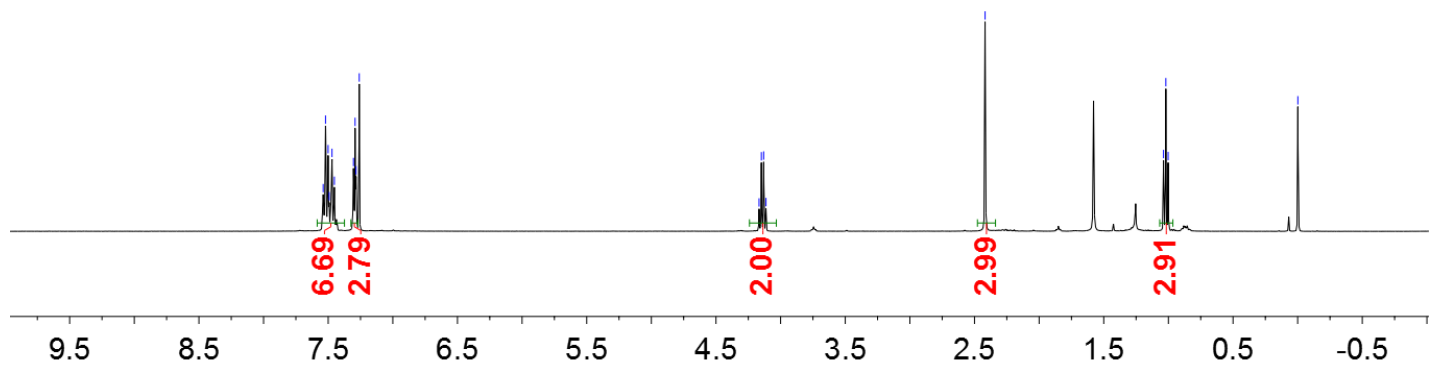

ก 它

$\$$

$\stackrel{\nabla}{\bar{\top}} \stackrel{\Gamma}{\Gamma}$
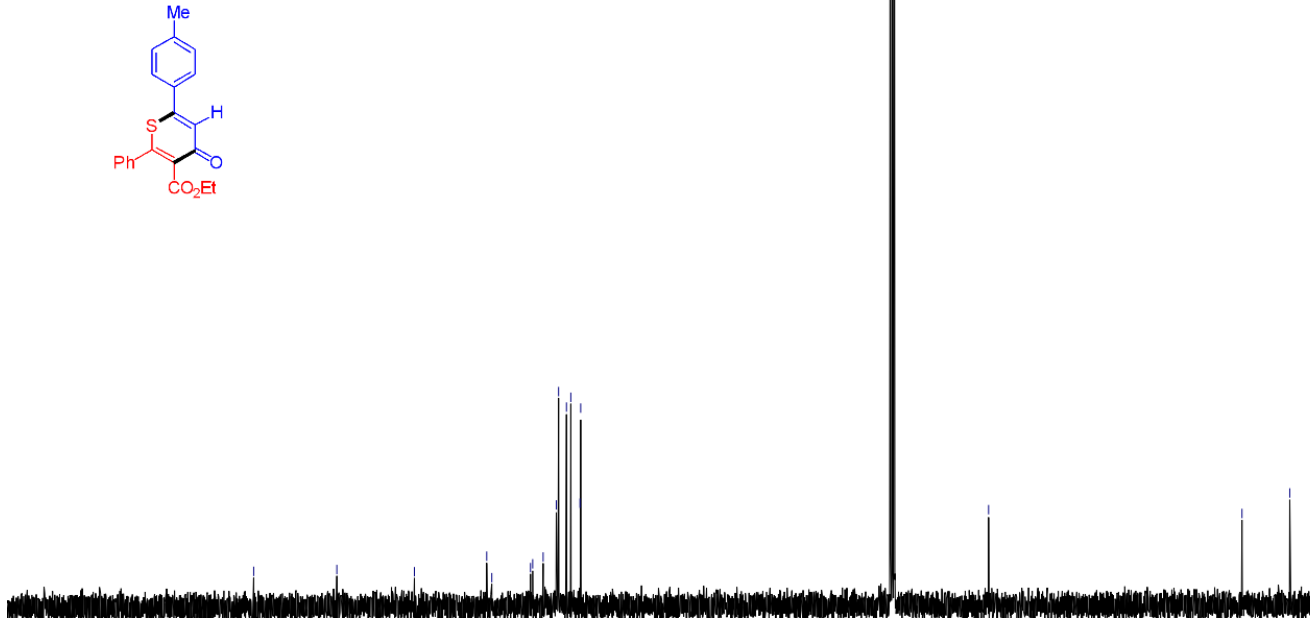
${ }^{1} \mathrm{H}{ }^{13} \mathrm{C}$ and ${ }^{19} \mathrm{~F}$ of compound 4 af in $\mathrm{CDCl}_{3}$

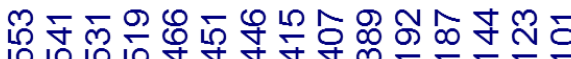
NNNNNNNNNNNN ㄸํㅇํㅇ
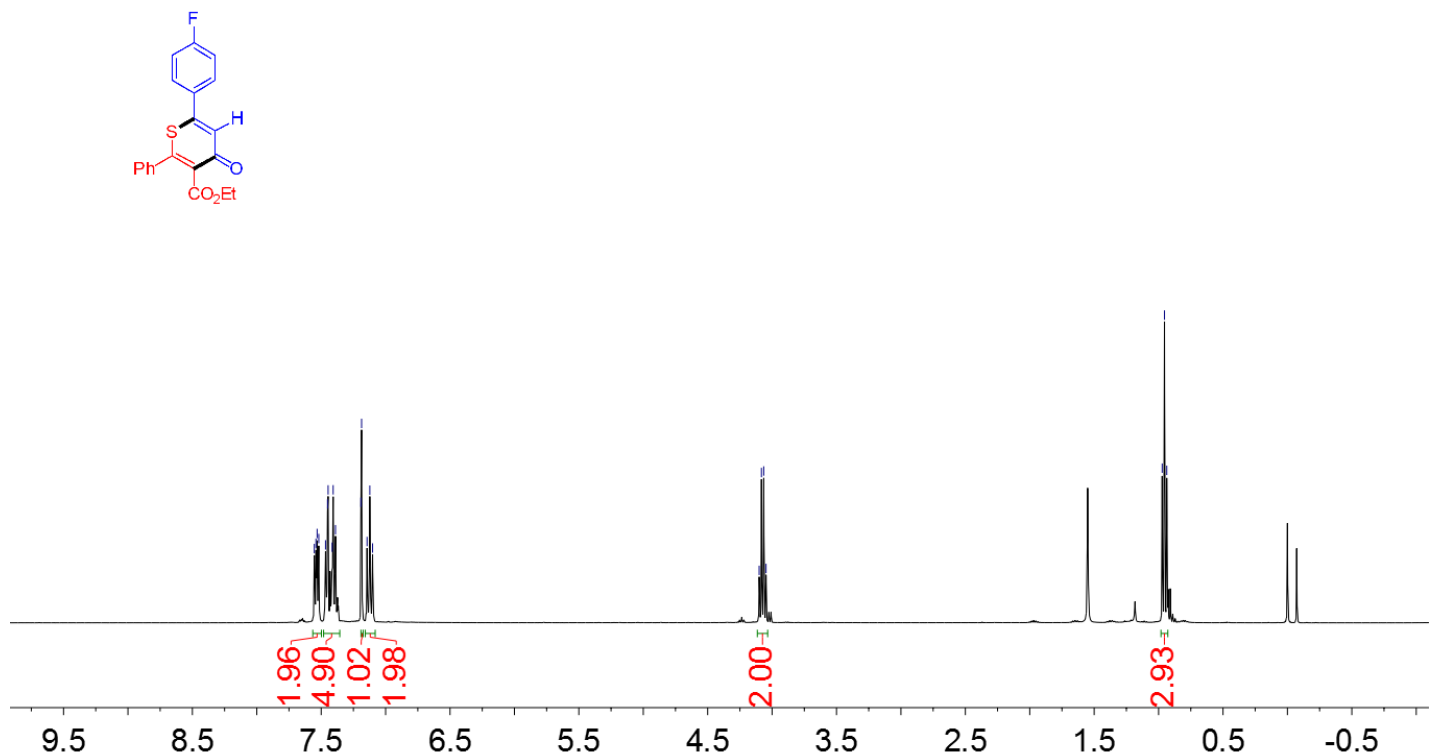

L

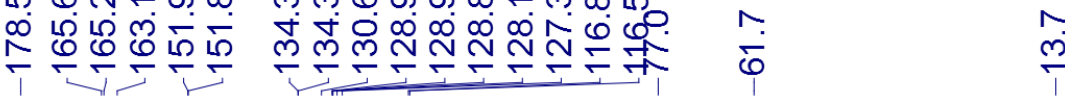

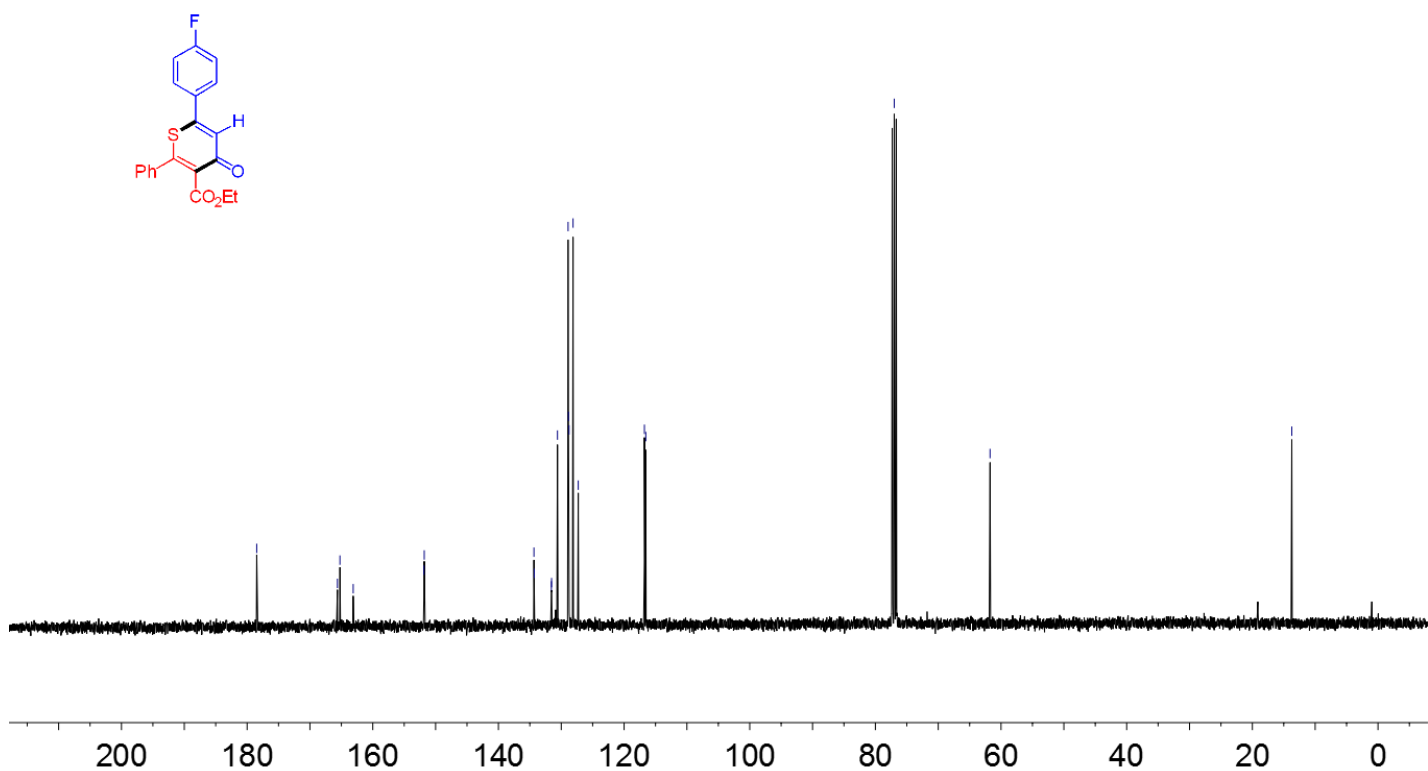




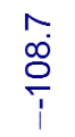
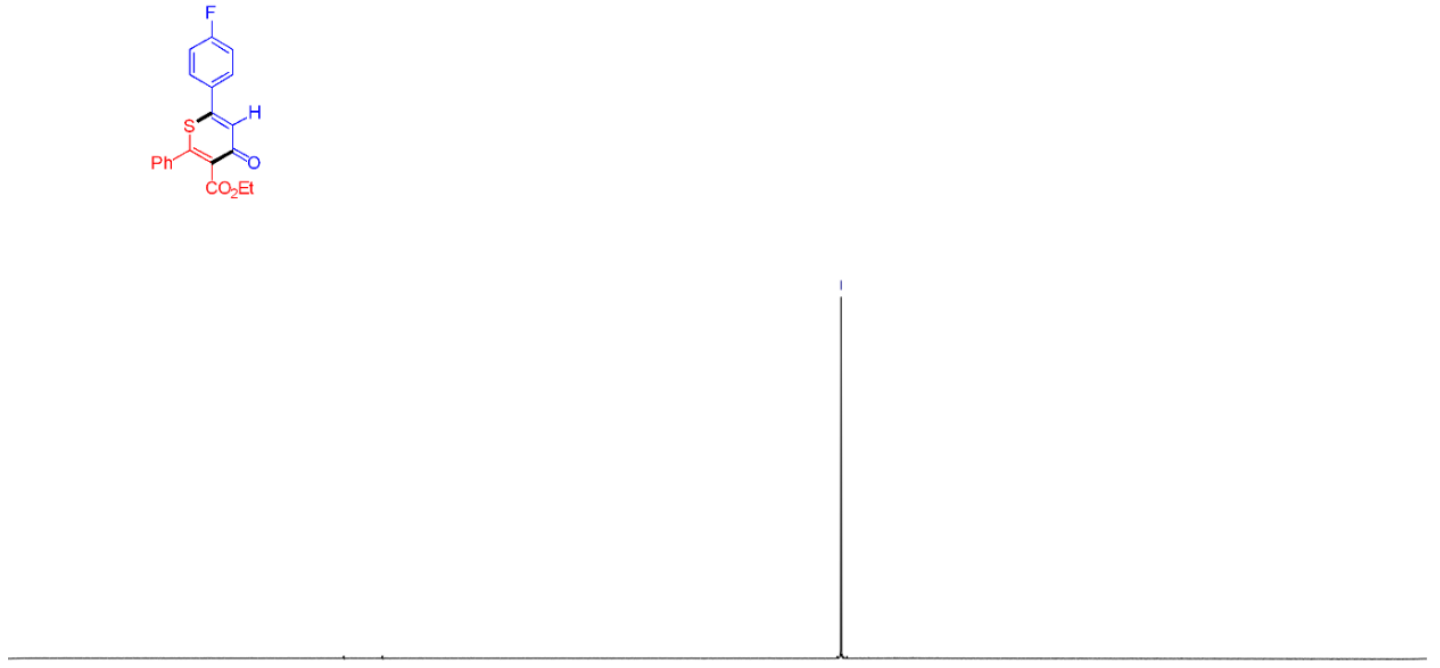
${ }^{1} \mathrm{H}$ and ${ }^{13} \mathrm{C}$ of compound $4 \mathrm{ag}$ in $\mathrm{CDCl}_{3}$

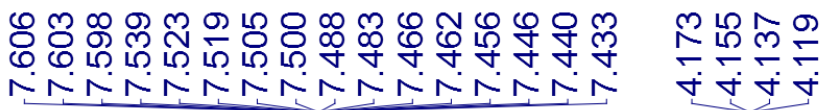

요옹음

开
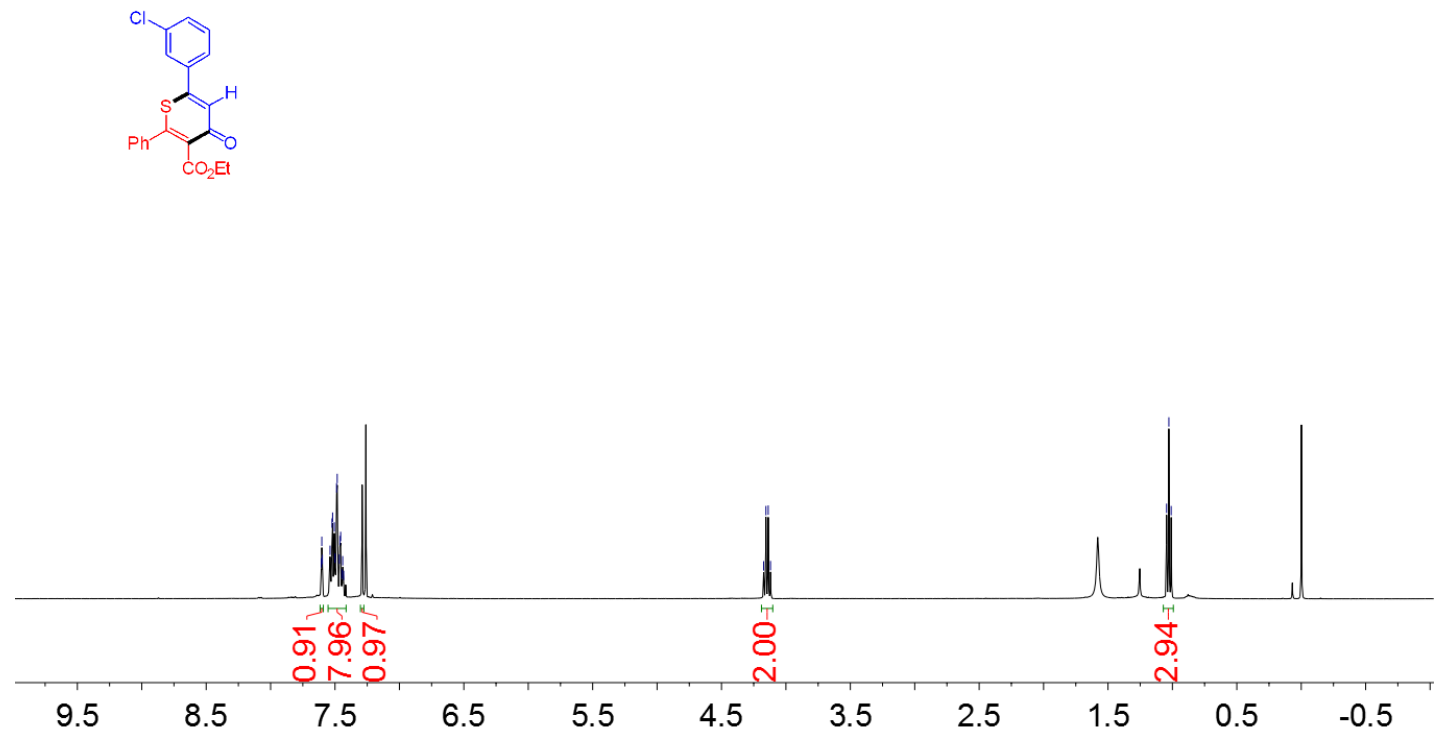

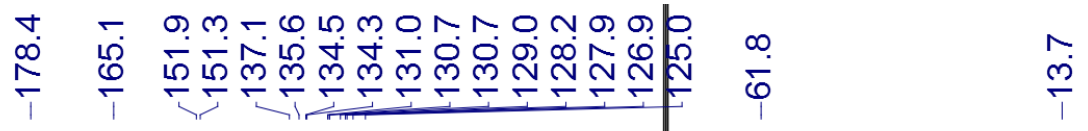<smiles>Oc1cccc(-c2ccccc2)c1</smiles>

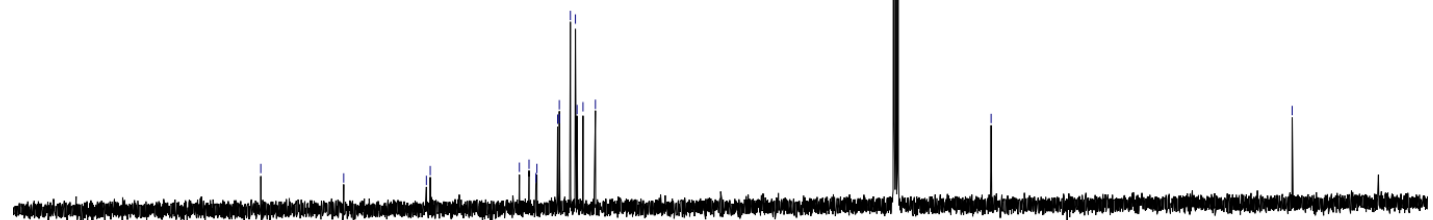

200

180

160

140

120

100

80

60

40

20 
${ }^{1} \mathrm{H}$ and ${ }^{13} \mathrm{C}$ of compound $4 \mathrm{ah}$ in $\mathrm{CDCl}_{3}$

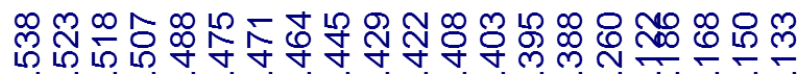

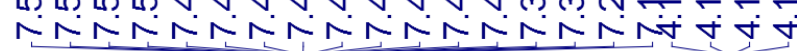
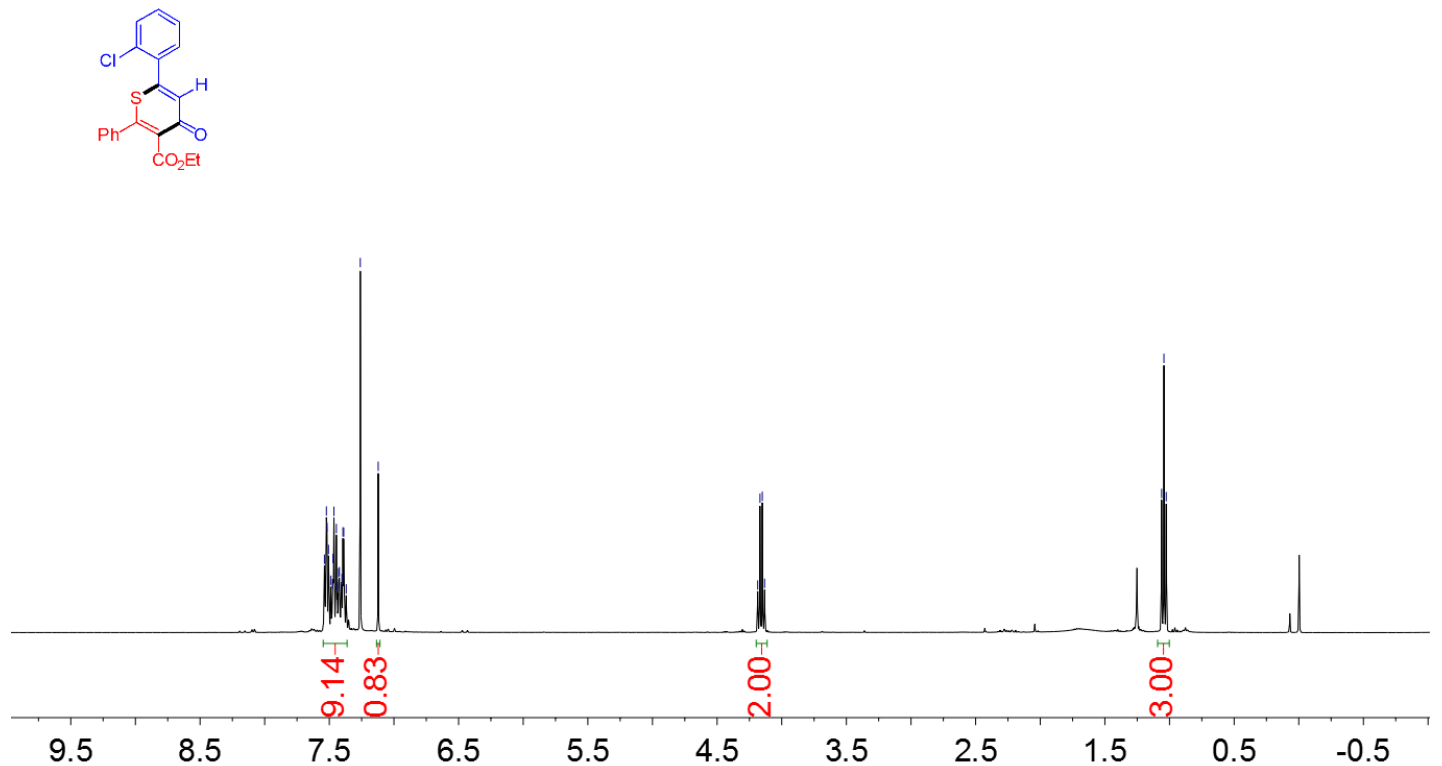

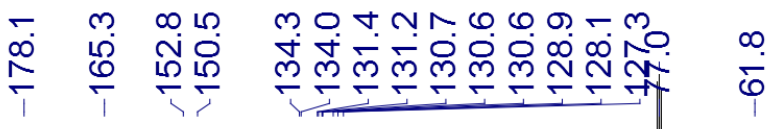

$\stackrel{\infty}{m}$
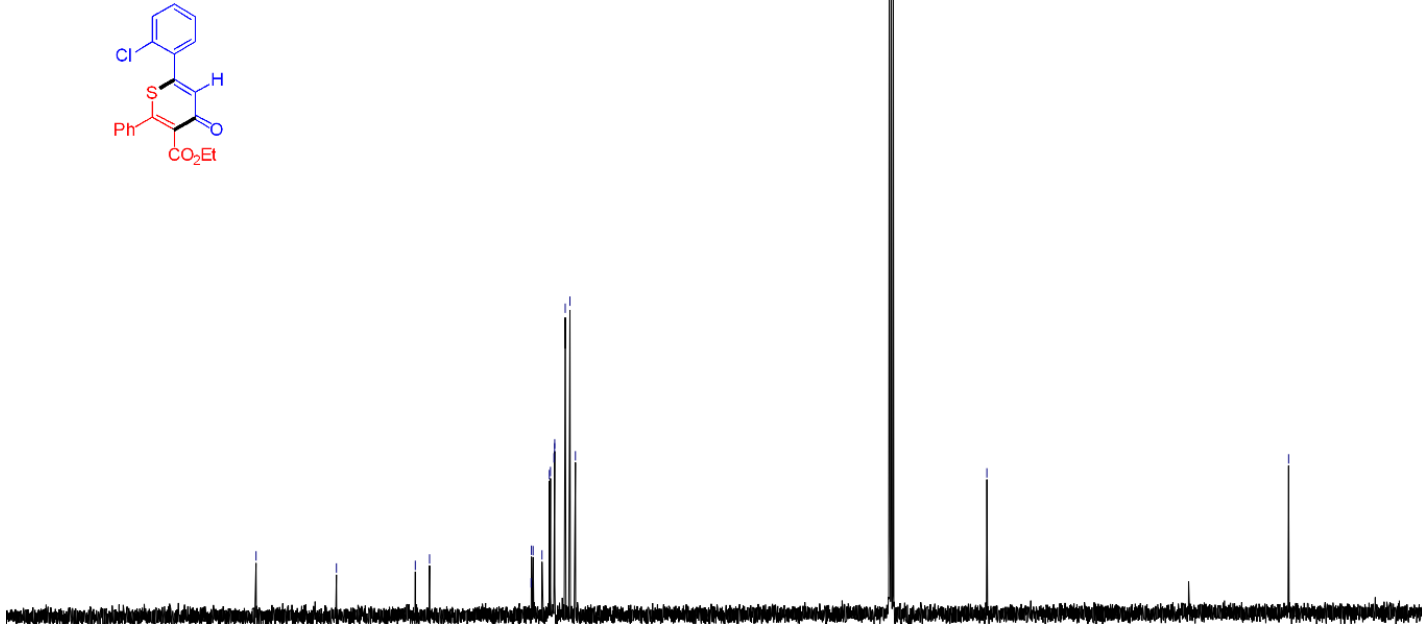

200

180

160

140

120

100

80

60

40

20 
${ }^{1} \mathrm{H}$ and ${ }^{13} \mathrm{C}$ of compound 4 ai in $\mathrm{CDCl}_{3}$

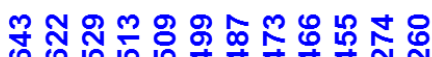

㲅守导守安

象
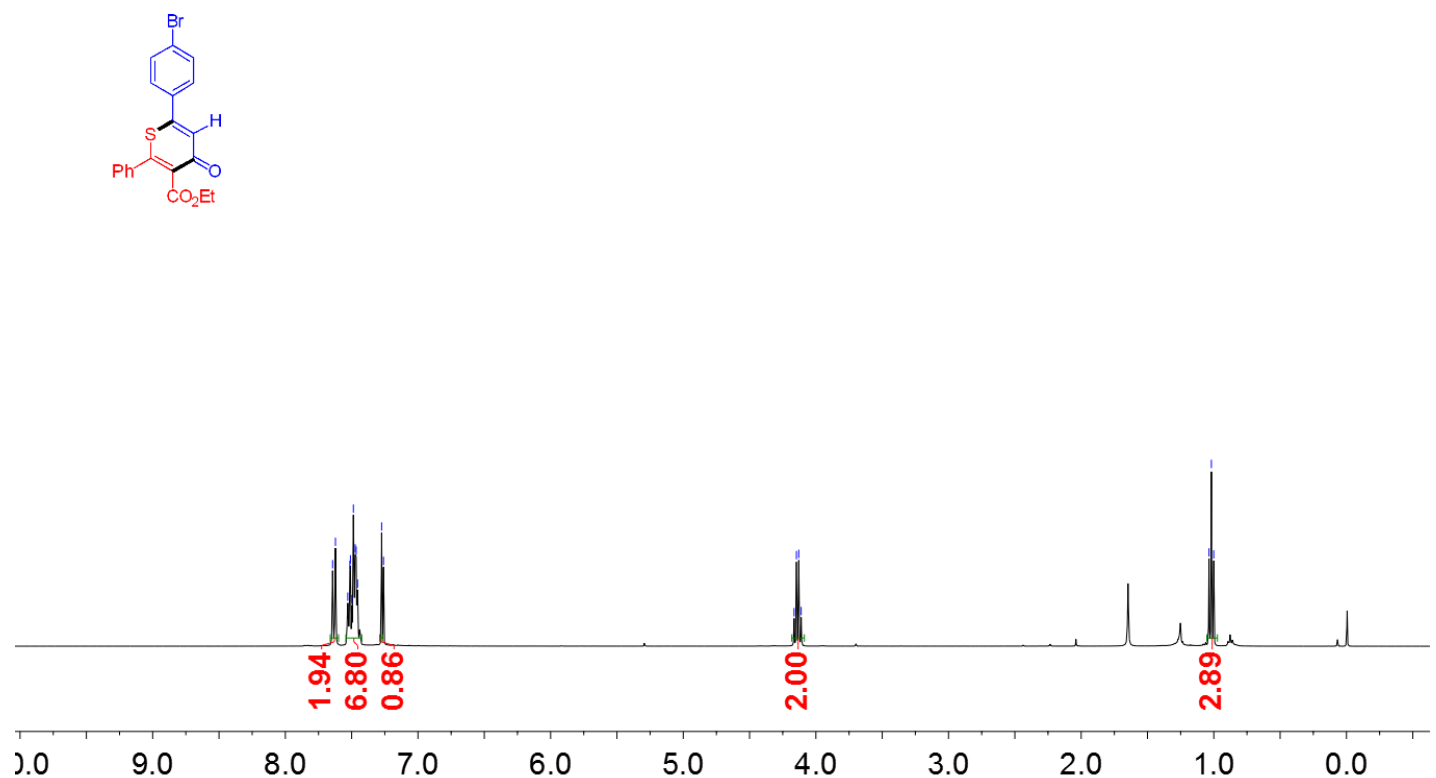

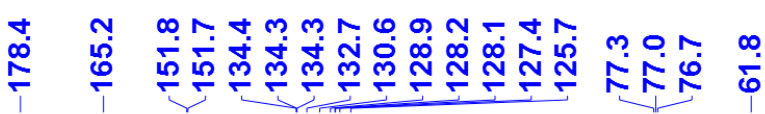

$\stackrel{\sim}{\grave{1}}$

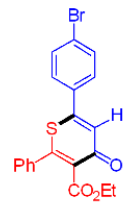

200

180

160

140

120

100

80

60

40

20 
${ }^{1} \mathrm{H}$ and ${ }^{13} \mathrm{C}$ of compound 4 aj in $\mathrm{CDCl}_{3}$

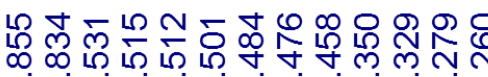

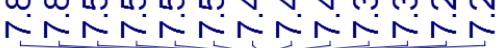

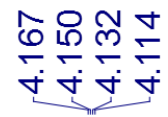

กั ণั
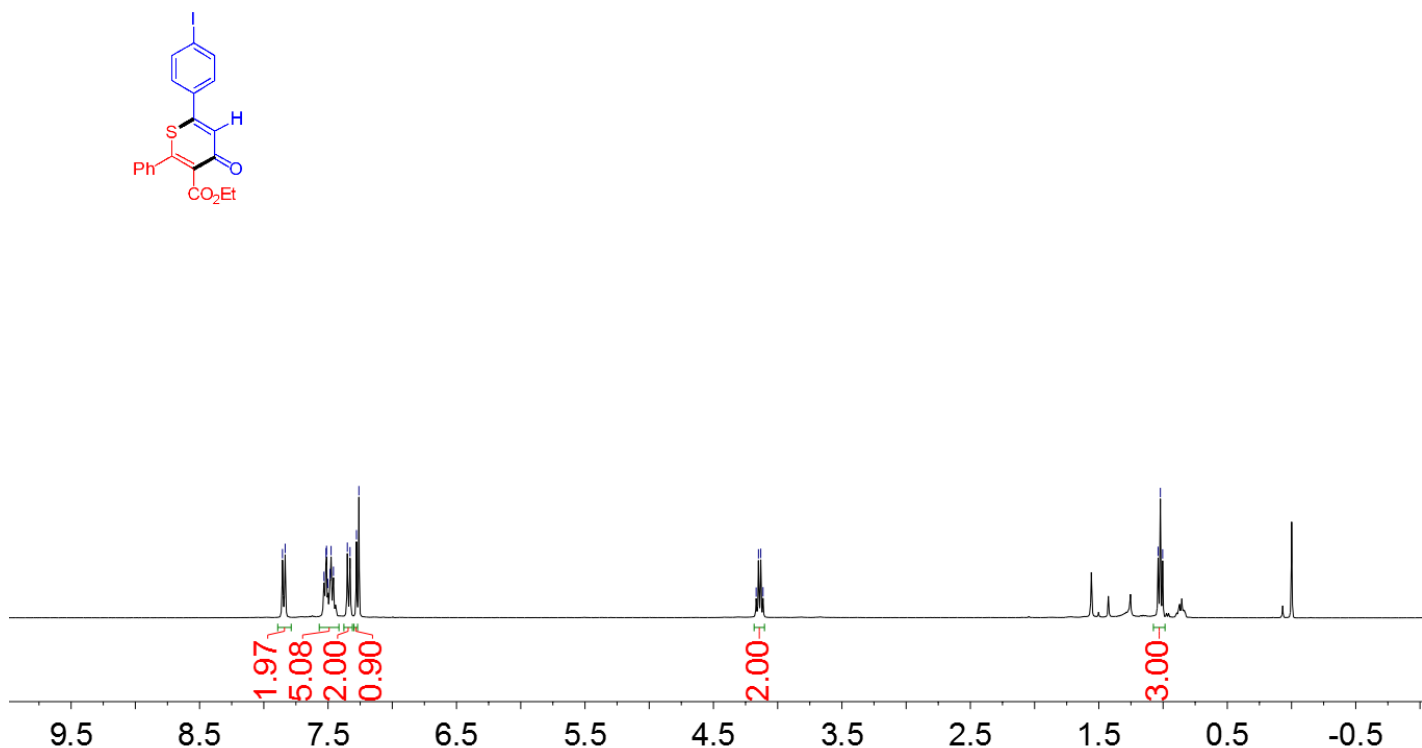

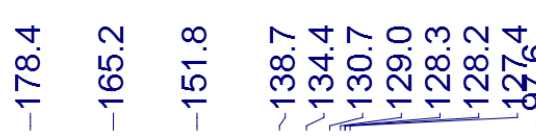

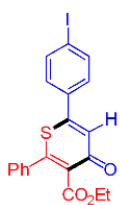

$\bar{\varphi}$

$\stackrel{\infty}{\stackrel{\infty}{\Gamma}}$

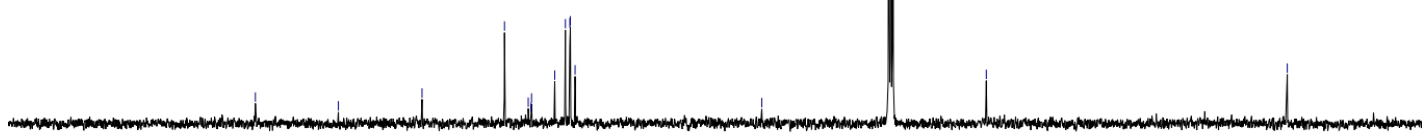

200

180

160

140

120

100

80

60

40

20 
${ }^{1} \mathrm{H}$ and ${ }^{13} \mathrm{C}$ of compound 4 ak in $\mathrm{CDCl}_{3}$

オু হু क NNNNNNNNNNNN

กำ
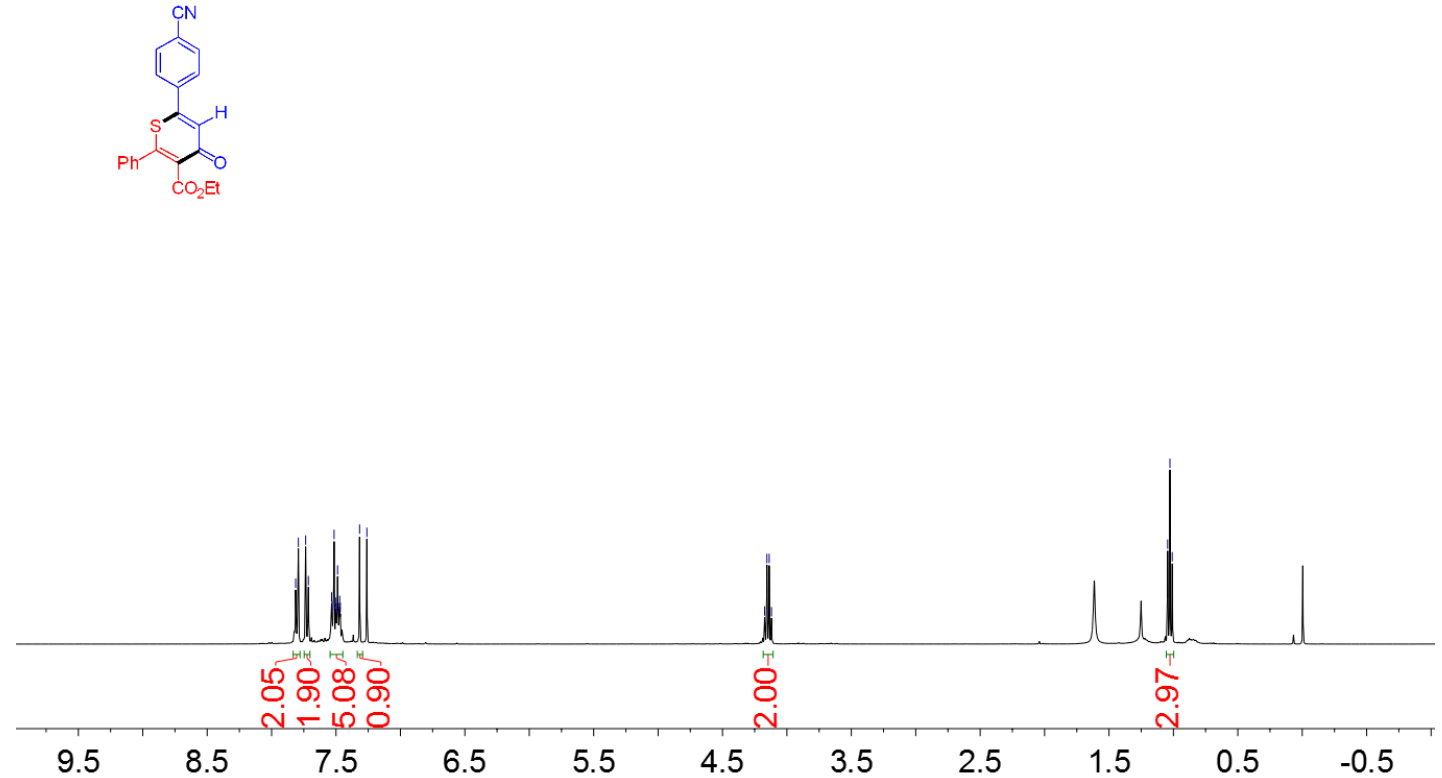

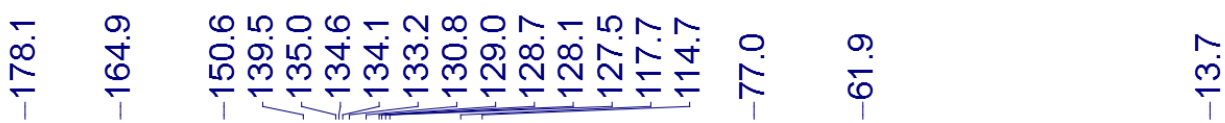
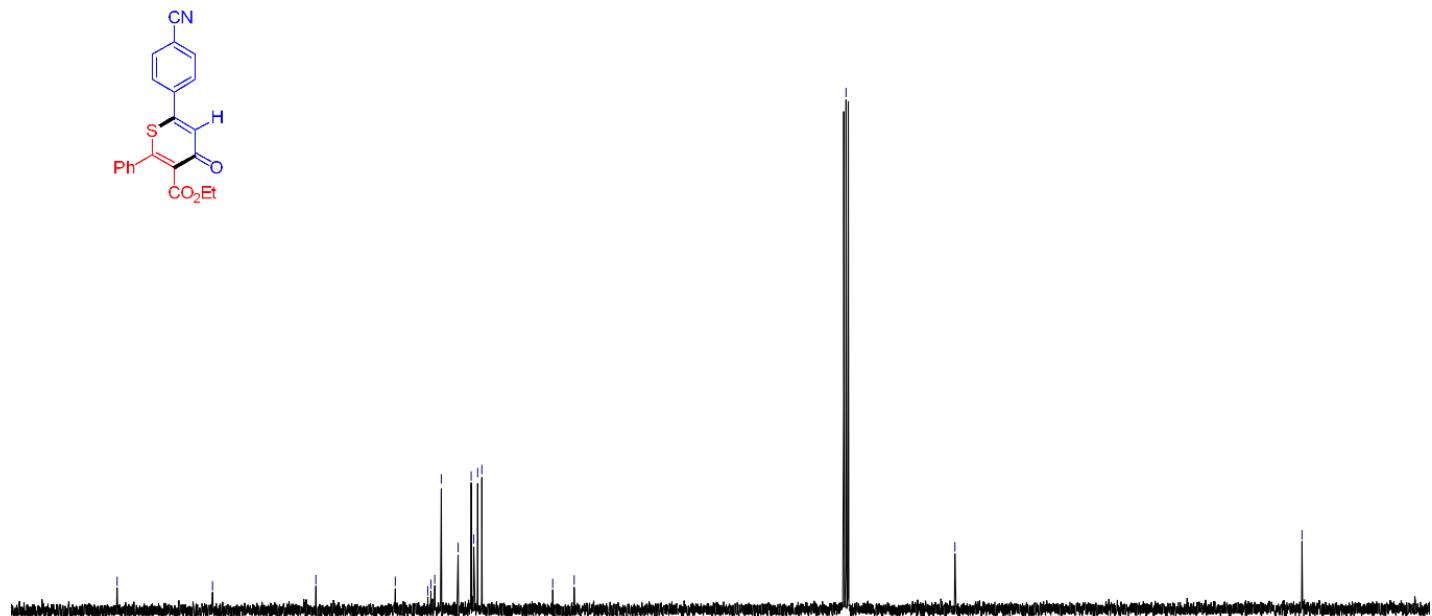
${ }^{1} \mathrm{H}$ and ${ }^{13} \mathrm{C}$ of compound $4 \mathrm{al}$ in $\mathrm{CDCl}_{3}$

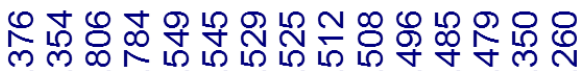

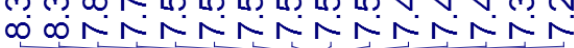

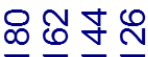

过守守

เุ

$\stackrel{-10}{-}$
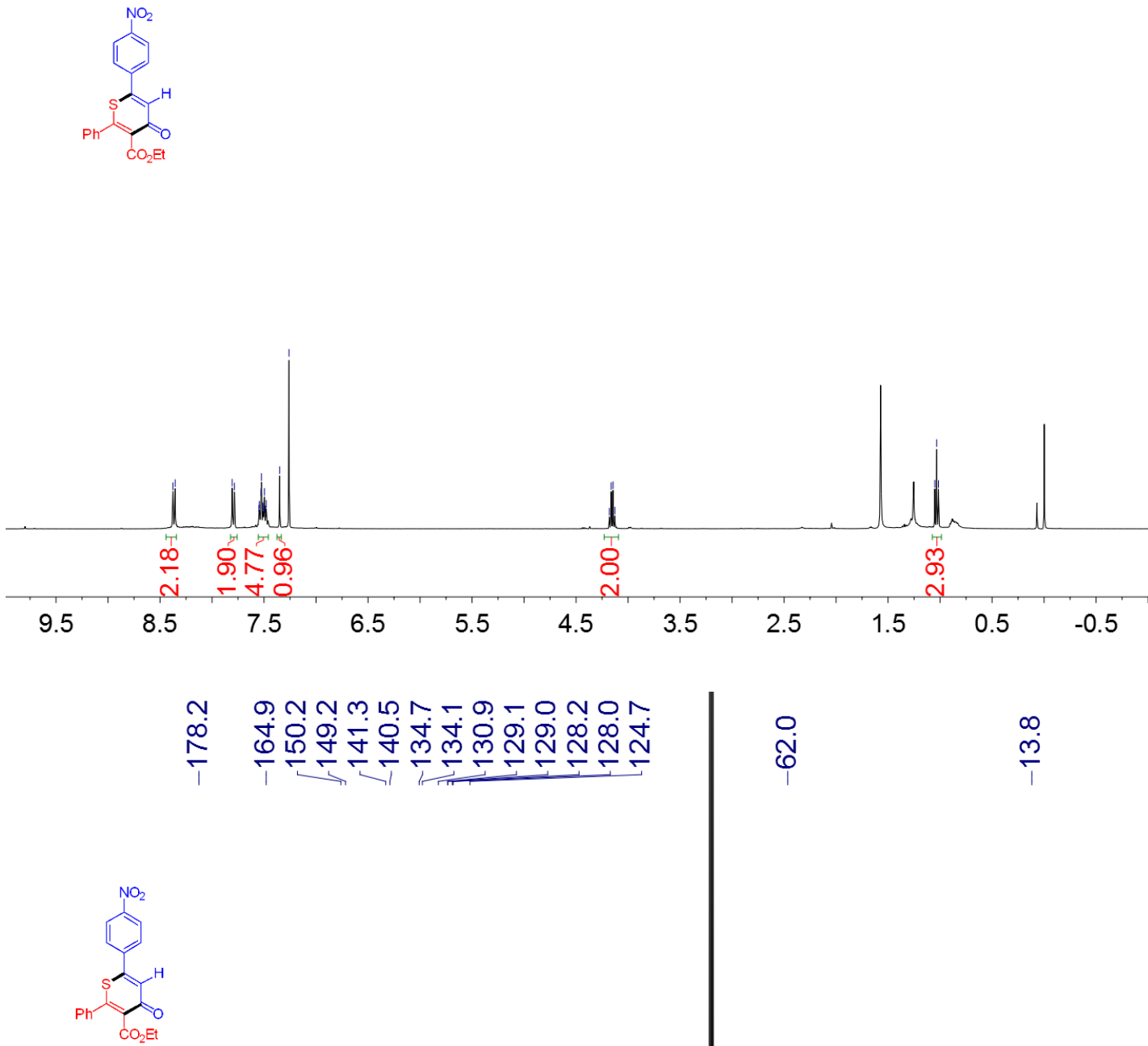

ก

×

ฮิ่

$\stackrel{\infty}{\stackrel{\infty}{\Gamma}}$

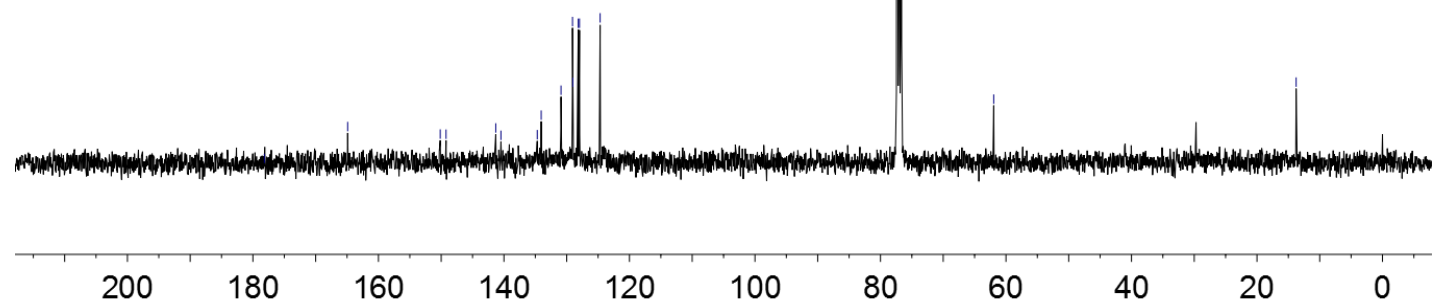


${ }^{1} \mathrm{H}$ and ${ }^{13} \mathrm{C}$ of compound $4 \mathrm{am}$ in $\mathrm{CDCl}_{3}$

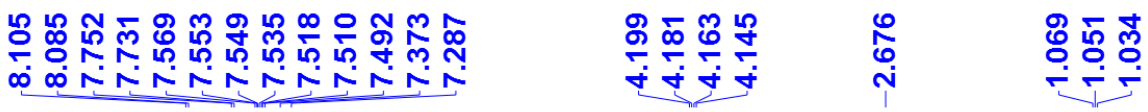

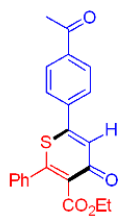

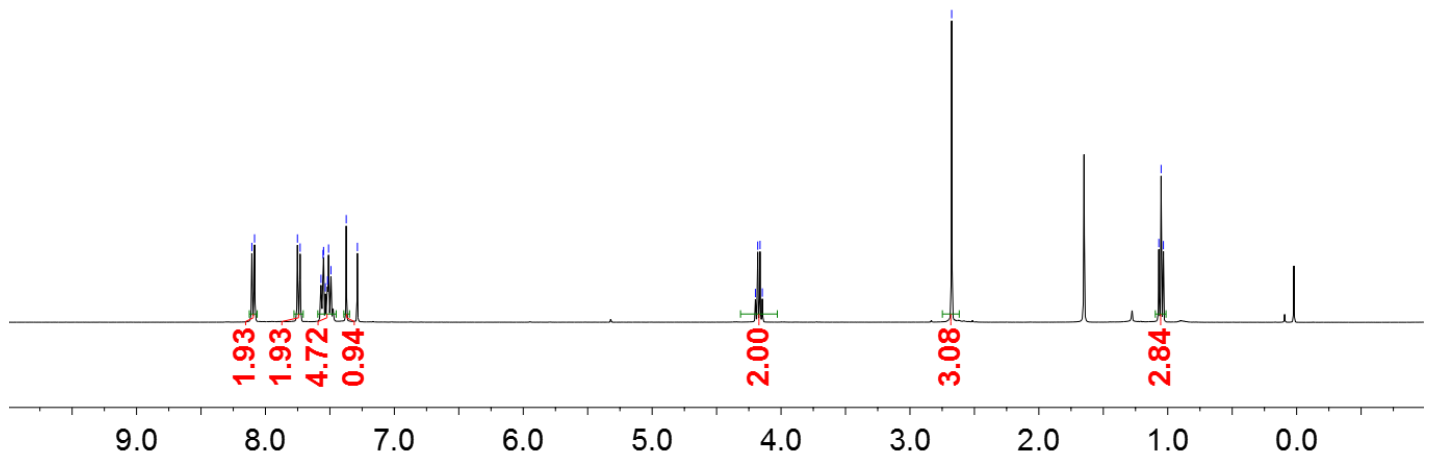

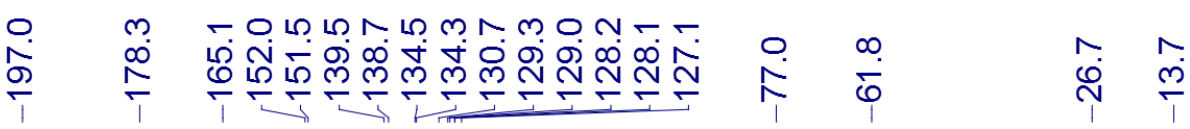
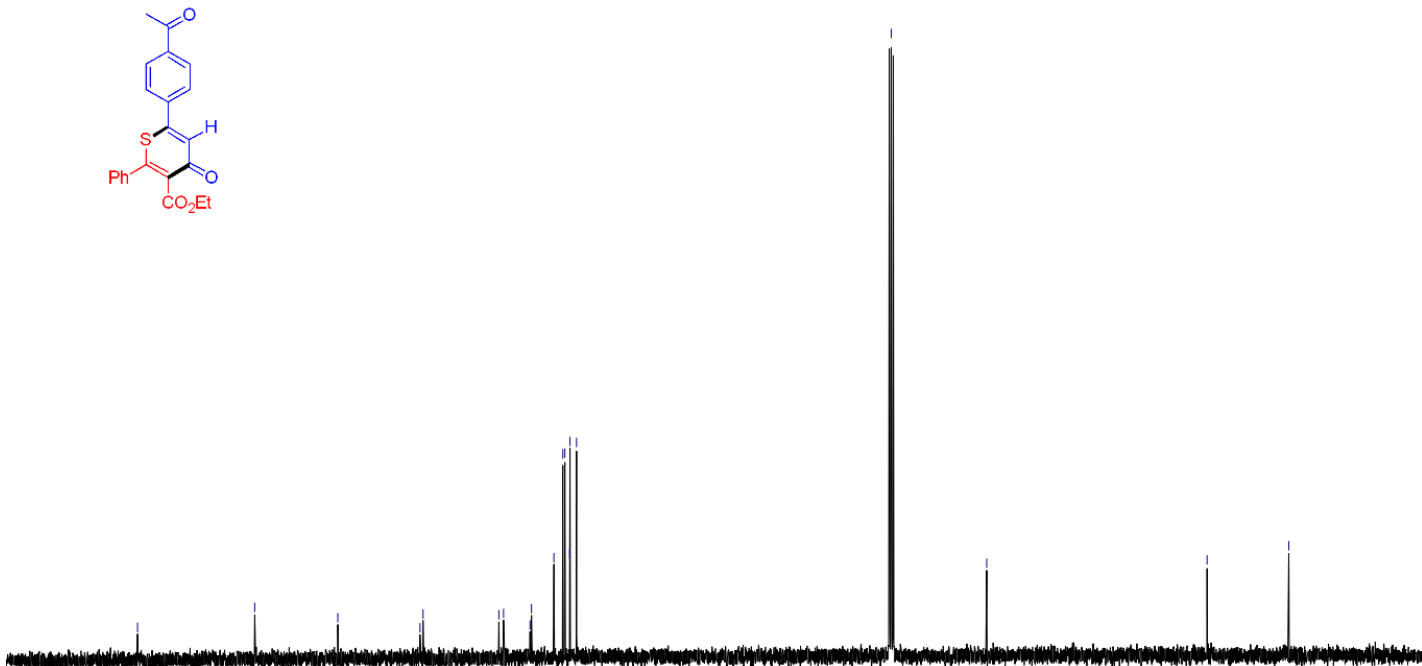
${ }^{1} \mathrm{H}$ and ${ }^{13} \mathrm{C}$ of compound $4 \mathrm{an}$ in $\mathrm{CDCl}_{3}$

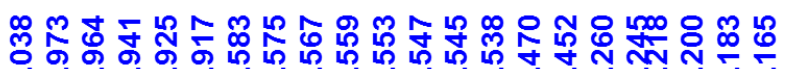

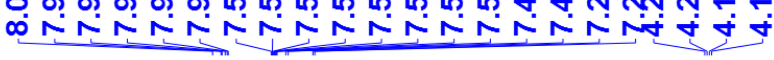

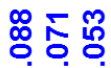
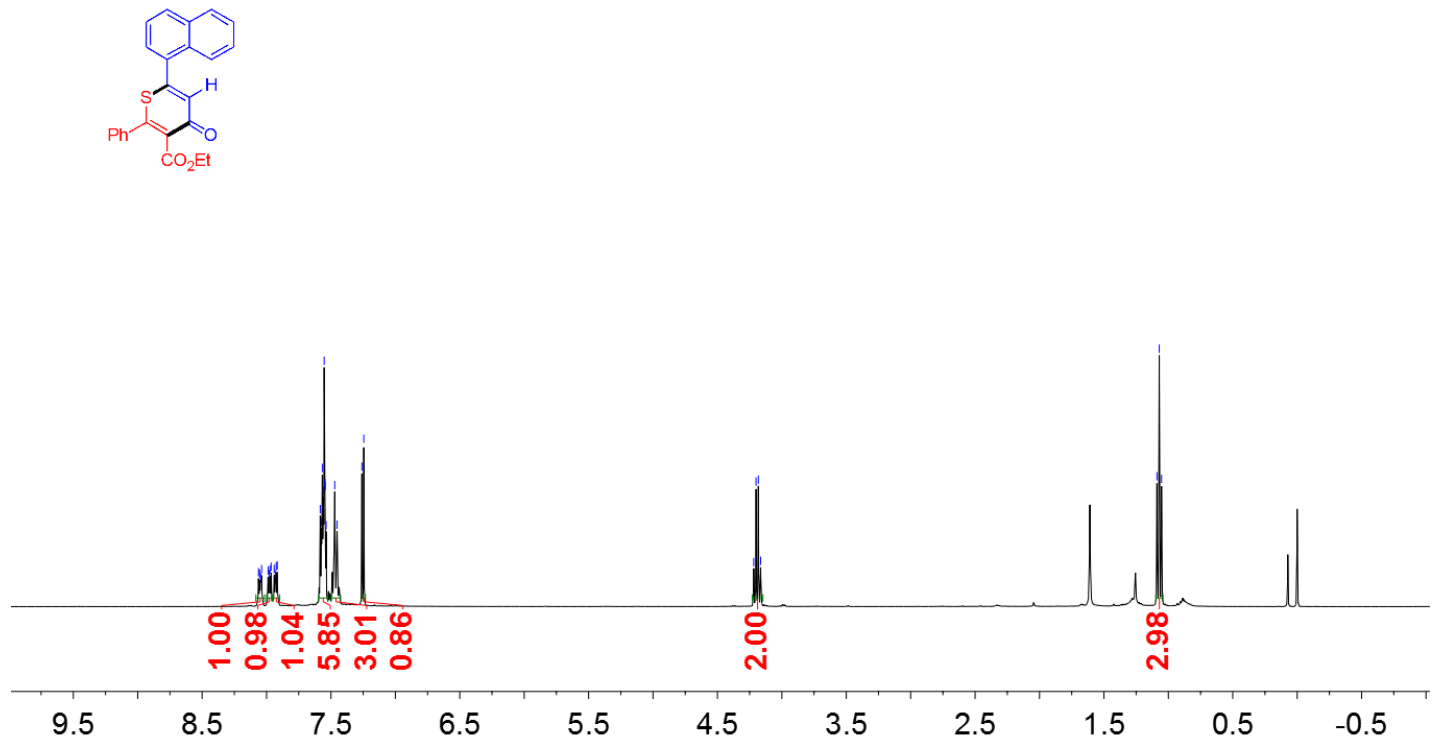

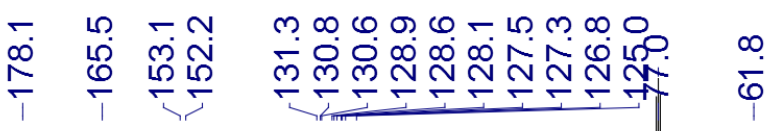

$\stackrel{\infty}{\stackrel{\infty}{\Gamma}}$
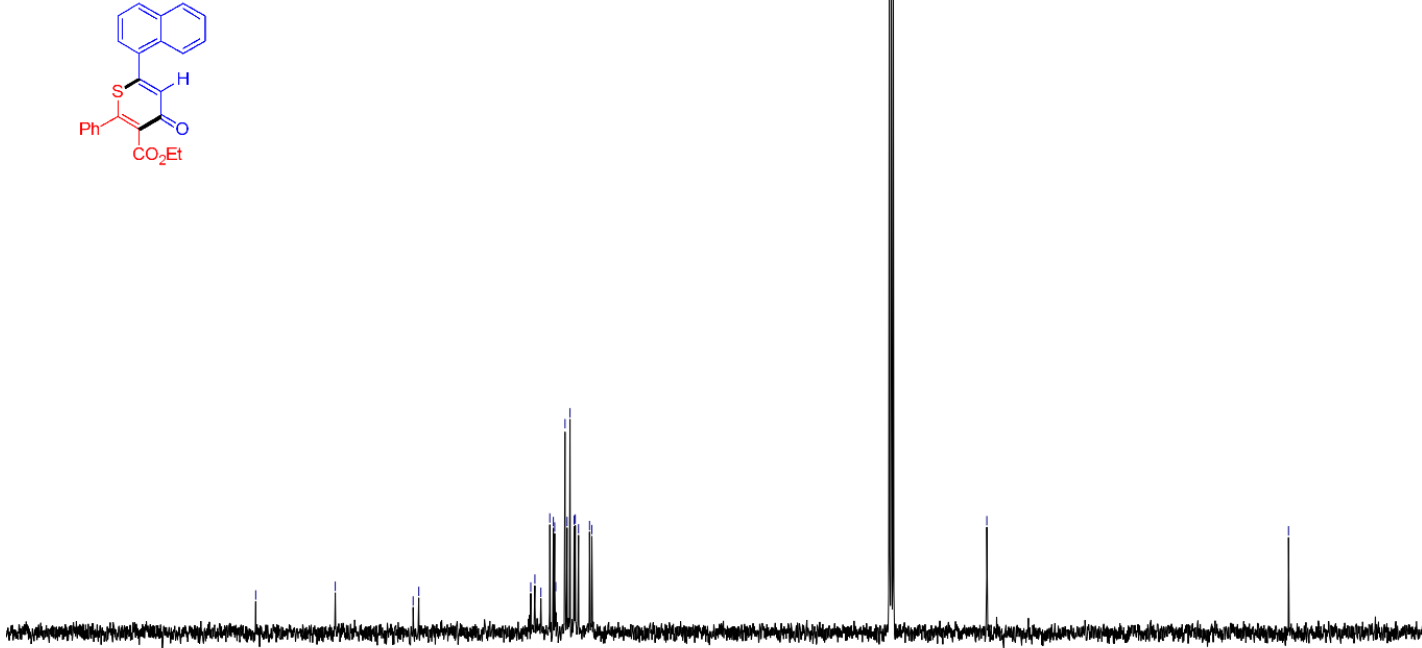

200

180

160

140

120

100

80

60

40

20 
${ }^{1} \mathrm{H}$ and ${ }^{13} \mathrm{C}$ of compound $4 \mathrm{ao}$ in $\mathrm{CDCl}_{3}$

ஸ ำ 00 का

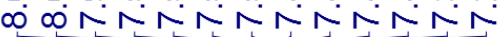
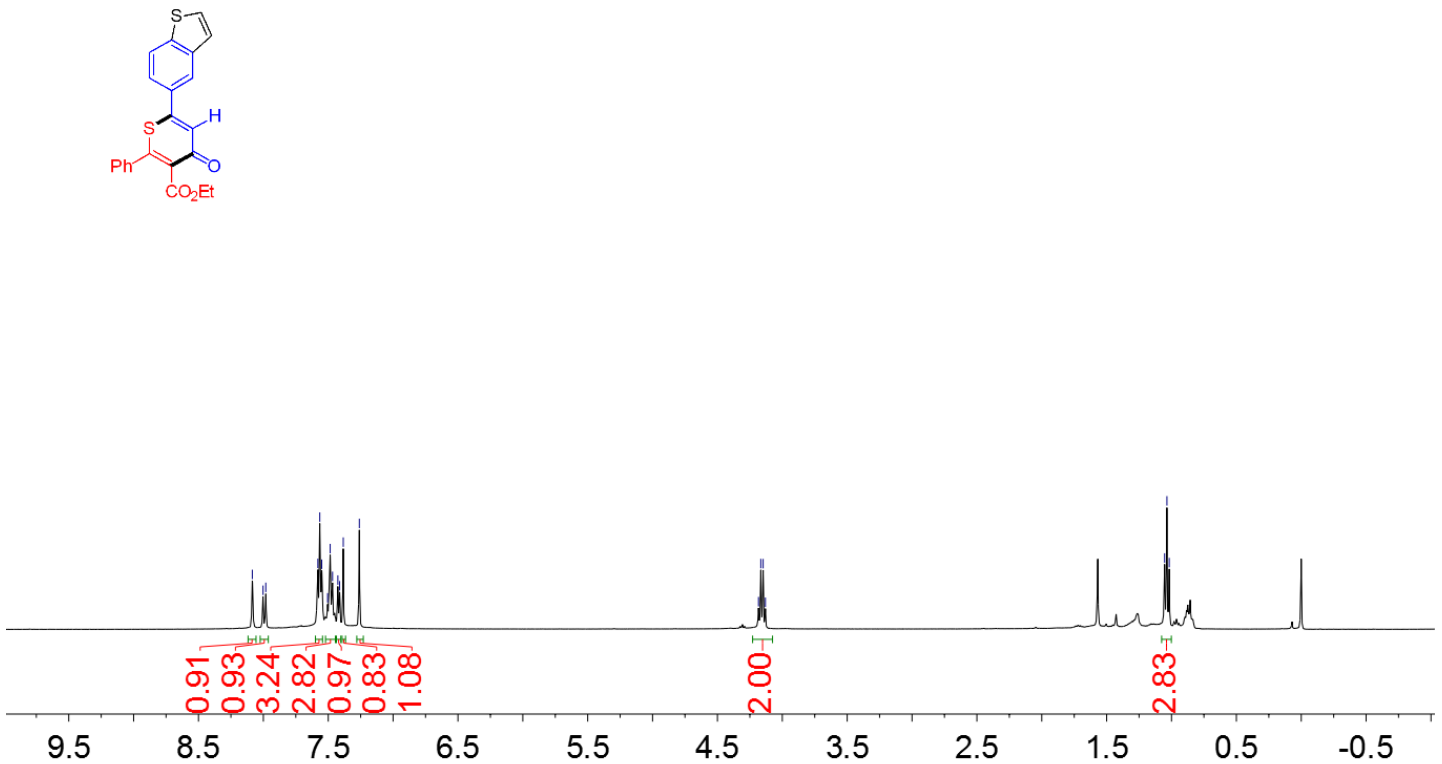

ம. $\quad$

․
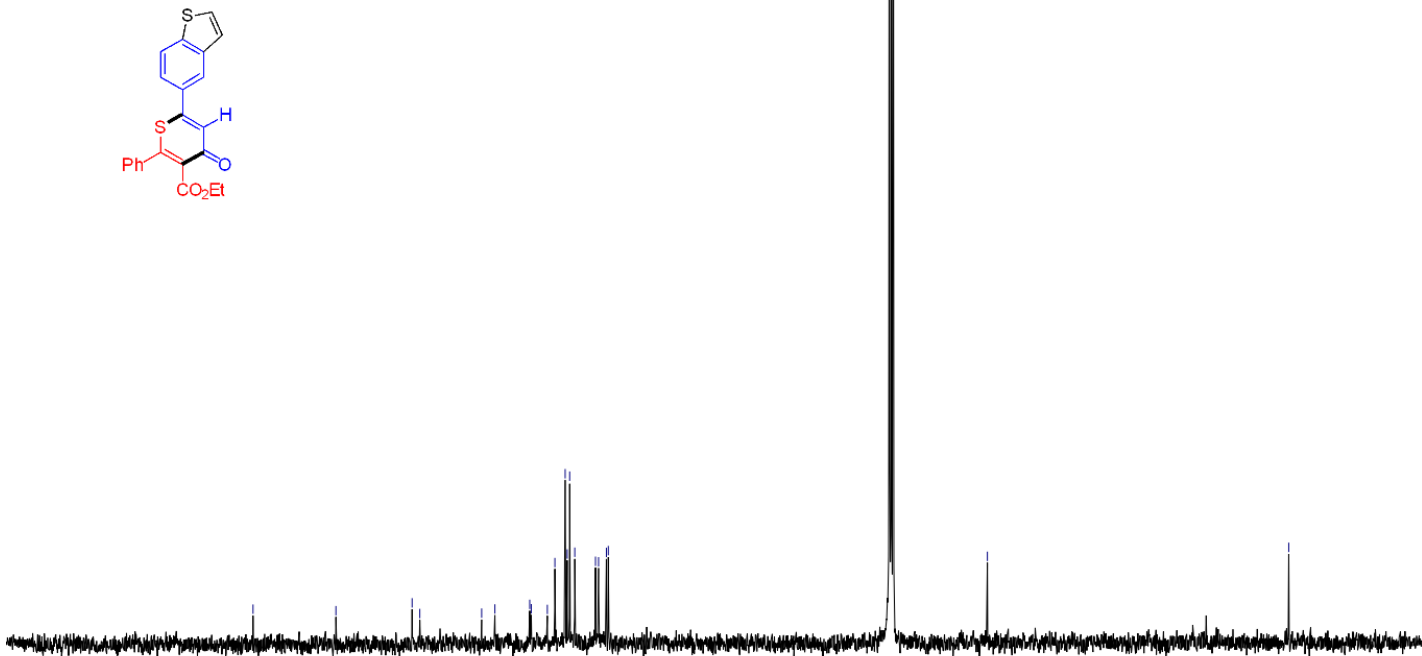
${ }^{1} \mathrm{H}$ and ${ }^{13} \mathrm{C}$ of compound 4ap in $\mathrm{CDCl}_{3}$

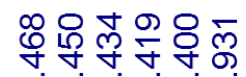
NNNNG

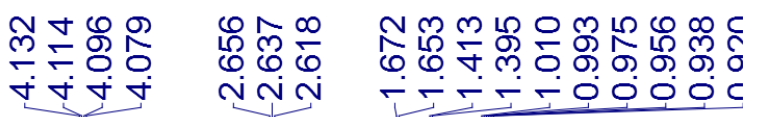
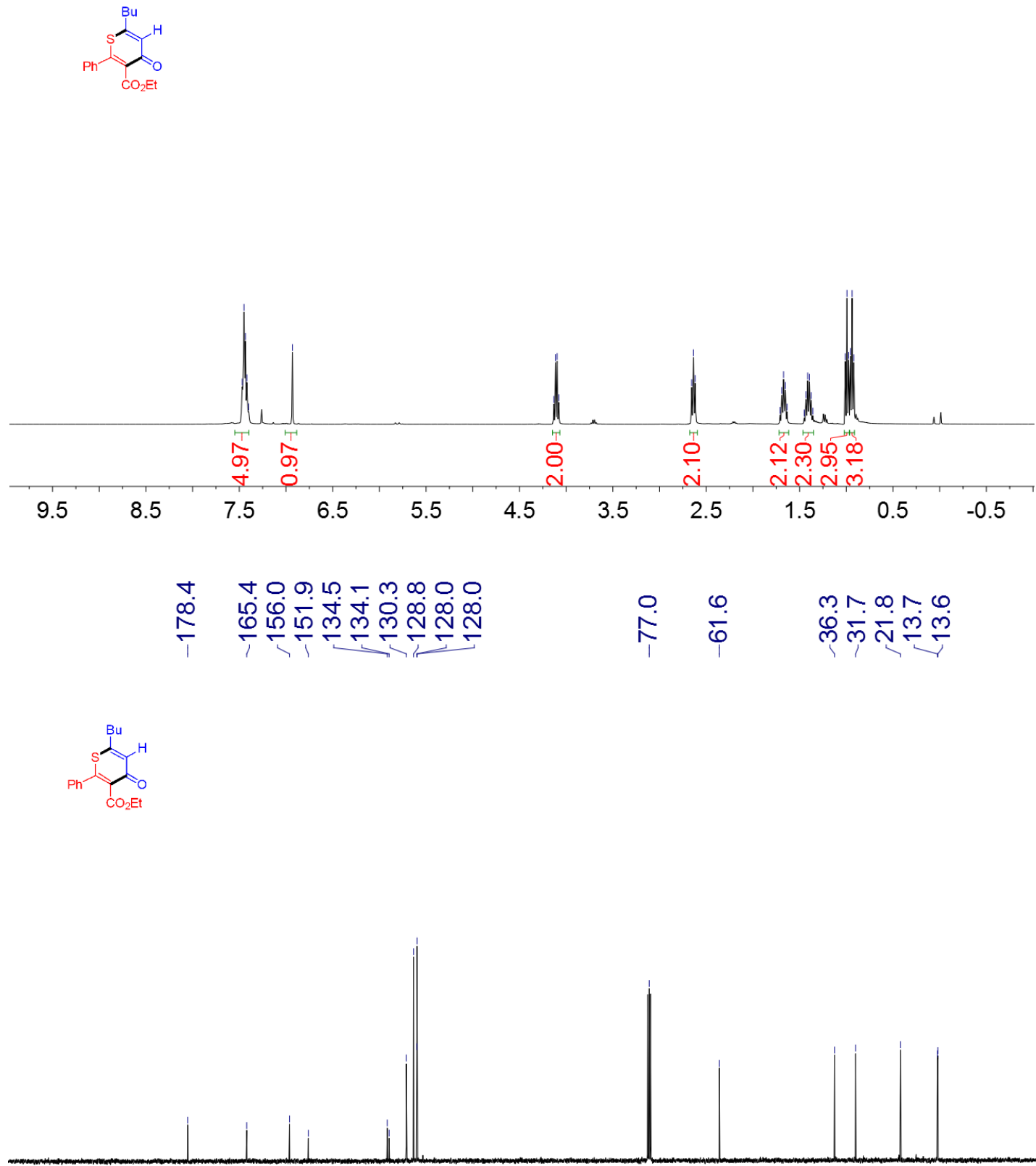

$140 \quad 120$

80

60

40 
${ }^{1} \mathrm{H}$ and ${ }^{13} \mathrm{C}$ of compound $4 \mathrm{aq}$ in $\mathrm{CDCl}_{3}$

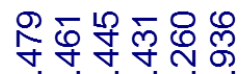

NNNNG

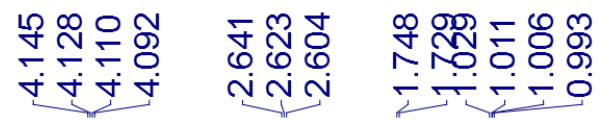
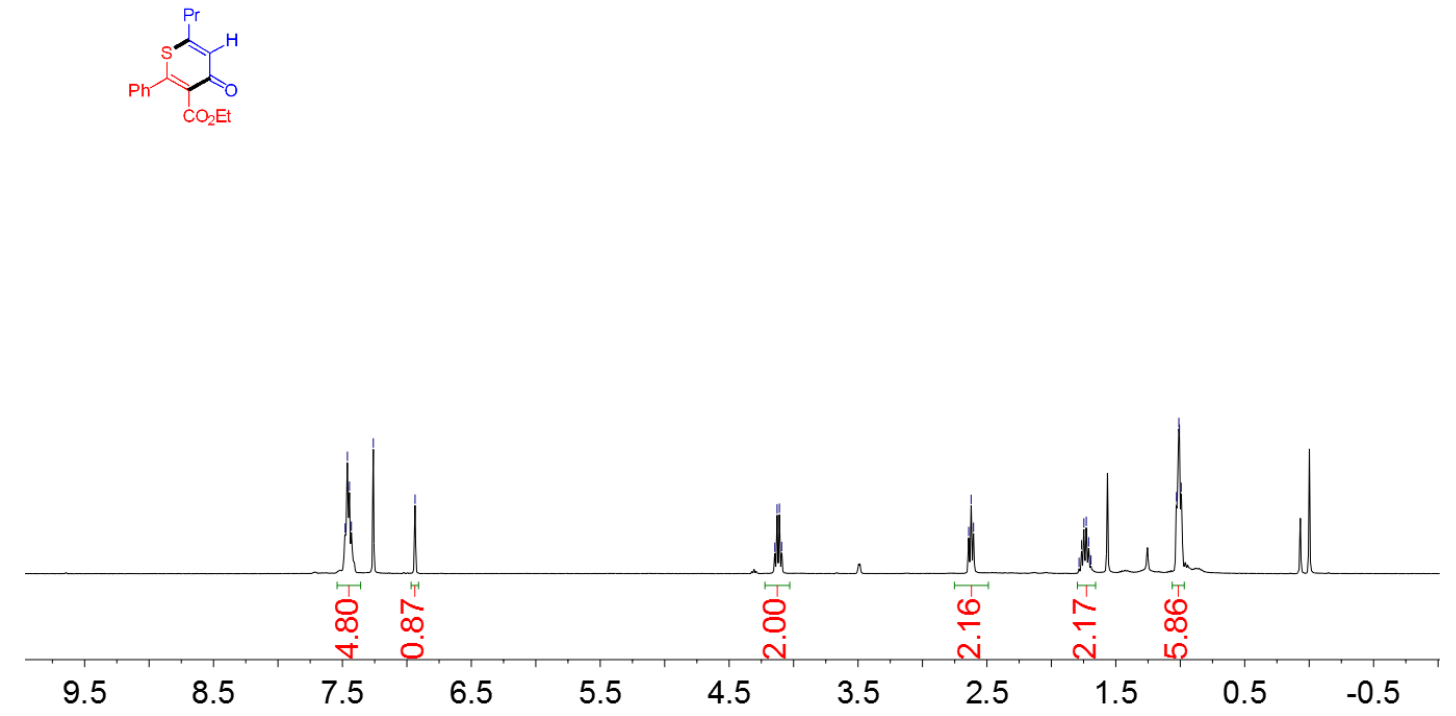

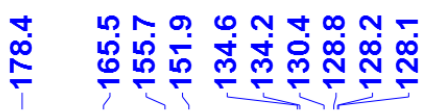

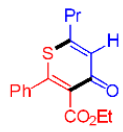

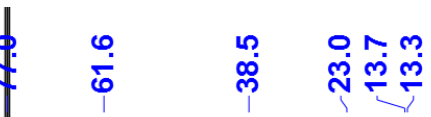

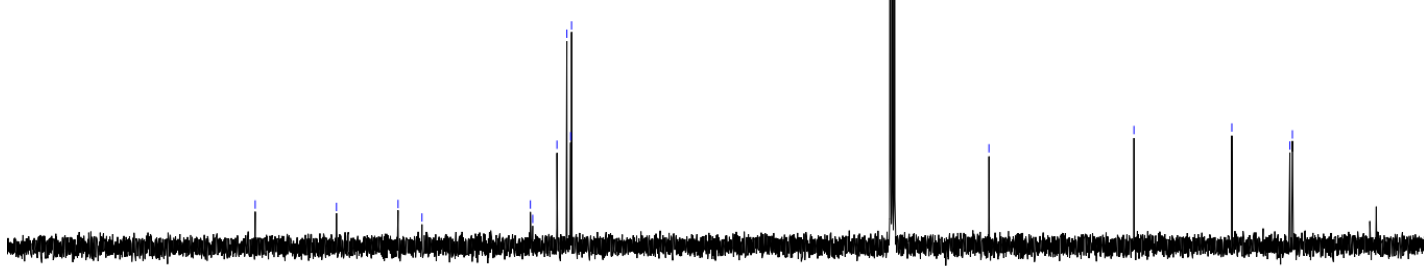


${ }^{1} \mathrm{H}$ and ${ }^{13} \mathrm{C}$ of compound $4 \mathrm{ar}$ in $\mathrm{CDCl}_{3}$

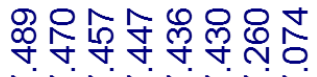
NNNNNNN

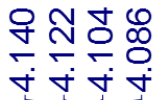

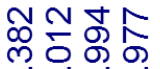

-

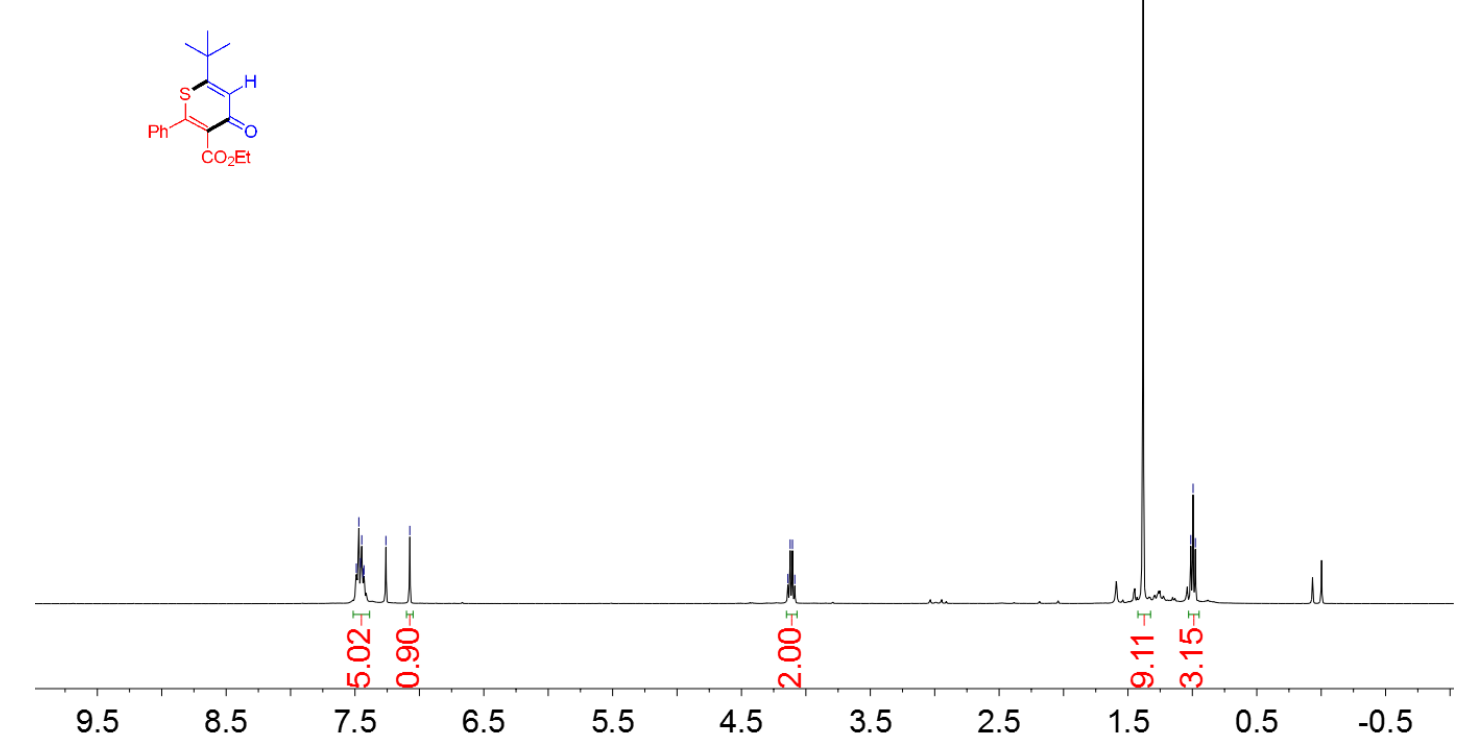

is

5

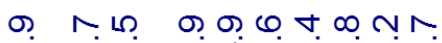

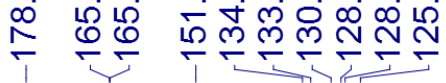

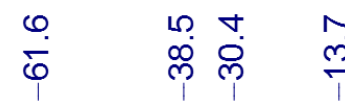

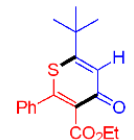

200

180

160

140

120

100

80

60

40

20 
${ }^{1} \mathrm{H}$ and ${ }^{13} \mathrm{C}$ of compound 4 as in $\mathrm{CDCl}_{3}$

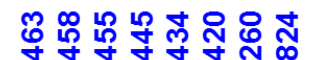

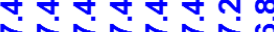

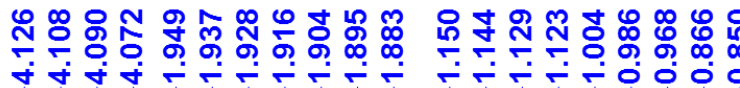

$\underbrace{\mathrm{H}}_{\mathrm{CO}_{2} \mathrm{Et}}$

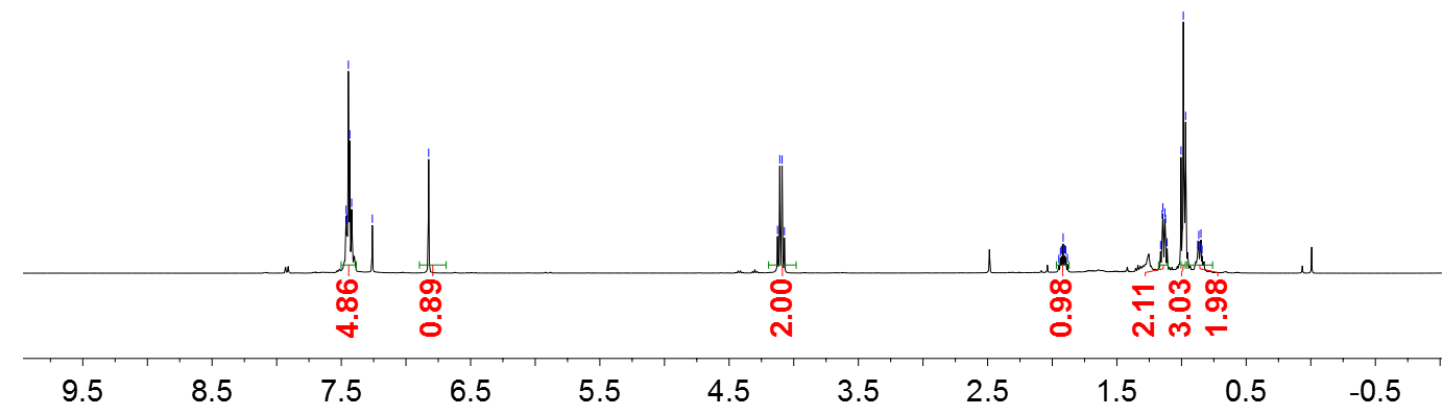

ก

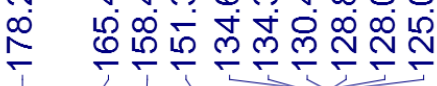

mot 6

NヘN চே

ติ

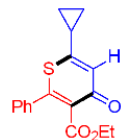

200

180

160

140

120

100

80

60

40 
${ }^{1} \mathrm{H}$ and ${ }^{13} \mathrm{C}$ of compound 4 at in $\mathrm{CDCl}_{3}$

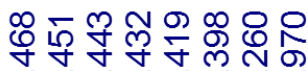

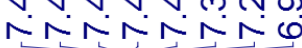

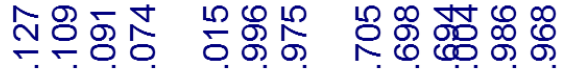

+i
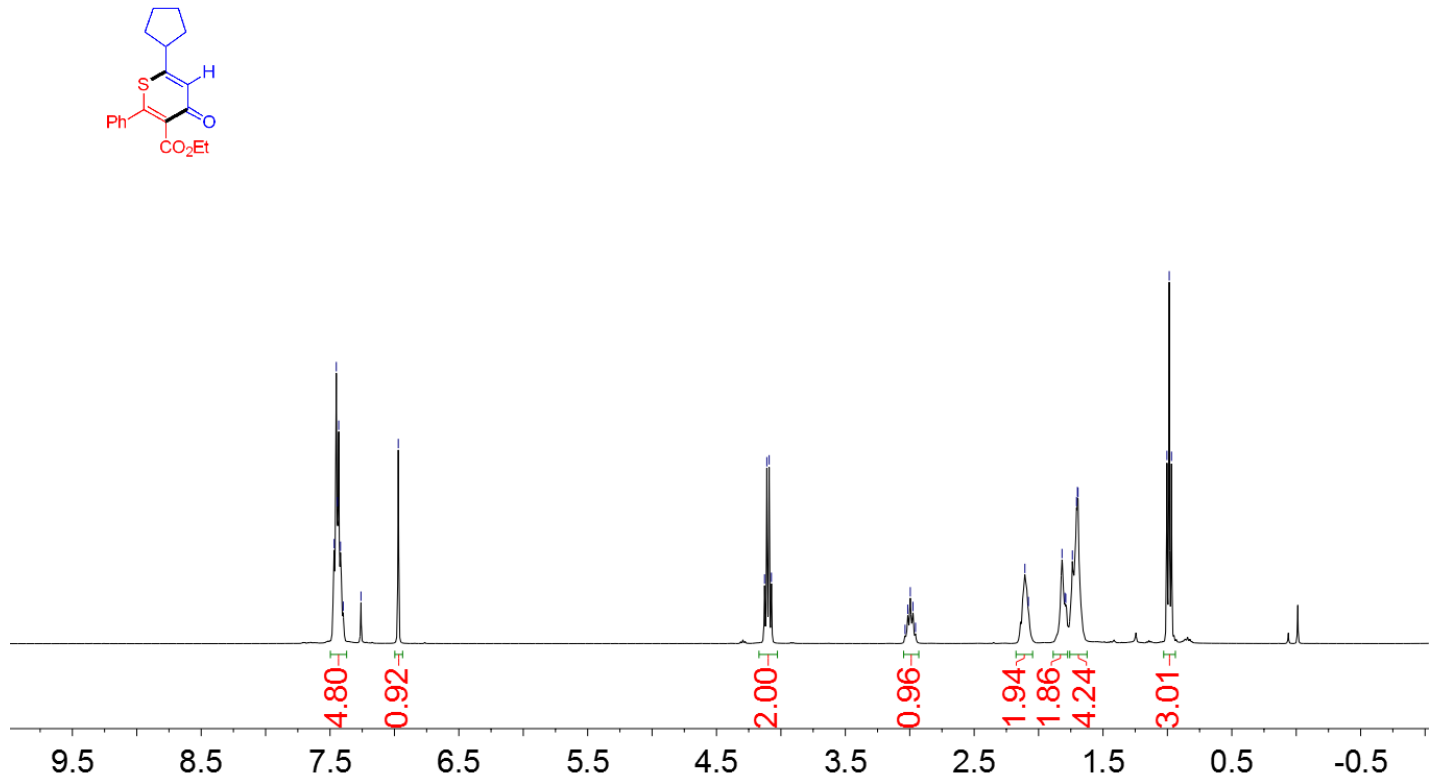

L.

事 $\quad$\begin{tabular}{l}
0 \\
\hline
\end{tabular}
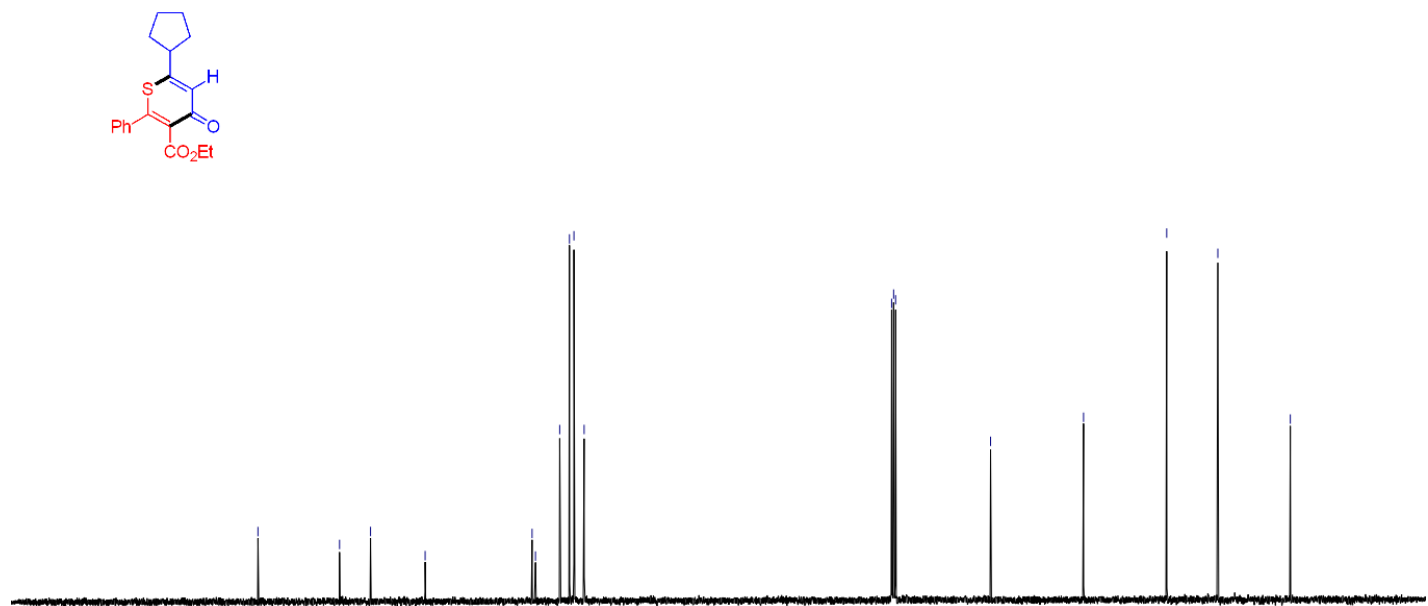
${ }^{1} \mathrm{H}$ and ${ }^{13} \mathrm{C}$ of compound $4 \mathrm{au}$ in $\mathrm{CDCl}_{3}$

守

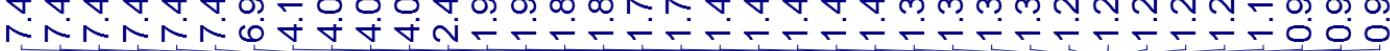
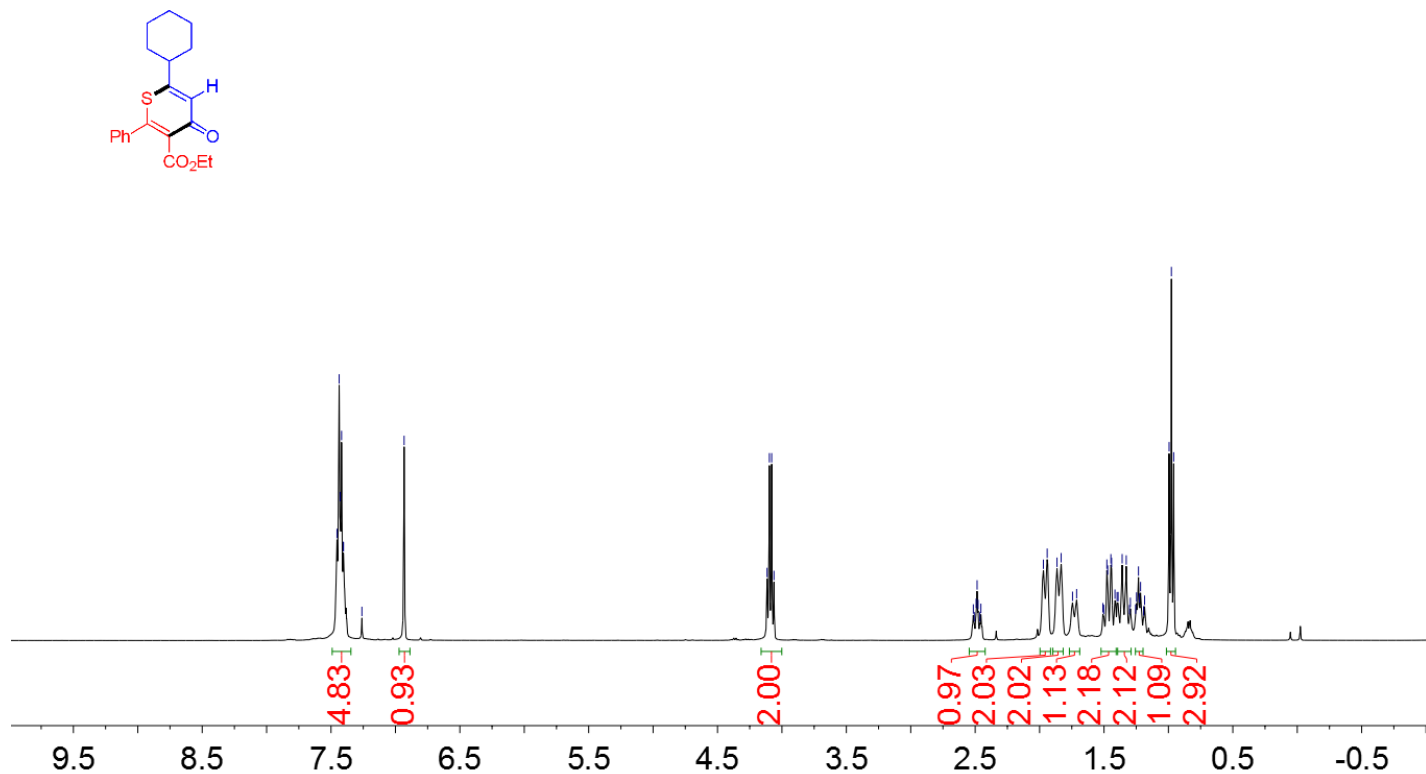

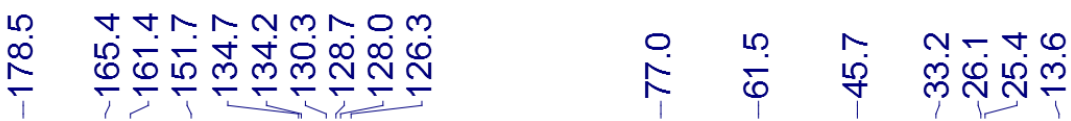
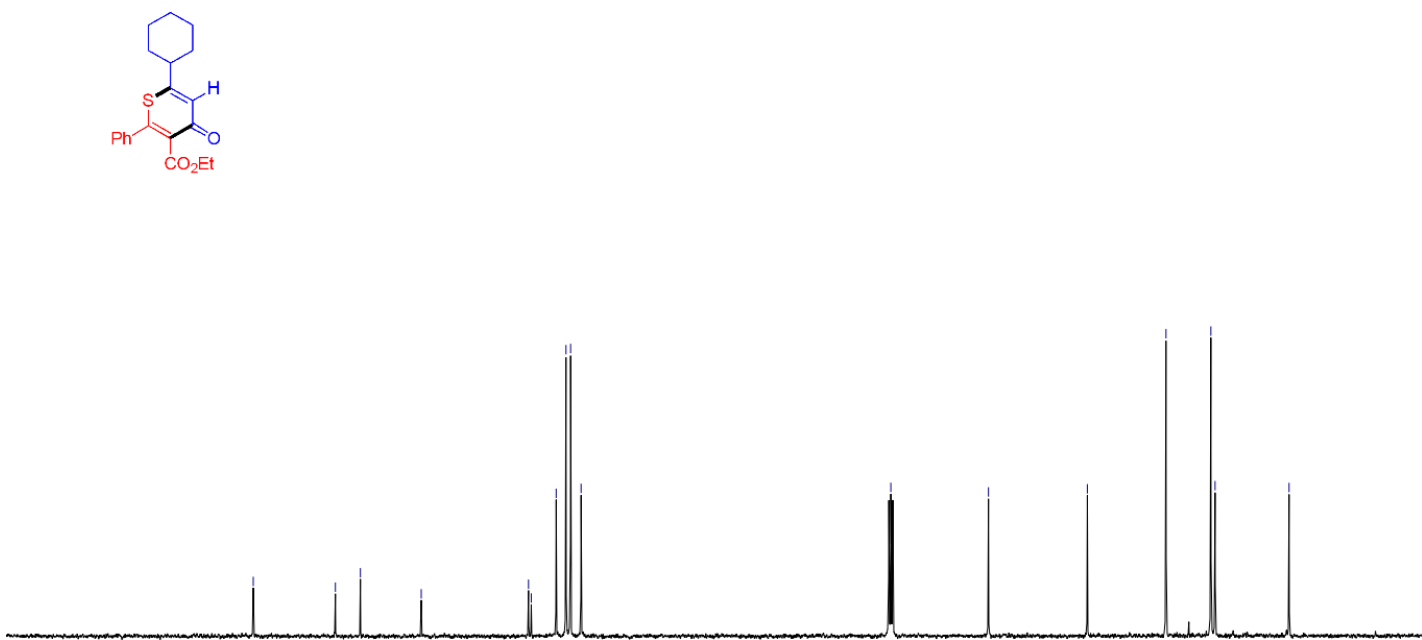

200

160

140

120

100

80

60

40

20 
${ }^{1} \mathrm{H}$ and ${ }^{13} \mathrm{C}$ of compound $6 \mathrm{aa}$ in $\mathrm{CDCl}_{3}$

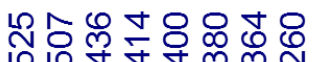
NNNNNN

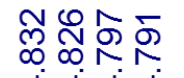

ํํำ

जं लं लं लं लं
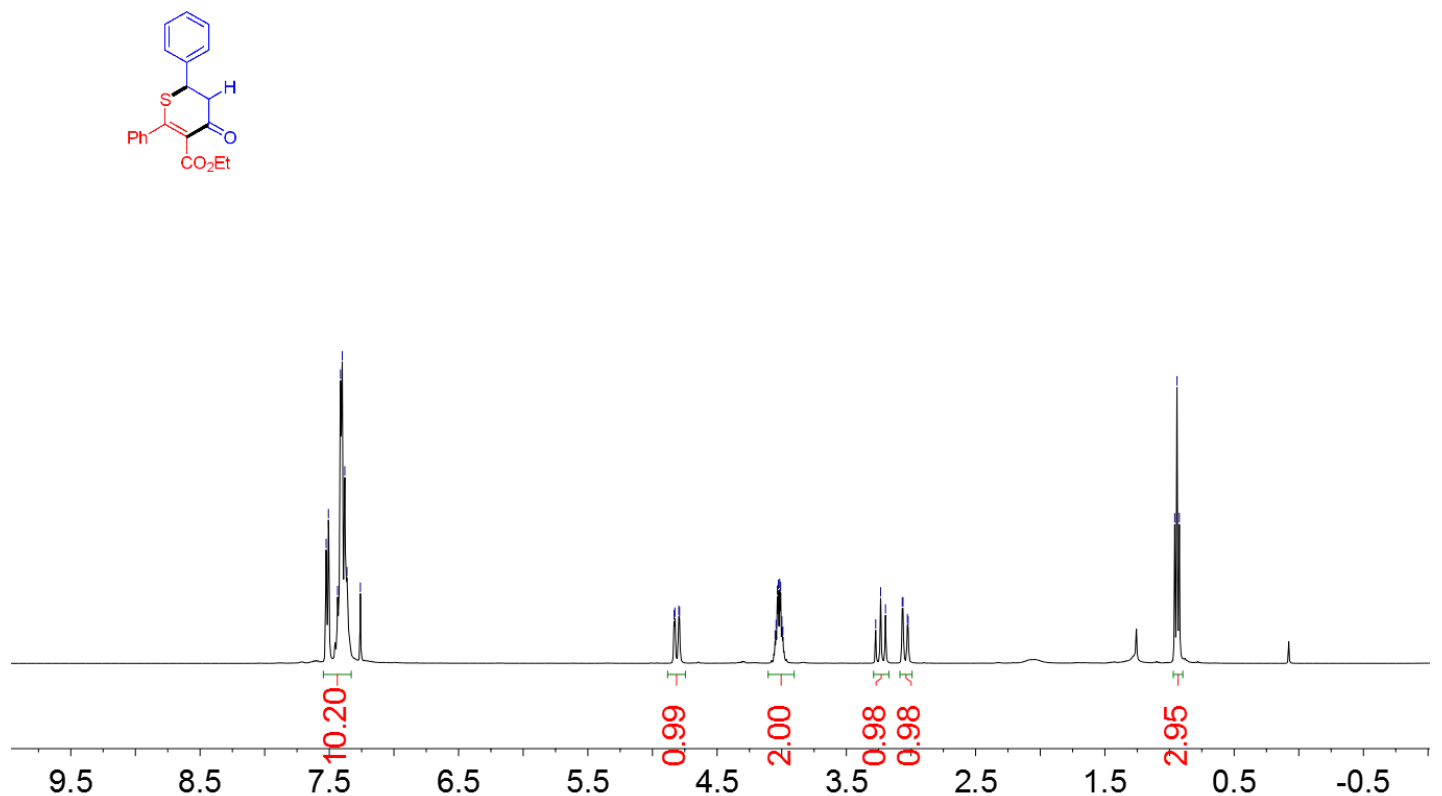

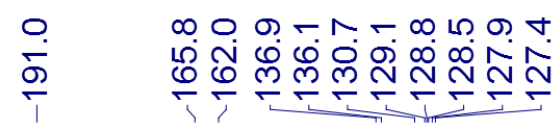

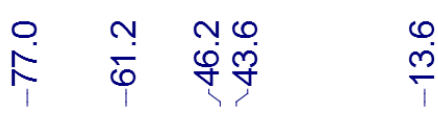

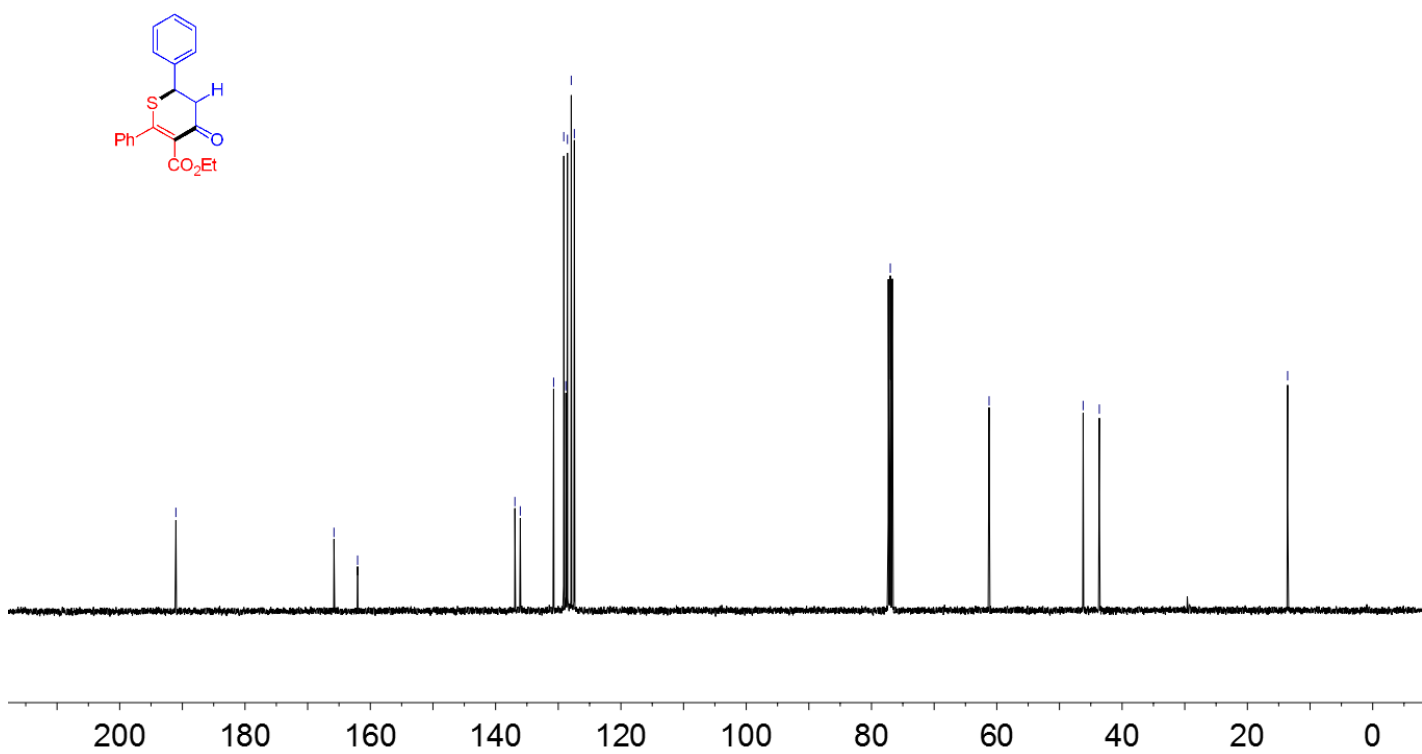


${ }^{1} \mathrm{H}$ and ${ }^{13} \mathrm{C}$ of compound $6 \mathrm{ab}$ in $\mathrm{CDCl}_{3}$

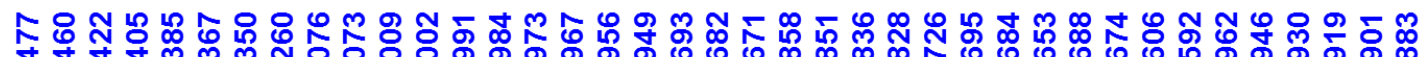

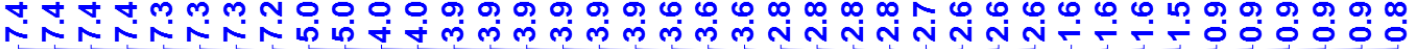
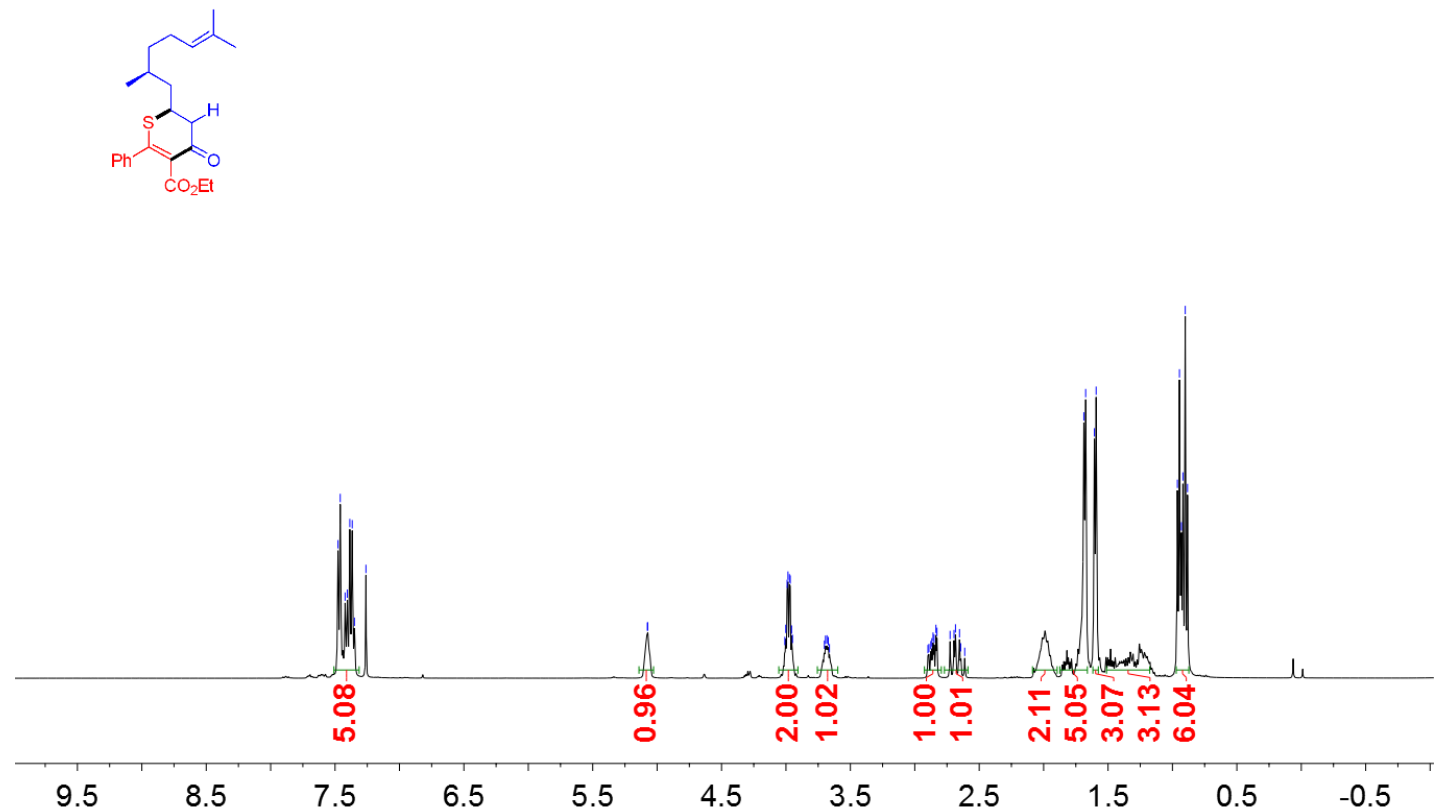

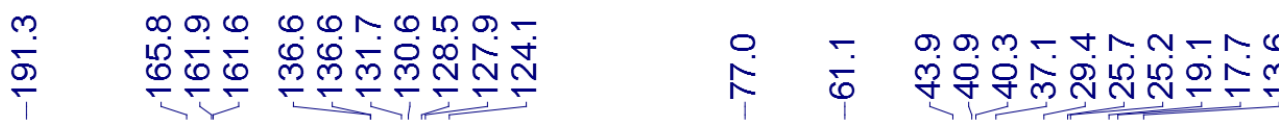

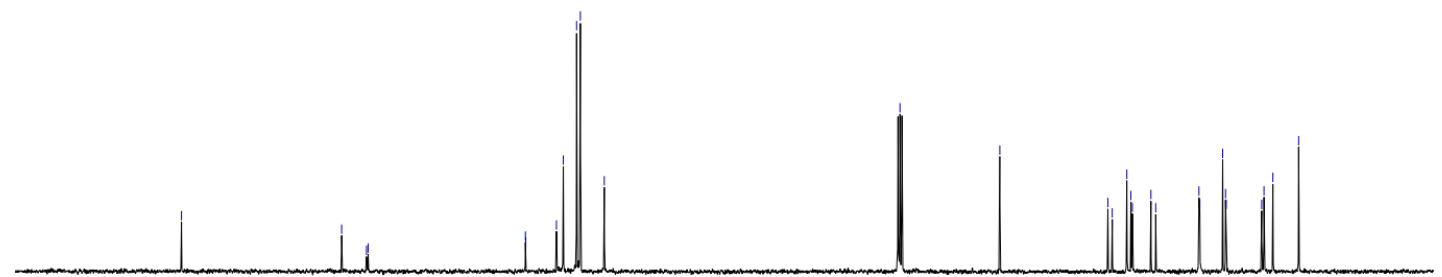

200 180 160 140 120 100 80 60 20 
${ }^{1} \mathrm{H}$ and ${ }^{13} \mathrm{C}$ of compound $6 \mathrm{ac}$ in $\mathrm{CDCl}_{3}$

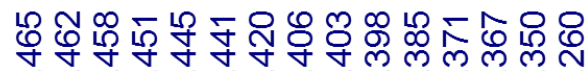
NNNNNNNNNNNN

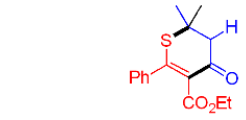

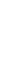

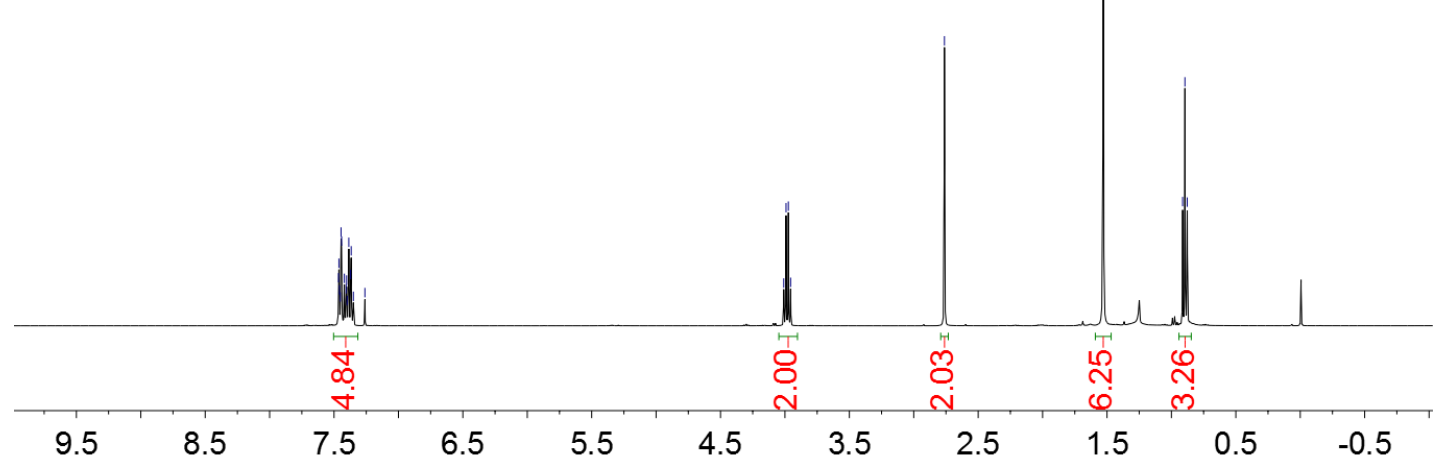

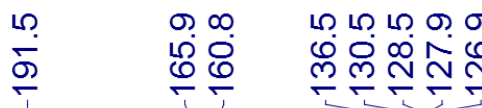

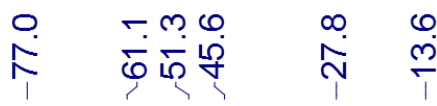
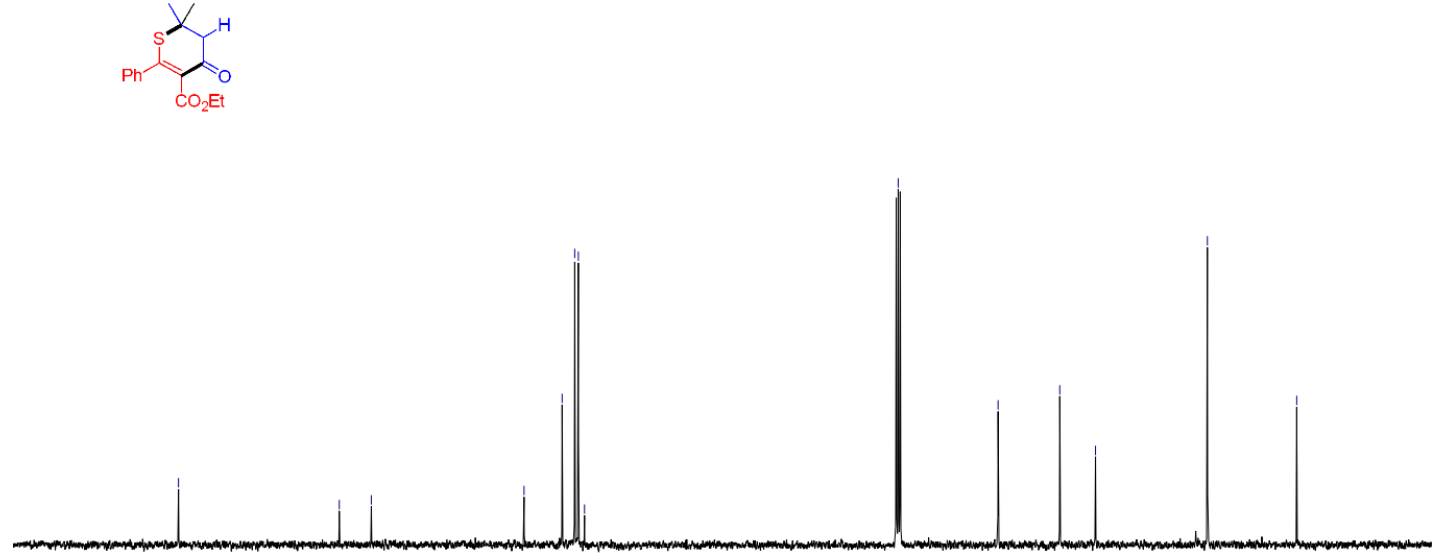

200 180 160

140

120

100

80

60

40

20 
${ }^{1} \mathrm{H}$ and ${ }^{13} \mathrm{C}$ of compound $6 \mathrm{ad}$ in $\mathrm{CDCl}_{3}$
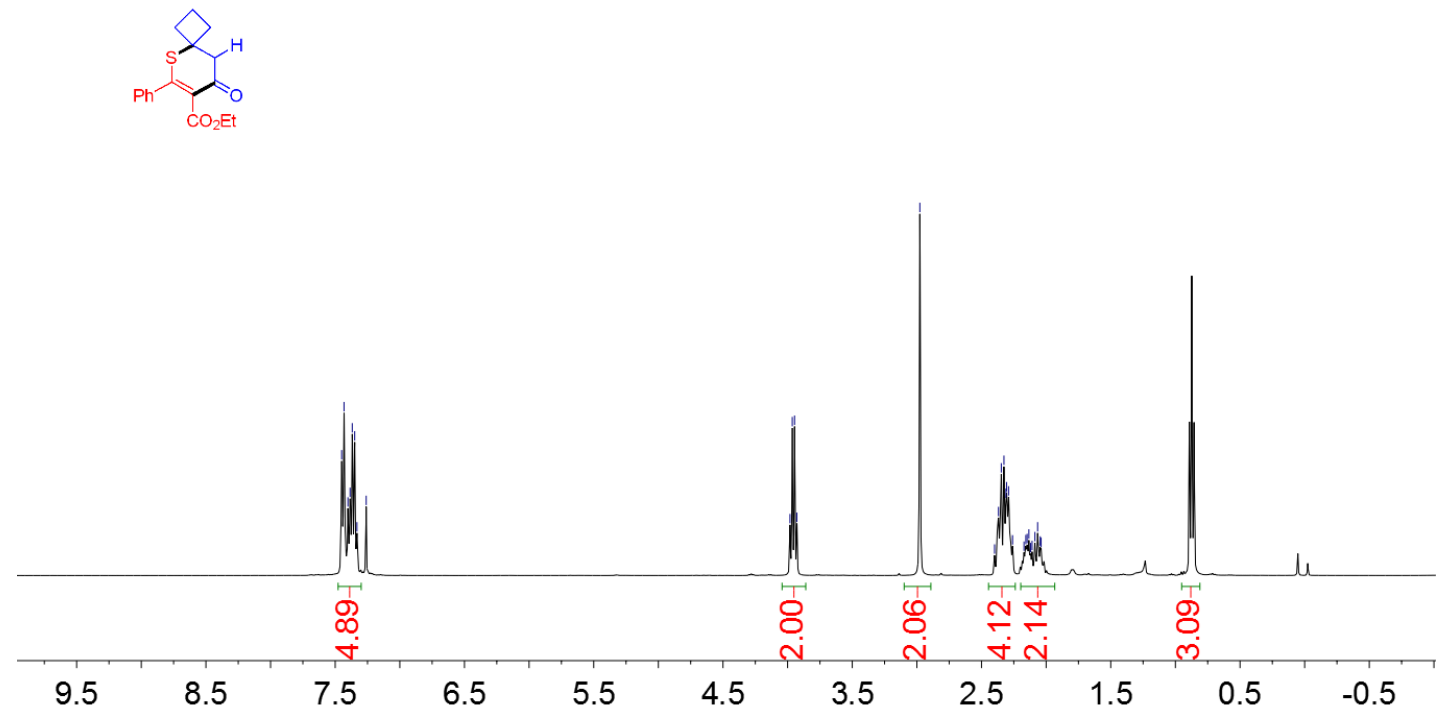

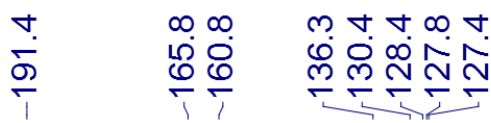

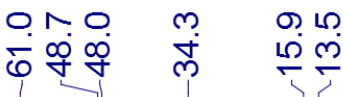

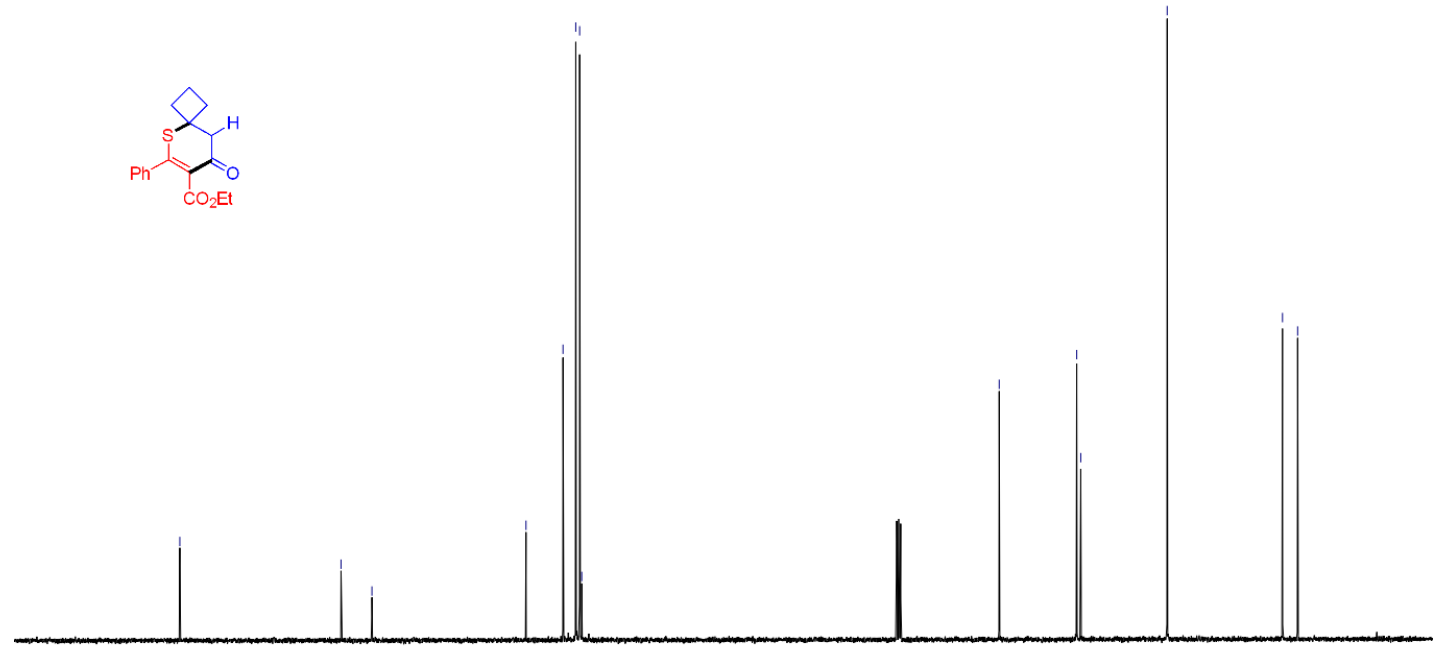

200

180

160

140

120

100

80

60

40

20 
${ }^{1} \mathrm{H}$ and ${ }^{13} \mathrm{C}$ of compound 6ae in $\mathrm{CDCl}_{3}$

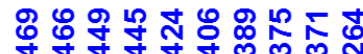

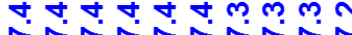

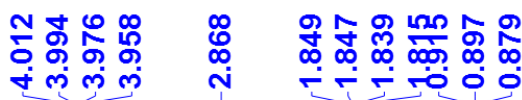
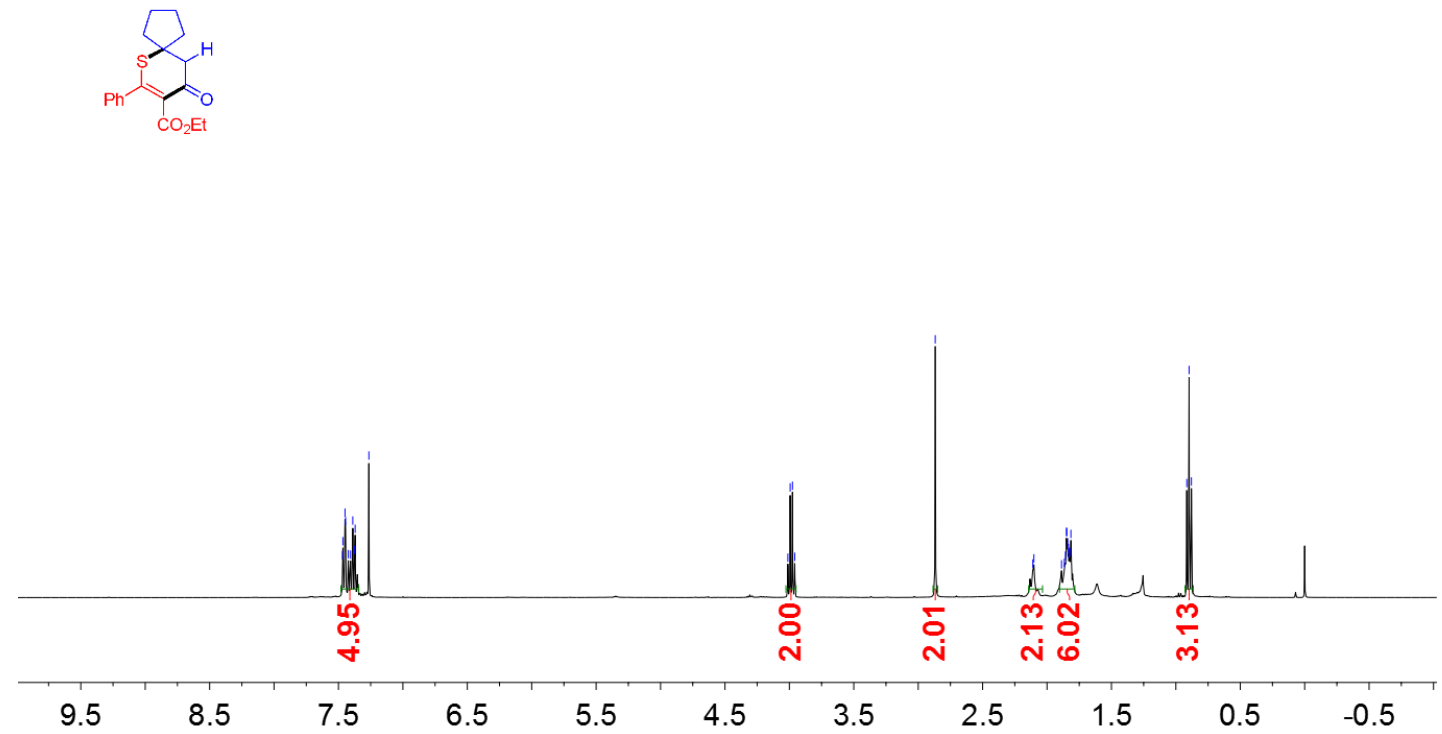

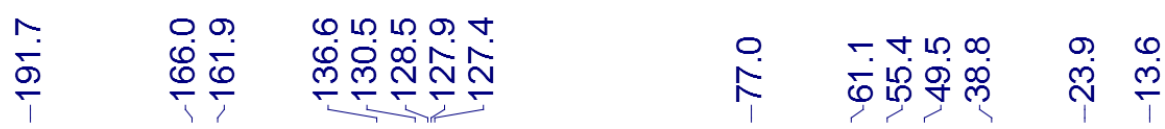
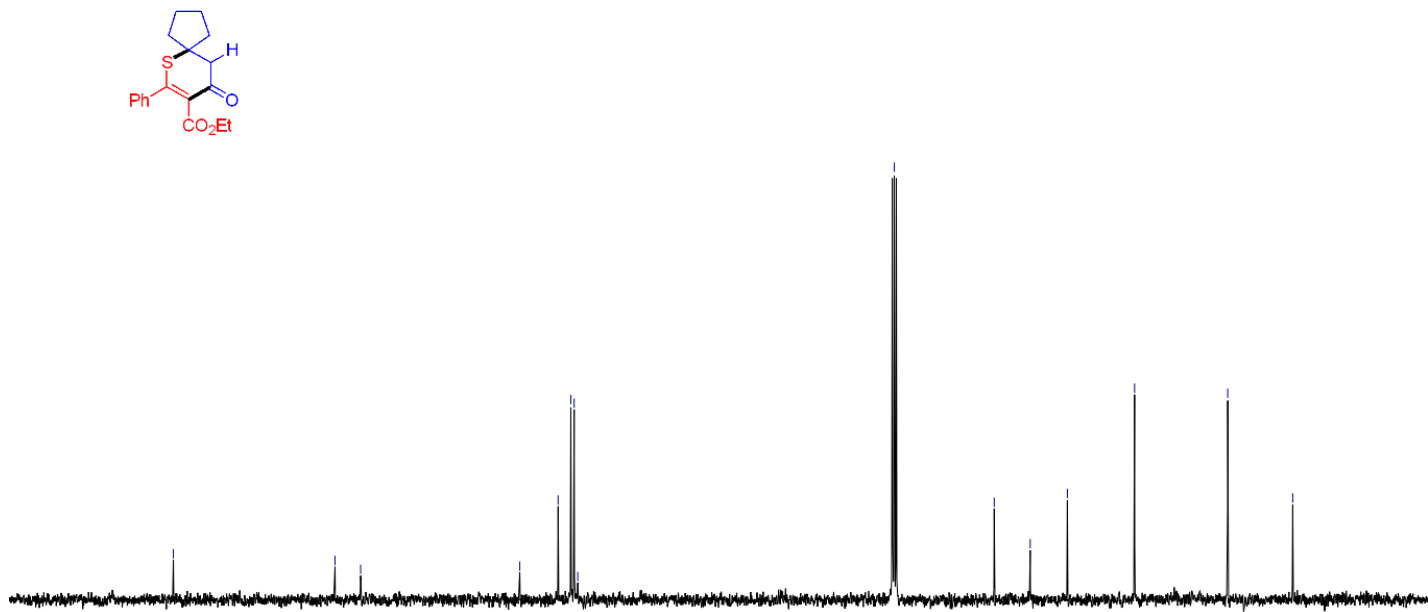

200

180

160

140

120

100

80

60

40

20 
${ }^{1} \mathrm{H}$ and ${ }^{13} \mathrm{C}$ of compound 6 af in $\mathrm{CDCl}_{3}$

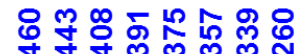

NNNNNN

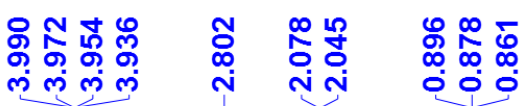
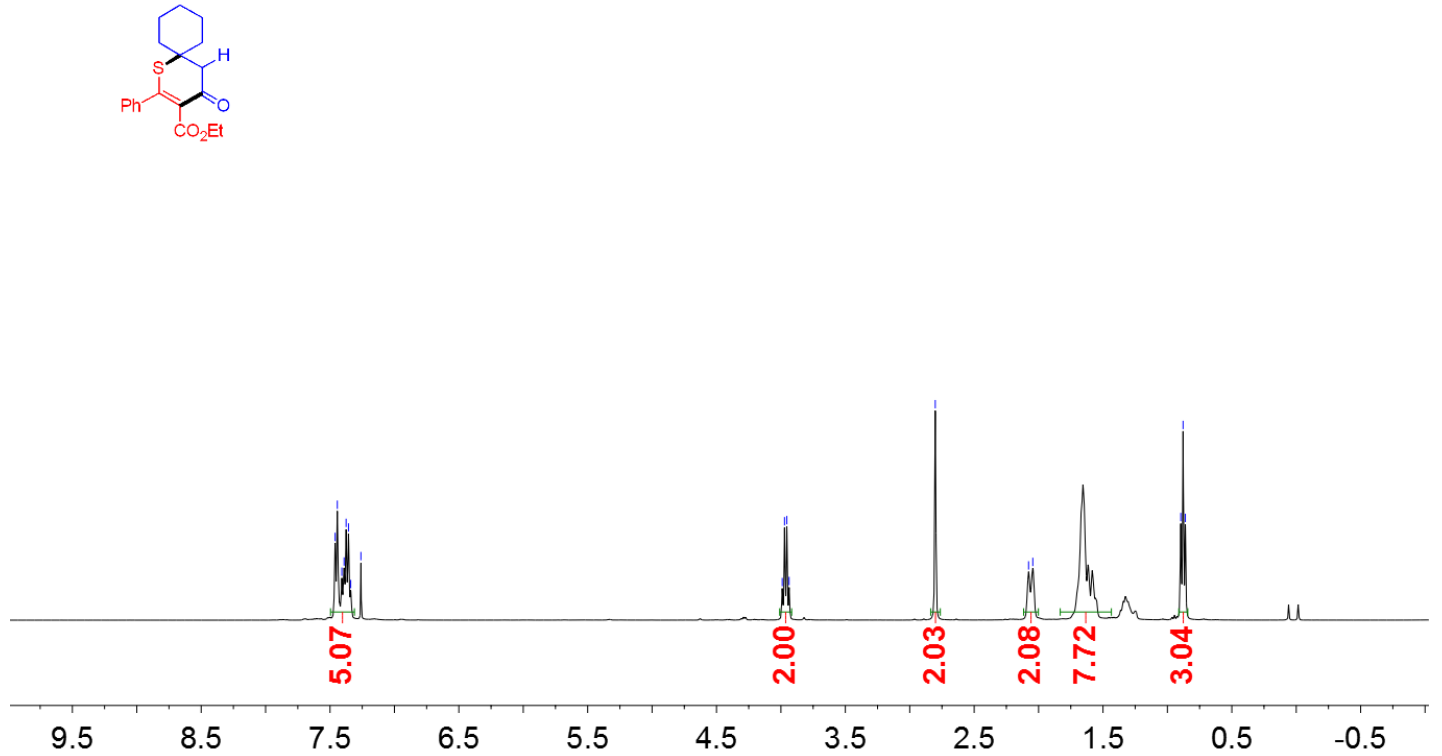

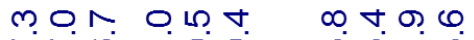

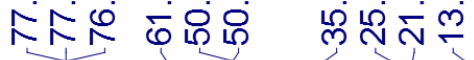

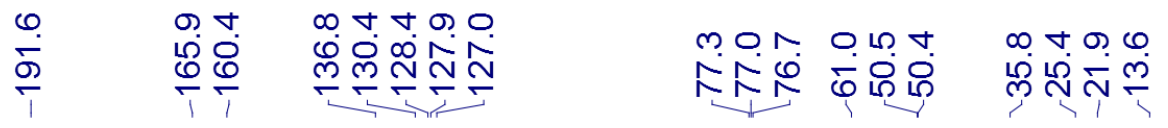
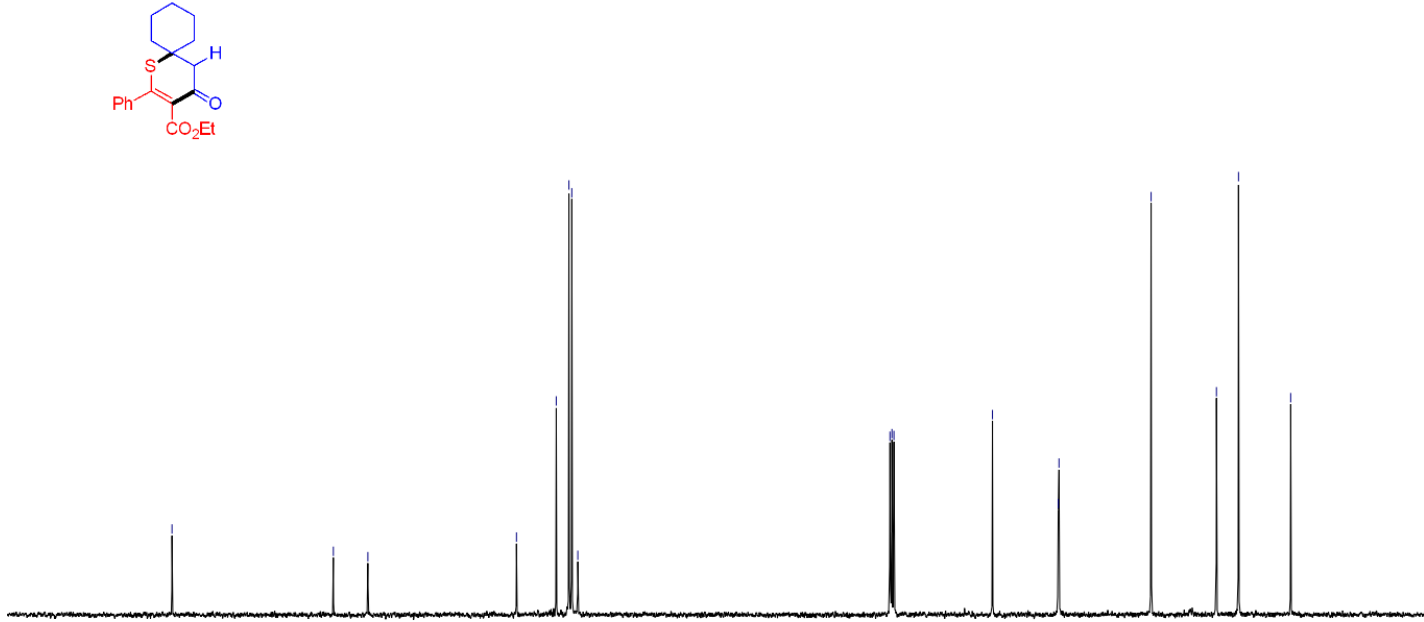

200

180

160

140

120

100

80

60

40

20 
${ }^{1} \mathrm{H}$ and ${ }^{13} \mathrm{C}$ of compound $6 \mathrm{ag}$ in $\mathrm{CDCl}_{3}$

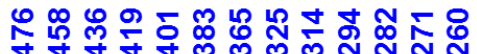

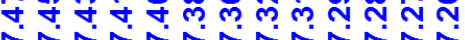

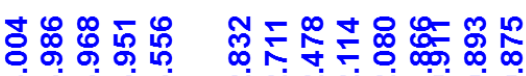

نेंm m
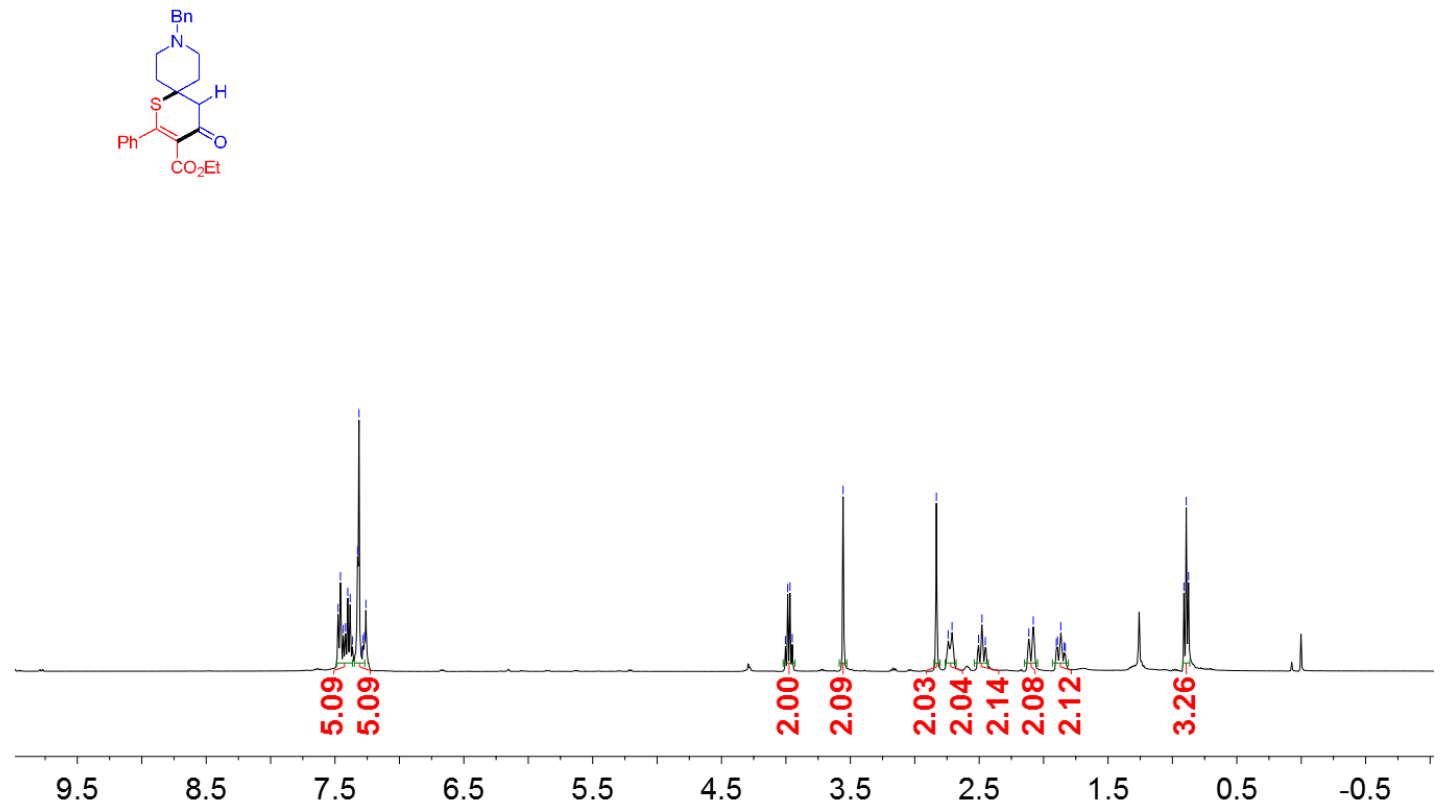

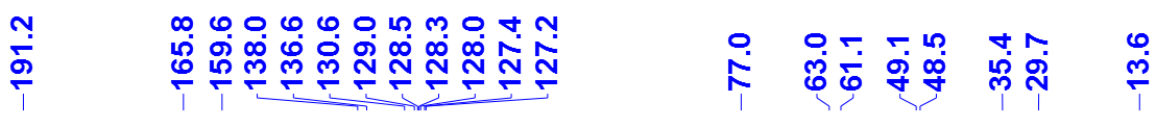
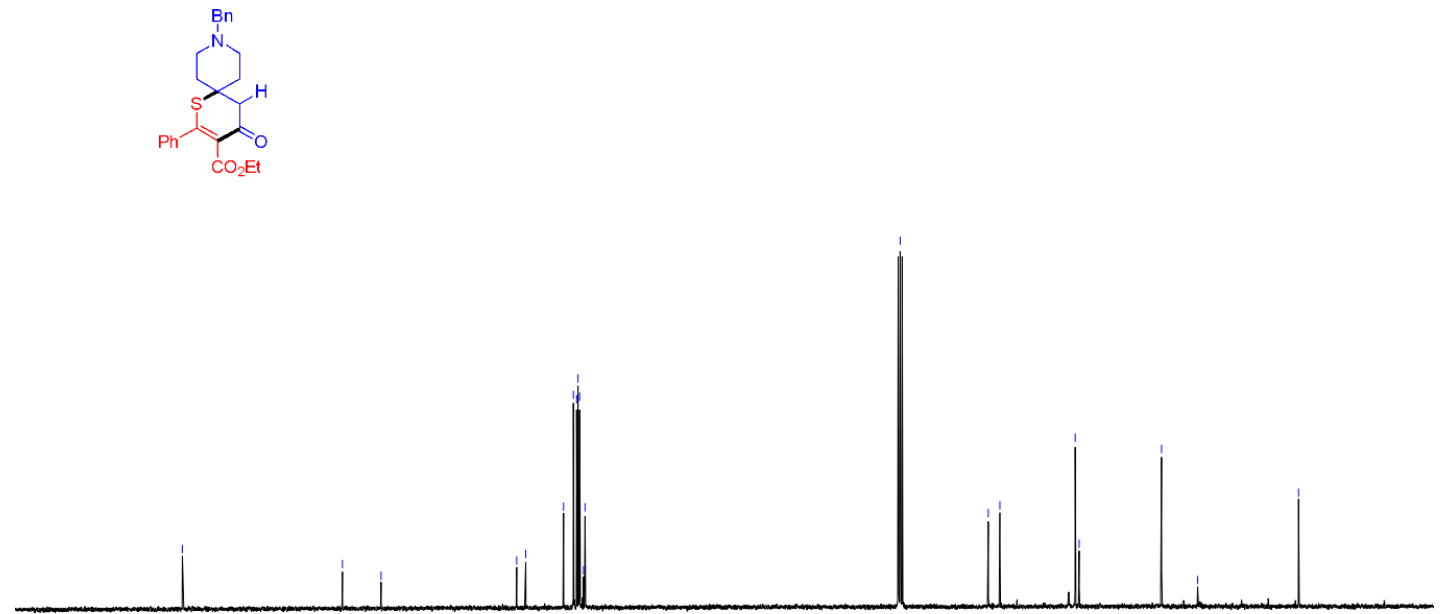

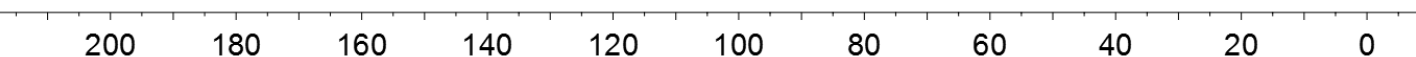


${ }^{1} \mathrm{H}$ and ${ }^{13} \mathrm{C}$ of compound $6 \mathrm{ah}$ in $\mathrm{CDCl}_{3}$

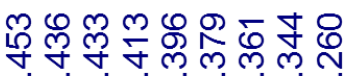
NNNNNNN

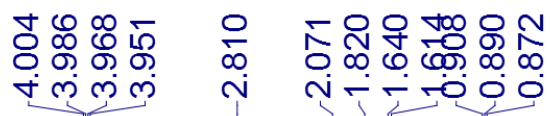
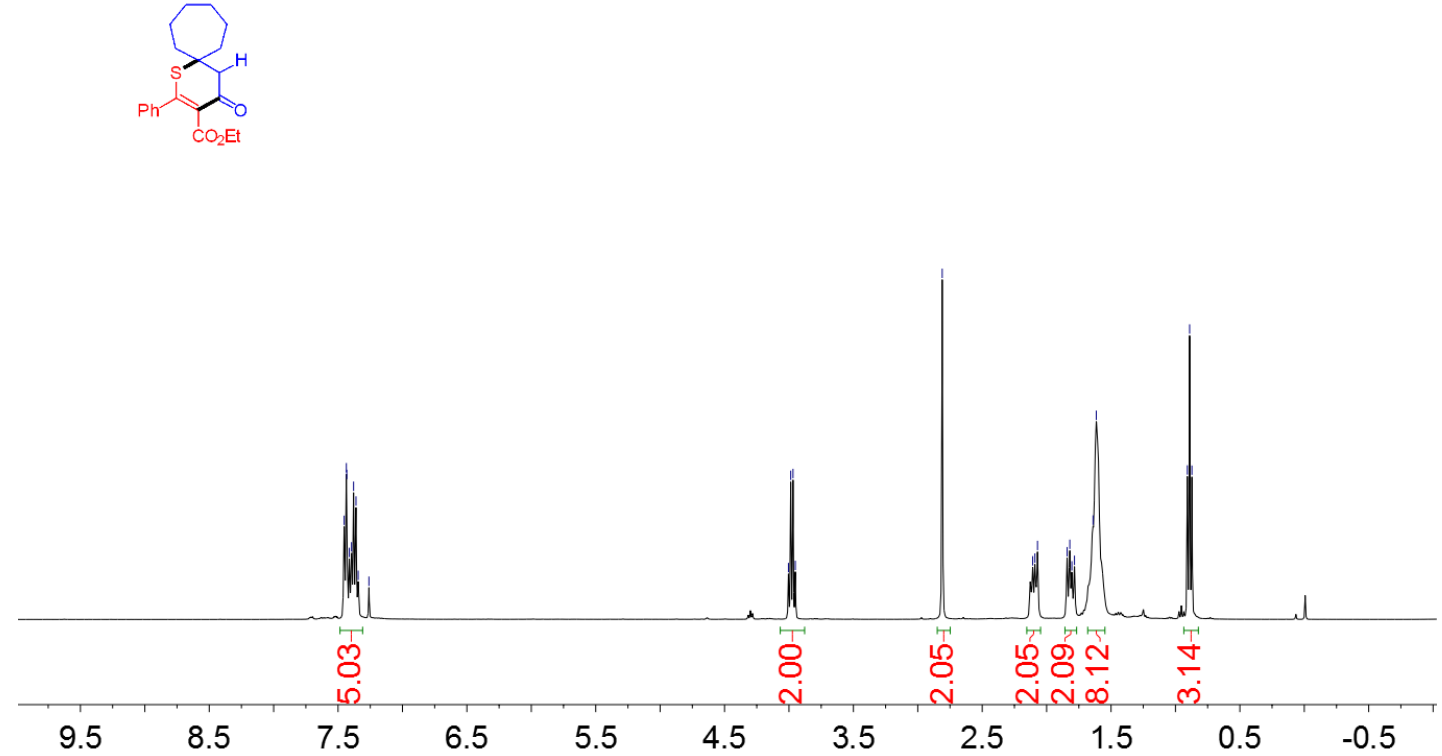

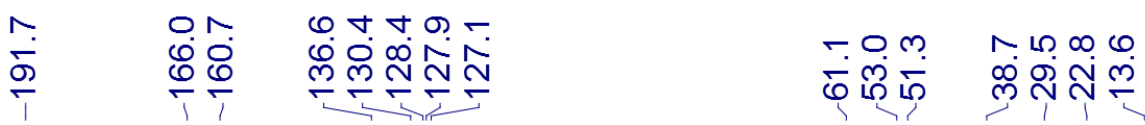
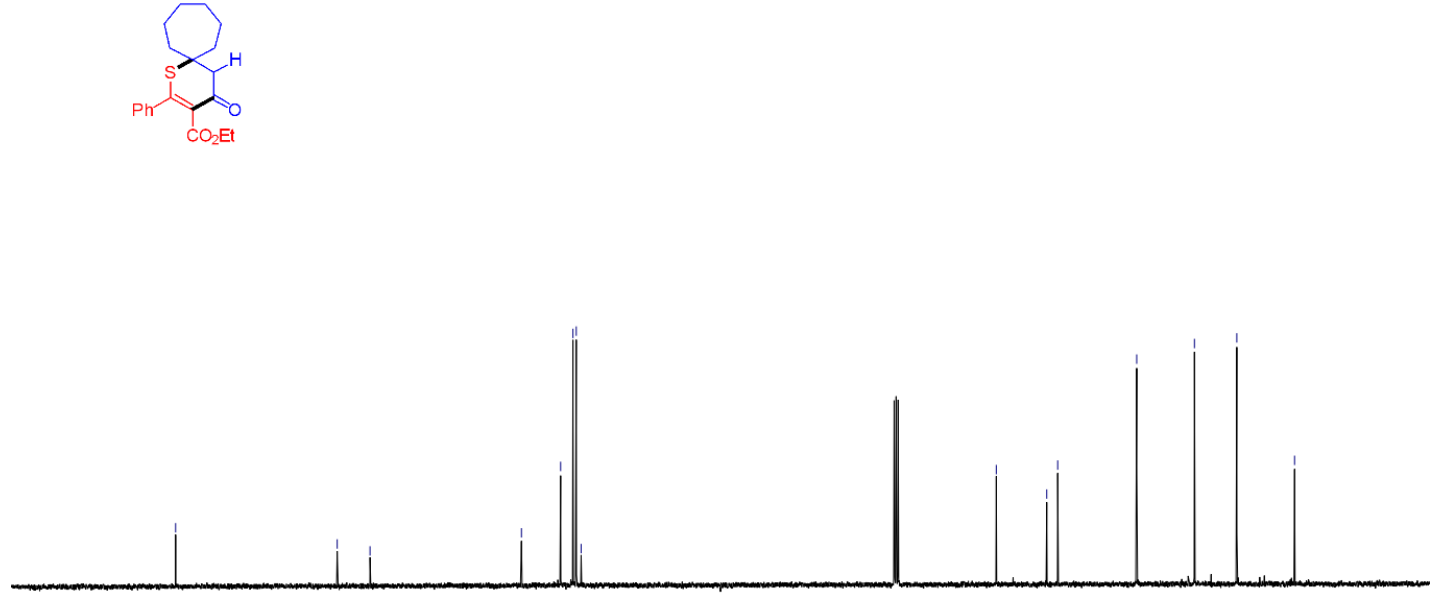

200 18 160 140 120 100 80

60

40

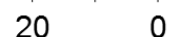


${ }^{1} \mathrm{H}$ and ${ }^{13} \mathrm{C}$ of compound 6ai in $\mathrm{CDCl}_{3}$

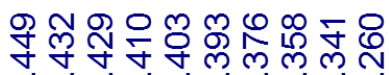
NNNNNNNN

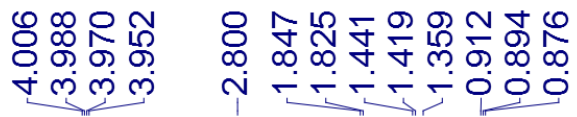<smiles>CCCCCCCCCC1SC(C)=C(C(=O)OCC)C1CCC</smiles>

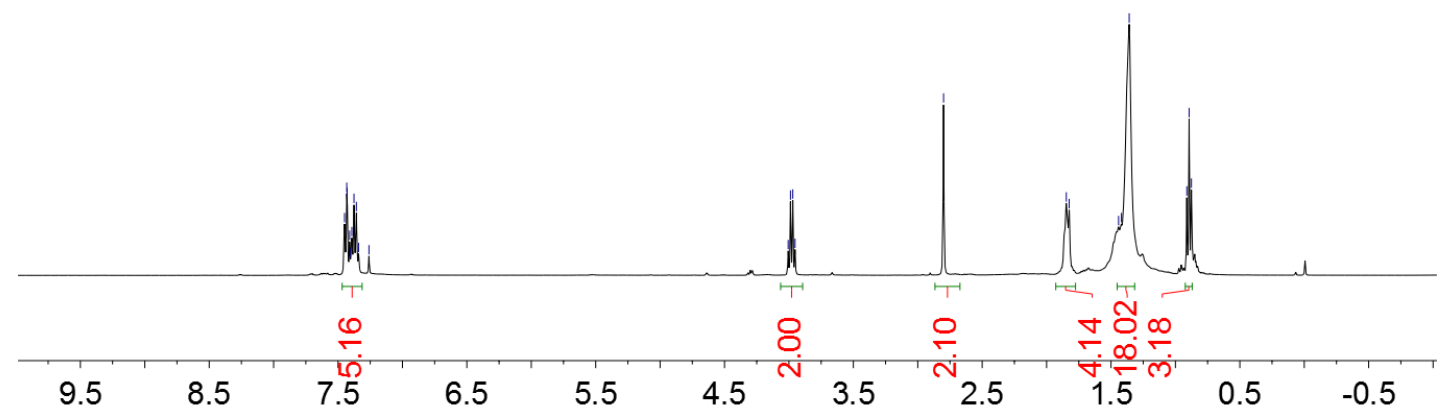

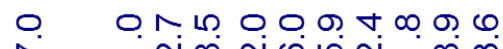
N $\quad$ ธิกัน

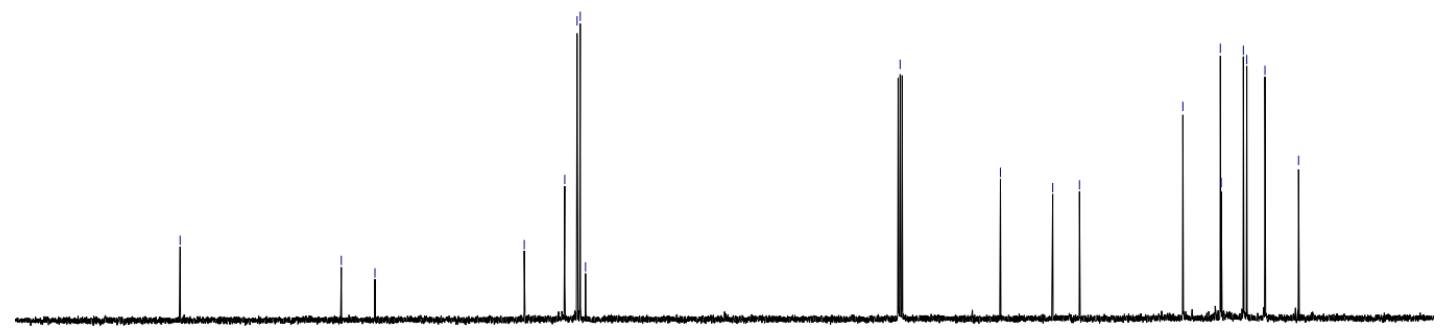

200 180 160 140 120 100 80 20 
${ }^{1} \mathrm{H}$ and ${ }^{13} \mathrm{C}$ of compound 6aj in $\mathrm{CDCl}_{3}$

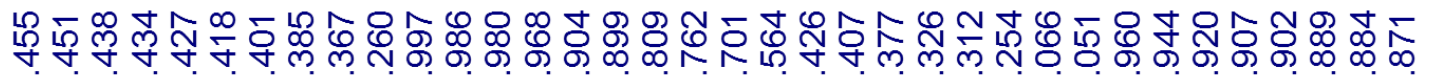

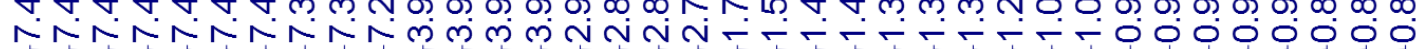
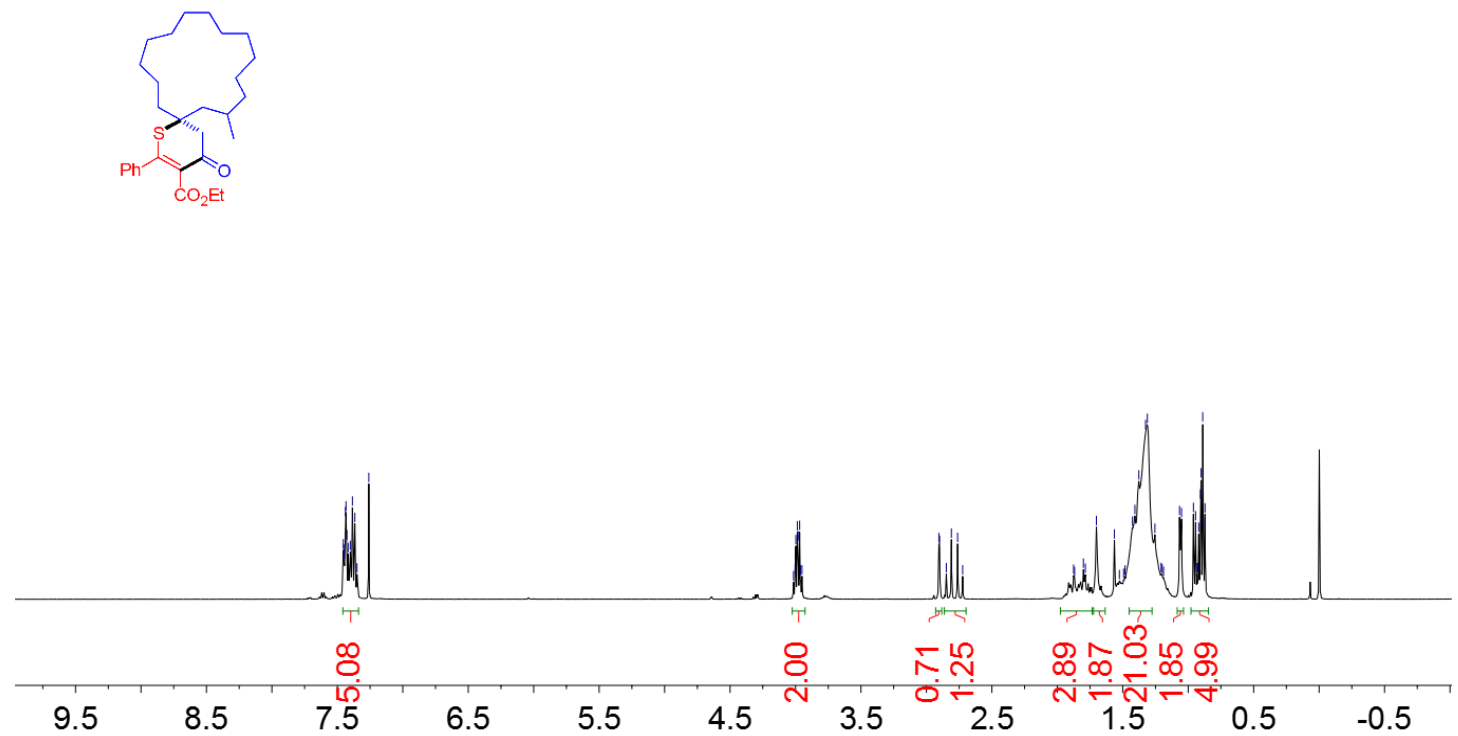

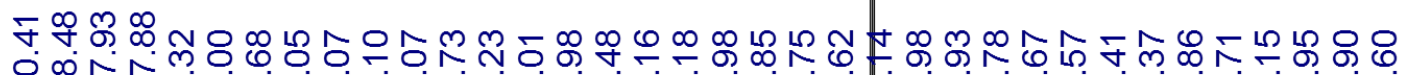
m
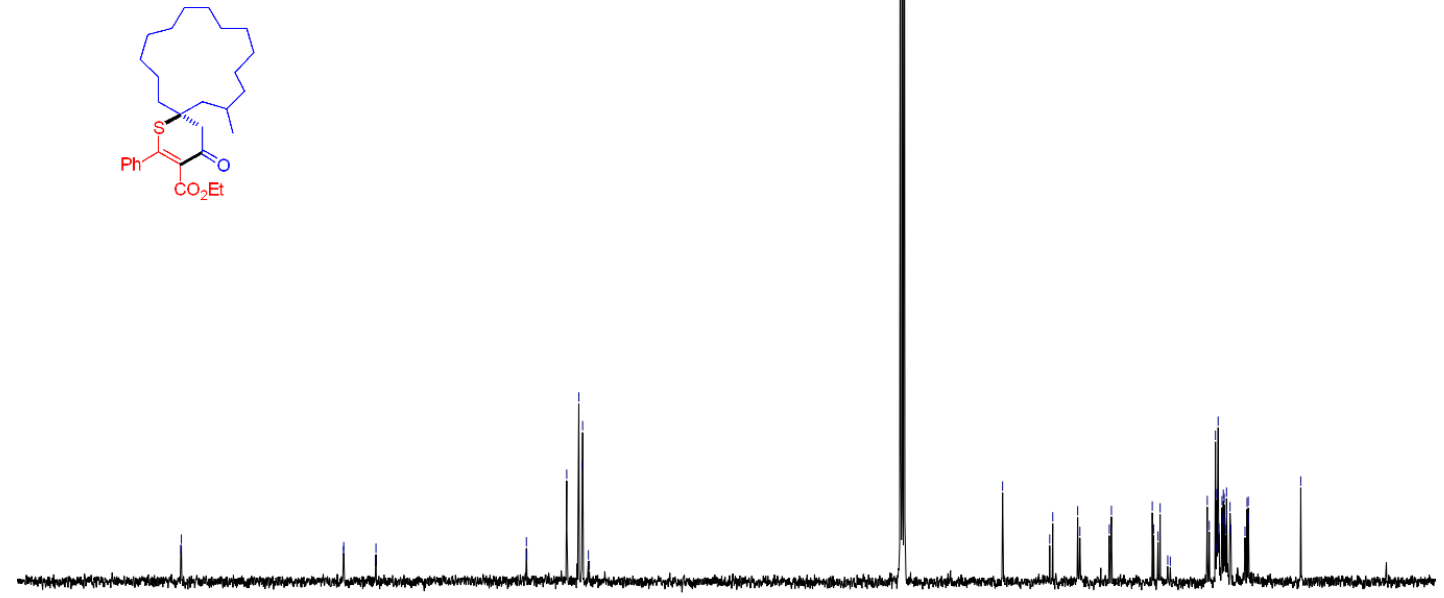

200 180 160 140 120 100

80

60

40 
${ }^{1} \mathrm{H}$ and ${ }^{13} \mathrm{C}$ of compound 6 ak in $\mathrm{CDCl}_{3}$

寸̛ 舟 N N N N N N N
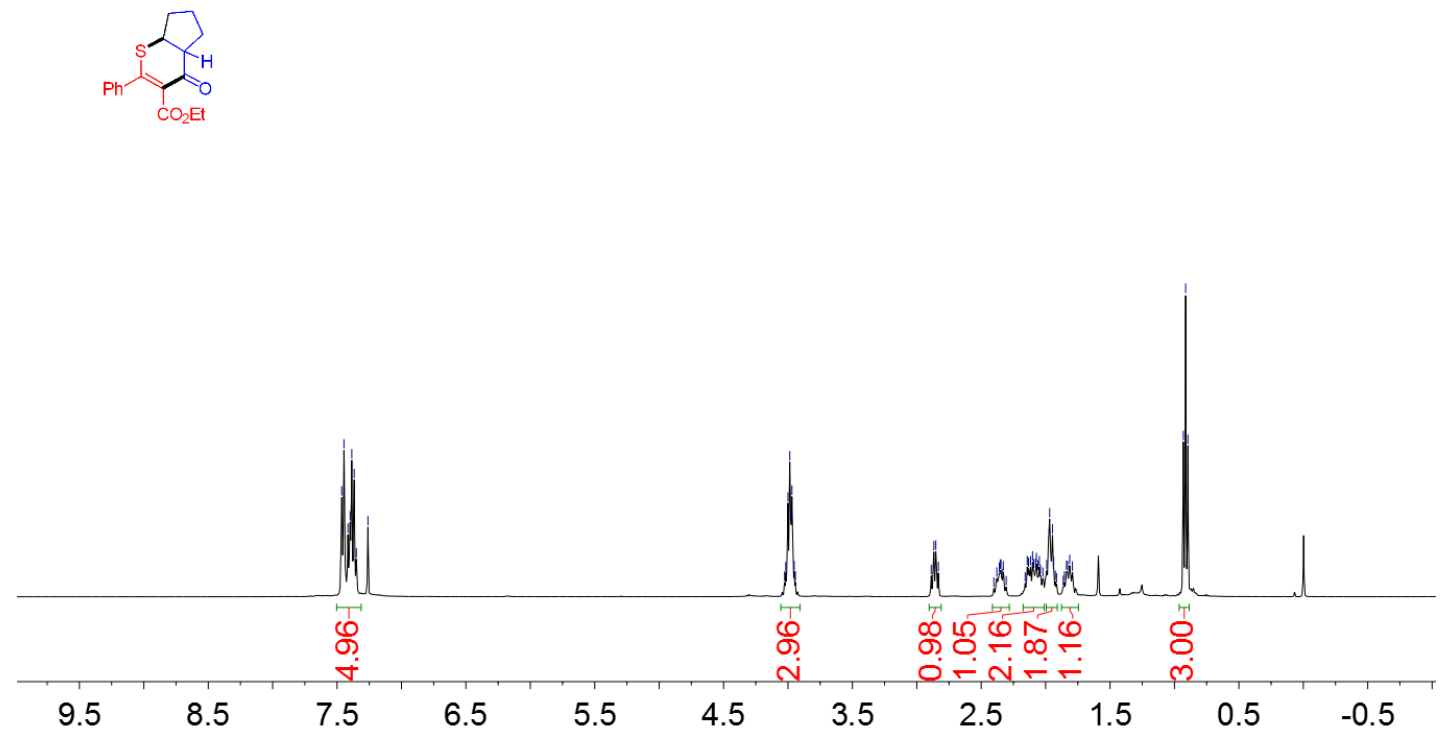

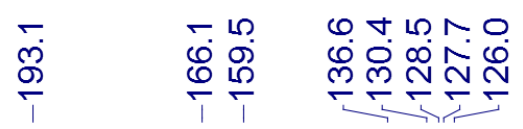

m.N Nọ. N.

NN⿱
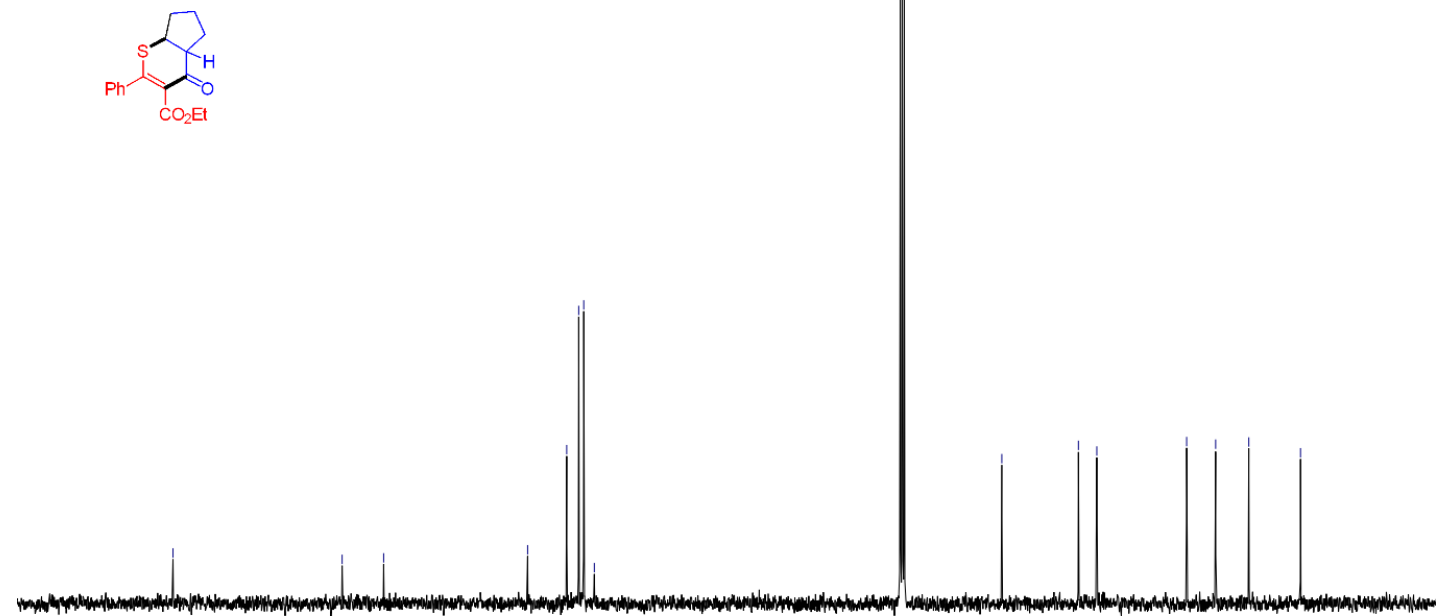

200

180

160

140

120

100

80

60

40

20 
${ }^{1} \mathrm{H}$ and ${ }^{13} \mathrm{C}$ of compound $4 \mathrm{ba}$ in $\mathrm{CDCl}_{3}$

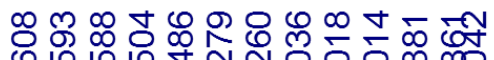

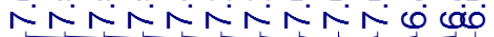

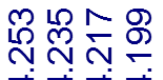

N

守守文

テ
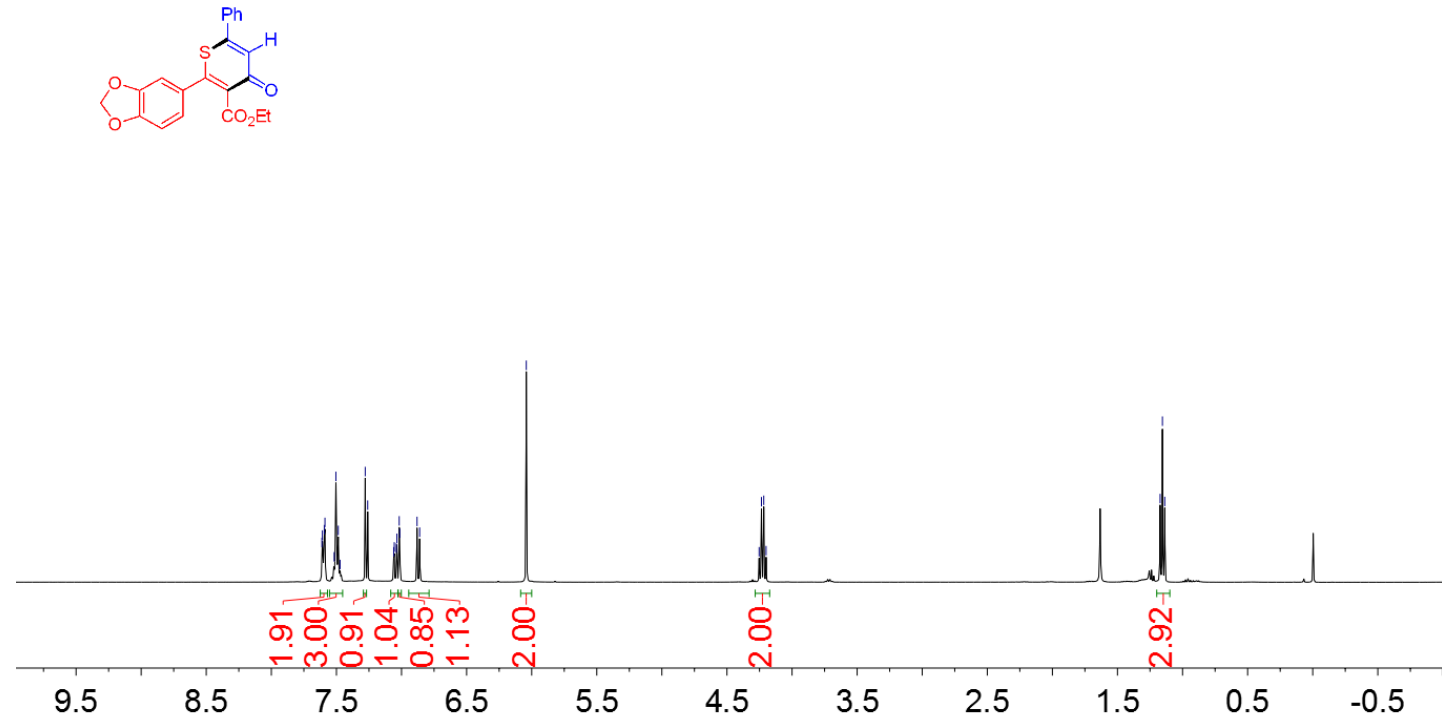

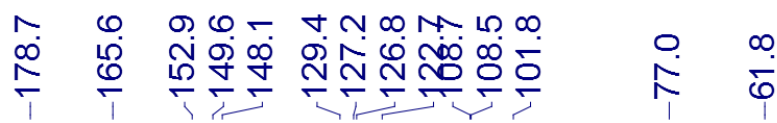

$\stackrel{\infty}{\frac{m}{1}}$

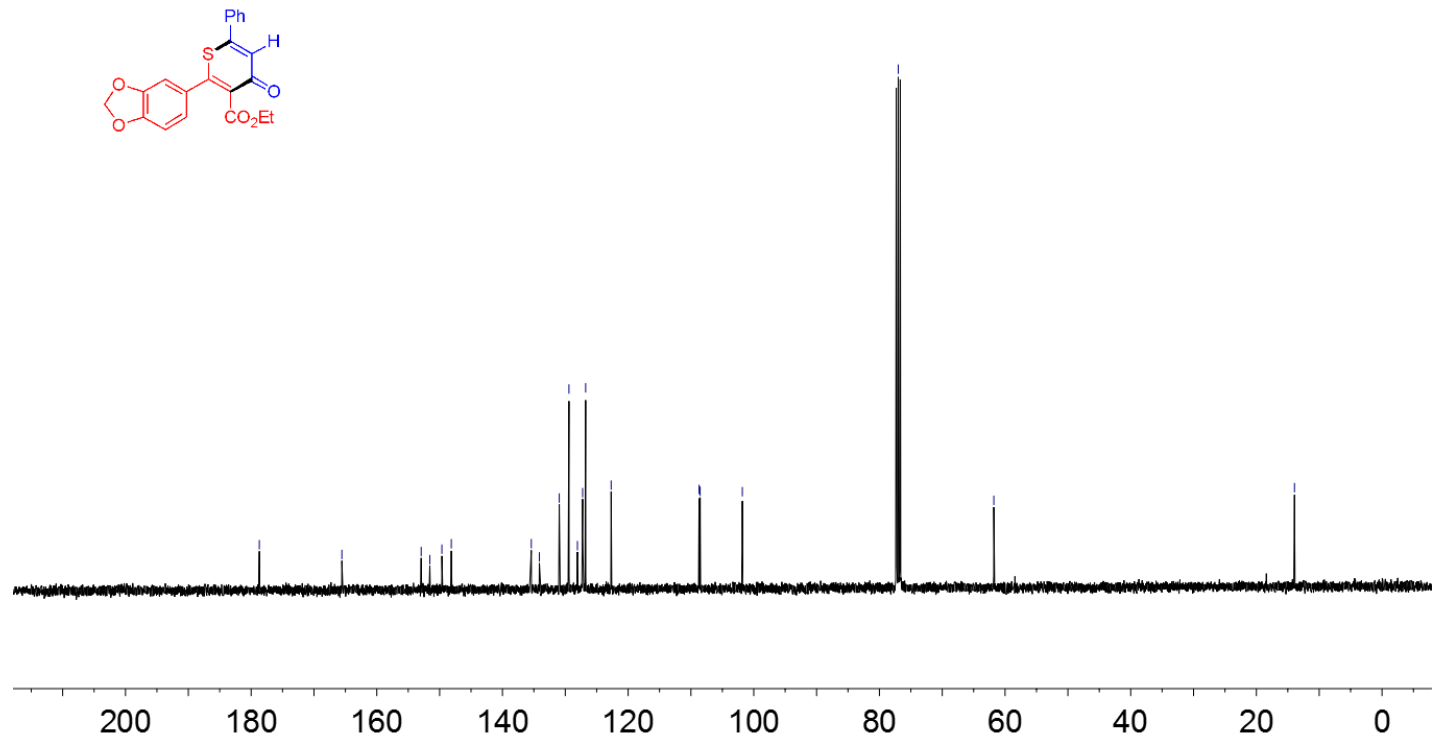


${ }^{1} \mathrm{H}$ and ${ }^{13} \mathrm{C}$ of compound $4 \mathrm{ca}$ in $\mathrm{CDCl}_{3}$

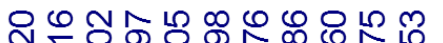

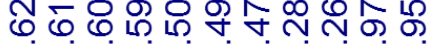

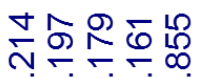

ํํำ

NNNNNNNG6

守守守
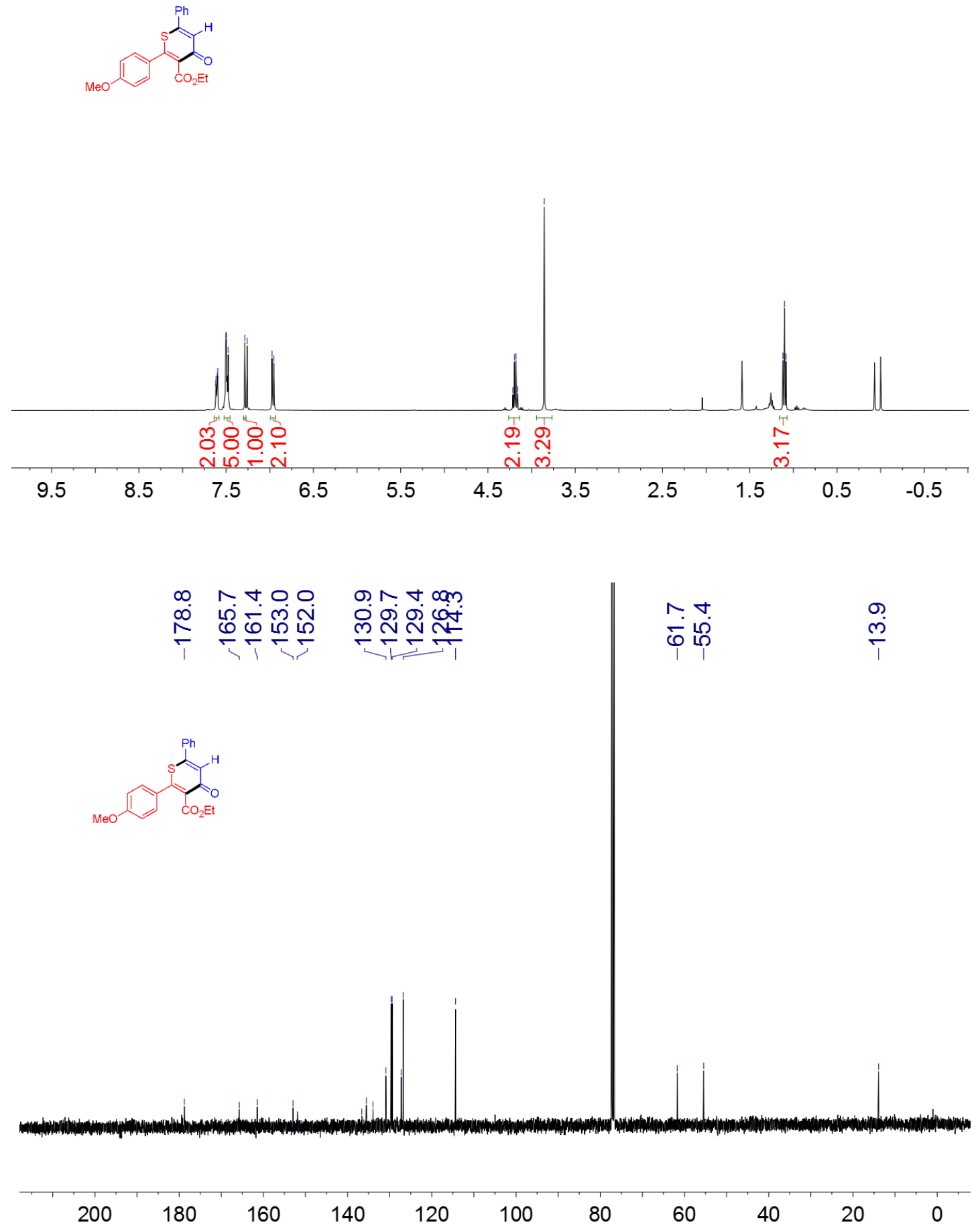
${ }^{1} \mathrm{H}$ and ${ }^{13} \mathrm{C}$ of compound $4 \mathrm{da}$ in $\mathrm{CDCl}_{3}$

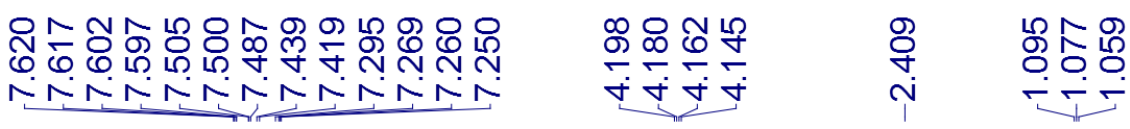
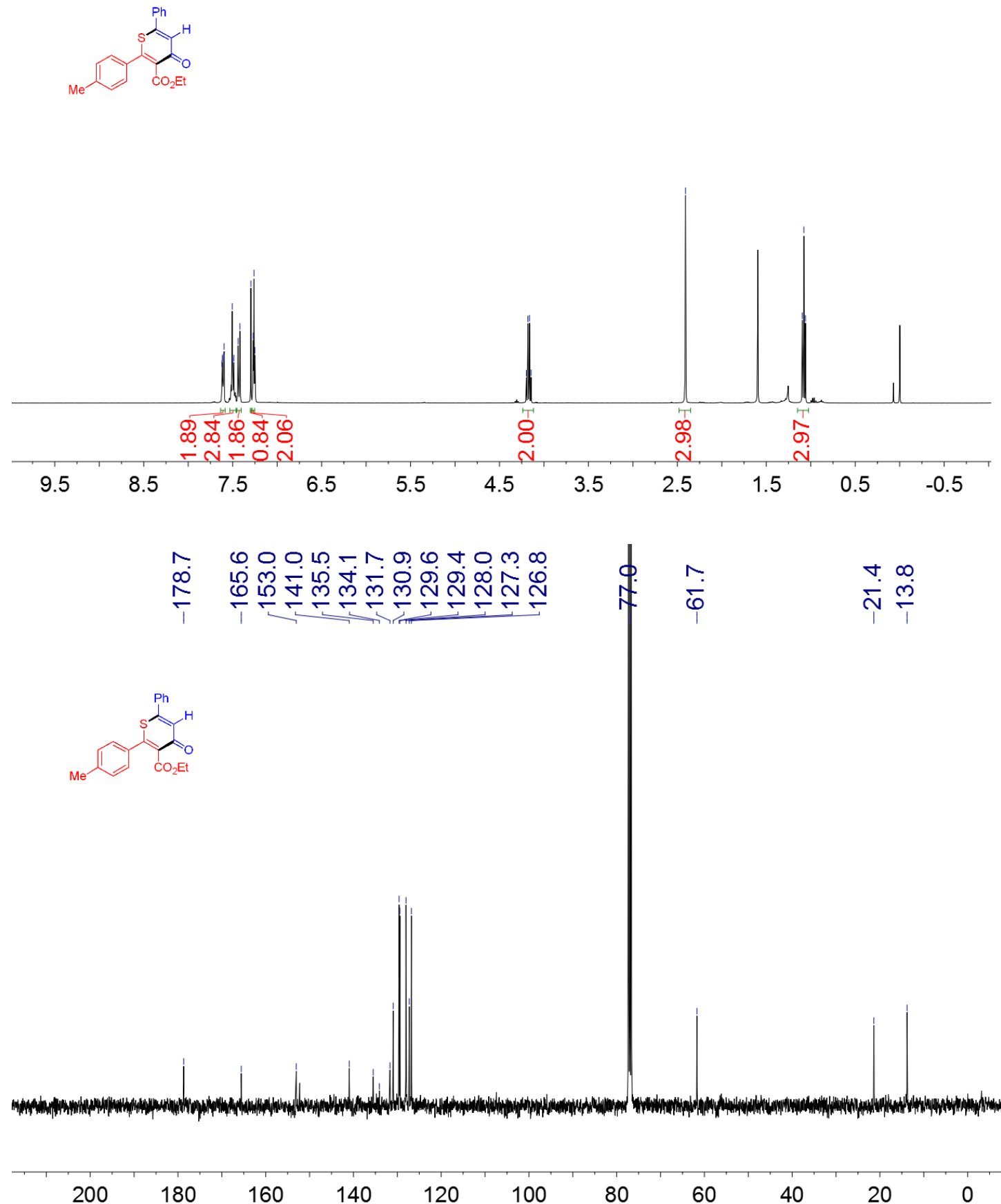
${ }^{1} \mathrm{H}{ }^{13} \mathrm{C}$ and ${ }^{19} \mathrm{~F}$ of compound 4ea in $\mathrm{CDCl}_{3}$

\begin{tabular}{|c|c|c|}
\hline 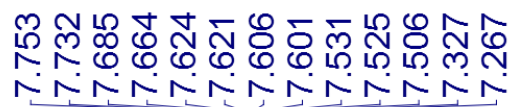 & 我 & 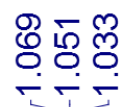 \\
\hline
\end{tabular}
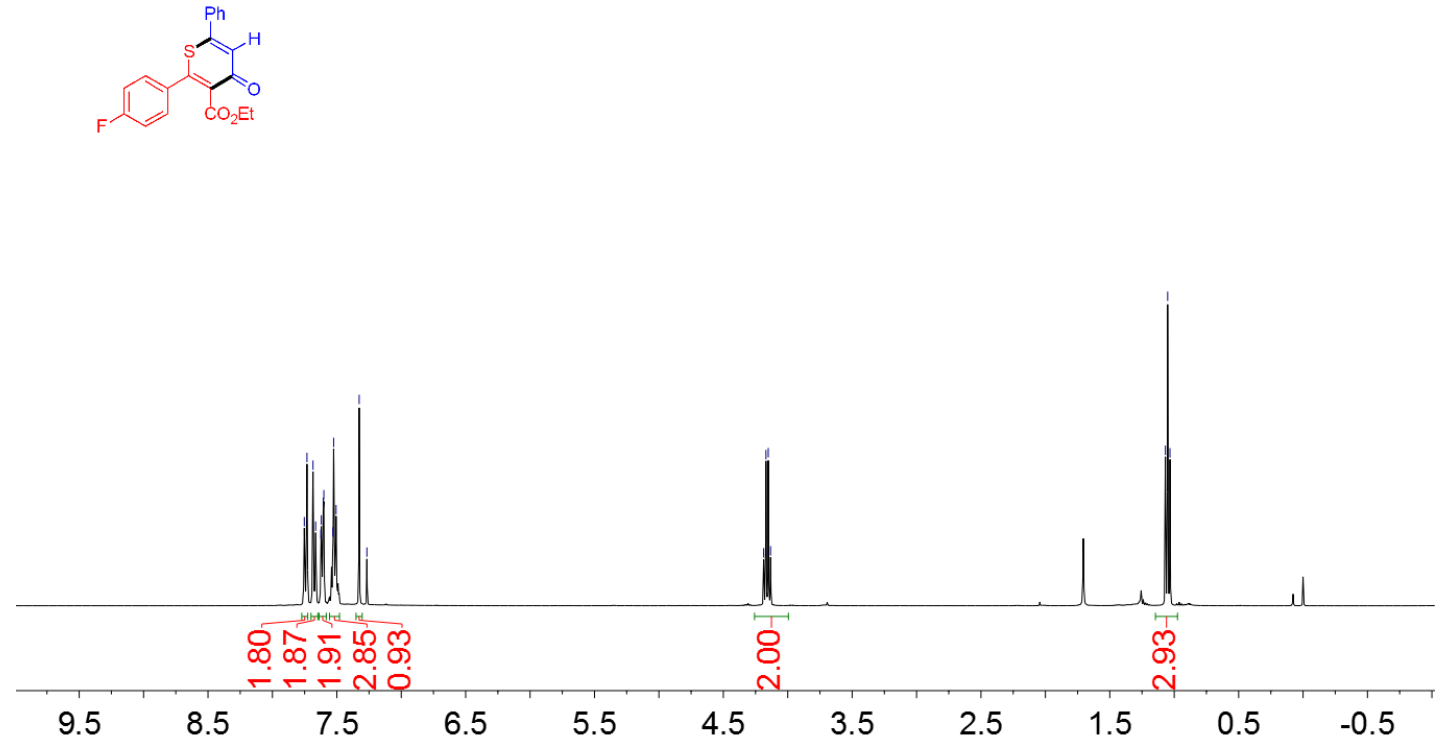

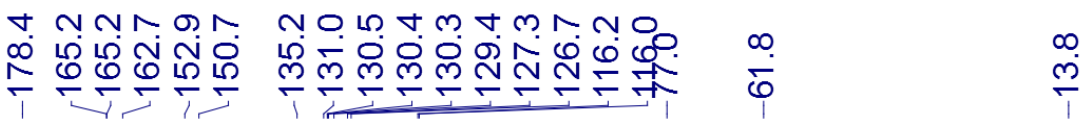

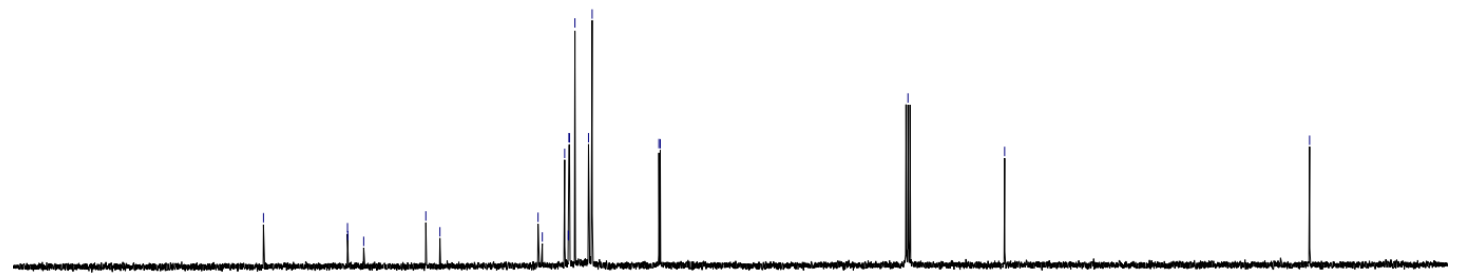

200

180

160

140

120

100

80

40

20 


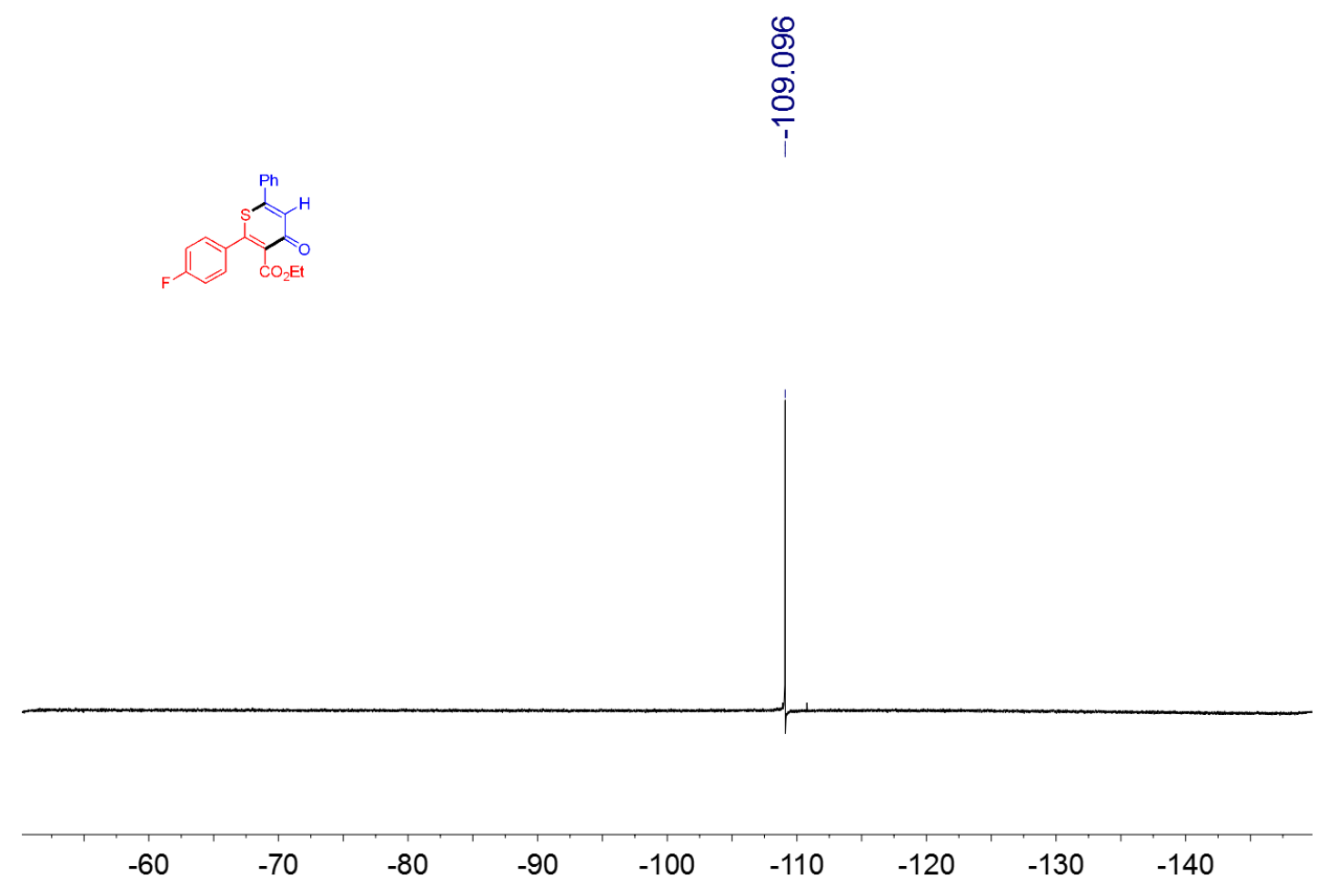


${ }^{1} \mathrm{H}$ and ${ }^{13} \mathrm{C}$ of compound $4 \mathrm{fa}$ in $\mathrm{CDCl}_{3}$

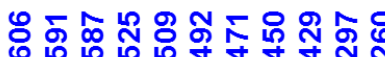

NNNNNNNN

冚

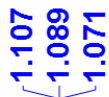
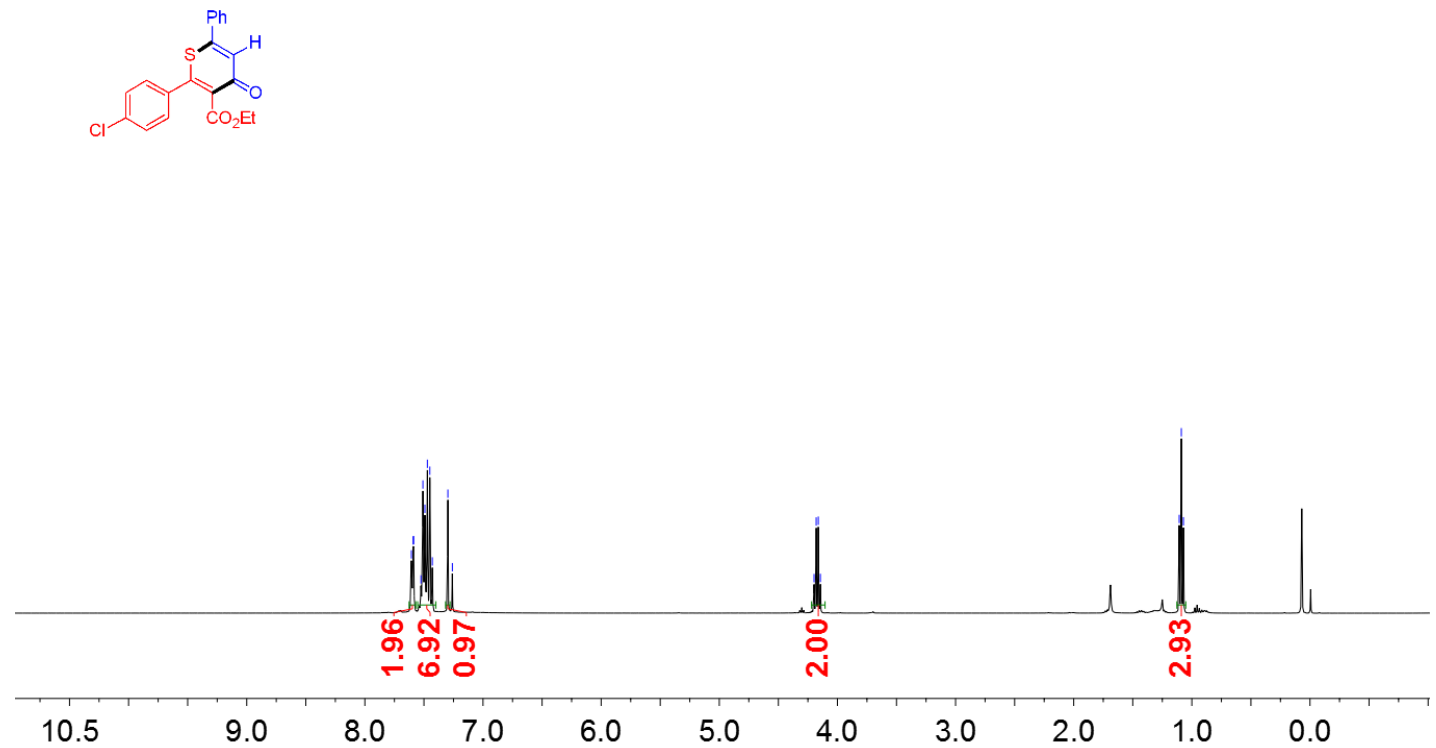

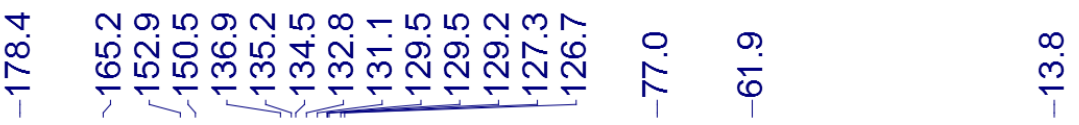

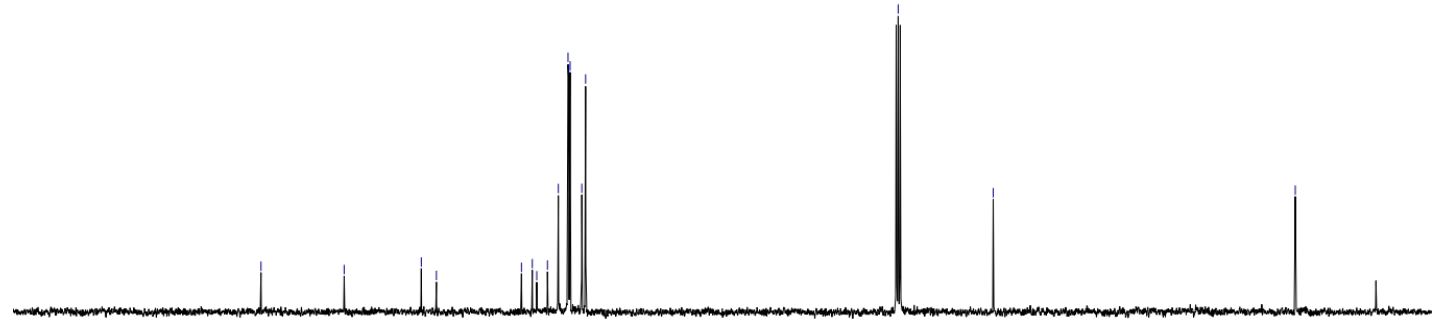

200

180

160

140

120

100

80

6

20 
${ }^{1} \mathrm{H}$ and ${ }^{13} \mathrm{C}$ of compound $4 \mathrm{ga}$ in $\mathrm{CDCl}_{3}$ ๘

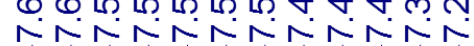
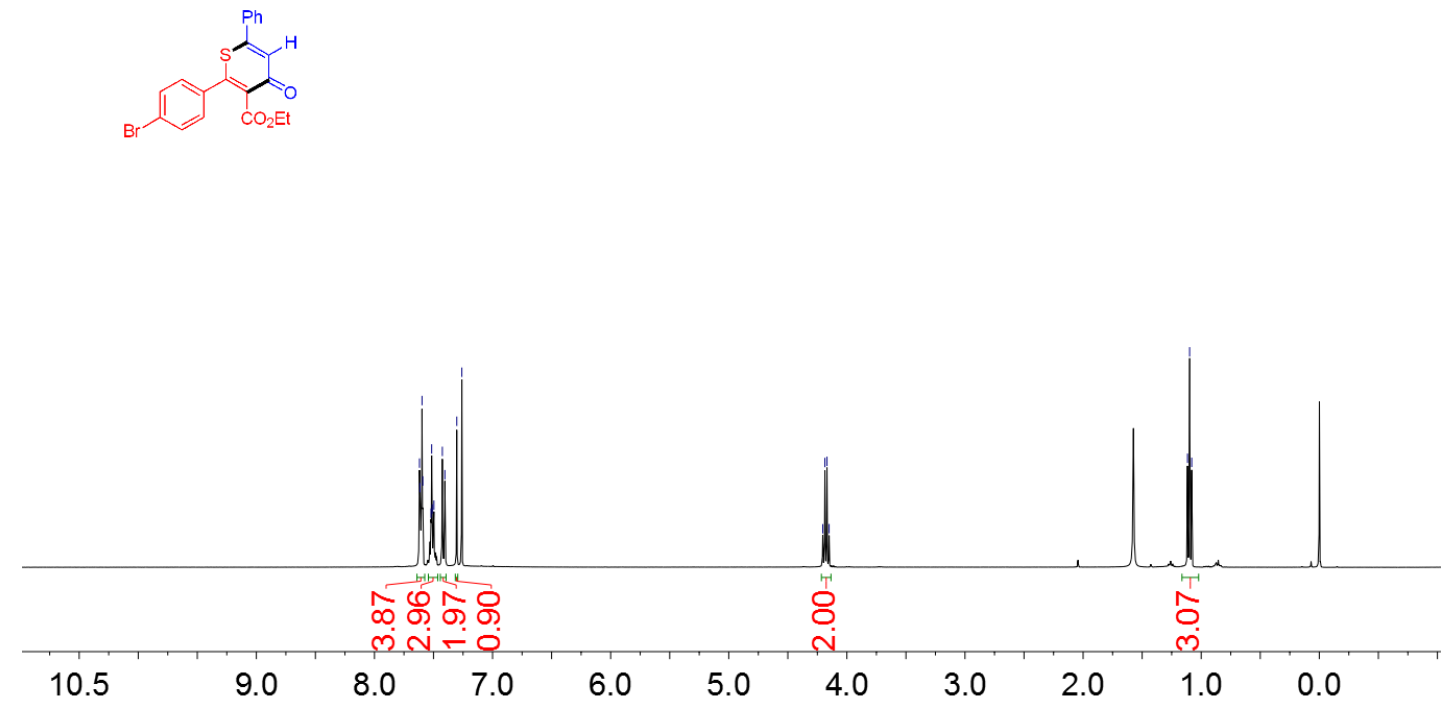

ป. $\quad$ ก

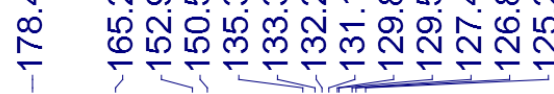

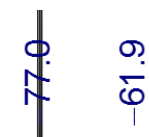

$\stackrel{\infty}{\stackrel{\infty}{\Gamma}}$

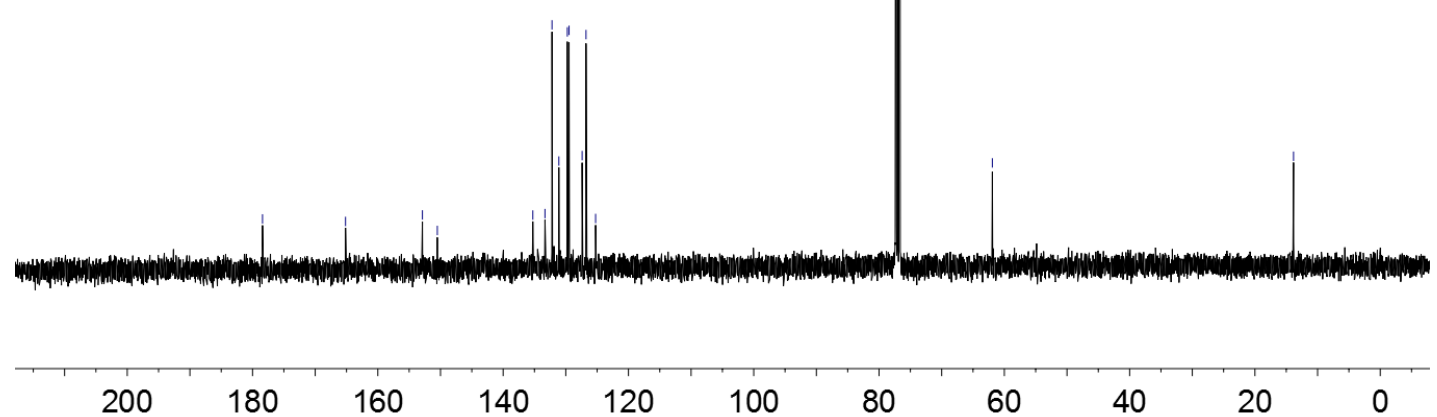


${ }^{1} \mathrm{H}$ and ${ }^{13} \mathrm{C}$ of compound 4 ha in $\mathrm{CDCl}_{3}$

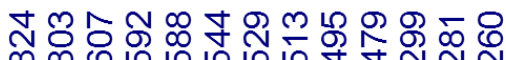

กุ๊

요

守守守

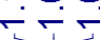
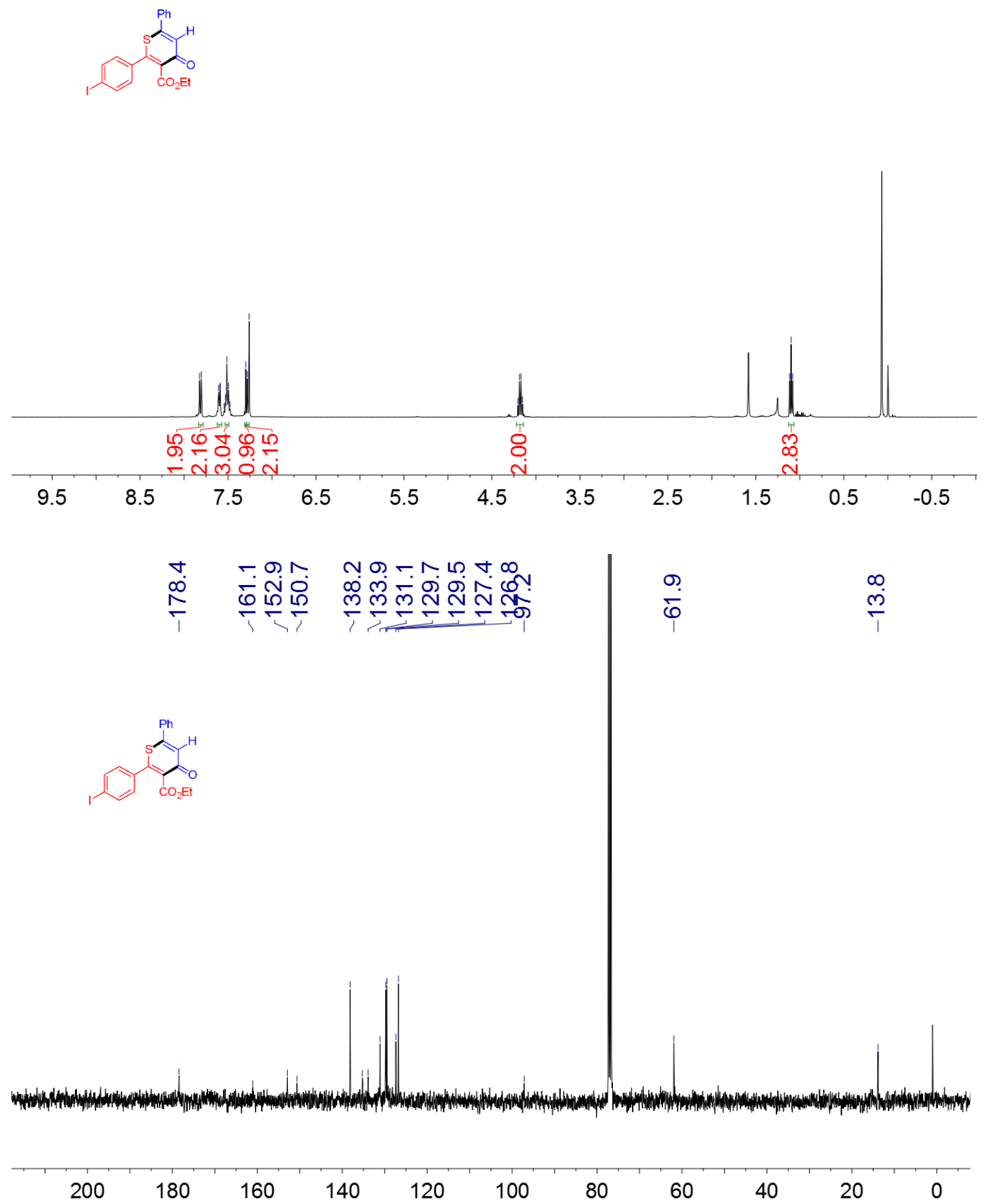
${ }^{1} \mathrm{H}$ and ${ }^{13} \mathrm{C}$ of compound $4 \mathrm{ia}$ in $\mathrm{CDCl}_{3}$

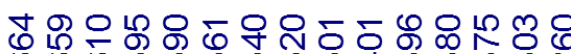
NNNNNNNNNNN

ํํำ ำ

N
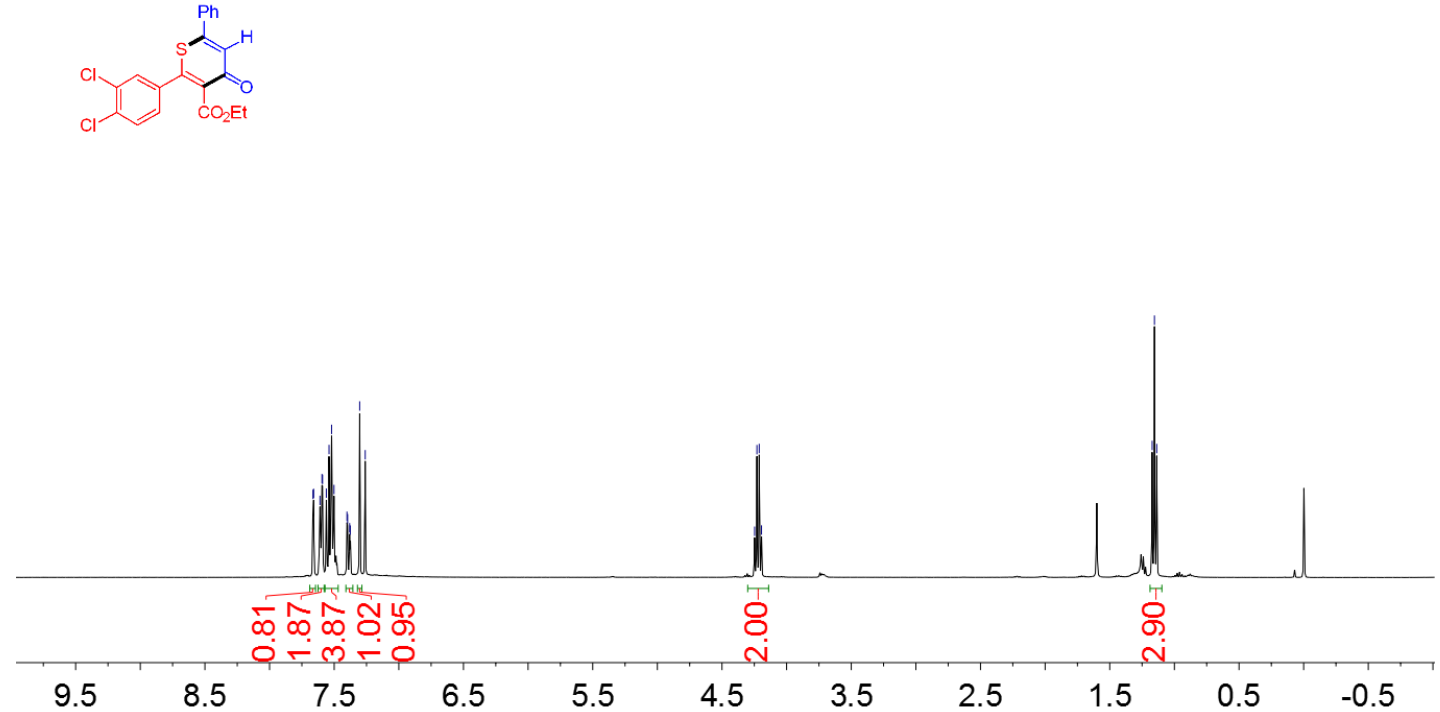

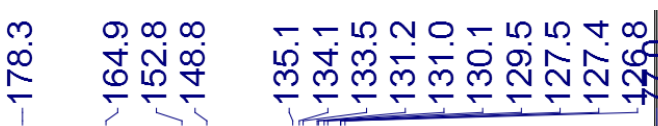

กิ่

$\stackrel{9}{\square}$
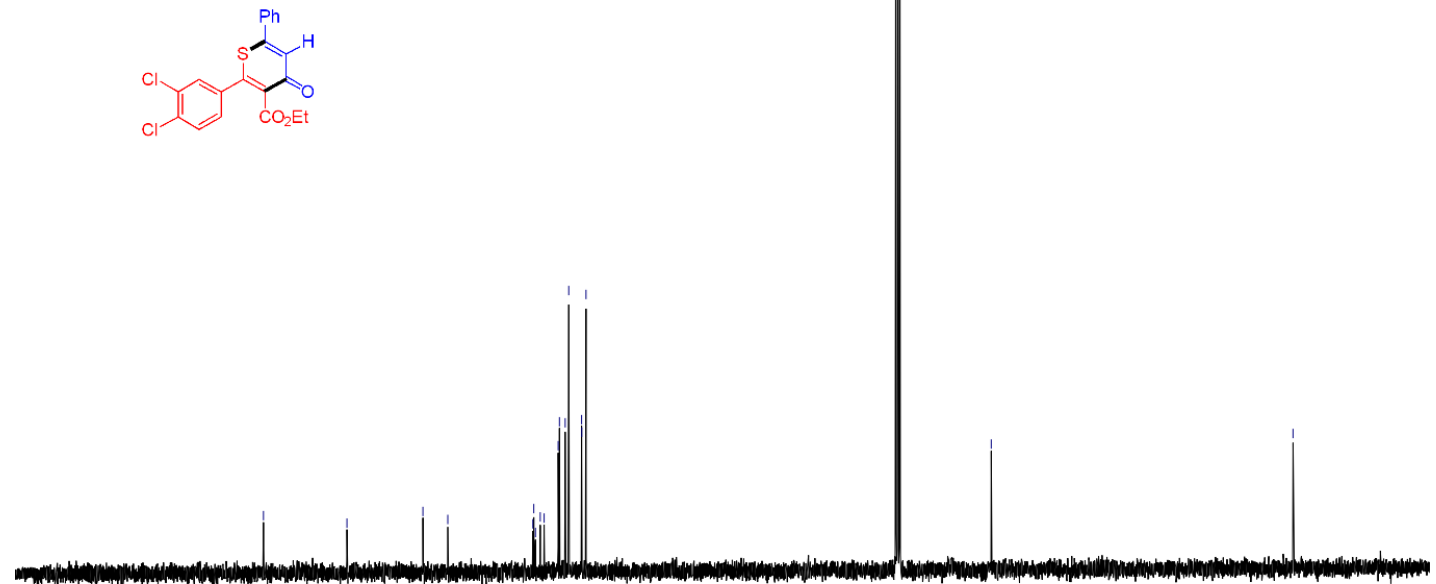

200 
${ }^{1} \mathrm{H}$ and ${ }^{13} \mathrm{C}$ of compound $4 \mathrm{ja}$ in $\mathrm{CDCl}_{3}$

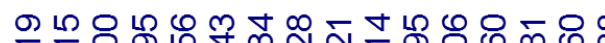

६ 6 ถ

NNNNNNNNNNNNNN

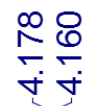

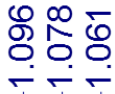
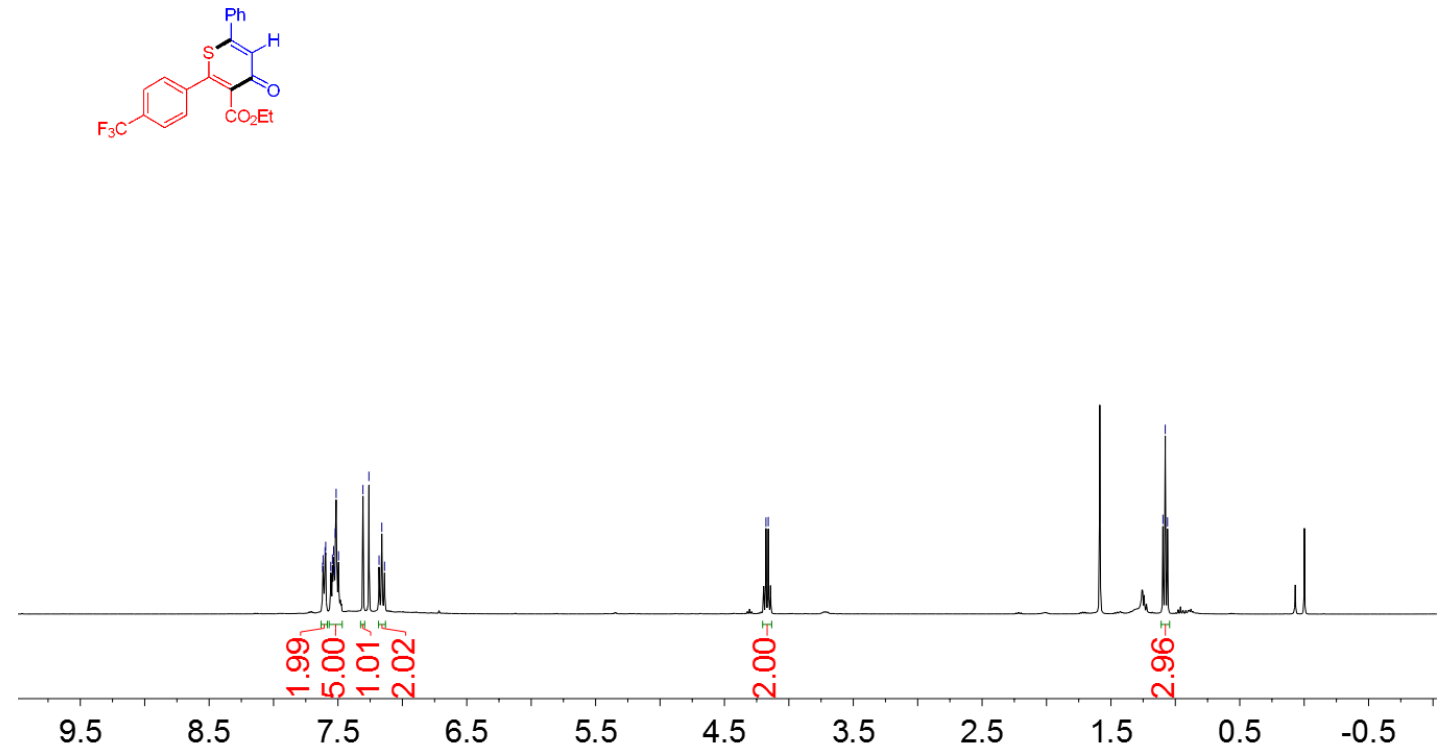

ก

ำ

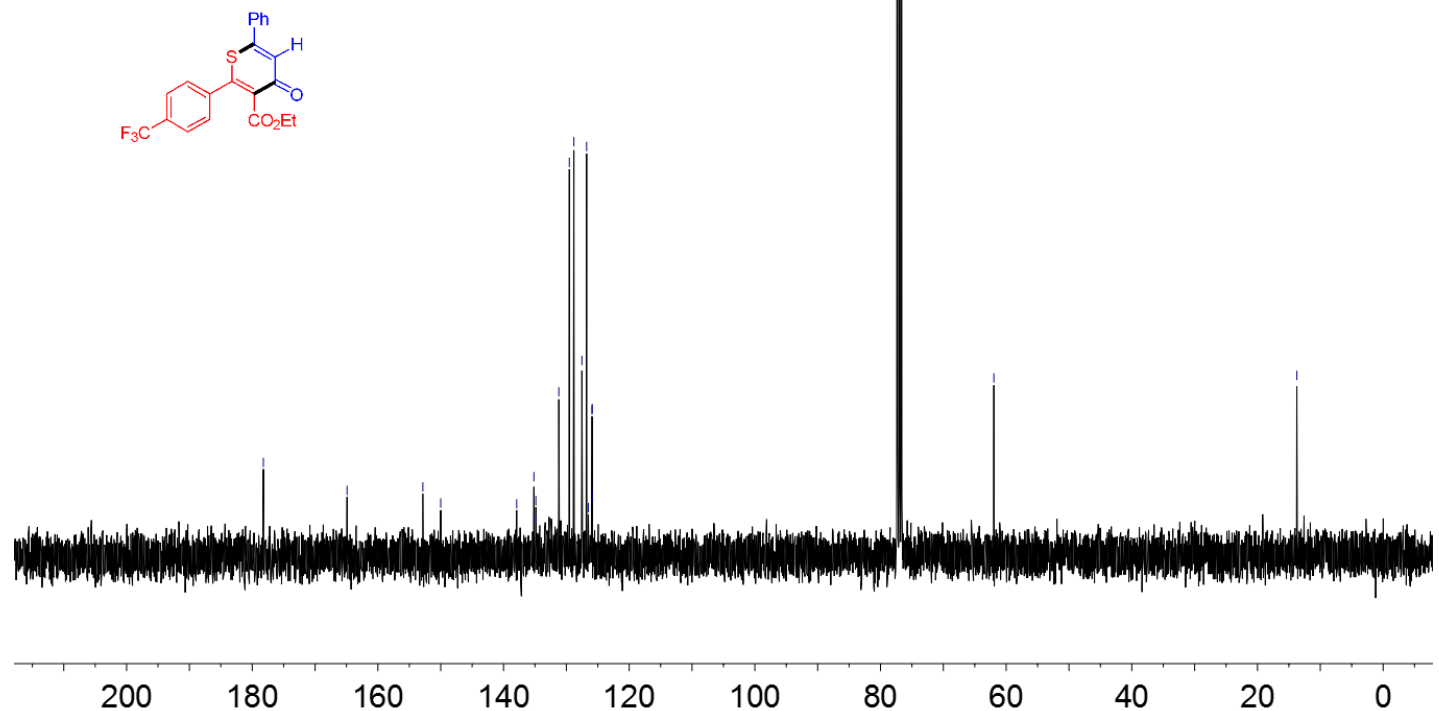


${ }^{1} \mathrm{H}$ and ${ }^{13} \mathrm{C}$ of compound $4 \mathrm{ka}$ in $\mathrm{CDCl}_{3}$

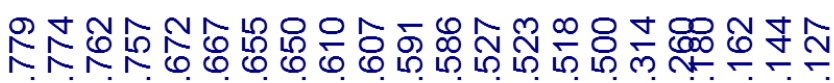

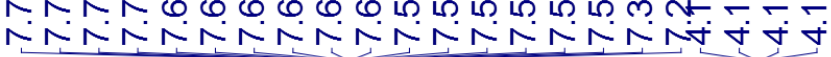

느요용
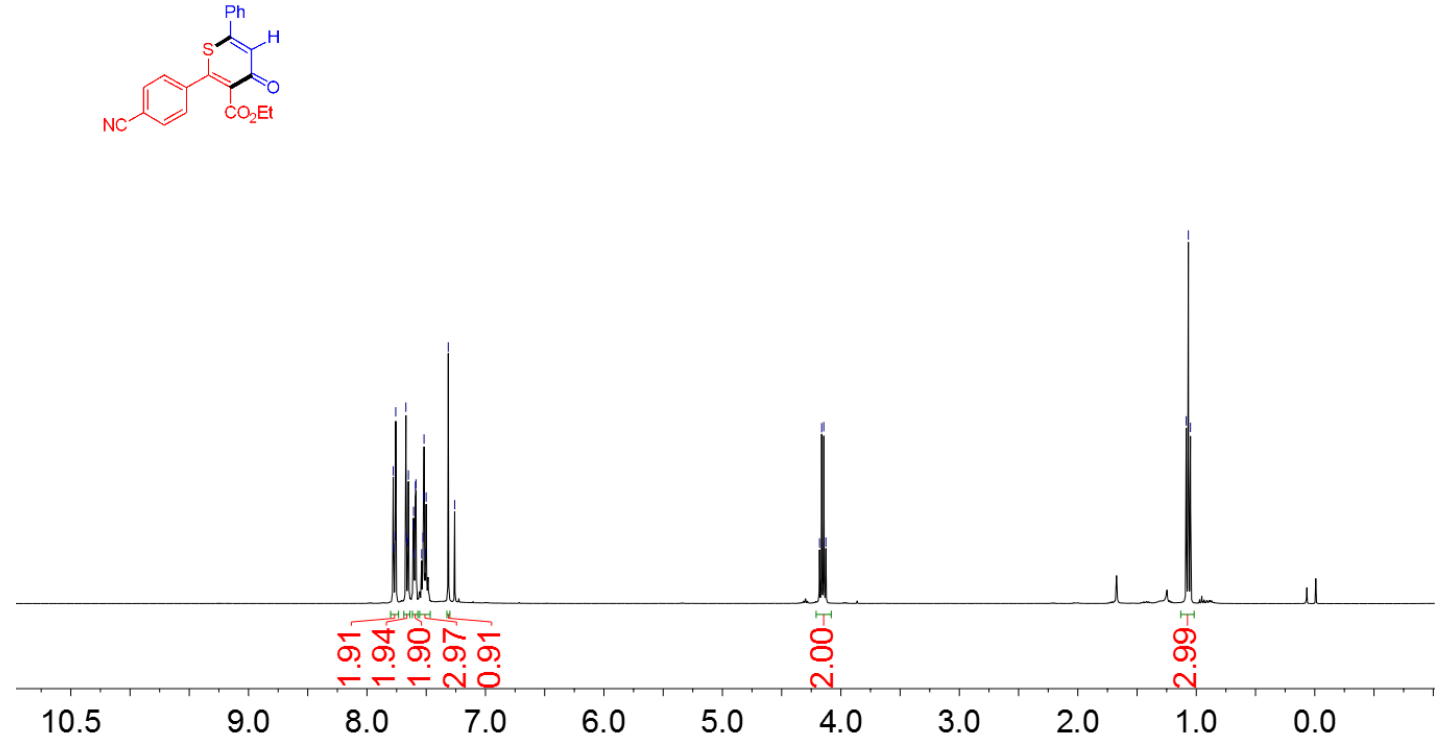

ON

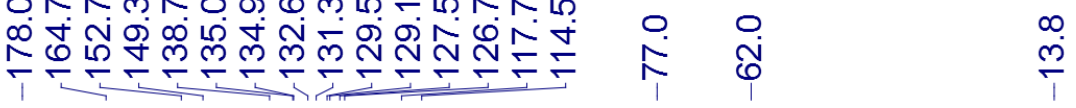
$\mathrm{CO}_{\mathrm{Et}}^{\mathrm{H}}$

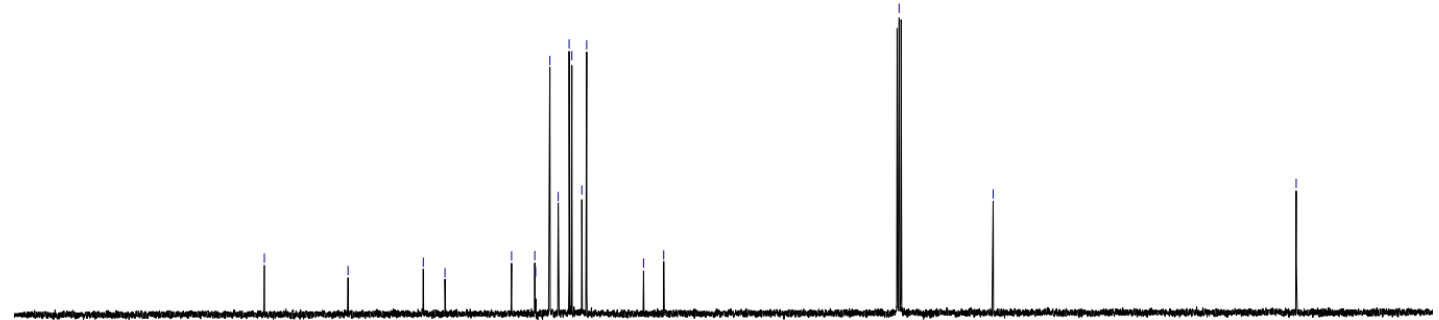

200 180 160 140 120 100 80

60

40 
${ }^{1} \mathrm{H}$ and ${ }^{13} \mathrm{C}$ of compound $4 \mathrm{la}$ in $\mathrm{CDCl}_{3}$

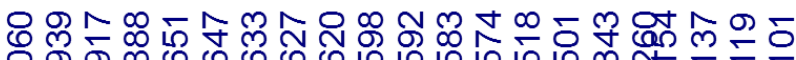

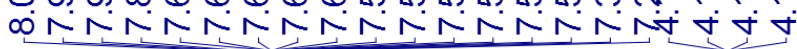

๖ำ

00
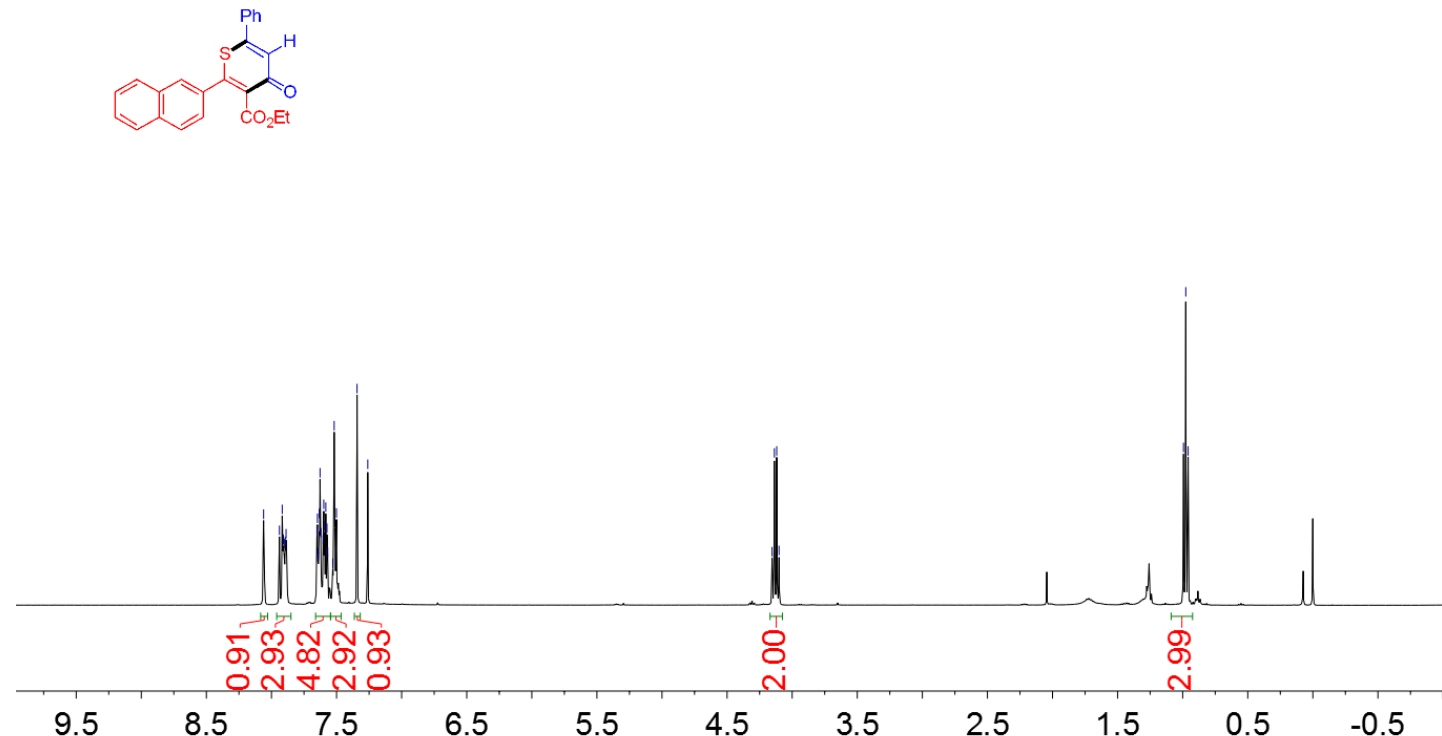

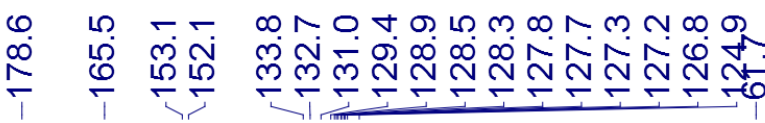
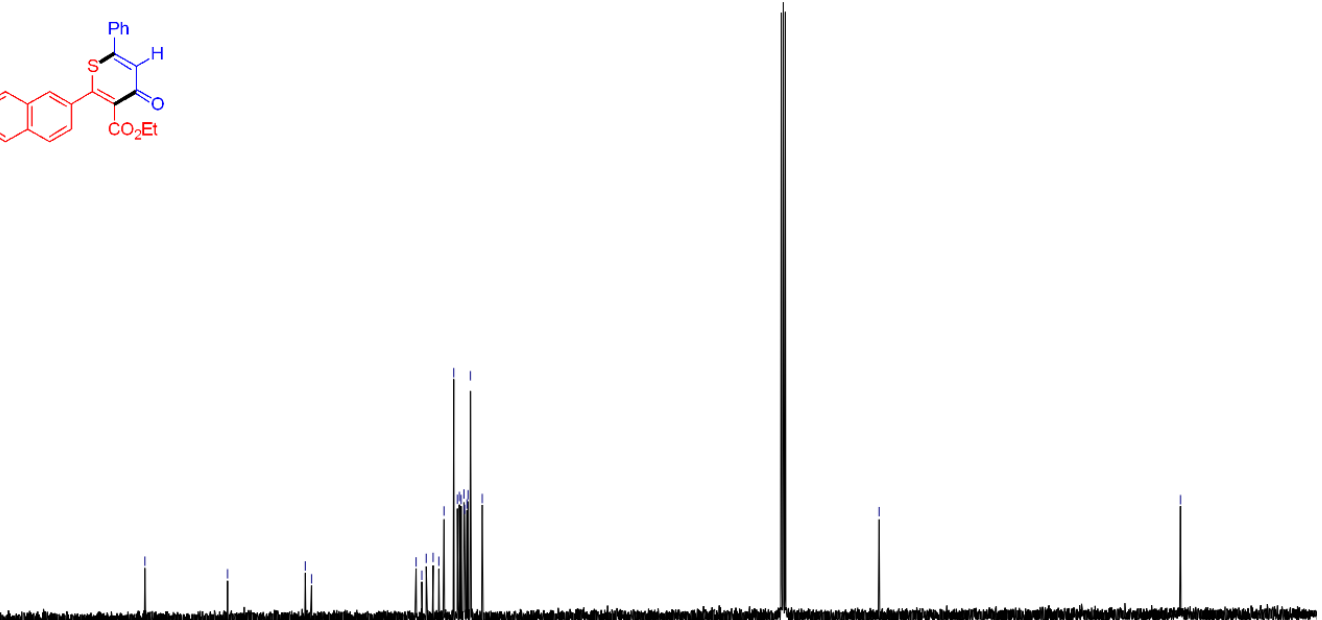

200

180

160

140

120

100

80

60

2 
${ }^{1} \mathrm{H}$ and ${ }^{13} \mathrm{C}$ of compound $4 \mathrm{ma}$ in $\mathrm{CDCl}_{3}$

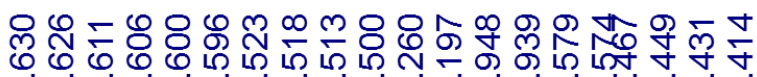

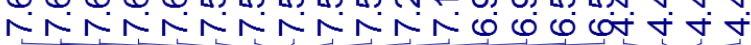

\& ํํㅇ
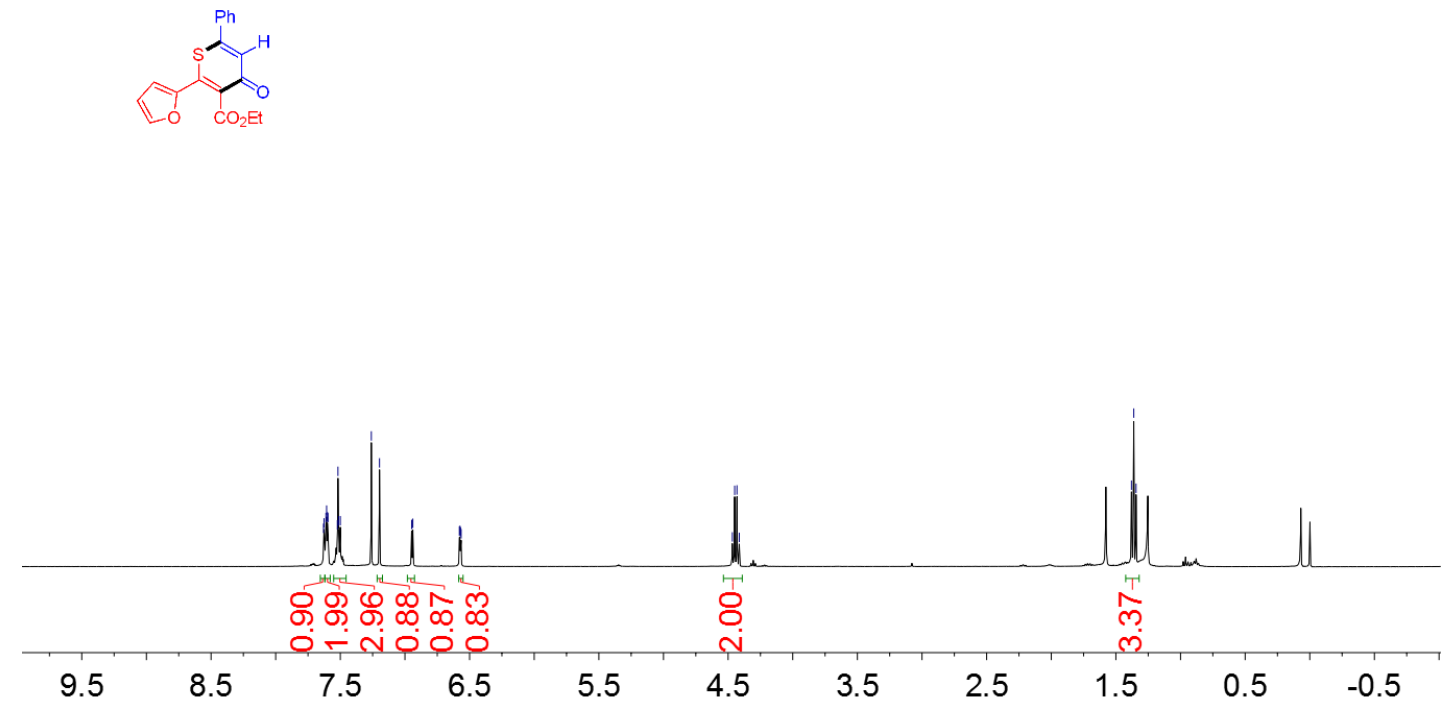

๑) Nِழூ
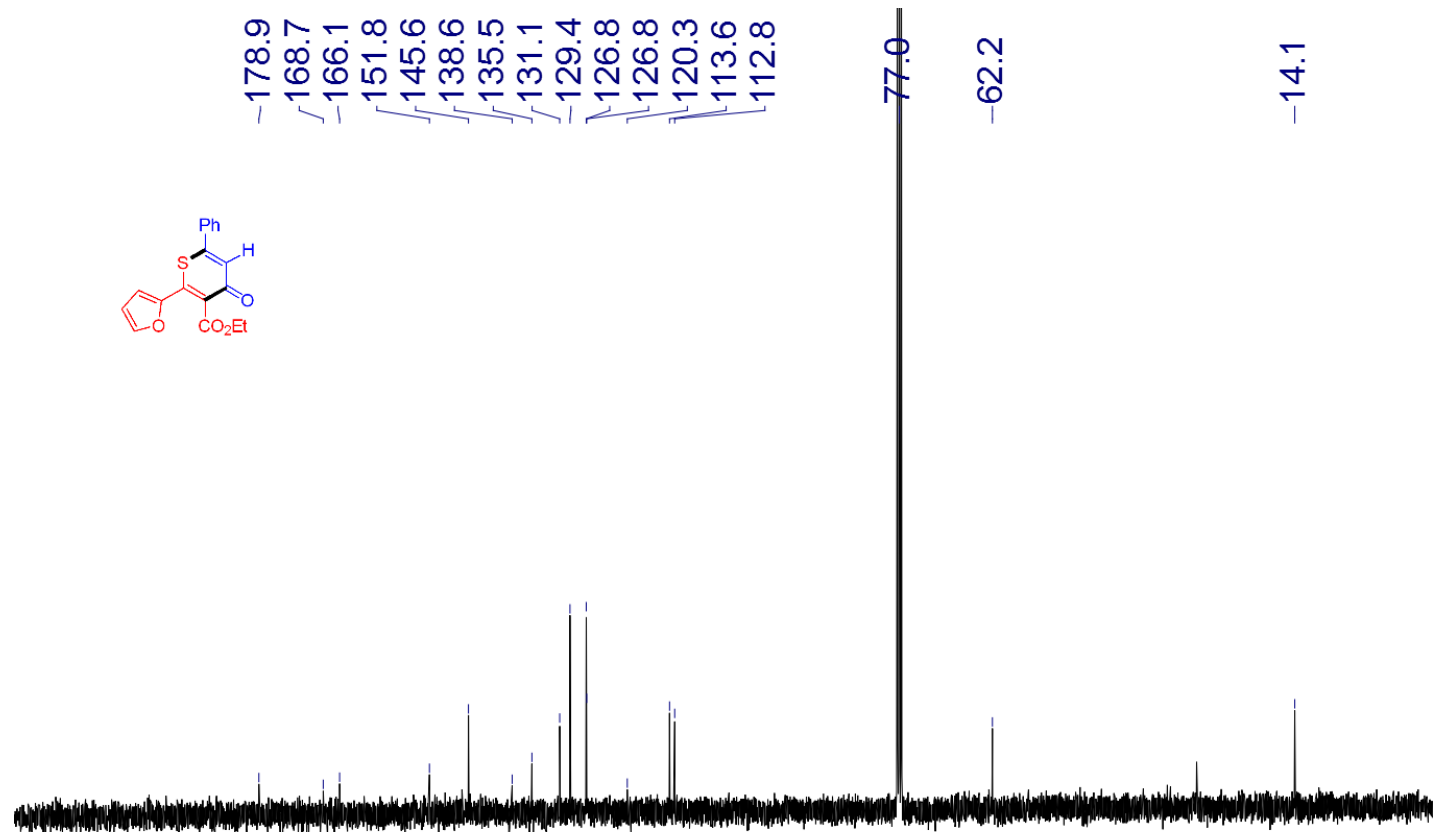

200 180 160 140 120 100

80

60

40

20 
${ }^{1} \mathrm{H}$ and ${ }^{13} \mathrm{C}$ of compound $4 \mathrm{na}$ in $\mathrm{CDCl}_{3}$

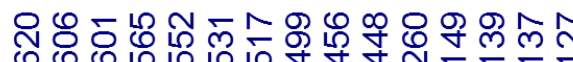

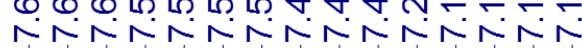
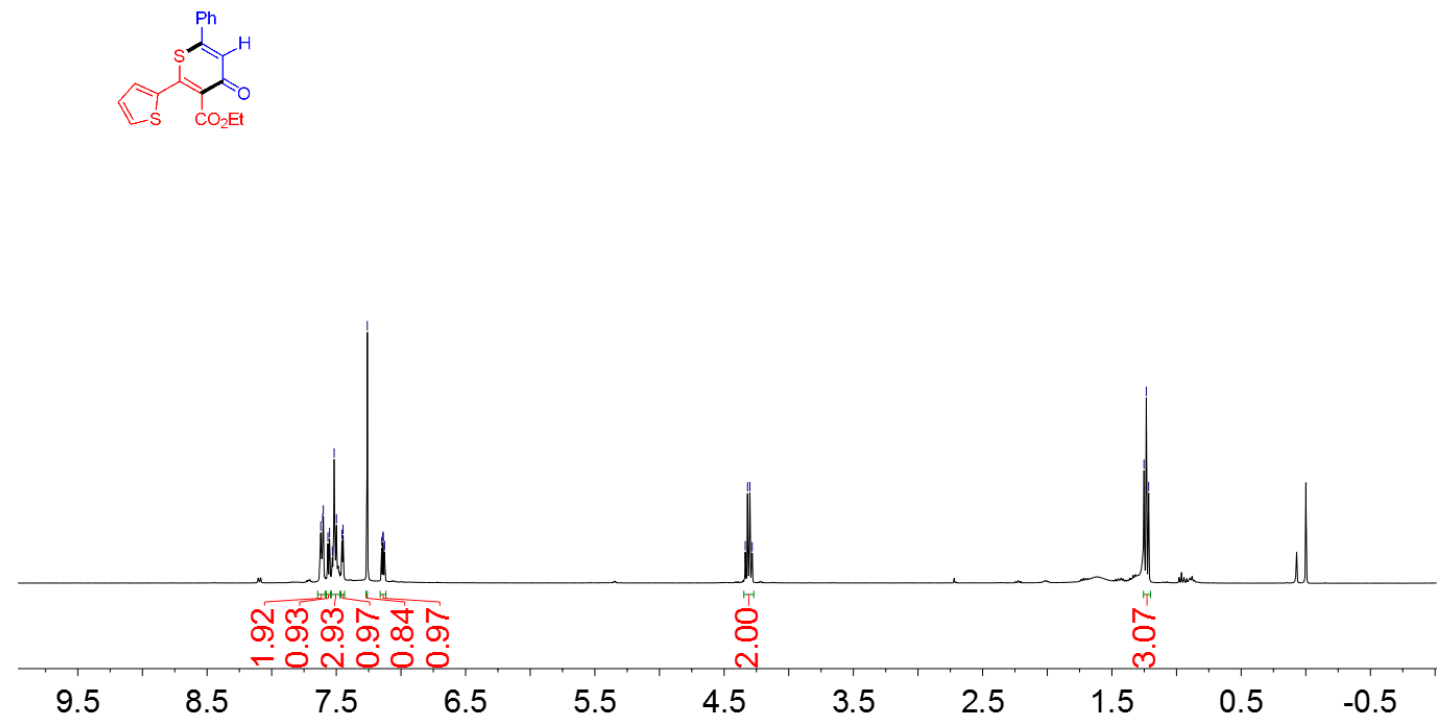

$\infty$ N ㄷํ

ชิ

ल)
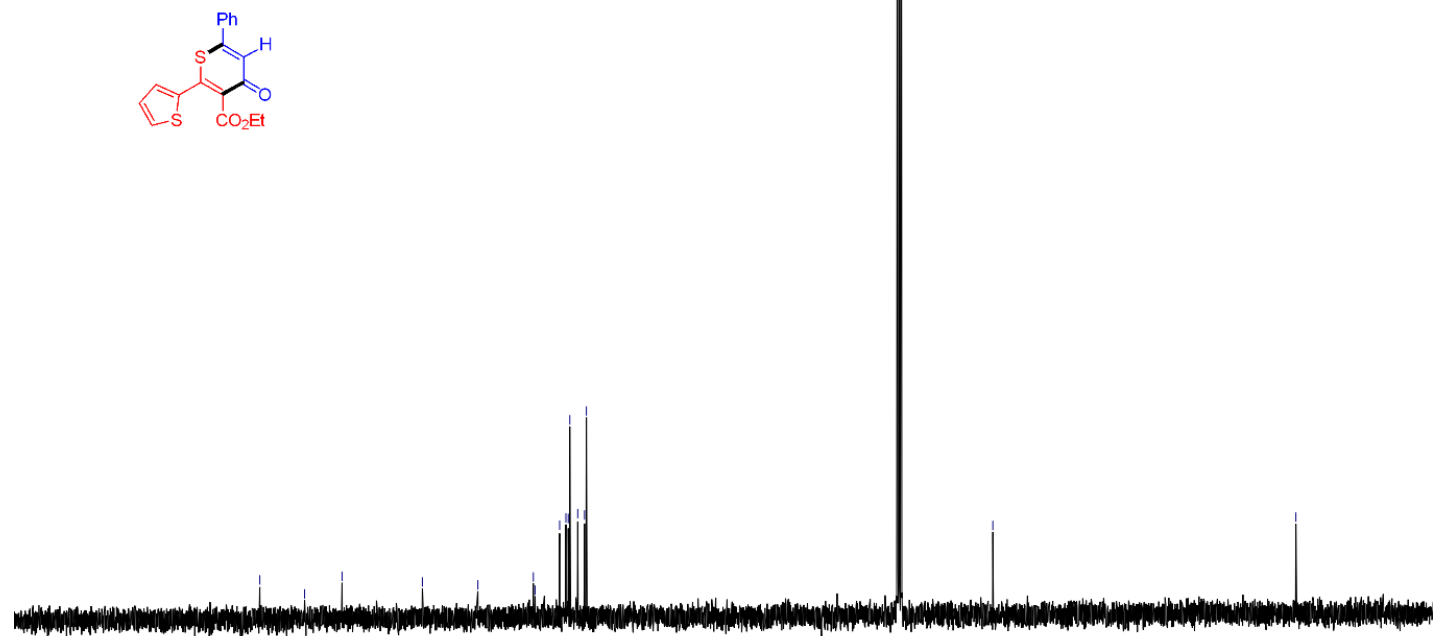

200

180

160

140

120

100

80

60

40

20 
${ }^{1} \mathrm{H}$ and ${ }^{13} \mathrm{C}$ of compound $40 a$ in $\mathrm{CDCl}_{3}$

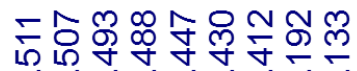

NNNNNNN

$\begin{array}{ll}\infty & 0 \\ \infty & N \\ \infty & n\end{array}$

8̊
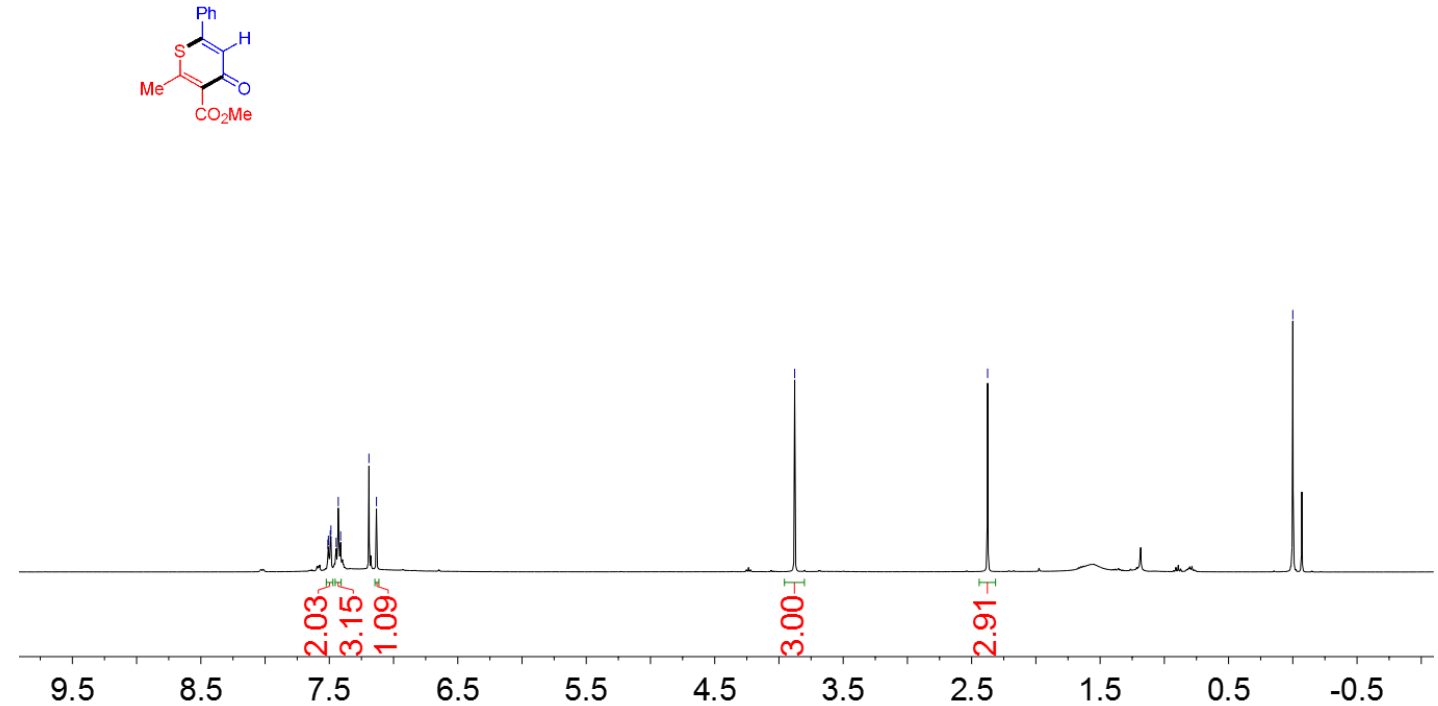

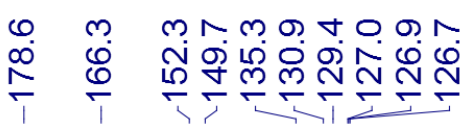
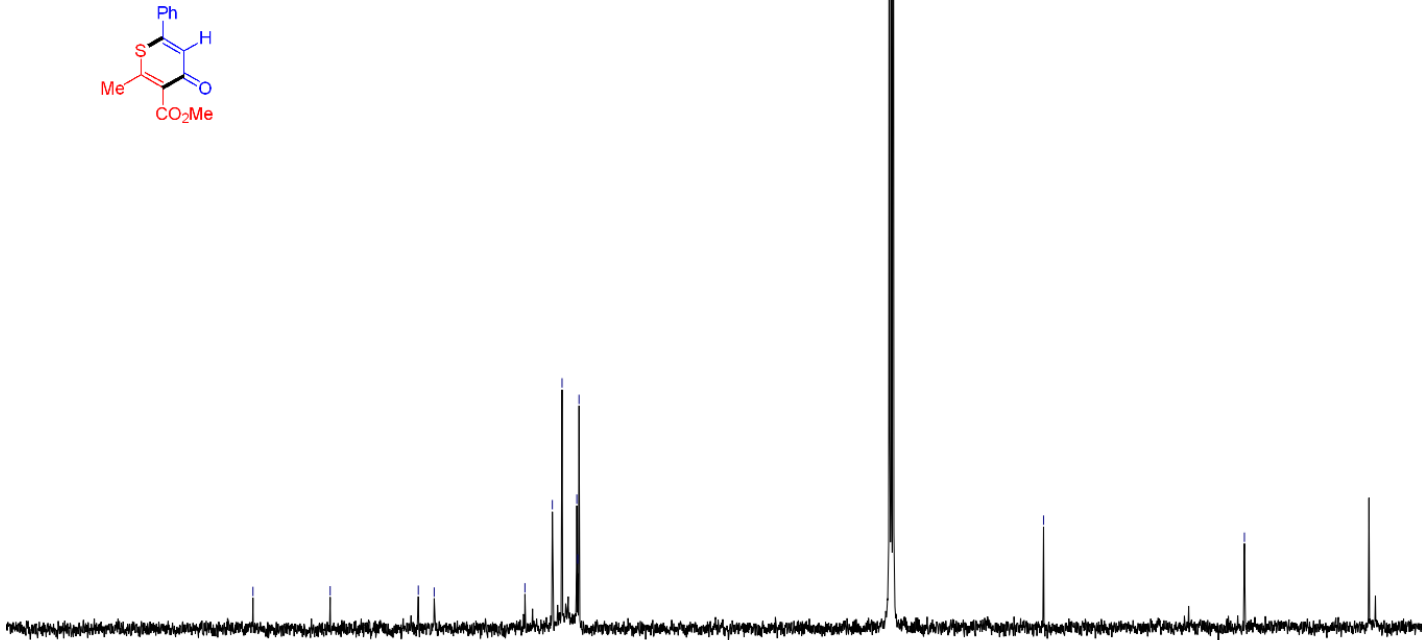

200

180

160

140

120

100

80

60

40

20

$\stackrel{\infty}{\infty}$ 
${ }^{1} \mathrm{H}$ and ${ }^{13} \mathrm{C}$ of compound $4 \mathrm{pa}$ in $\mathrm{CDCl}_{3}$

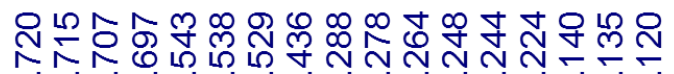

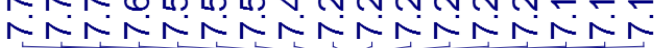
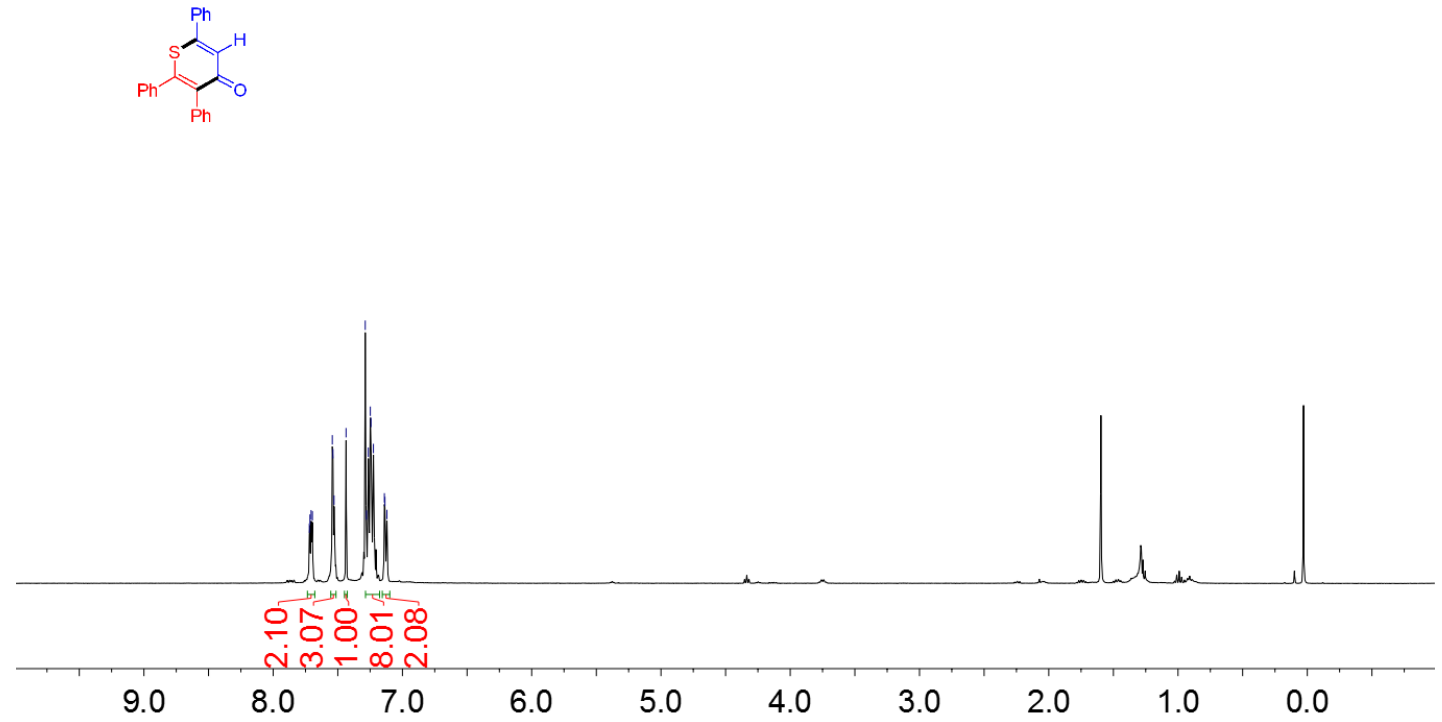

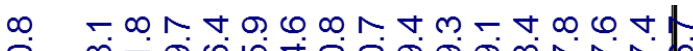

@
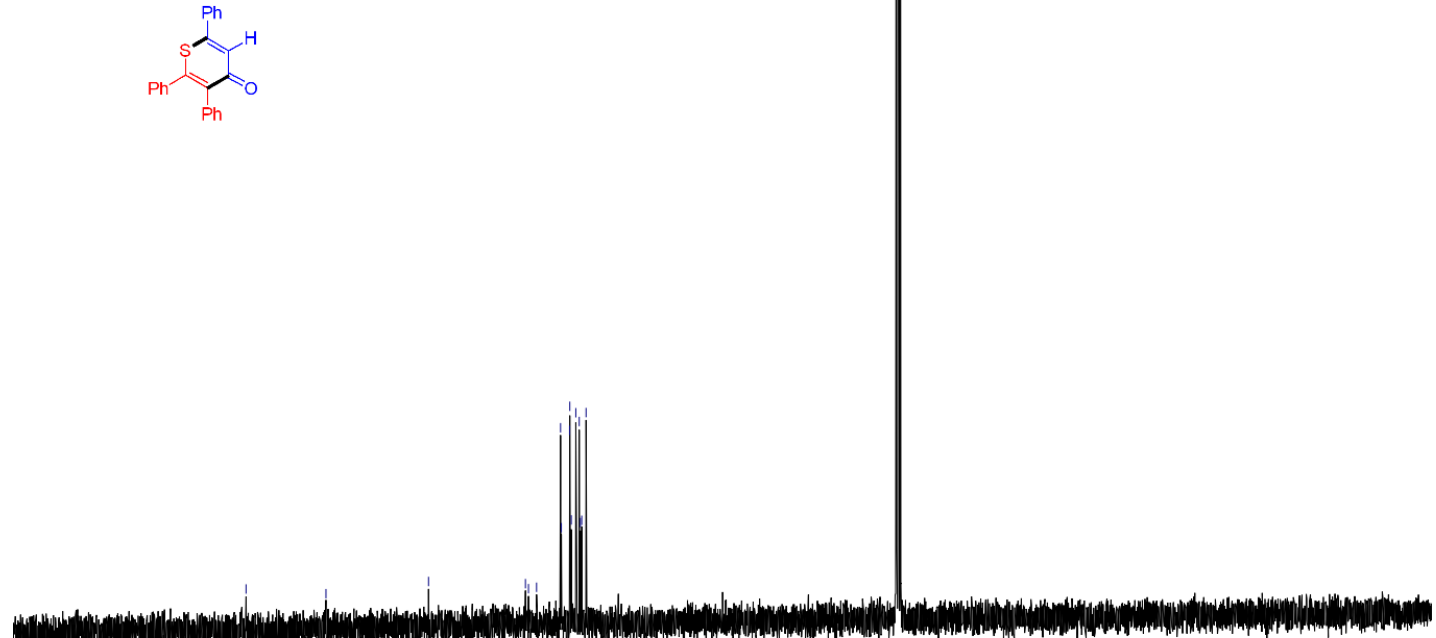

200

180

160

140

120

100

80

60

20 
${ }^{1} \mathrm{H}$ and ${ }^{13} \mathrm{C}$ of compound $4 \mathrm{qu}$ in $\mathrm{CDCl}_{3}$

๒
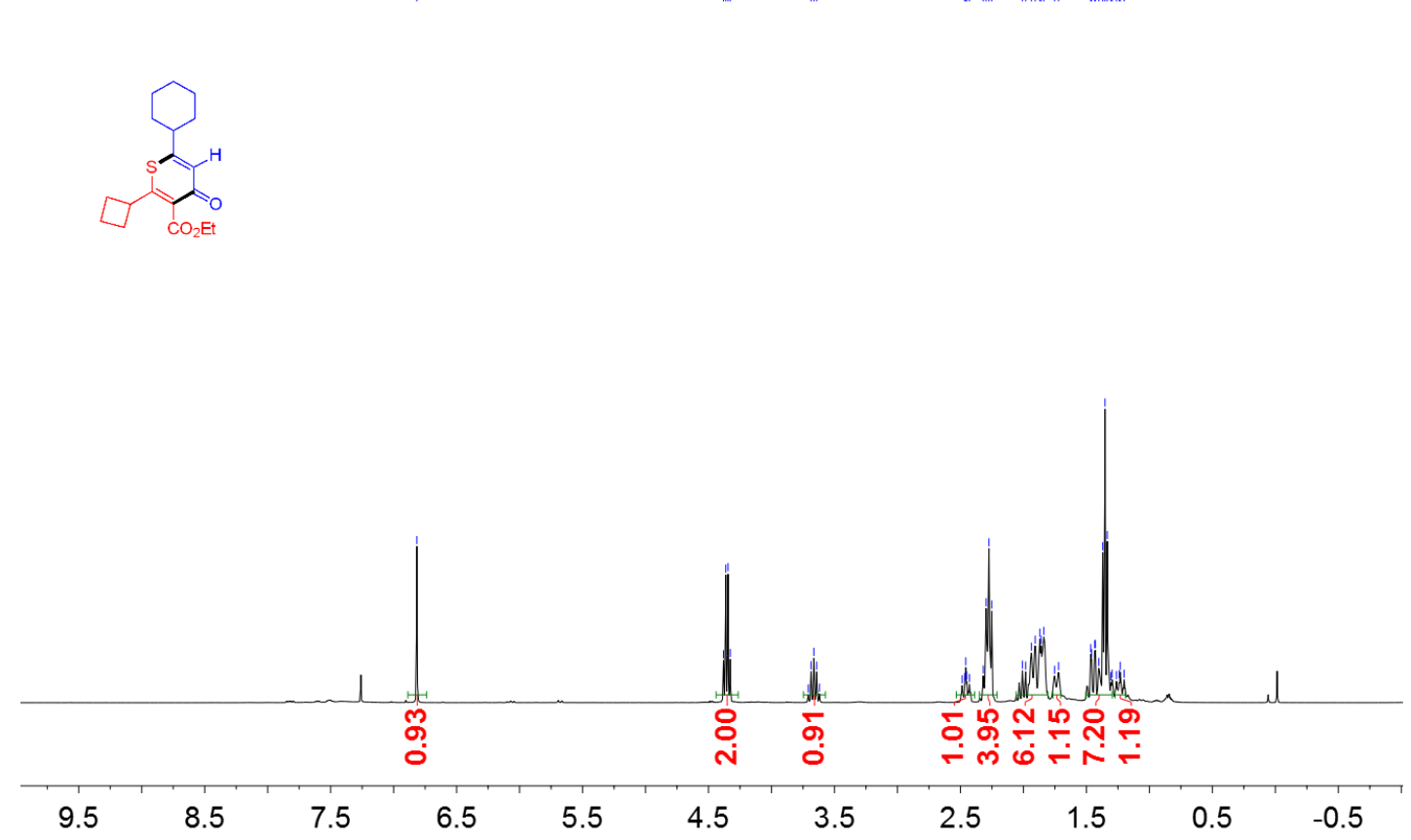

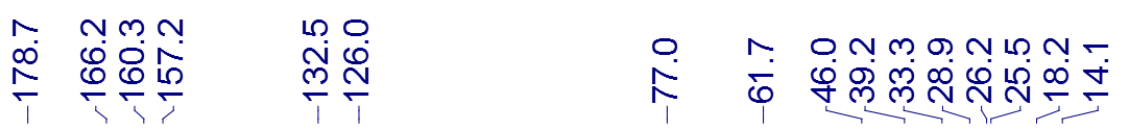
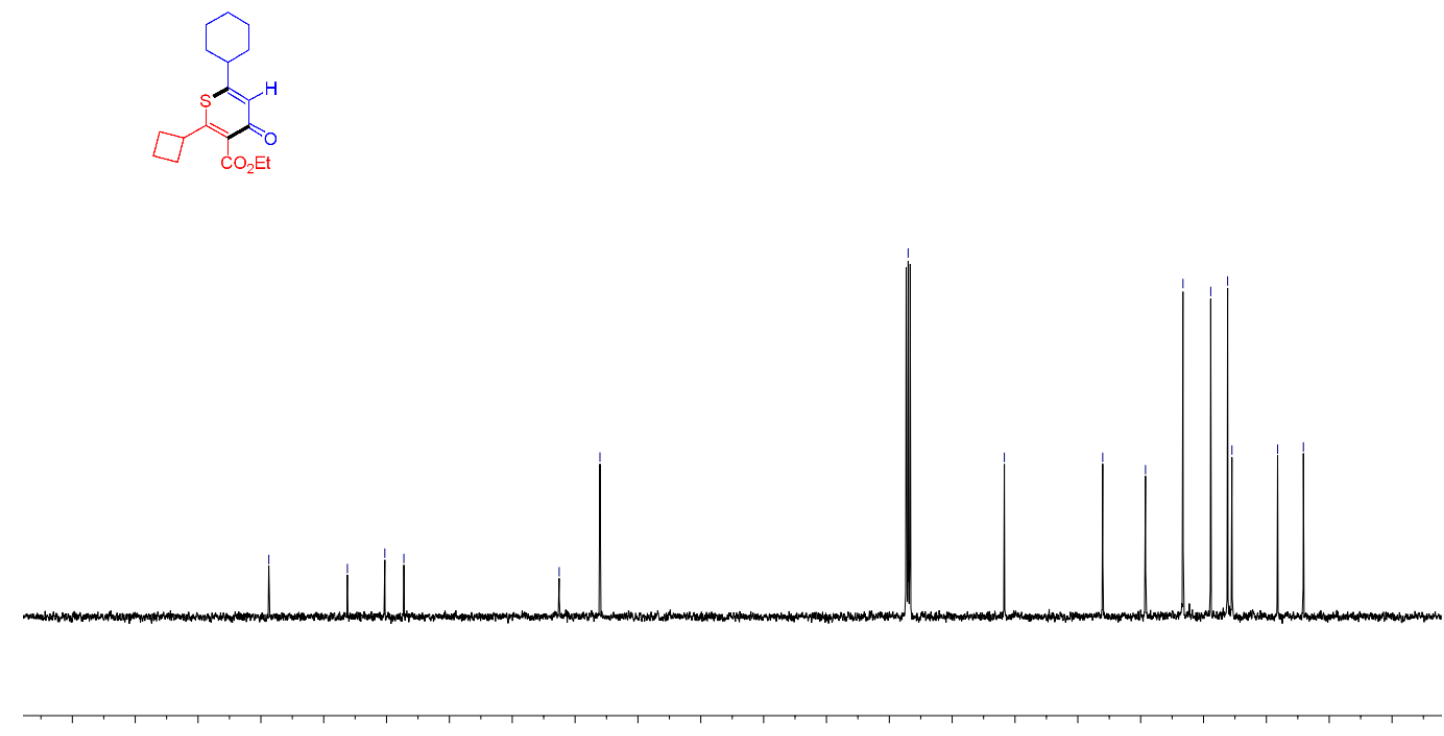

160

140

120

100

80

60

40

20 
${ }^{1} \mathrm{H}$ and ${ }^{13} \mathrm{C}$ of compound $4 \mathrm{ru}$ in $\mathrm{CDCl}_{3}$

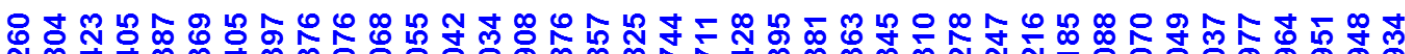

N
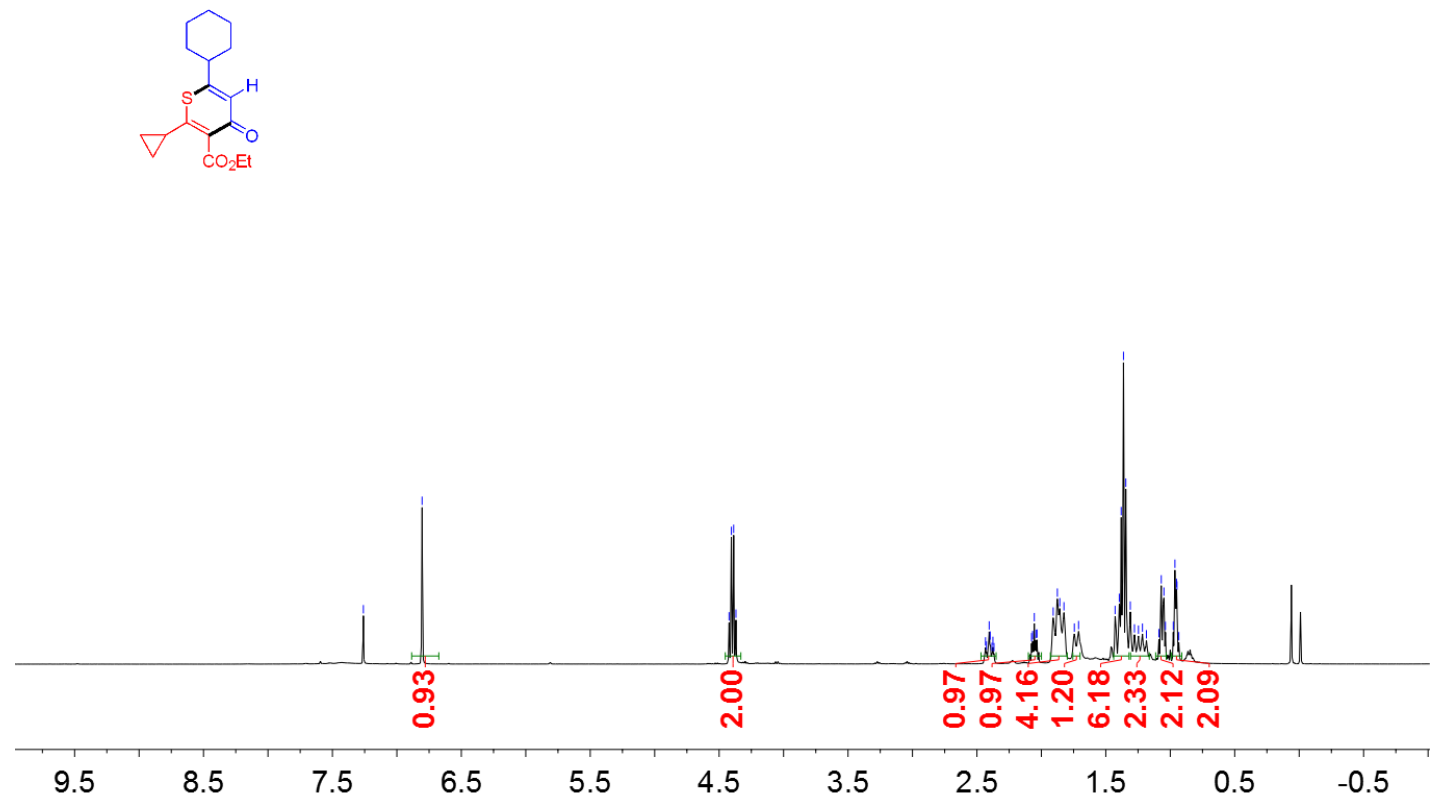

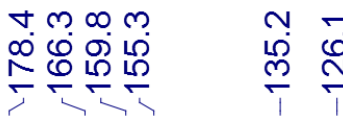

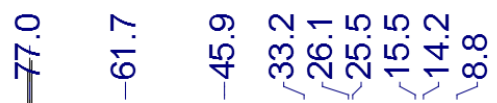
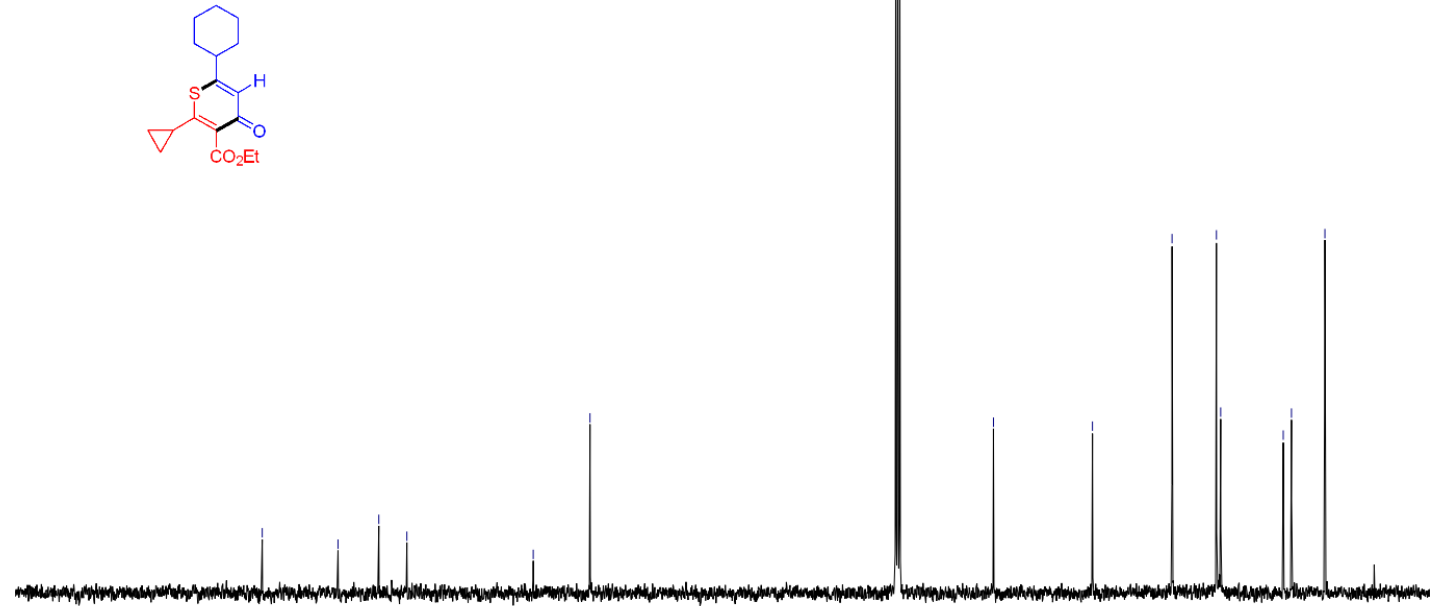

200

180

160

140

120

100

80

60

40

20 
${ }^{1} \mathrm{H}$ and ${ }^{13} \mathrm{C}$ of compound 13 in $\mathrm{CDCl}_{3}$

以

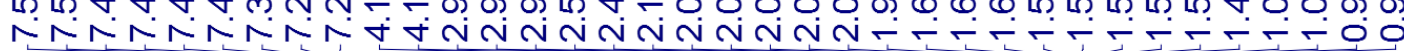
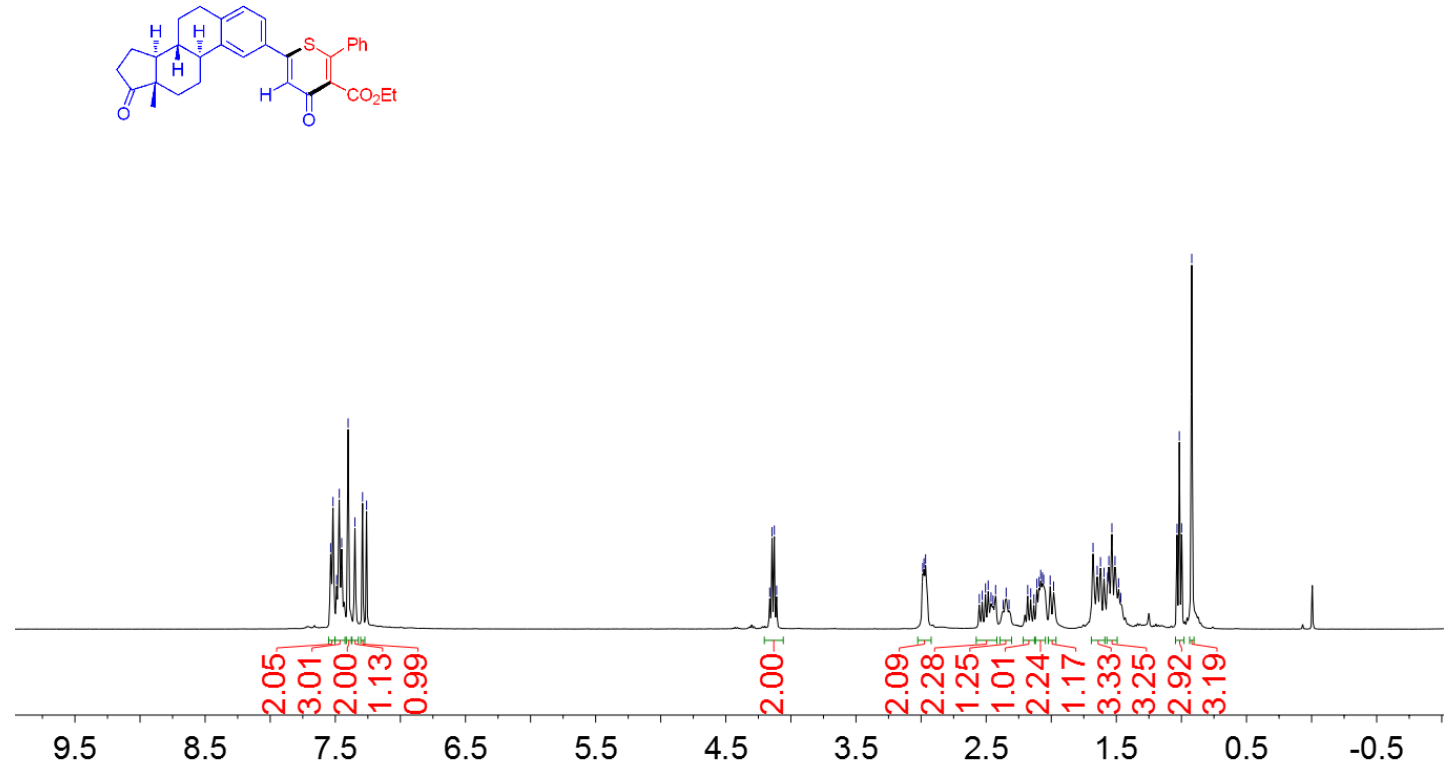

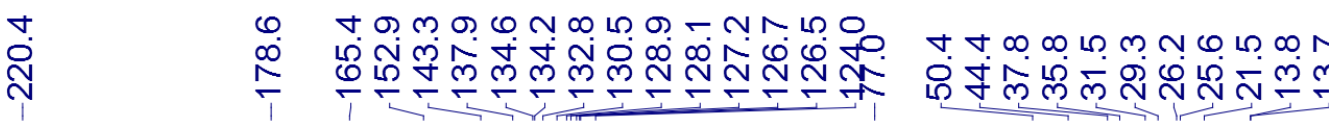
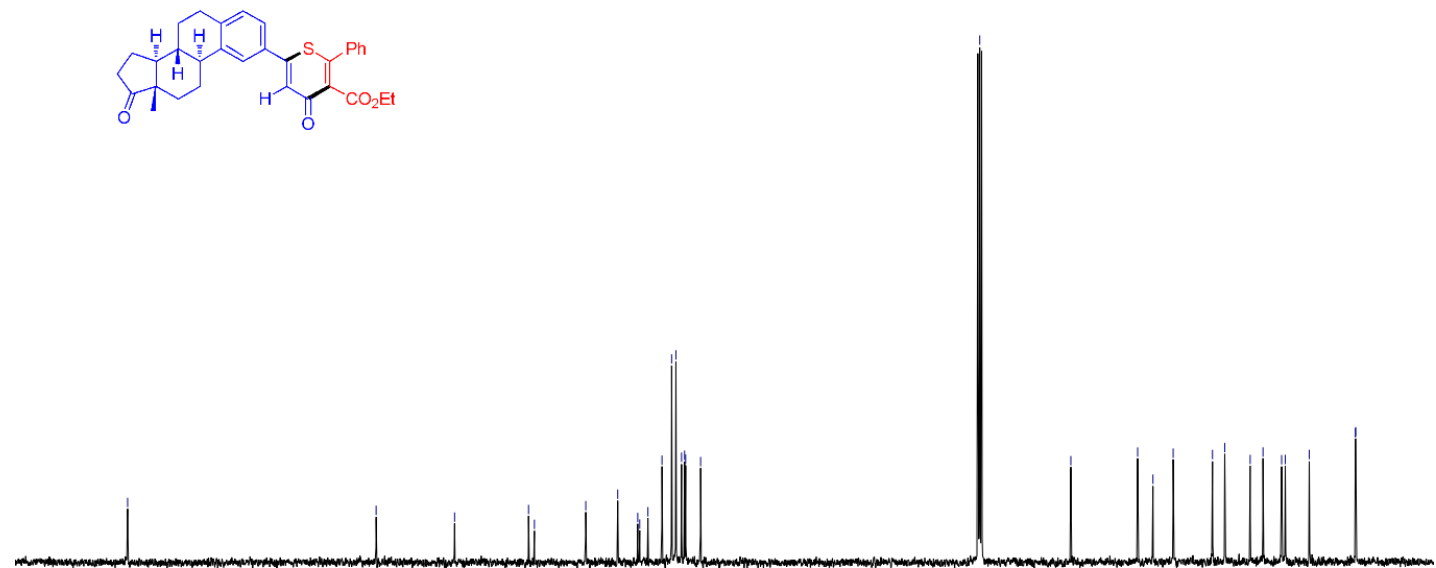
${ }^{1} \mathrm{H}$ and ${ }^{13} \mathrm{C}$ of compound 16 in $\mathrm{CDCl}_{3}$

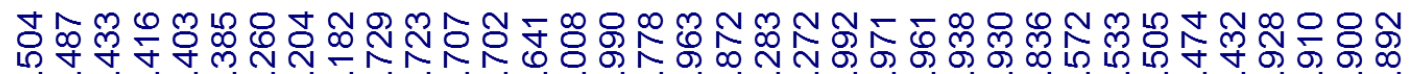

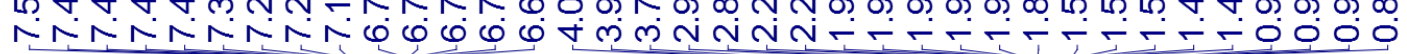

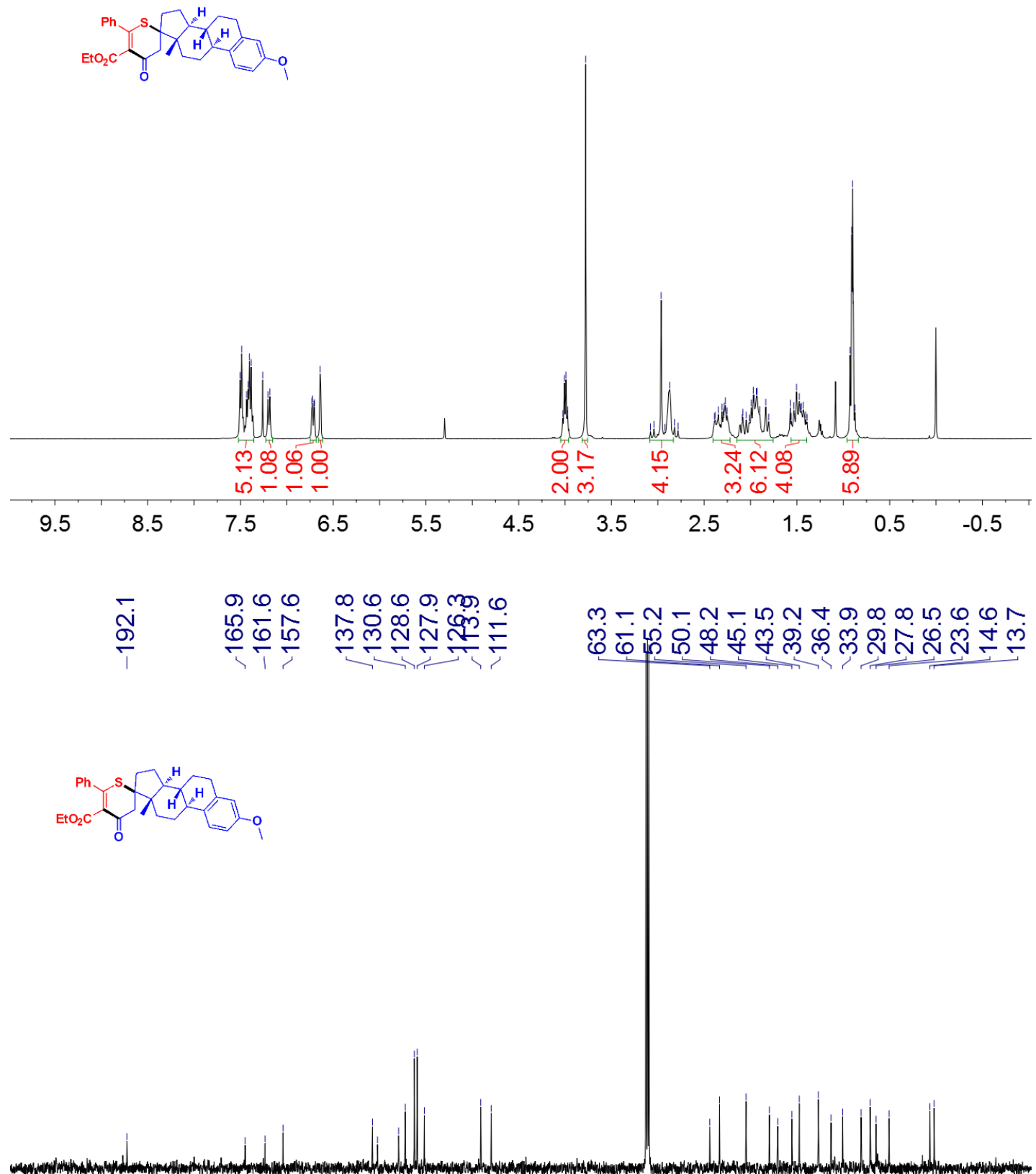

200 180 160 140 120 100

80

60

40

20

0


${ }^{1} \mathrm{H}$ and ${ }^{13} \mathrm{C}$ of compound $\mathrm{O}-4 \mathrm{aa}$ in $\mathrm{CDCl}_{3}$

Г্

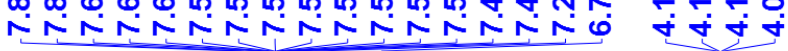

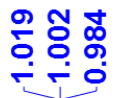
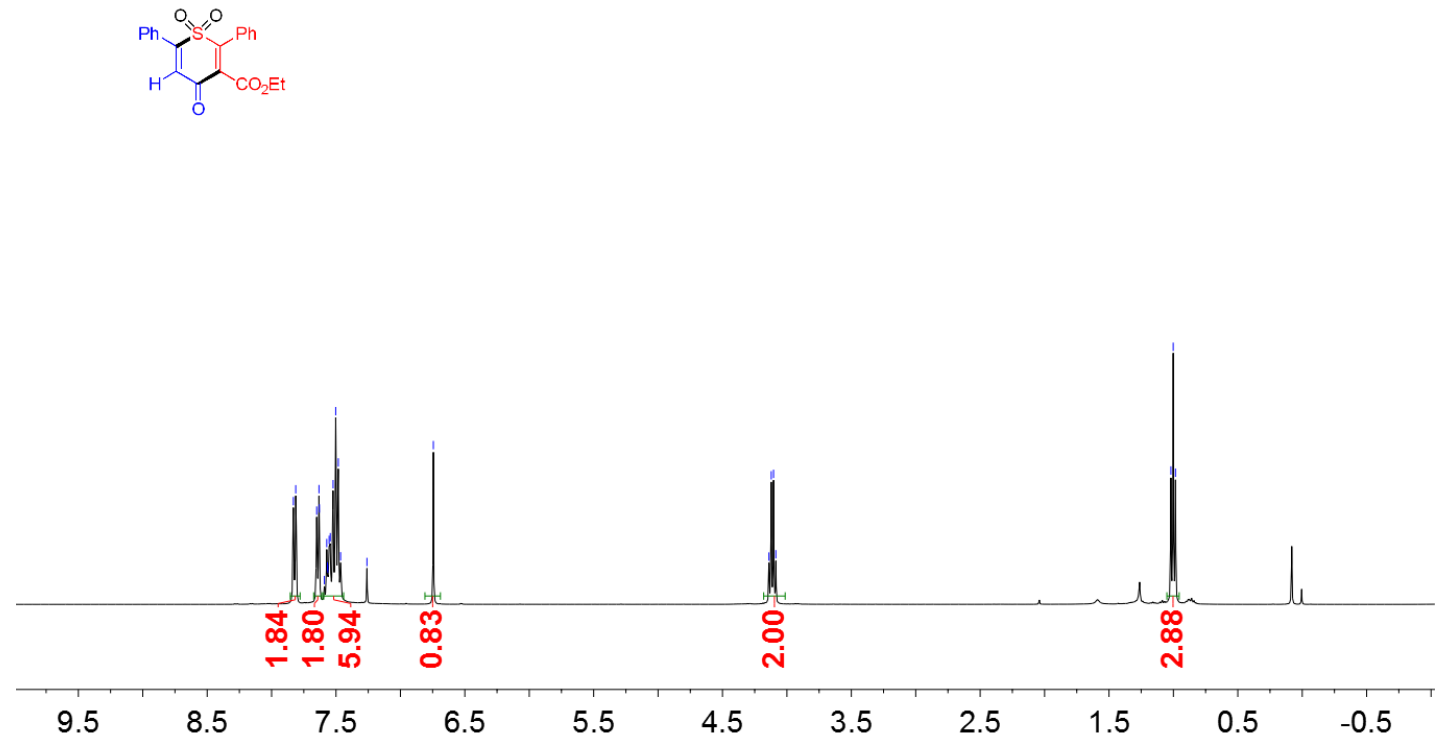

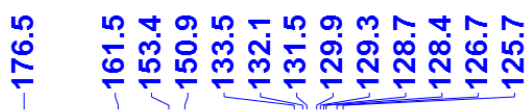
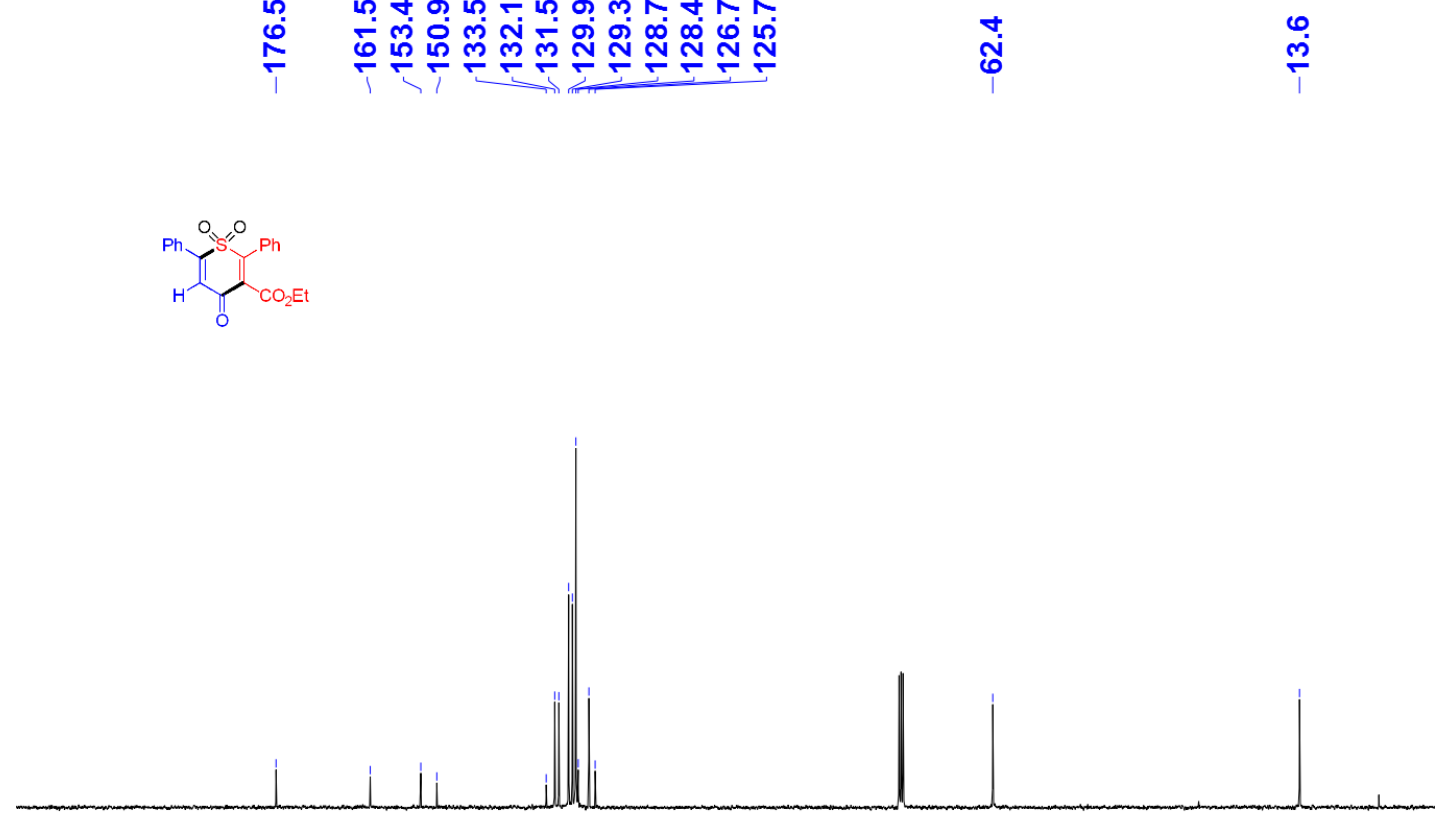

200

180

160

140

120

100

80

60

40

20 
${ }^{1} \mathrm{H}$ and ${ }^{13} \mathrm{C}$ of compound $4 \mathrm{aa}$ and $\boldsymbol{d}$-4aa in $\mathrm{CDCl}_{3}$

\begin{tabular}{|c|c|c|}
\hline 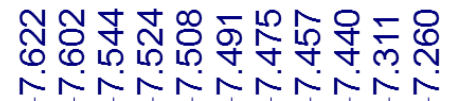 & 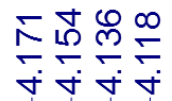 & 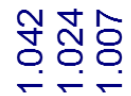 \\
\hline
\end{tabular}

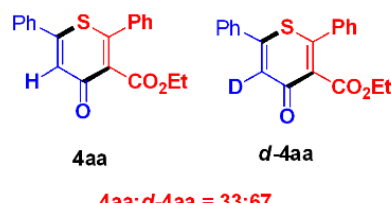

$4 \mathrm{aa}: d-4 \mathrm{aa}=33: 67$
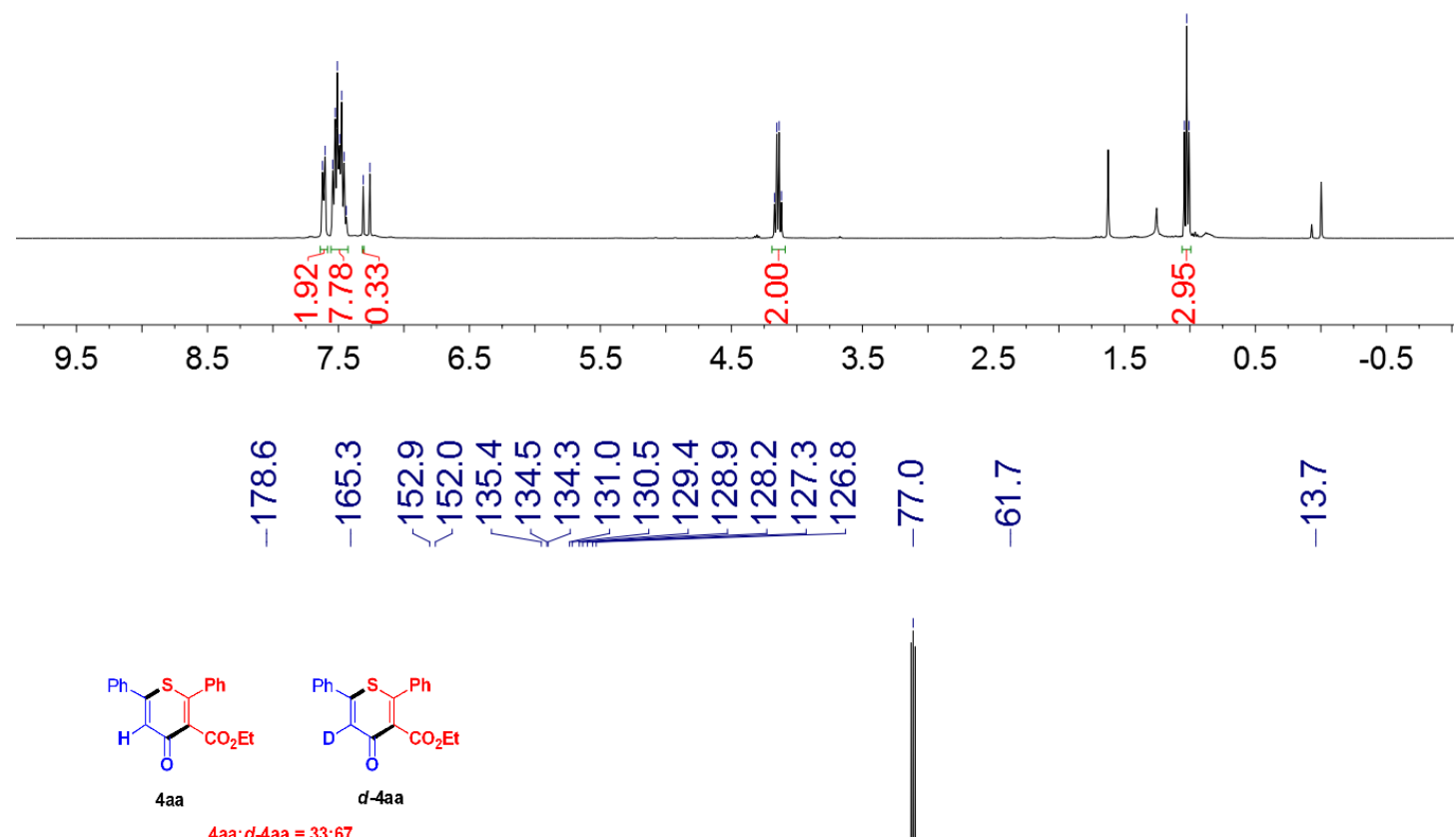

$: d-4 a=33: 67$

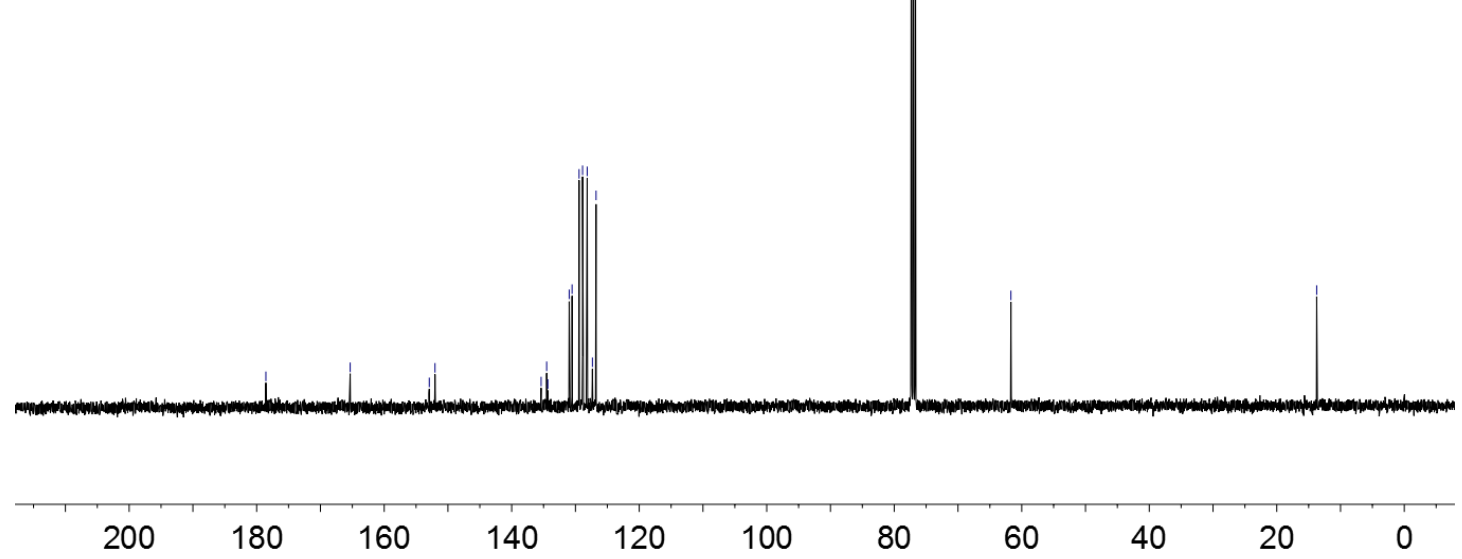

Copies of NMR Spectra of Some Materials 
${ }^{1} \mathrm{H}$ and ${ }^{13} \mathrm{C}$ of compound $5 \mathrm{~g}$ in $\mathrm{CDCl}_{3}$

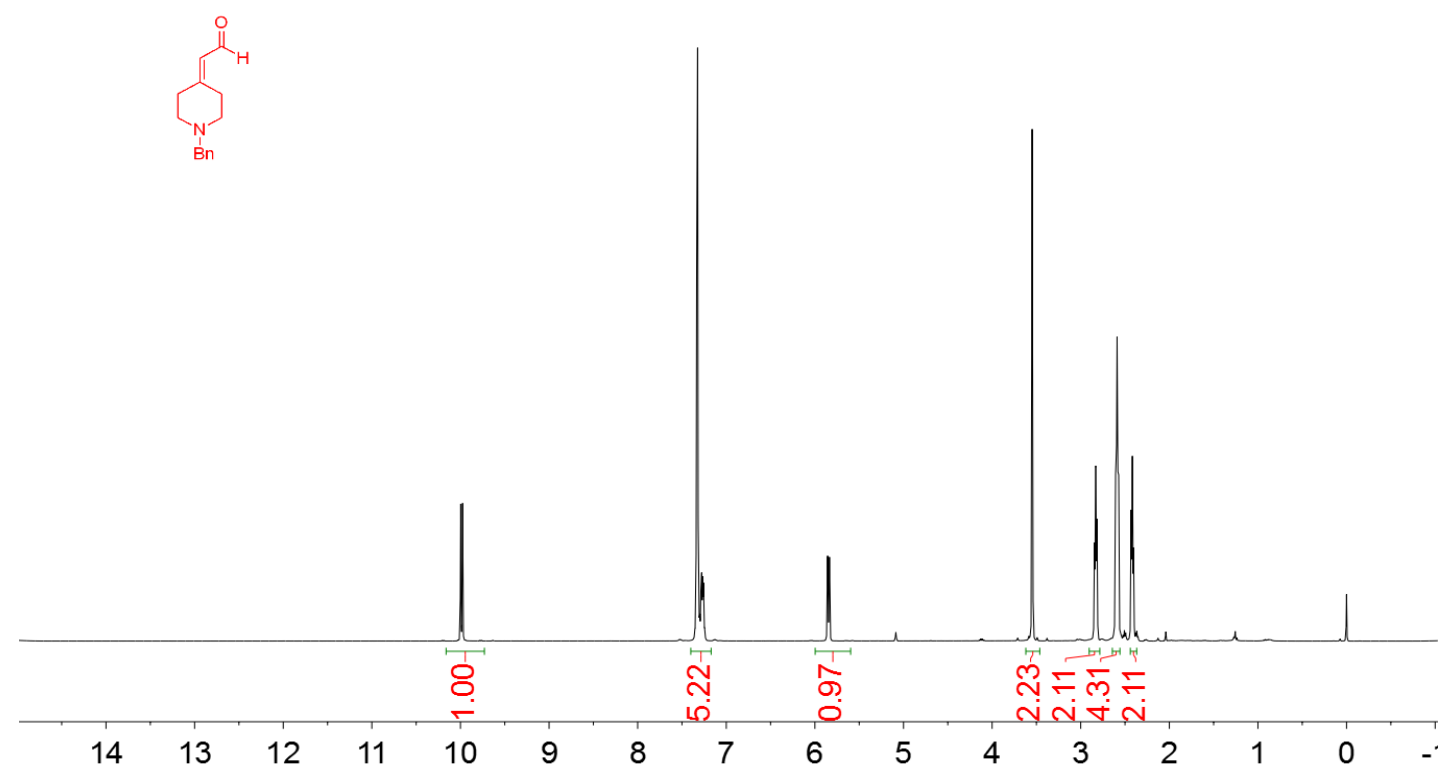

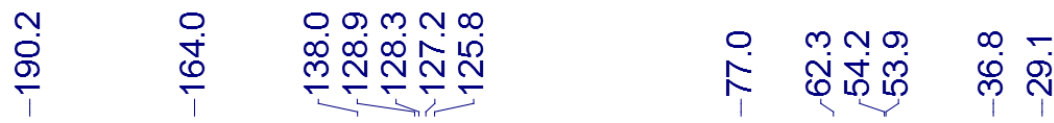
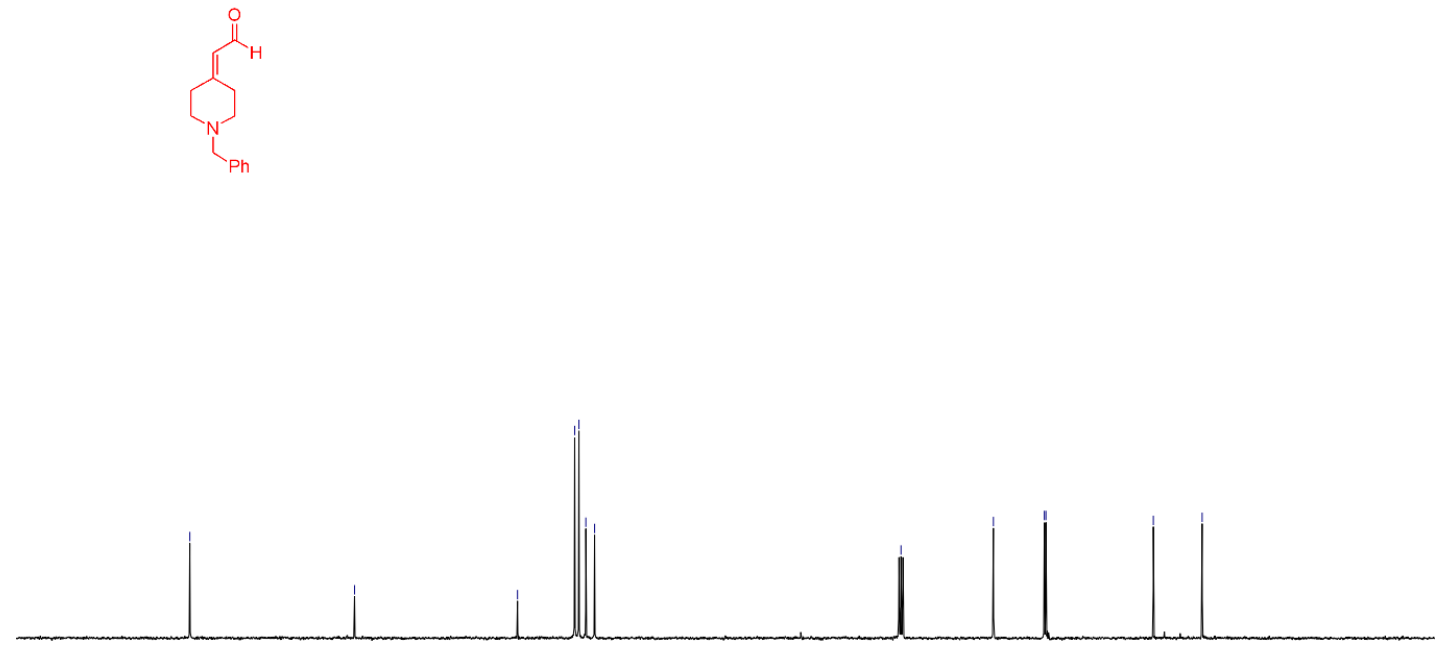

200 $180 \quad 160$ $140 \quad 120$ 100 80 60 $40 \quad 20$ 0 
${ }^{1} \mathrm{H}$ and ${ }^{13} \mathrm{C}$ of compound $5 \mathrm{j}$ in $\mathrm{CDCl}_{3}$

চ "

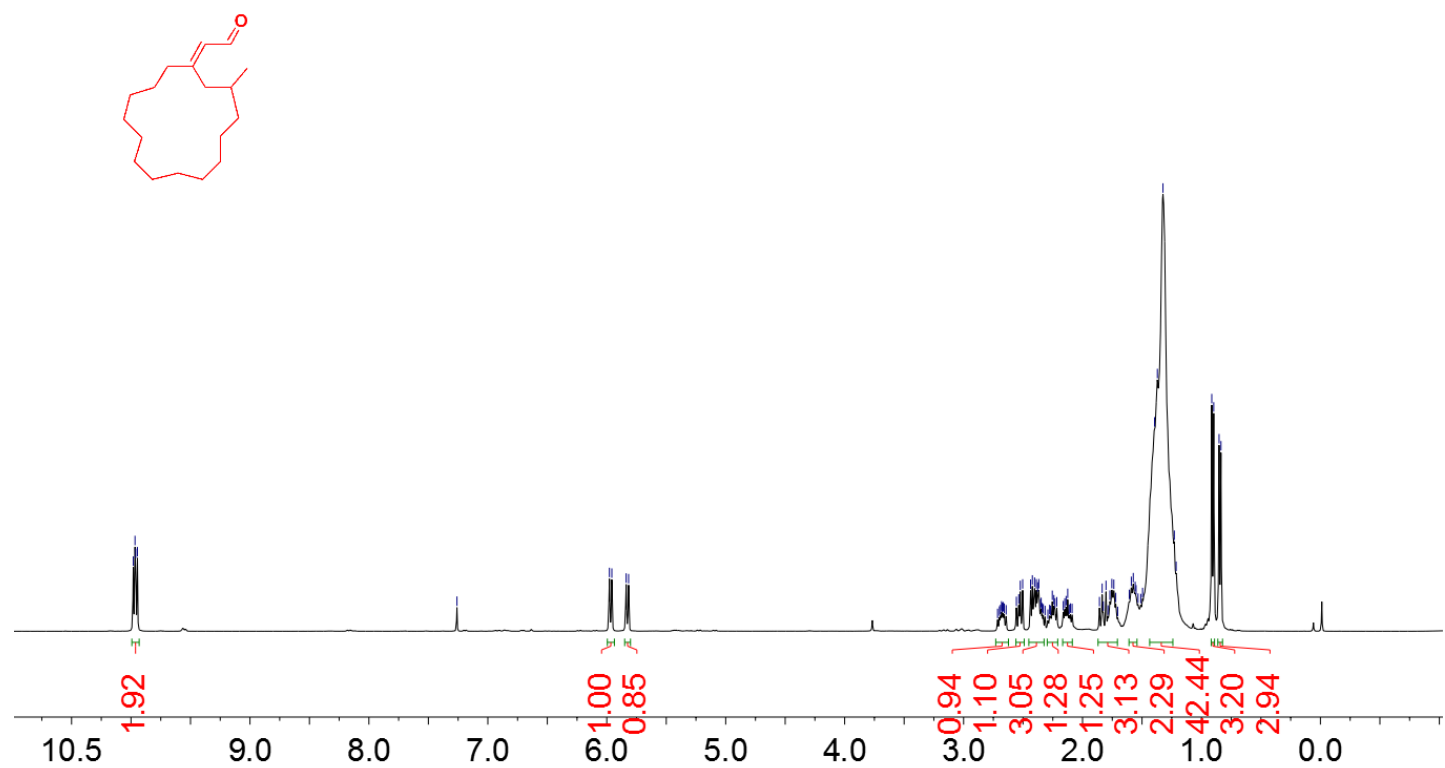

\begin{tabular}{|c|c|c|}
\hline 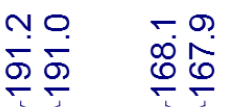 & $\begin{array}{l}\circ \stackrel{\circ}{\circ} \\
\stackrel{\text { Dे }}{N}\end{array}$ & 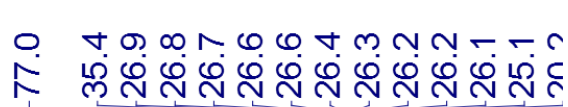 \\
\hline
\end{tabular}
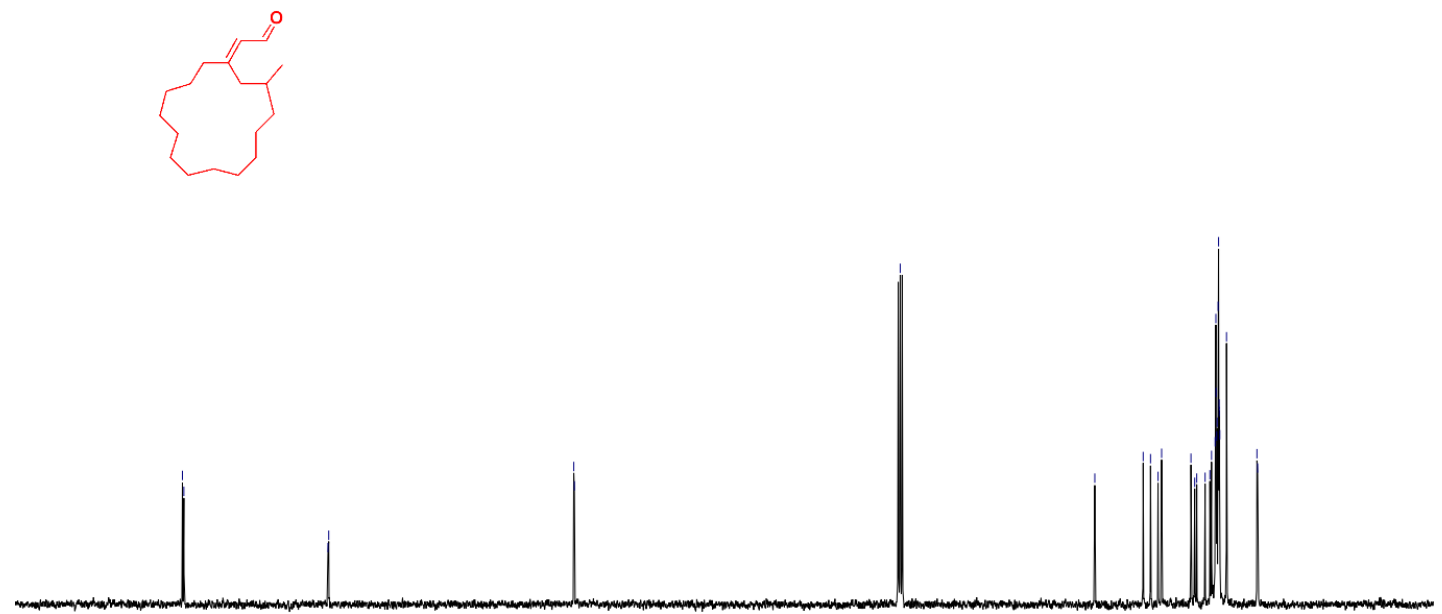

200

180

160

140

120

100

80

60

20 
${ }^{1} \mathrm{H}$ and ${ }^{13} \mathrm{C}$ of compound 10 in $\mathrm{CDCl}_{3}$

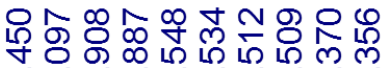

क⿻
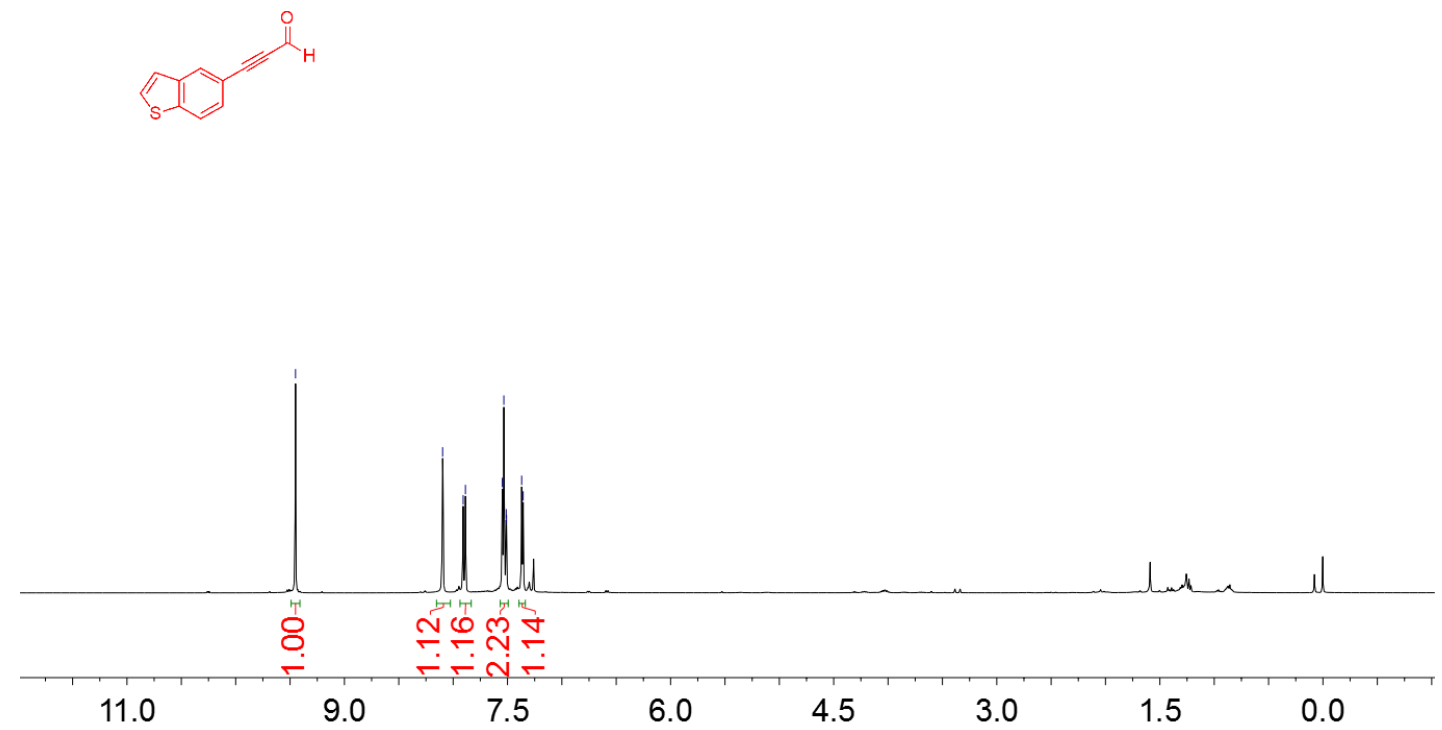

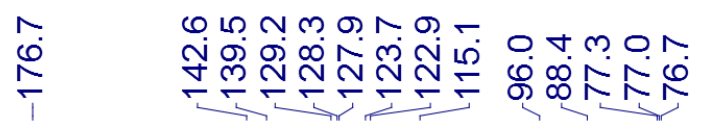

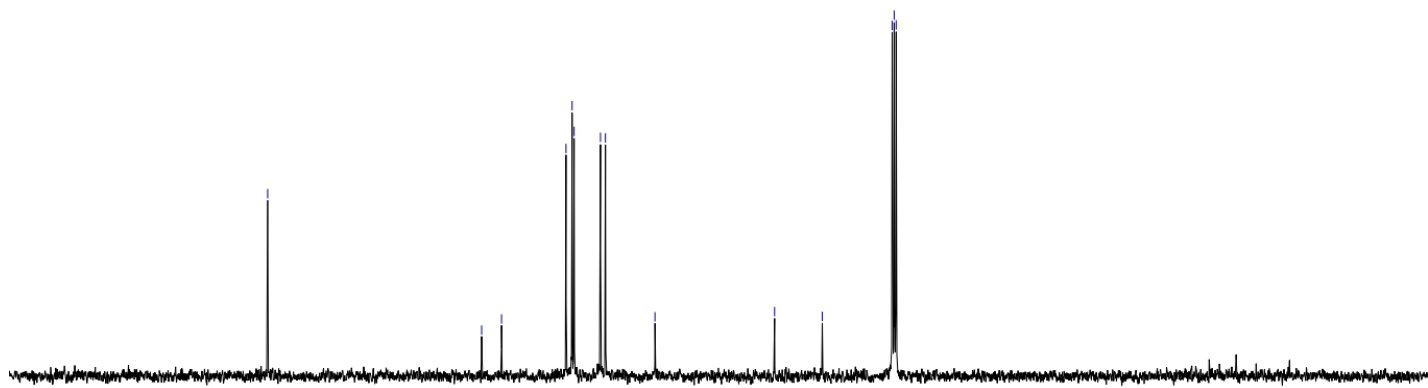

200

180

160

140

120

100

80

60

40

20 
${ }^{1} \mathrm{H}$ and ${ }^{13} \mathrm{C}$ of compound $1 \mathrm{~b}$ in $\mathrm{CDCl}_{3}$

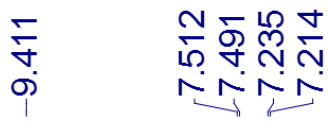

:
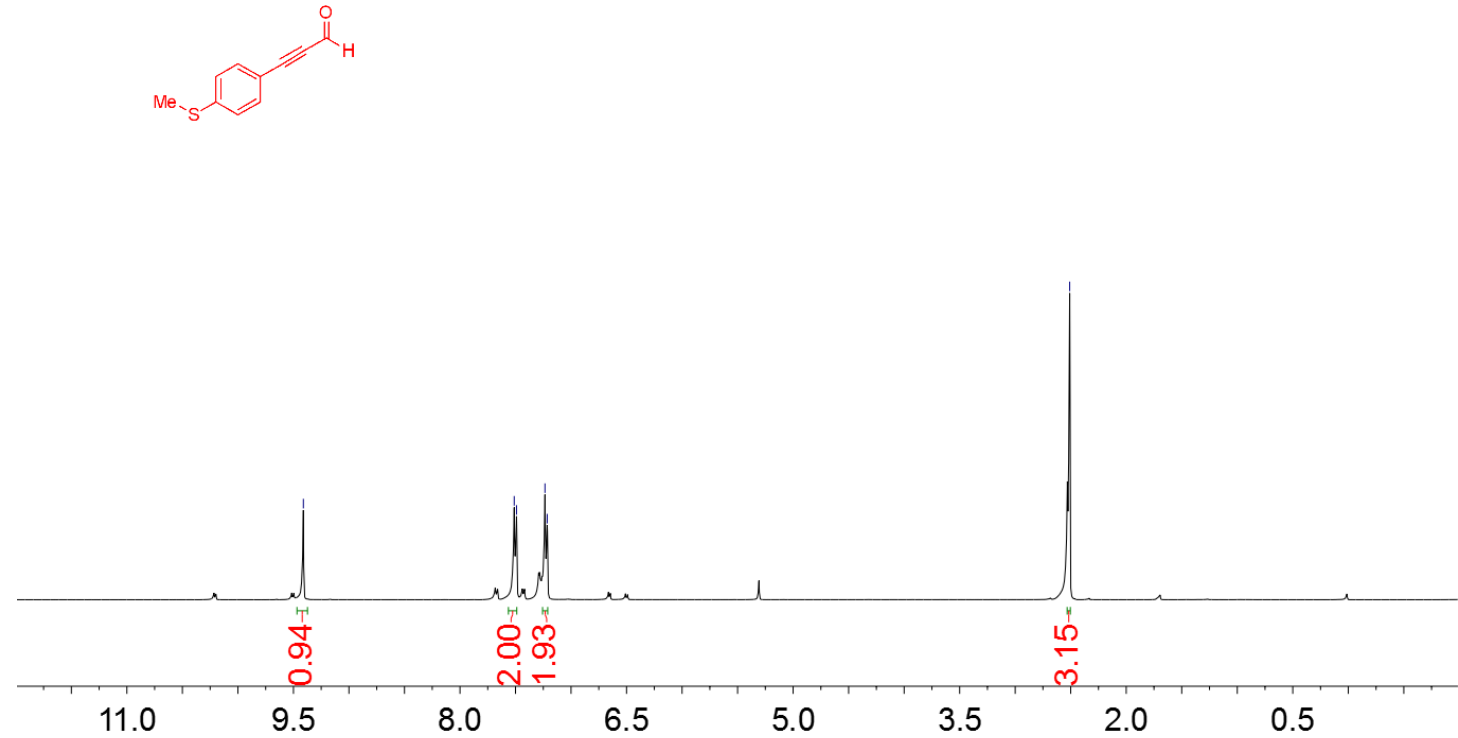

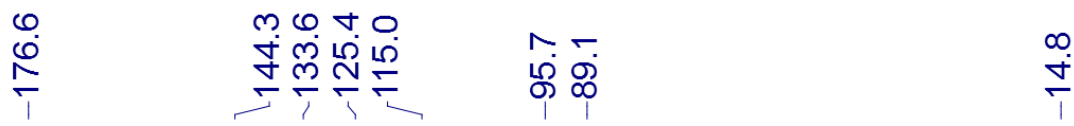

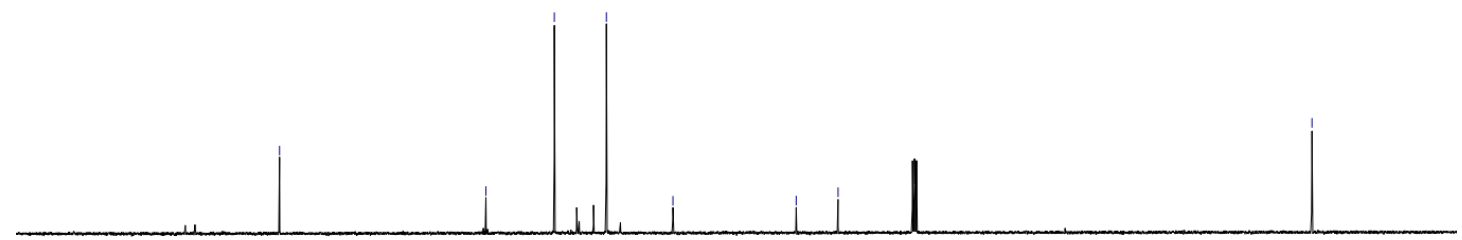

200

$180 \quad 160 \quad 140$

120

100

80

40

20

0

${ }^{1} \mathrm{H}$ and ${ }^{13} \mathrm{C}$ of compound 12 in $\mathrm{CDCl}_{3}$ 


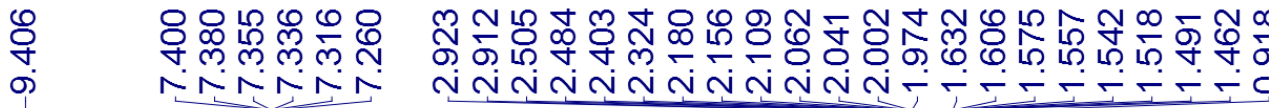

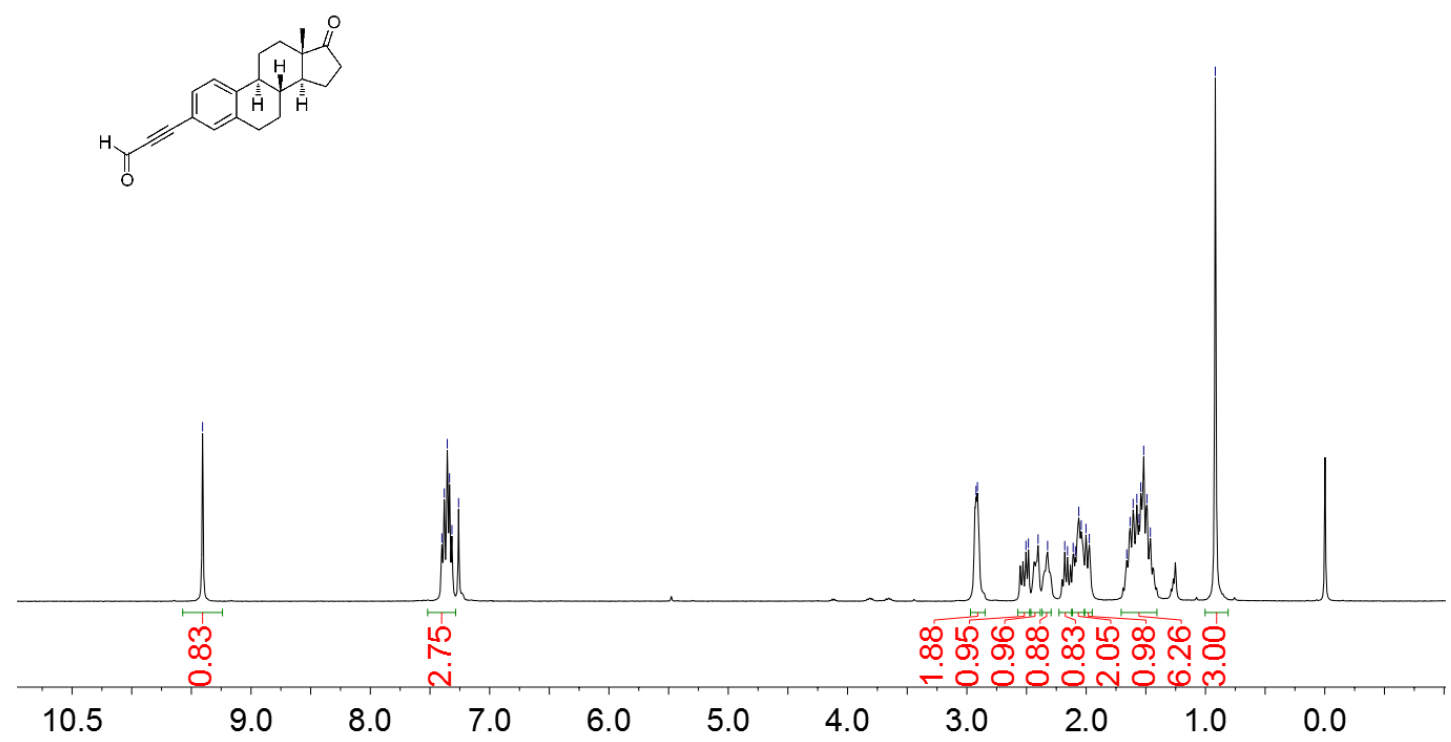

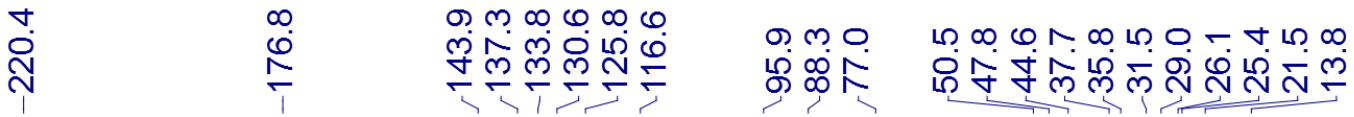

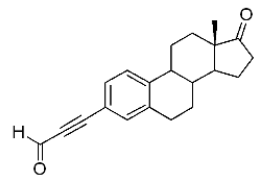

200

170

140

110

80

60

$40 \quad 20$

0 
${ }^{1} \mathrm{H}$ and ${ }^{13} \mathrm{C}$ of compound 15 in $\mathrm{CDCl}_{3}$

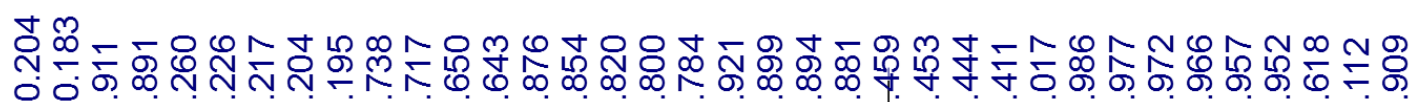
읏 N N N

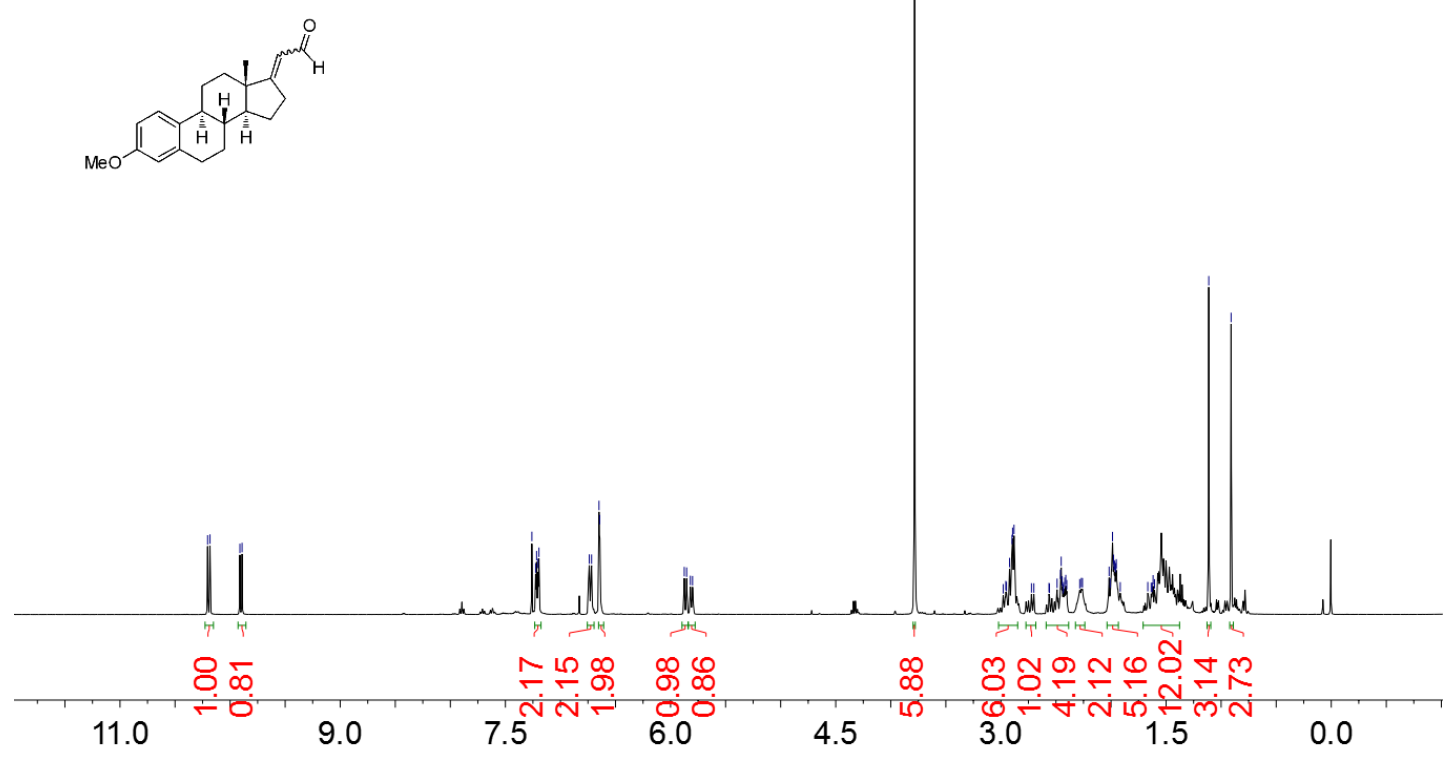

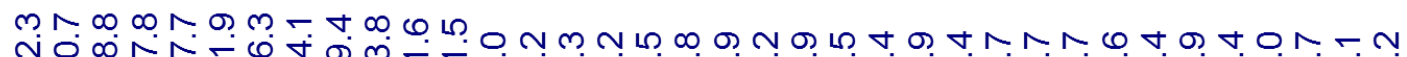

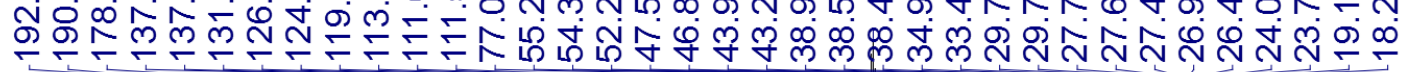
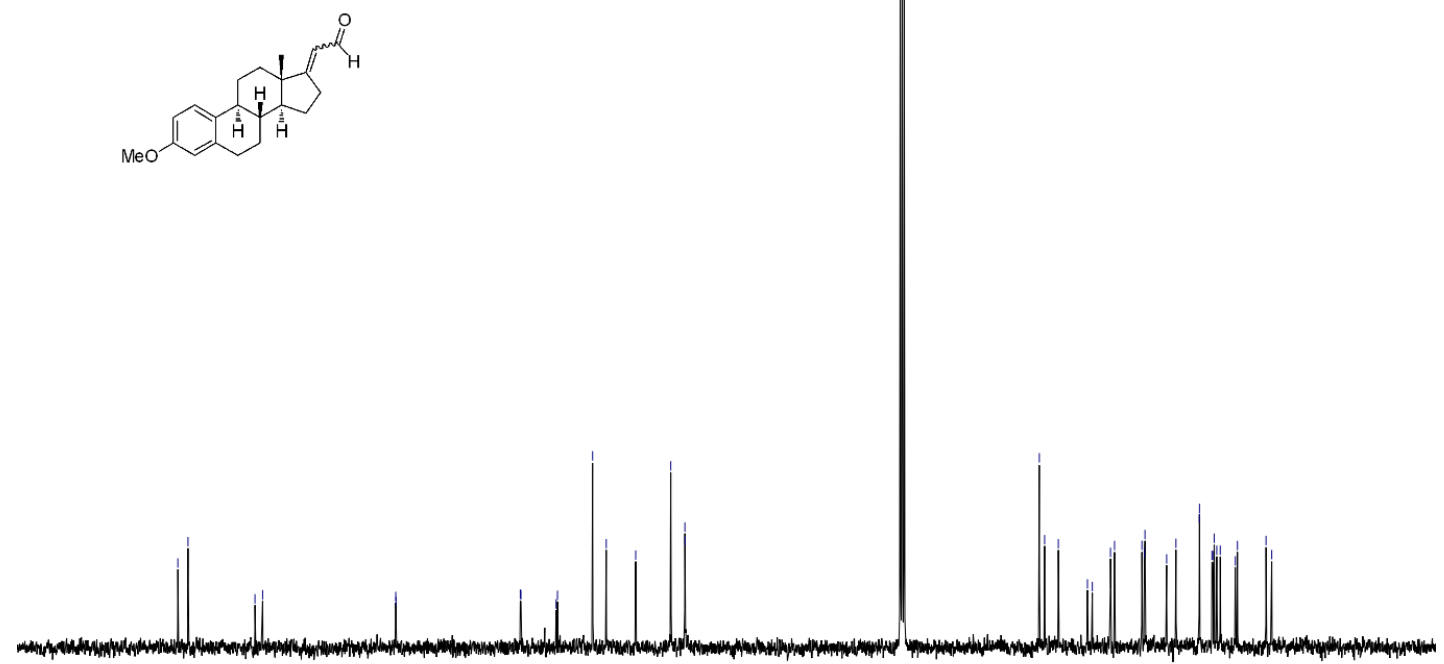

200

180

160

140

120

100

80

60

40

20 